\title{
Design and Analysis of Earthing System for Wind Turbine Generators from Lightning Discharge Currents
}

\author{
by \\ Raghavender Goud Deshagoni
}

\author{
A thesis \\ submitted to the Victoria University of Wellington \\ in fulfilment of the \\ requirements for the degree of \\ Doctor of Philosophy \\ in Engineering.
}

Victoria University of Wellington

2020 



\begin{abstract}
Currently, wind power production is undergoing rapid growth due to the escalating interest in green energy generation. As a result, generators are now choosing to locate wind turbine generators (WTGs) in areas where there is more lightning activity, and earthing problems can be exacerbated further by the soil resistivity being higher than where turbines are usually located. In addition, the desire to capture more energy from the wind has given way to larger WTGs, further increasing the probability of lightning strikes to the structure. This heightened regularity has emphasized the need for an effective grounding system, capable of dissipating the large currents discharged by the lightning into the lightning protection system. This "effective grounding system" must offer a low impedance by limiting the ground potential rise, which is critical due to the wider frequency content of the lightning discharge currents (ranging from DC to several $\mathrm{MHz})$.

The design of an effective grounding system for WTGs depends on the calculation of the minimum length of the earth electrodes, soil resistivity and its frequency-dependency, and the impact of WTG foundation. The calculation of the length of earth electrodes needs an accurate measurement of soil resistivity and modeling of the measured resistivity. Hence, this research considers the measured soil resistivity values of an Australian wind farm and presents an analysis of the soil stratification to identify the optimum soil models. The influence of the soil layers on the WTG grounding system is also investigated to install the earth electrodes. As the resistivity of the soil is frequency-dependent, an analysis is performed to evaluate the effect of the frequency-dependent soil pa-
\end{abstract}


rameters on the WTG grounding system at various frequencies of lightning discharge current. In addition, the impact of the rebar of the WTG foundation on the grounding system is evaluated as the rebar shares the lightning discharge currents. The effective length of the earth electrodes is frequency-dependent, and rebar determines the impedance of the grounding system at high-frequencies. The next step in the grounding design is the design of earth electrodes.

The current dissipating capacity of the earth electrodes depends on soil resistivity, dimensions of the earth electrodes, and burial depth of the electrodes. However, the traditional practice of designing earth electrodes is based on the soil resistivity alone, considering the uniform soil resistivity model. The conventional method of designing earth electrodes based on the uniform soil resistivity is not practical due to non-homogeneous behavior of the soil resistivity. To enhance the WTG earthing system design, this research proposes a novel method to calculate the minimum length of an earth electrode for uniform and two-layer based soil models considering electrode dimensions and burial depth. The grounding impedance achieved when electrode lengths are calculated using the proposed method is compared to grounding impedance values computed using the conventional method. This comparison shows that the proposed method is an improvement on the current convention. In particular, the proposed method gives a grounding impedance value of less than $10 \Omega$ at low frequencies for all soil resistivity values. This results in a reduction in the potential rise of up to $64 \%$ compared to the peak potential value in the conventional method. The benefits offered by the proposed method mean that it can be employed to calculate electrode lengths for the required resistance values based on soil resistivity, electrode dimensions, and burial depth. Such a design may serve as a starting point for an engineer wishing to design a WTG earthing system.

Another challenge noted is the practice of assessing the effectiveness of the WTG grounding system. The conventional method is based on 
achieving a low-frequency resistance of $10 \Omega$ according to the standard IEC 61400-24 and the performance of the grounding system at high frequencies is not considered. Hence, identification of the high-frequency components of the relevant lightning discharge currents is important to understand the performance of the grounding system. An analysis of the wind turbine earthing system for different lightning discharge current wave shapes is performed considering the lightning current waveforms and parameters mentioned in the IEC 61400-24 standard and evaluated the various frequency components and their influence on the WTG grounding system. It is identified that the impedance of the grounding system is minimum for the first short positive stroke current parameters for all the soil resistivity values compared to the first short negative and the subsequent short current wave shapes, although the peak current magnitude is highest for this wave shape. From the analysis of WTG grounding system based on various parameters, this research presents a procedure for assessing the effectiveness of WTG lightning protection system with a focus on the grounding system. It is identified that the effectiveness of the grounding system can be improved by proper design of earth electrodes, optimum soil stratification, and selecting low resistivity soil sites. Finally, various earth electrode configurations are evaluated to identify the better electrode configuration for WTG grounding system.

This thesis provides an in-depth analysis of WTG grounding systems to protect WTGs from lightning strikes. The contributions of this research will help wind farm architects to design effective grounding systems leading to effective lightning protection systems. Finally, the contributions will help to increase the adoption of wind power, resulting in more renewable energy generation. The outcome of this research can be realized to reduce the downtime of WTGs by incorporating the effectiveness of lightning protection system component into the wind farm optimization process. Also, a generalized procedure for calculating the minimum length of earth electrodes for all the soil models can be developed in the future. 


\section{Papers Published From This Thesis}

1. R. D. Goud, T. Auditore, R. Rayudu, and C. P. Moore, “Factors Determining the Effectiveness of a Wind Turbine Generator Lightning Protection System," Accepted for publication in IEEE Transactions on Industry Applications, DOI: 10.1109/TIA.2019.2931866.

2. R. D. Goud, T. Auditore, R. Rayudu, and C. P. Moore, “Frequency domain analysis of a wind turbine generator earthing system for lightning discharge currents," IEEE Access, vol. 7, pp. 60501-60512, 2019.

3. R. D. Goud, T. Auditore, R. Rayudu, and C. P. Moore, "Analysis of earth electrodes of wind turbine generator grounding system under lightning discharge currents," in 2018 IEEE PES Asia-Pacific Power and Energy Engineering Conference (APPEEC), Oct. 2018, pp. 502-507.

4. R. D. Goud, R. Rayudu, C. P. Moore, and T. Auditore, "A Proposed Method for Calculating Earth Electrode Length for a Wind Turbine Generator Grounding System," in 2018 International Conference and Utility Exhibition on Green Energy for Sustainable Development (ICUE), Oct. 2018, pp. 1-6.

5. R. D. Goud, R. Rayudu, C. P. Moore, and T. Auditore, “An evaluation of potential rise in a wind turbine generator earthing system during a 
direct lightning strike," in 2018 International Conference and Utility Exhibition on Green Energy for Sustainable Development (ICUE), Oct. 2018, pp. 1-7.

6. R. D. Goud, R. Rayudu, C. P. Moore, and T. Auditore, "A New Method for Calculating Earth Electrode Length for a Wind Turbine Generator Grounding System based on a Two-Layer Soil Structure," in 2018 International Conference on Power System Technology (POWERCON), Nov. 2018, pp. 1270-1276.

7. R. D. Goud, R. Rayudu, C. P. Moore, and M. Emmanuel, "Analysis of Lightning Generated Over-Voltages on Voltage Regulating Devices in Distribution System with Centralized Wind Turbine Generators," in 2018 Australasian Universities Power Engineering Conference (AUPEC), Nov. 2018, pp.1-6.

8. R. D. Goud, R. Rayudu, C. P. Moore, and D. Burmester, “Lightning protection analysis of main shaft bearings in wind turbine generators," in 2016 IEEE International Conference on Power System Technology (POWERCON), Sep. 2016, pp. 1-6.

9. R. D. Goud, R. Rayudu, Vikash Mantha, and C. P. Moore, "Impact of Short-circuit Ratio on Grid Integration of Wind Farms - A New Zealand Perspective," accepted for publication in 2nd International Conference on Large-Scale Grid Integration of Renewable Energy in India, Sep 2019. 


\section{Acknowledgments}

Foremost, I want to express my deep sense of gratitude and sincere thanks to my supervisors A/Prof Ramesh Rayudu, Dr. Ciaran Moore and Dr. Tony Auditore for their guidance and valuable suggestions. Their wisdom, research insights, and patience allowed me in overcoming the obstacles I have experienced through my research and compile this thesis in present form.

I want to thank my fellow doctoral students of the SPRES research group for their feedback and cooperation. In addition, I would like to thank the administrative and technical staff of the ECS for their support in their respective roles. I would like to thank the Victoria doctoral scholarship and Victoria doctoral submission scholarship for their financial support, which allowed me to dedicate my time in research.

Finally, I would like to say that I am indebted to my parents for everything that they have given to me. I must express my deep appreciation to my wife (Swetha) and son (Vedha Vyas) for their understanding and continuous support throughout this journey. I would like to thank all my other family members and friends for their support. They have always stood by me in everything I have done, providing constant support, encouragement, and love. 


\section{Contents}

1 Introduction 1

1.1 Problem statement $\ldots \ldots \ldots \ldots$. . . . . . . . . 1

1.2 Motivation .................... 2

1.3 Research questions ................. 6

1.4 Research objectives . . . . . . . . . . . . . . 7

1.5 Major contributions . . . . . . . . . . . . . . . . 9

1.6 Thesis Organisation . . . . . . . . . . . . . . . . . 12

2 Literature Review 15

2.1 Basic components of a wind turbine generator . . . . . . 15

2.2 Lightning . . . . . . . . . . . . . . . . . 17

2.3 Incidence of lightning strikes on wind turbines . . . . . . 18

2.4 Lightning current waveforms . . . . . . . . . . . . . 21

2.4.1 Lightning discharge current parameters . . . . . . . 24

2.5 Lightning protection system of wind turbine generators . . . 24

2.5.1 Lightning protection zones . . . . . . . . . . . . 29

2.5.2 Grounding system of wind turbine generators . . . . 31

2.5.3 Earth electrodes . . . . . . . . . . . . . . . . 34

2.6 Soil Resistivity . . . . . . . . . . . . . . . . . . 36

2.7 CDEGS software package $\ldots \ldots \ldots \ldots$

2.8 Chapter summary . . . . . . . . . . . . . . . . . 40 
3 Frequency Domain Analysis of a Wind Turbine Generator Earthing System for Lightning Discharge Currents 41

3.1 Introduction . . . . . . . . . . . . . . . . 42

3.2 Soil Resistivity Measurement and Interpretation . . . . . . . 44

3.2.1 Interpretation of soil resistivity measurements . . . . 45

3.3 Frequency dependent soil parameters . . . . . . . . . . . 48

3.4 Wind Turbine Generator Earthing . . . . . . . . . . . . . . 49

3.5 Lightning Discharge Current . . . . . . . . . . . . 50

3.6 Results and discussion . . . . . . . . . . . . . 51

3.6.1 Soil Models . . . . . . . . . . . . . . . . . 52

3.6.2 WTG earthing impedance variation with soil parameters ...................... 54

3.6.3 Effect of soil layer . . . . . . . . . . . . . 56

3.6.4 Effect of frequency dependent soil parameters . . . . 60

3.6.5 WTG Impedance . . . . . . . . . . . . . . . . . . 63

3.6.6 Ground Potential Rise . . . . . . . . . . . . 70

3.7 Chapter summary . . . . . . . . . . . . . . 76

4 Effects of Direct Lightning Strikes on Wind Turbine Generators 79

4.1 Description of the model . . . . . . . . . . . . . . 80

4.2 Results and Discussion . . . . . . . . . . . . . . . 82

4.2.1 WTG Impedance . . . . . . . . . . . . . . . . . 84

4.2.2 Wind Turbine Earthing System Potential . . . . . . . 87

4.3 Chapter summary . . . . . . . . . . . . . . . . 95

5 Factors Determining the Effectiveness of a Wind Turbine Gener$\begin{array}{ll}\text { ator Lightning Protection System } & 97\end{array}$

5.1 Introduction . . . . . . . . . . . . . . . . . 98

5.2 Assessment framework for WTG lightning protection system 100

5.2.1 Effectiveness of external lightning protection system 100 5.2.1.1 Effectiveness of air-termination system . . . 100

5.2.1.2 Effectiveness of down conduction system . 102 
5.2.2 Effectiveness of internal lightning protection system . 102

5.2.3 Effectiveness of grounding system . . . . . . . . . 103

5.3 Case Study and Simulation Results . . . . . . . . . . . . . . 105

5.3.1 Earth electrode configurations . . . . . . . . . . . 108

5.3.2 Frequency dependent soil parameters . . . . . . . . . 110

5.3.2.1 Effectiveness of the WTG earthing system for various LPLs . . . . . . . . . . . . . . . 110

5.3.2.2 Effectiveness of the WTG earthing system for various electrode configurations . . . . . 118

5.3.2.3 Effectiveness of the WTG earthing system for various soil models . . . . . . . . . . 128

5.4 Chapter summary . . . . . . . . . . . . . . 133

6 A Proposed Method for Calculating Earth Electrode Length for a Wind Turbine Generator Earthing System 135

6.1 Introduction . . . . . . . . . . . . . . . . . . . 135

6.2 Soil Models . . . . . . . . . . . . . . . . . . . . . . 137

6.3 Equivalent soil resistivity model . . . . . . . . . . . . . 138

6.4 Proposed method . . . . . . . . . . . . . . . . . . . . . . . . 140

6.4.1 Proposed Method for Calculating Earth Electrode

Length using a Uniform soil Resistivity Model . . . . 140

6.4.2 Proposed method for calculating the length of the earth electrode for a horizontal two-layer soil resistivity model . . . . . . . . . . . . . . . . 143

6.5 Results and discussion . . . . . . . . . . . . . . . . . 145

6.5.1 Frequency dependency of electrode length . . . . . . 147

6.5.2 Case Study: Three proposed wind sites in Australia . 155

6.5.3 Potential Distribution . . . . . . . . . . . . . 161

6.6 Chapter summary . . . . . . . . . . . . . . 165

7 Conclusions and Future Work 167

7.1 Conclusions . . . . . . . . . . . . . . . . 167 
7.2 Future Work . . . . . . . . . . . . . . . . . . 170 


\section{List of Figures}

1.1 Estimated renewable energy share of global production, end of 2017 [8]. . . . . . . . . . . . . . . . . . 3

1.2 Wind power global capacity and annual additions, 2007$2017[8] \ldots \ldots \ldots$. . . . . . . . . . . . . . . 3

2.1 Wind turbine generator components [39]. . . . . . . . . 16

2.2 Tripole charges in a cloud [28]. . . . . . . . . . . . . . . 18

2.3 The four types of cloud-to-ground lightning flashes as defined from the direction of leader propagation and the charge on the initiating leader [44] . . . . . . . . . . . . . 19

2.4 Waveform of the standard lightning discharge current [63]. . 22

2.5 Maximum values of lightning parameters [17]. . . . . . . . 25

2.6 Components of a lightning protection system [90]. . . . . . . 27

2.7 Application of rolling sphere method to a wind turbine [100]. 28

2.8 Lightning protection zones in a wind turbine [100]. . . . . . 32

2.9 Perspective view of wind turbine foundation (not to scale). . 34

2.10 Minimum length $\left(l_{1}\right)$ of earth electrode according to the class of lightning protection level [17]. . . . . . . . . . . 35

2.11 Work flow diagram of CDEGS software package. . . . . . . 39

3.1 Soil resistivity measurement using four point Wenner method [152]. . . . . . . . . . . . . . . . . 45 
3.2 Frequency response of the WTG earthing impedance with change in soil resistivity with a constant relative permittivity. 55

3.3 Frequency response of the phase angle with change in soil resistivity with a constant relative permittivity. . . . . . . 55

3.4 Frequency response of the WTG earthing impedance with change in relative permittivity with a constant soil resistivity $10 \mathrm{k} \Omega \mathrm{m} \ldots \ldots \ldots$. . . . . . . . . . . 56

3.5 Frequency response of the phase angle with change in rela-

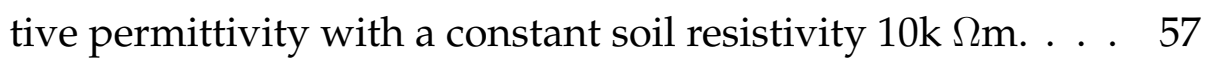

3.6 WTG earthing impedance at $5 \mathrm{kHz}$ frequency with constant resistivity of top layer. . . . . . . . . . . . . 58

3.7 WTG earthing impedance at $5 \mathrm{kHz}$ frequency with constant resistivity of bottom layer. . . . . . . . . . . . . . . . 59

3.8 WTG earthing impedance with frequency dependent soil resistivity for $25 \Omega \mathrm{m} . \ldots \ldots 0$

3.9 WTG earthing impedance with frequency dependent soil

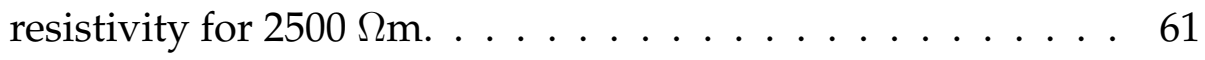

3.10 WTG earthing impedance with frequency dependent soil permittivity. . . . . . . . . . . . 6 62

3.11 WTG earthing impedance with frequency dependent soil parameters for $2500 \Omega \mathrm{m}$. . . . . . . . . . . . . . 63

3.12 WTG earthing impedance of WTG-1-T1 with and without Rebar connected to earthing system for frequency independent soil parameters (Uniform soil model). . . . . . . . . . . 64

3.13 WTG earthing impedance of WTG-1-T1 with and without Rebar connected to earthing system for frequency independent soil parameters (Two-Layer soil model). . . . . . . . . 65

3.14 WTG earthing impedance of WTG-1-T1 with and without Rebar connected to earthing system for frequency independent soil parameters (Three-Layer soil model). . . . . . . . . 65 
3.15 WTG earthing impedance of WTG-1-T1 with ring electrode for frequency independent soil parameters. . . . . . . . . . 66

3.16 Phase angle of WTG-1-T1 with ring electrode for frequency independent soil parameters. . . . . . . . . . . . . 67

3.17 Comparison of WTG earthing impedance of WTG-1-T1 with ring electrode for frequency independent and dependent soil parameters. . . . . . . . . . . . . . . . 68

3.18 Comparison of WTG earthing impedance of WTG-1-T1 with ring electrode and ring \& horizontal electrodes for frequency independent soil parameters. . . . . . . . . . . . . . . 69

3.19 Comparison of WTG earthing impedance of WTG-1-T1 with ring \& horizontal electrodes for frequency independent and dependent soil parameters. . . . . . . . . . . . . 70

3.20 Ground potential rise for WTG-1-T1 with three-layer soil resistivity with electrodes at $5 \mathrm{kHz}$. . . . . . . . . . . 71

3.21 Ground potential rise for WTG-1-T1 with three-layer soil resistivity with ring electrodes at $5 \mathrm{kHz} \ldots \ldots . \ldots 72$

3.22 Ground potential rise for WTG-1-T1 with three-layer soil resistivity with ring and horizontal electrodes at $5 \mathrm{kHz}$. . . . 73

3.23 Ground potential rise for WTG-1-T1 with three-layer soil resistivity with ring and horizontal electrodes at $1 \mathrm{MHz}$. . . . . 74

3.24 Ground potential rise for WTG-1-T1 with three-layer soil resistivity with ring and horizontal electrodes at $1 \mathrm{MHz}$. . . . 75

4.1 WTG earthing system. . . . . . . . . . . . . . . . . 80

4.2 Lightning current waveform of the first short positive stroke. 81

4.3 Lightning current waveform of the first short negative stroke. 81

4.4 Lightning current waveform of a subsequent short stroke. . 82

4.5 WTG earthing impedance for the first short positive stroke lightning current parameters. . . . . . . . . . . . . 84

4.6 WTG earthing impedance for the first short negative stroke lightning current parameters. . . . . . . . . . . . 86 
4.7 WTG earthing impedance for a subsequent short stroke lightning current parameters. . . . . . . . . . . 87

4.8 Scalar potential at $10.8 \mathrm{kHz}$ for the first short positive lightning stroke on WTG-1. . . . . . . . . . . . . . 88

4.9 Scalar potential at $410 \mathrm{kHz}$ for the first short positive lightning stroke on WTG-1. . . . . . . . . . . . . . . . 89

4.10 Scalar potential at $5 \mathrm{kHz}$ for the first short negative lightning stroke on WTG-1. . . . . . . . . . . . . . 90

4.11 Scalar potential at $1 \mathrm{MHz}$ for the first short negative lightning stroke on WTG-1. . . . . . . . . . . . . . . . 91

4.12 Scalar potential at $5.33 \mathrm{kHz}$ for a subsequent short lightning stroke on WTG-1. . . . . . . . . . . . . . . 92

4.13 Scalar potential at $1 \mathrm{MHz}$ for a subsequent short lightning stroke on WTG-1. . . . . . . . . . . . . . . . . . . 93

4.14 Scalar potential at $5.46 \mathrm{MHz}$ for a subsequent short lightning stroke on WTG-1. . . . . . . . . . . . . . . 94

5.1 Factors determining the effectiveness of a WTG LPS. . . . . . 101

5.2 Step and touch voltages [190]. . . . . . . . . . . . . . 104

5.3 Representation of frequencies of lightning discharge current. 108

5.4 Perspective view of WTG commonly used earthing electrode structures (not to scale). . . . . . . . . . . . . . 109

5.5 Perspective view of WTG earthing system with eight cross electrodes (not to scale). . . . . . . . . . . . . . . . . 109

5.6 Perspective view of WTG earthing system with grid electrodes (not to scale). . . . . . . . . . . . . . . 110

5.7 WTG grounding resistance for different lightning protection levels. . . . . . . . . . . . . . . . . . . . . . . . 112

5.8 Impulse coefficient of the WTG grounding system for first short negative lightning discharge current parameters. . . . 113

5.9 Potential distribution of the WTG grounding system at $5 \mathrm{kHz}$ for soil resistivity of $100 \Omega \mathrm{m}, \mathrm{LPL}-1 \ldots$. . . . . . . . . . 114 
5.10 Potential distribution of the WTG grounding system at $1 \mathrm{MHz}$ for soil resistivity of $100 \Omega \mathrm{m}, \mathrm{LPL}-1 . \ldots \ldots$

5.11 Peak potential magnitude at various lightning protection levels. . . . . . . . . . . . . . . . . . . 116

5.12 Step voltage for various lightning protection levels. . . . . . 117

5.13 Impedance and phase angle of the WTG earthing system for $\rho=100 \Omega$ m. . . . . . . . . . . . . . . . 120

5.14 Impedance and phase angle of the WTG earthing system

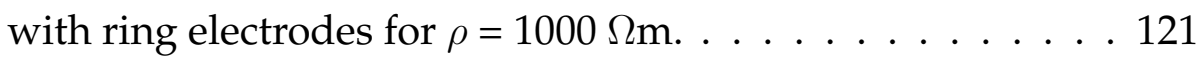

5.15 Impedance of the WTG earthing system with different electrodes for $\rho=1000 \Omega$ m. . . . . . . . . . . . . . . . 122

5.16 Impedance and phase angle of the WTG earthing system with five ring electrodes for $\rho=2000 \Omega \mathrm{m} . \ldots \ldots$. . . . . 123

5.17 Impedance of the WTG earthing system for different electrodes for $\rho=2000 \Omega$ m. . . . . . . . . . . . . . . . . . . 124

5.18 Impedance of the WTG earthing system for $\rho=2000 \Omega \mathrm{m}$ for various electrode configurations. . . . . . . . . . . 125

5.19 Impedance and phase angle of the WTG earthing system with vertical electrodes for $\rho=10 \mathrm{k} \Omega \mathrm{m}$. . . . . . . . . . 126

5.20 Impedance of the WTG earthing system with different electrodes for $\rho=10 \mathrm{k} \Omega \mathrm{m}$. . . . . . . . . . . . . . . 127

5.21 Comparison of low frequency resistance for various electrode configurations for uniform soil resistivities. . . . . . . . 128

5.22 Comparison of impulse coefficient for various electrode configurations for various uniform soil resistivities. . . . . . 129

5.23 Comparison of low-frequency resistance for various electrode configurations with various soil models . . . . . . . . . 130

5.24 Comparison of impulse coefficient for various electrode configurations with various soil models and frequency dependent soil parameters. . . . . . . . . . . . . . . . . . . 130 
5.25 Peak potenial for various electrode configurations and soil models. . . . . . . . . . . . . . . . . . . 131

5.26 Step voltage for various electrode configurations and soil models. . . . . . . . . . . . . . . . . . 132

6.1 Uniform soil model [152]. . . . . . . . . . . . . . . . . . . 138

6.2 Horizontal two-layer soil model [152] . . . . . . . . . . . . . 139

6.3 Horizontal multi-layer soil model [198]. . . . . . . . . . . . . 139

6.4 Coordinates for buried ring of wire [146] . . . . . . . . . . . . 141

6.5 Proposed method for calculating earth electrode length for two-layer soil structure. . . . . . . . . . . . . . . . . 144

6.6 Frequency dependent soil resistivity evaluated based on the frequency-dependent soil parameters equations. . . . . . . . 147

6.7 Frequency dependent WTG earth electrode length according to IEC 61400-24. . . . . . . . . . . . . . . . 149

6.8 WTG earth electrode length for $10 \Omega$ resistance for $0.5 \mathrm{~m}$ buried depth with the proposed method. . . . . . . . . . 150

6.9 WTG earth electrode length for $10 \Omega$ resistance for $2 \mathrm{~m}$ buried depth with the proposed method. . . . . . . . . . 152

6.10 WTG earth electrode length for $5 \Omega$ resistance for $0.5 \mathrm{~m}$ buried depth with the proposed method. . . . . . . . . . 153

6.11 WTG earth electrode length for $5 \Omega$ resistance for $2 \mathrm{~m}$ buried depth with the proposed method. . . . . . . . . . . . . . 154

6.12 Potential distribution for the electrode design based on uniform soil resistivity. . . . . . . . . . . . . . . 161

6.13 Potential distribution for the electrode design based on top layer soil resistivity of the two-layer soil model. . . . . . . 162

6.14 Potential distribution for the electrode design based on bottom layer soil resistivity of the two-layer soil model. . . . . . 163

6.15 Potential distribution for the electrode design based on the proposed method. . . . . . . . . . . . . . . . . 164 


\section{List of Tables}

2.1 Lightning protection zones [63]. . . . . . . . . . . . . 30

2.2 Soil resistivity values of typical soils and water at $25^{\circ} \mathrm{C}$ [151] 37

3.1 Soil Resistivity Measurements . . . . . . . . . . . . . . 46

3.2 Soil Structure of WTG-1 for Different Soil Models . . . . . 52

3.3 Percentage Error of Soil Models . . . . . . . . . . . . . 53

4.1 Soil Resistivity Values of Wind Turbine Locations _. . . . 82

4.2 Dominant Frequencies and Corresponding Amplitude for Different Lightning Strokes . . . . . . . . . . . . . 85

5.1 Effectiveness of air-termination system . . . . . . . . . 105

5.2 Effectiveness of down conduction system . . . . . . . . 106

5.3 Frequency Dependent Soil Resistivity . . . . . . . . . . . 111

5.4 Frequency Dependent Relative Permittivity . . . . . . . . . . 111

6.1 Mean radius of earth electrode $r_{e}(\mathrm{~m}) . \ldots \ldots . \ldots 146$

6.2 Simulation scenarios . . . . . . . . . . . . . . . . 156

6.3 Soil Resistivity Values . . . . . . . . . . . . . . . . 157

6.4 Required electrode length $(\mathrm{m})$ to ensure resistance below $10 \Omega$, calculated using a variety of soil resistivity models and for various WTG locations . . . . . . . . . . . . . 159

6.5 WTG Earthing Impedance . . . . . . . . . . . . . . . 160 


\title{
Nomenclature
}

\author{
$A C \quad$ Alternating Current \\ CDEGS Current Distribution, Electromagnetic fields, Grounding, \\ and Soil structure analysis \\ $C G \quad$ Cloud to Ground \\ DC Direct Current \\ DFIG Doubly-Fed Induction Generator \\ EMI Electromagnetic Interference \\ FDTD Finite Difference Time Domain \\ FEMTD Finite Element Method in Time Domain \\ GFRP Glass Fibre Reinforced Plastic \\ GPR Ground Potential Rise \\ GW Giga Watt \\ IEC International Electrotechnical Commission \\ IEEE Institute of Electrical and Electronics Engineers \\ $k A \quad$ Kilo Ampere \\ $k H z \quad$ Kilo Hertz
}




$\begin{array}{cl}k V & \text { Kilo Volt } \\ L E M P & \text { Lightning Electromagnetic Pulses } \\ L P L & \text { Lightning Protection Level } \\ L P M S & \text { Lightning Protection Measures } \\ L P S & \text { Lightning Protection System } \\ L P Z & \text { Lightning Protection Zones } \\ M H z & \text { Mega Hertz } \\ M o M & \text { Method of Moment } \\ M W & \text { Mega Watt } \\ N E A & \text { Numerical Electromagnetic Analysis } \\ P E E C & \text { Partial Element Equivalent Circuit } \\ S P D & \text { Surge Protective Device } \\ T L M & \text { Transmission Line Matrix } \\ U P S & \text { Uninterruptible Power Supply } \\ & \text { Wind Turbine Generator } \\ & \\ & \\ & \end{array}$




\section{Chapter 1}

\section{Introduction}

\subsection{Problem statement}

Amongst all renewable energy sources, power generation from wind energy conversion systems is growing rapidly. This is partly due to the fact that wind energy has been recognized as an environmentally friendly and economically competitive means of electric power generation [1]. Furthermore, wind energy has expanded its base from high to low wind speed sites and from small scale kilowatt generation to commercial level megawatt class systems [1]. The increase in power generation capacity of wind turbine generators (WTGs) is due to improvements in technology and the increase sizes of the WTGs.

One disadvantage of wind turbine generators is that they are extremely vulnerable to lightning strikes due to their height, size, shape, and location [2], [3]. They are typically up to $120 \mathrm{~m}$ high with rotors that span $80 \mathrm{~m}$ in diameter, thus increasing their exposure and risk to direct lightning strikes [4]. They are also often placed in isolated mountainous regions that coincide with areas of considerable thunderstorm activity, which increases the risk of lightning strike [5]. Alternative locations are on level plains where WTG are predominantly high profile structures. Moreover, WTGs are often installed at high soil resistivity sites. This means that lightning 
strikes can damage not just individual wind turbines but also sections of a wind farm and parts of the grid [6]. The future growth of wind energy may become constrained if improvements in lightning protection systems are not realised.

Given the risk to the growth of wind energy due to lightning strikes [7], this thesis investigates potential improvements to lightning protection systems for WTGs. It is important to dissipate the lightning discharge current to the local earth by providing a low impedance path. Amongst all the components of a lightning protection system, the enhancement of the earthing system design is addressed in this thesis.

\subsection{Motivation}

In 2017 the proportion of net additions to global power generating capacity that came from renewable energy sources was estimated to be in excess of $70 \%$, with some countries realising much higher contributions compared to the global average [8]. By the end of 2017, renewables were estimated to supply $26.5 \%$ of global electricity, as shown in Fig. 1.1 [8]. Hydropower contributes the most of all renewable sources with a share of $16.4 \%$ to total energy sources, followed by wind power with a significant 5.6\% share. Moreover, the annual addition of wind power has been increasing since 2007, from $1000 \mathrm{GW}$ total global installed capacity to 2195 GW in 2017, as illustrated in Fig. 1.2 [8].

Technological advances, expansion into new markets with enhanced resources, and improved financing conditions have reduced capital and operational costs, particularly for wind energy [8]. Onshore wind power is cost-competitive with new fossil capacity, even without accounting for externalities. Wind power was also the most cost-effective option for new grid-based power in 2015 in many markets, including Canada, Mexico, New Zealand, South Africa, Turkey, and parts of Australia, China and the United States [8]. 


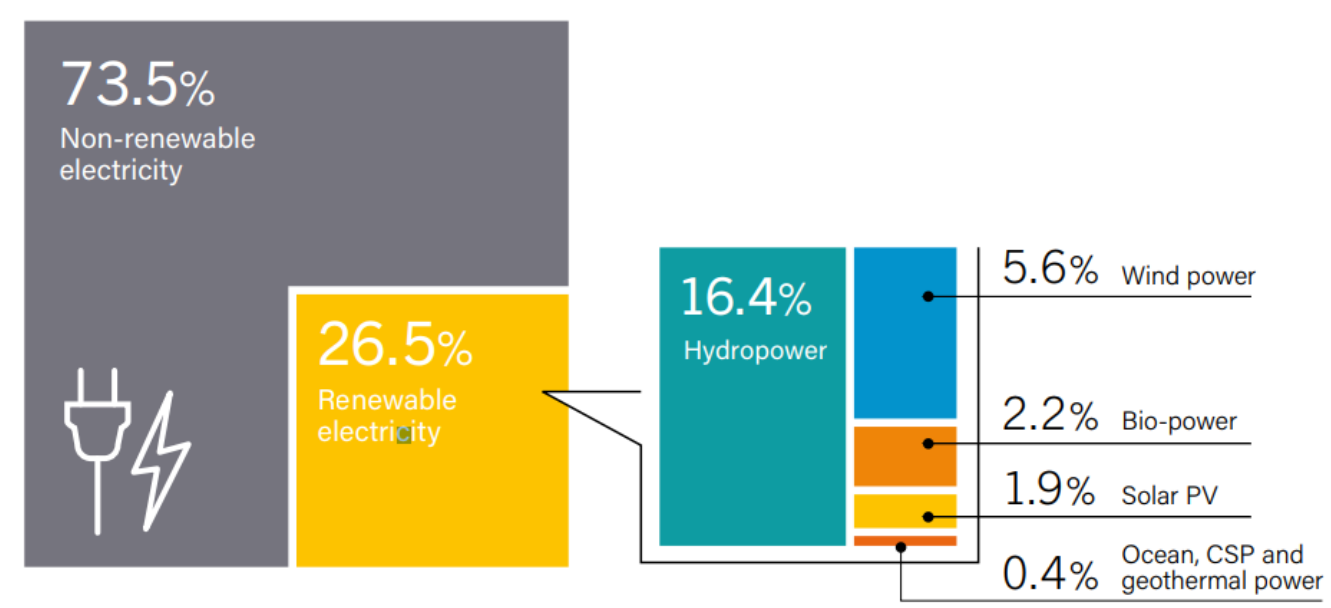

Figure 1.1: Estimated renewable energy share of global production, end of 2017 [8].

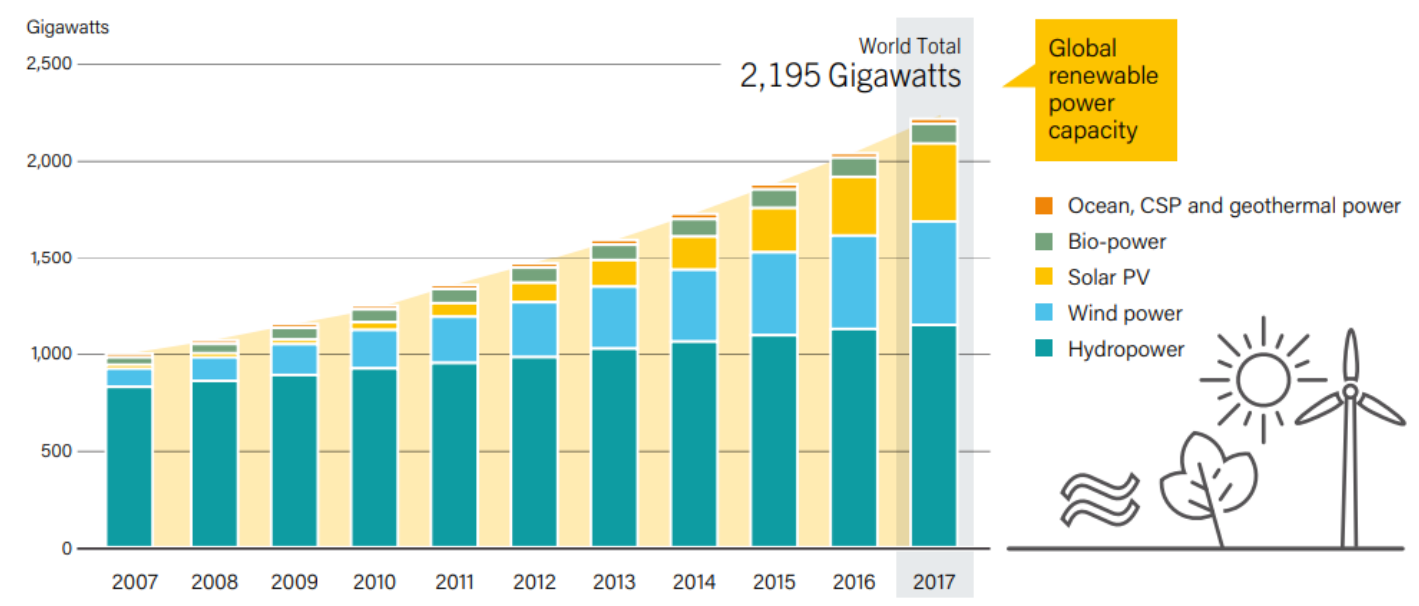

Figure 1.2: Wind power global capacity and annual additions, 20072017 [8]. 
The reduced costs for wind energy are due to a surge in energy generation from individual WTGs as a result of their expanding size and escalated installations at high altitudes [9]. As a result, the chances of lightning strikes on wind turbines have increased, motivating renewed study into the design of lightning protection system (LPS) [10], [11].

In the event of a lightning strike on a wind turbine generator, the transient lighting discharge currents dissipate into the ground through the LPS installed in the WTGs [12], [13]. The LPS consists of an air termination system [14], which protects the WTG from direct lighting strikes, a down conduction system providing a low impedance path to the earthing system, and an earthing system to dissipate the lightning discharge currents into the general mass of the earth [15].

The lightning discharge currents may induce over-voltages on the electrical and electronic equipment of the WTG [16] in the absence of an effective earthing system being installed. There are different types of earthing systems available in the literature, however, a relevant international standard IEC 61400-24 [17] recommends designing a type B earthing system for wind turbine lightning protection, which features a ring electrode buried around the perimeter of the WTG along with additional radial or vertical electrodes if required [18], [19].

The IEC 61400-24 standard [17] recommends an earthing resistance of less than $10 \Omega$ for stand-alone WTG earthing systems. Moreover, the standard provides guidelines for selecting the length of earth electrodes to achieve this low resistance [20], [21]. However, it is challenging to provide such a low resistance earthing system for the wind turbines installed at high soil resistivity sites.

Wind turbines are often located in regions with high keraunic levels [22], [23] and high soil resistivity [24], [25] where the wind availability is sufficient to produce power. The keraunic level is the number of thunderstorm days per year. High soil resistivity increases the grounding resistance, which in turn decreases the effectiveness of the lightning protection 
systems. Due to their height, shape, and continuous rotation, WTGs are extremely vulnerable to lightning strikes [26]. Modern wind turbines with structures in excess of $100 \mathrm{~m}$ will invariably experience both upward and downward lightning strikes [27], [28]. In addition to the damages to the blades of WTG caused by lightning strikes, the swept stroke phenomenon, where arc channel of the lightning strike remain attached to the blade for the duration of the lightning strike [29], can lead to the erosion and tracking on the blades due to upward lightning strikes [28].

Lightning discharge currents comprise high frequency components [30], [31] which experience higher impedance in the earthing system compared to low-frequency components, leading to higher potential rise. Hence, the performance of the WTG is adversely affected by the combined influence of high lightning strike incidence, large magnitude lightning discharge current and high soil resistivity [24].

A lightning strike to an unprotected WTG can damage the blades, destroy the mechanical parts and disrupt the electrical and control systems [27]. Furthermore, people in and around WTGs are exposed to hazards from the step and touch voltages or explosions and fires caused by a lightning flash [17]. When a lightning strikes the WTG, due to the ground potential rise, a human or animal that touches an exposed metallic part can experience a touch potential, and the potential difference between the two legs will creates a step potential [32]. There have been reports in the literature that at least $50 \%$ of WTGs damaged by lightning strikes have dielectric breakdown in their LV network and the electrical components inside the WTG [17].

Moreover, there is no clear guidance in the IEC 61400-24 standard [17] regarding the length of the earthing electrodes beyond certain values of soil resistivity. The selection of the length of the earth electrodes according to the standard is based on a uniform soil resistivity model. However, the resistivity of soil is inherently inhomogeneous [33]. Hence, there is a need for a method to select the length of the earth electrodes for non-uniform 
soil models. In addition, the dimensions and burial depths of the earth electrodes are not considered when designing the earthing system using traditional methods.

Considering the above, there is an opportunity to enhance the design of earthing systems for an effective lightning protection system. This is achieved by considering all the earth electrode parameters when designing an earthing system.

\subsection{Research questions}

The overall goal of this thesis is to analyse the earthing systems of WTGs to protect them form lightning discharge currents and thereby improve the design of earthing systems. This research will help answer the following research questions:

1. What is the role of soil stratification in the design of WTG earthing system for lightning discharge currents?

This research question explores the following sub-questions in this research:

(a) What is the impact of soil stratification on the design of an earthing system?

(b) How should soil resistivity models be evaluated and chosen for WTG earthing system design calculations?

(c) What is the effect of soil modelling on the selection of earth electrodes?

2. What are the parameters required to assess the effectiveness of the WTG LPS?

This research question will address the following points:

(a) What are the advantages of evaluating the effectiveness of an LPS of a WTG? 
(b) What is the current practice of assessing the effectiveness of a WTG LPS?

(c) Do the electrodes of an earthing system have any significance in the effectiveness of the LPS of WTG?

(d) What electrode configuration gives the best performance of an earthing system of a WTG for lightning discharge current?

(e) What is the effect of designing an earthing system to various lightning protection levels?

(f) What frequencies does a lightning discharge current contain and what is the effect of frequencies on the earthing system?

(g) What is the impact of integrating the rebar of the WTG foundation into the earthing system?

3. What are the shortfalls of the current practice of designing the WTG earthing system?

This research question investigates the following sub-questions:

(a) What are the parameters to be considered while designing an earthing system for a WTG?

(b) Does the current practice of designing the earthing system of a WTG consider all the influential parameters?

(c) Can the design of WTG earthing system be improved? If so, how?

\subsection{Research objectives}

This thesis aims to improve the lightning protection system of a wind turbine generator by enhancing the earthing system design. This is to be achieved by evaluating suitability of soil resistivity models, factors influencing soil parameters and an enhanced method to design earth electrodes. 
In order to accomplish the overall goal and provide answers to research questions, a set of research objectives have been established as a research guide. They include the following:

\section{Objective 1:}

Evaluation of various soil stratification models and their effect on the impedance of a wind turbine generator earthing system is important in designing an effective earthing system. Also, the effect of frequency dependent soil parameters, effect of burial earth electrodes in different soil layers and the effect of rebar on the WTG earthing impedance are significant. This thesis performs an analysis of WTG earthing impedance for various soil structures and explores the influence of different soil parameters. The simulations and analysis are carried out using measured soil resistivity values. This objective addresses research question-1(a), (b), (c) and 2(g).

\section{Objective 2:}

Soil resistivity is one of the major components that decides the WTG earthing impedance, hence earthing electrodes are typically designed based on traditional methods that consider only the resistivity of the soil. However, other factors, such as electrode dimensions and burial depth, are important in calculating the minimum length of earth electrodes. Moreover, the earthing system for a horizontal multi-layer soil structure is more practical than a uniform soil stratification. This thesis aims to propose a method to calculate the earth electrode length considering a number of relevant parameters, not just the resistivity of the soil. This objective addresses research question-3(a),(b) and (c).

\section{Objective 3:}

The earthing electrodes in combination with the rebar structure of the WTG foundation determine the earthing impedance of a wind turbine grounding system. Hence, it is important to choose a suitable 
configuration and dimensions for these electrodes. This study computes the earthing impedance and ground potential rise of a WTG earthing system for various earthing electrodes for uniform and horizontal multi-layer soil structures to achieve a low impedance earthing system. This objective addresses research question-2(d).

\section{Objective 4:}

The design of a lightning protection system is based on lightning protection level parameters. This research analyses various lightning discharge current parameters and lightning protection level parameters to assess the effect on the earthing system. This objective addresses research question-2(e) and (f).

\section{Objective 5:}

The current practice of designing an earthing system aims to reduce the low-frequency resistance. However, the high-frequency components of lightning discharge currents leads to high impedance of the earthing system. Hence, various characteristics of the WTG earthing system to design an effective lightning protection system are identified in this research. The assessment of the lightning protection system at the design stage of a wind farm reduces future down time, leading to increased annual energy yield from the wind farms. This research provides a framework to assess the effectiveness of a wind turbine lightning protection system. This objective addresses research question-2(a),(b),(c) and (d).

\subsection{Major contributions}

This thesis contains the following major contributions.

1. The conventional design of a wind turbine earthing system is based on a uniform soil model with frequency independent soil parameters. This thesis, through modelling and analysis of WTG earthing 
impedance for various soil structures, will describe a method for selecting the optimum soil model for the earthing design for a given WTG site. Also, by studying the effect of frequency dependent soil parameters on the soil, an analysis of WTG earthing impedance is performed. This thesis propose a procedure to determine the optimum location for an earth electrode in a horizontal multi-layer soil structure. The findings of this research have been peer-reviewed and published in:

R. D. Goud, T. Auditore, R. Rayudu, and C. P. Moore, "Frequency domain analysis of a wind turbine generator earthing system for lightning discharge currents," IEEE Access, vol. 7, pp. 60501-60512, 2019.

2. A framework to assess the effectiveness of the WTG lightning protection system is proposed in this research. The framework will help the wind farm designers to site the individual wind turbines in such a way that reduces the number of lightning strikes on a WTG and also improves the effectiveness of the grounding system by proper selection of the grounding electrodes. The findings of this research have been peer-reviewed and published in:

Publication is submitted to IEEE Transactions on Industry Applications and is accepted for publication:

R. D. Goud, T. Auditore, R. Rayudu, and C. P. Moore, “Factors Determining the Effectiveness of a Wind Turbine Generator Lightning Protection System," accepted for publication in IEEE Transactions on Industry Applications, DOI: 10.1109/TIA.2019.2931866.

3. A new method is proposed to calculate the minimum required length of earth electrode by considering soil resistivity of the proposed site, electrode dimensions, and electrode burying depth. Also, a method is proposed to calculate the electrode length for a horizontal two-layer soil model. The calculated earthing impedances are 
compared with the traditional method by carrying out a frequency response of the WTG earthing impedance. The findings of this research have been peer-reviewed and published in:

R. D. Goud, R. Rayudu, C. P. Moore, and T. Auditore, "A Proposed Method for Calculating Earth Electrode Length for a Wind Turbine Generator Grounding System," in 2018 International Conference and Utility Exhibition on Green Energy for Sustainable Development (ICUE), Oct 2018, pp. 1-6.

R. D. Goud, R. Rayudu, C. P. Moore, and T. Auditore, "A New Method for Calculating Earth Electrode Length for a Wind Turbine Generator Grounding System based on a Two-Layer Soil Structure," in 2018 International Conference on Power System Technology (POWERCON), Nov 2018, pp. 1270-1276.

4. The analysis of designing a grounding system to various lightning discharge current and lightning protection level parameters provides a guidance to select the appropriate design parameters. The findings of this research have been peer-reviewed and published in:

R. D. Goud, R. Rayudu, C. P. Moore, and T. Auditore, "An evaluation of potential rise in a wind turbine generator earthing system during a direct lightning strike," in 2018 International Conference and Utility Exhibition on Green Energy for Sustainable Development (ICUE), Oct 2018, pp. 1-7.

5. Finally, through modelling and analysis of various earth electrodes for a WTG earthing system based on uniform and horizontal soil models, this thesis proposes an optimum electrode configuration to achieve a low impedance for the wind turbine earthing impedance. WTG earthing impedances for various lightning discharge currents and associated high frequencies are compared and recommendations are made for an improved earthing system. The findings of 
this research have been peer-reviewed and published in:

R. D. Goud, T. Auditore, R. Rayudu, and C. P. Moore, "Analysis of earth electrodes of wind turbine generator grounding system under lightning discharge currents," in 2018 IEEE PES Asia-Pacific Power and Energy Engineering Conference (APPEEC), Oct 2018, pp. 502-507.

\subsection{Thesis Organisation}

The remainder of this thesis is organized as follows:

Chapter 2 starts with an overview of the various components of a wind turbine generator. It then provides an introduction to the lightning phenomenon and the incidence of lightning strikes to a wind turbine. A discussion on lightning current waveforms and various lightning discharge current parameters is presented. Emphasis is placed on the lightning protection system and lightning protection zones of a wind turbine. In addition, this chapter presents an introduction to the wind turbine grounding systems and associated components required for designing a grounding system. The last part of this chapter discusses soil resistivity and typical resistivity values of the soil.

In Chapter 3, three locations at an Australian wind farm were modelled based on measured soil resistivity data. Four soil resistivity models were considered: uniform, multi-layer horizontal, vertical, and exponential variation with depth. Full-wave electromagnetic simulations were performed at different lightning discharge current frequencies to determine the expected ground potential rise and WTG earthing impedance in the event of a lightning strike. The effect of frequency-dependent soil parameters on the WTG earthing system are also analysed, along with the impact of foundation rebar on the grounding impedance. The research output presented in this chapter has resulted in the publication of [34].

Chapter 4 presents an analysis of the wind turbine earthing system 
for a horizontal multi-layer soil model in the event of a direct lightning strike on the wind turbine generator for various lightning discharge current waveforms. The analysis of the frequency components and peak current magnitudes of different lightning current waveforms and their impact on the earthing system of the wind turbine is performed. The results presented in this chapter have resulted in the publication of [35].

In Chapter 5, a framework to assess the effectiveness of the wind turbine lightning protection systems at the wind farm design phase is presented. The research in this chapter shows that the grounding system plays a critical role in the wind turbine lightning protection system. For this reason, an analysis of various influential parameters of a grounding system design is performed. This chapter also presents an analysis of different earthing electrode configurations for the lightning protection of a wind turbine grounding system and identifies the best electrode configuration for WTG earthing system. Besides, this chapter presents an analysis of different earth electrode configurations and lengths for lightning discharge current frequencies for different soil resistivity values based on frequency independent and dependent soil parameters. The research output presented in this chapter have resulted in the publication of [36].

In Chapter 6, a novel method is proposed to calculate the minimum length of an earth electrode for a WTG grounding system by considering the electrode dimensions and burial depth. A method to calculate the minimum length of an earth electrode for a WTG grounding system based on a two-layer soil model is also proposed. This methodology can be used to calculate the electrode length for a required value of grounding resistance. The proposed method is tested for various WTG sites and compared with the traditional practices. The results presented in this chapter have been published in $[37,38]$.

Chapter 7 presents a summary of the key findings and conclusions of the research in this thesis. Also, this chapter includes contributions and future research directions. 


\section{Chapter 2}

\section{Literature Review}

This chapter starts by describing the basic components of a wind turbine. It then presents the process of lightning phenomenon and their incidence on the WTGs. A discussion on lightning discharge current waveforms and parameters is presented. This chapter also describes various lightning discharge current waveform models and the parameters documented in the literature. Also, an introduction to the lightning protection systems for the WTGs is presented. A discussion on the WTG grounding system is also given, along with an overview of the soil resistivity measurements and modelling required for a WTG grounding system.

\subsection{Basic components of a wind turbine genera- tor}

The basic components of a WTG system are shown in Figure 2.1 [39] and outlined as follows [1]:

1. The Blades

- New generation WTGs are equipped with 3 blades, usually made of glass fibre reinforced plastic (GFRP) [40]. The use of 


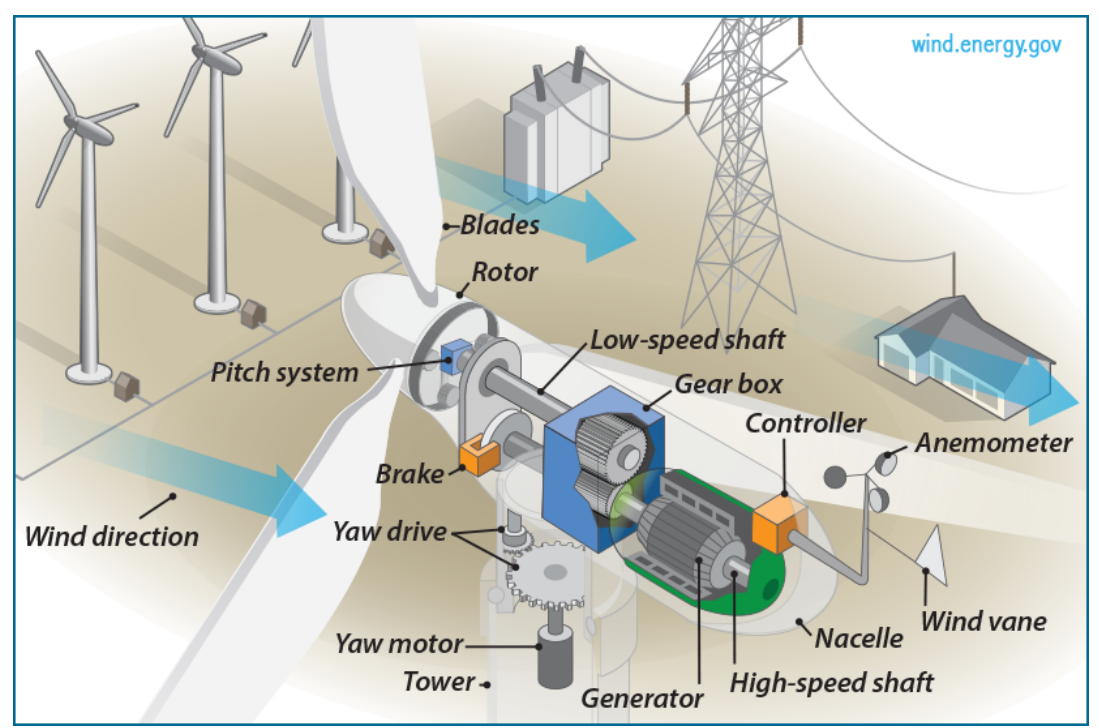

Figure 2.1: Wind turbine generator components [39].

GFRP to manufacture the blades gives them high mechanical strength with a light weight [1].

2. The Hub

- The major components in the hub are the pitch drives and battery boxes [41]. Pitch drives control the blades and their angle to the wind direction to get the optimum power from the wind. Battery boxes are used to provide backup power supply to the pitch drives [42].

3. The Tower

- The tower is the backbone of a large WTG. The tower houses the doubly-fed induction generator (DFIG) panel, power and control panels consisting of protection equipment, relays, measurement transformers, filter units, an auxiliary transformer and an uninterruptible power supply (UPS) unit [42]. 
4. The Nacelle

- The nacelle is the heart of megawatt-class WTGs. Large parts of the electrical and electronic systems such as the generator, controller, and yaw drives are housed in the nacelle. The nacelle is mounted on yaw bearings which in turn are placed on the tower. The yaw system is responsible for the rotation of the nacelle to face the wind direction [42].

\subsection{Lightning}

The Lightning process starts with the formation of thunderstorm clouds or cumulonimbus clouds [43]. The electrical model of these clouds is usually represented as a vertical tripole structure [44], which contains a main negative, main positive and a lower positive charge centre [44], as shown in Figure 2.2 [28]. The values of the charges vary for different models.

Lightning is an atmospheric discharge of current and carries a huge magnitude of current in the range of kilo amperes [45]. Lightning can be classified into two main types, upward and downward initiated. These are also known by the names cloud-to-ground and ground-to-cloud lightning [46]. These two forms of lightning can be further subdivided into positive and negative polarity, the polarity being that of the charge transferred from the cloud to the ground [44] as shown in Figure 2.3 [44]. A lightning charge transfer process starts at the cloud and moves towards the ground [47]. The charge transfer channel starts with the initiation of a leader or streamer. A self-propagating electrical charge transfer creating an electrical conductivity channel with a conductivity of the order of $10^{4}$ $S m^{-1}$ is called as a leader. The conductive channels with lower electric conductivity are termed as streamers [44]. 


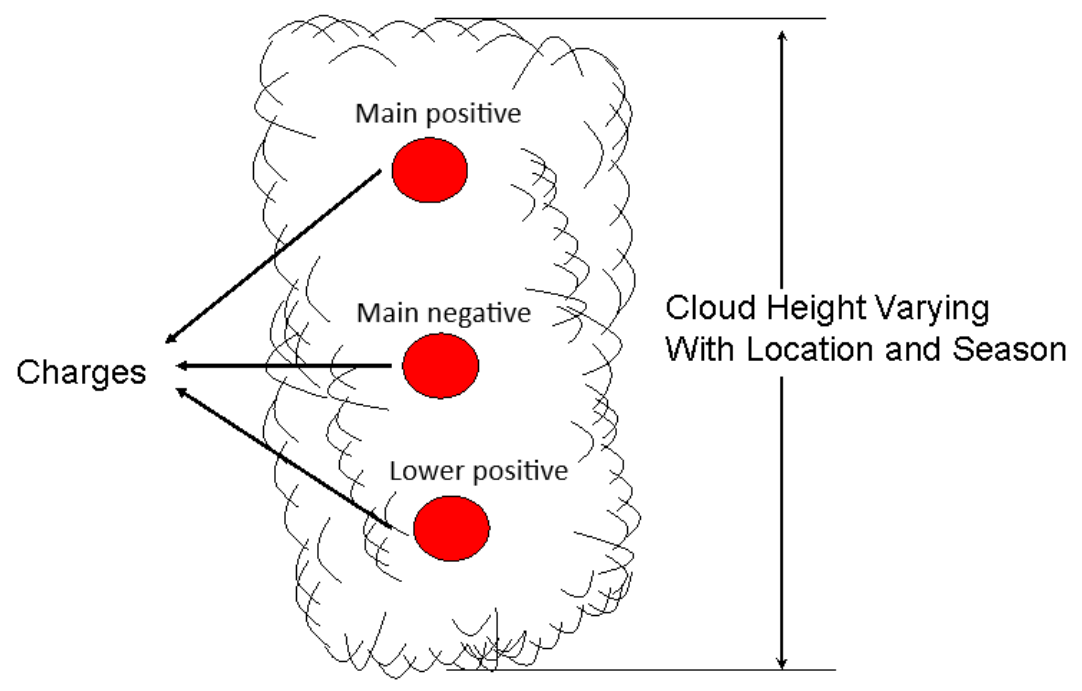

Figure 2.2: Tripole charges in a cloud [28].

\subsection{Incidence of lightning strikes on wind tur- bines}

The impact of lightning strikes, lightning damages and direct and indirect effects of lightning currents on WTGs are discussed in [48], [49]. Damages to WTGs by lightning is currently one of the main sources of WTG insurance claims and downtime [48]. The wind turbine structures can easily attract the wrath of storms and the rotation of the blades may also trigger lightning and result in a considerable increase in the number of strikes to a WTG [50-52]. Lightning damage is the single largest cause of unplanned downtime in WTGs, and that downtime is responsible for the loss of countless megawatts of power generation [48].

The annual failure rate of damaged electrical components due to lightning flashes can be estimated using the procedure proposed in [24]. This method uses the attractive radii method [53], [54] to calculate the incidence 


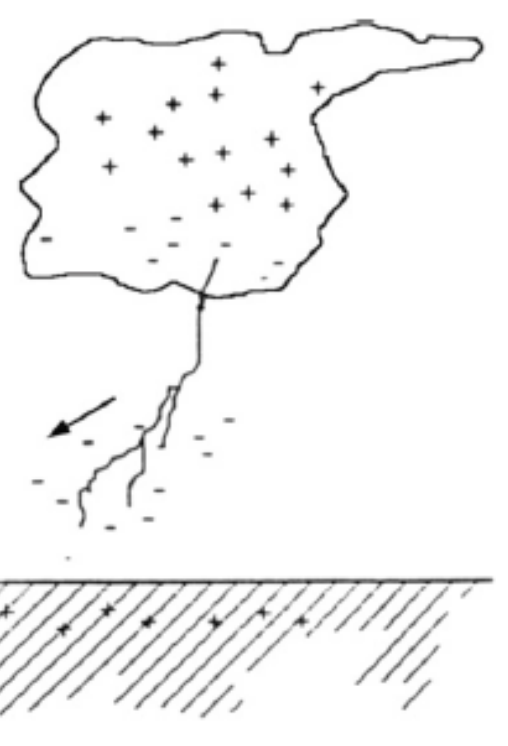

(a) Downward lightning negatively-charged leader.

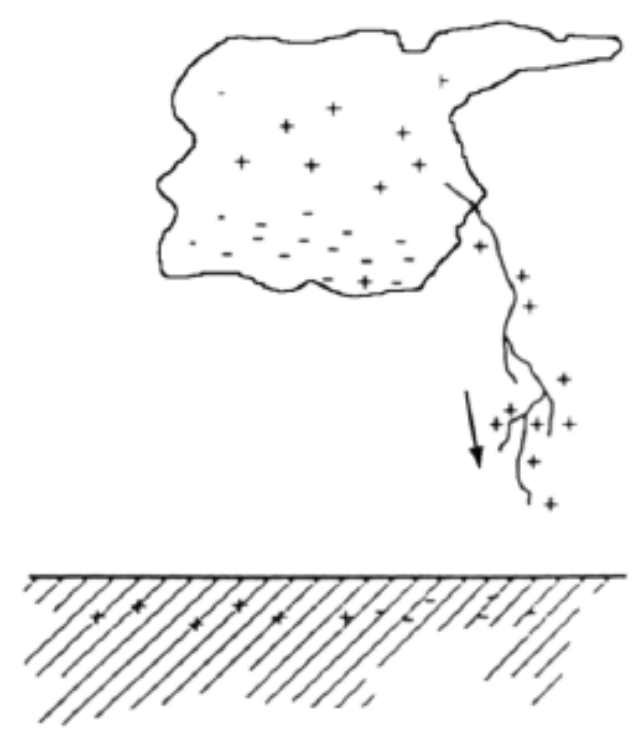

(c) Downward lightning positively-charged leader.

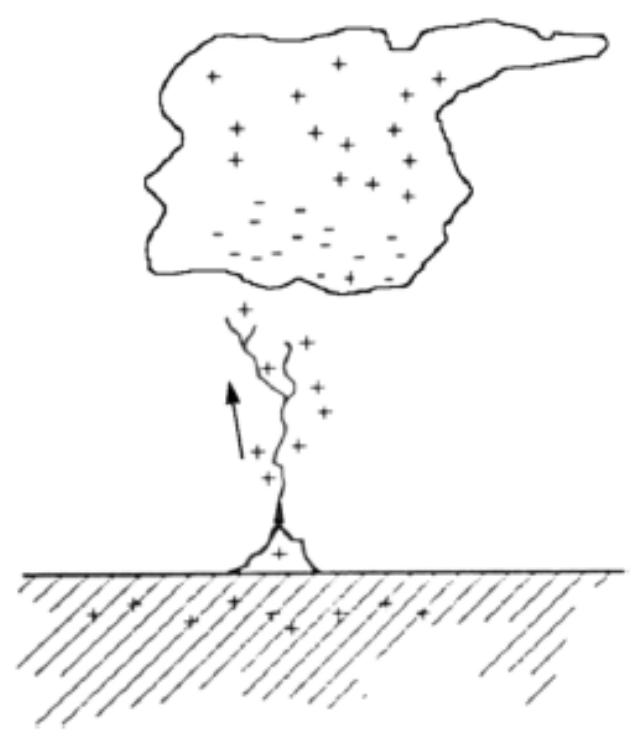

(b) Upward lightning positively-charged leader.

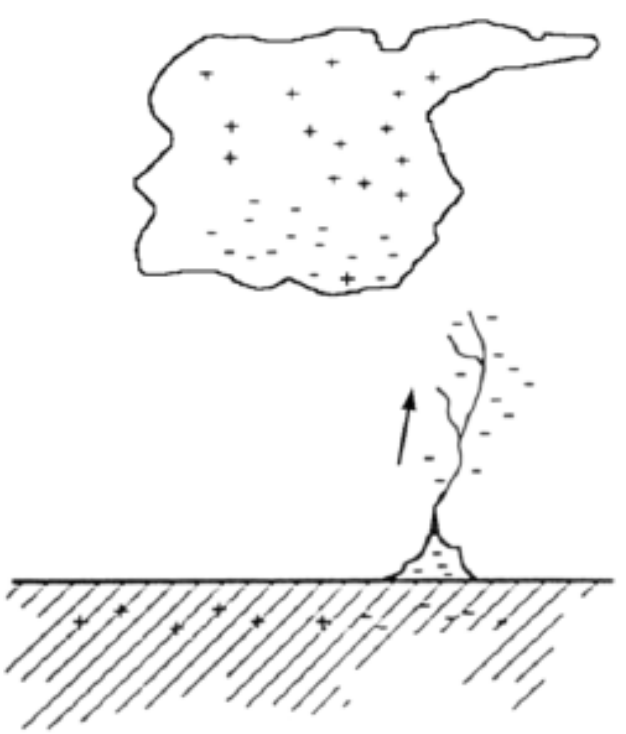

(d) Upward lightning negatively-charged leader.

Figure 2.3: The four types of cloud-to-ground lightning flashes as defined from the direction of leader propagation and the charge on the initiating leader [44]. 
of lightning strikes. Also, this method uses an exact method to evaluate the effective height of the wind turbines. The number of failures per annum of WTG electrical components can be evaluated by Equation 2.1 [24].

$$
F_{W T C}=N_{d} \int_{I_{F_{W T d}}}^{\infty} f(I) d I+N_{u} \int_{I_{F_{W T u}}}^{\infty} f(I) d I
$$

where $I_{F_{W T d}}$ is the minimum magnitude of downward lightning discharge current, $I_{F_{W T u}}$ is the minimum magnitude of upward lightning discharge current, $N_{d}$ is the expected number of downward lightning flashes, $N_{u}$ is the expected number of upward lightning flashes, and $f(I)$ is the probability density function to approximate the lightning discharge current parameters I. This method estimated a $10 \%$ failure rate per year for a ground flash density of 15 and a WTG height of $150 \mathrm{~m}$ on flat terrain, whereas the failure rate increases to $20 \%$ for a WTG located on elevated terrain. The IEC method predicted twice the failure rates compared to the proposed method. This method predicted a lower failure rate of WTGs compared to IEC 61400-24 [17]. The proposed method in [24] accounts for the occurrence of both upward and downward lightning strikes. However, the contribution from the upward lightning strikes was negligible due to their relatively small amplitudes [24].

A wind turbine on flat ground collects lightning strikes which otherwise will strike nearby ground. Lightning strikes on WTGs can be both downward and upward initiated lightning [55]. However, there is still ambiguity with the process of upward initiated lightning strike on WTGs due to the rotating blades. However, it is estimated that there is a high possibility of upward initiated lightning for modern WTGs [56]. Furthermore, carbon reinforced plastics used in the blades will add to the lightning related damages in a WTG [57].

To evaluate upward lightning strikes it is important to calculate the effective height of a WTG. The effective height does not always represent the actual height of a structure above the ground [58]. The effective height depends on the actual height of a structure, as well as topography and 
other effects [56]. The importance of an empirical expression to determine the effective height based on the measurement data obtained on WTGs was explored in [56] and found that the upward lightning strikes possess the similar characteristics of the sub-sequent lightning strikes. In addition, this research indicates that the upward initiated lightning strikes have predominant role in the lightning incidence on WTGs. Also, the rotation of the blades may have a considerable influence on the number of upward initiated strikes to the blades of large WTGs as these may be triggering their own lightning [57]. The occurrence of simultaneous lightning strikes to several WTGs in a wind farm was analysed in [59]. In this study of five events investigated, the cloud to ground (CG) lightning triggering the upward discharges had considerably high current. It was noted that simultaneous discharges at the WTGs caused by nearby lightning may occur frequently. Therefore, the expected number of lightning strikes on WTGs is likely underestimated. The repeated low-current upward lightning and aborted leaders from the WTG have to be considered in lightning protection system design as they may lead to premature degradation of the turbine components, in particular the electrical and electronic devices that are highly vulnerable to electromagnetic pulses. The failure of components due to lightning strikes may not be due to single event lightning strikes and can be from multiple lightning strikes [56,59-62].

\subsection{Lightning current waveforms}

A lightning model is a mathematical model to reproduce certain aspects of the physical processes involved in lightning discharge [44]. Lightning models are validated by comparing with the measured data where available.

In general, the wave shape of a return stroke current which is an impulse current is as shown in Figure 2.4 [63]. The return stroke current waveform consists of a rising front and slow decay tail. To analyse the ef- 
fects of the lightning strike, it is essential to represent lightning discharge current by an analytical expression. There are many models available to represent the lightning current wave shapes [63-71]. Most of the models are based on an exponential function.

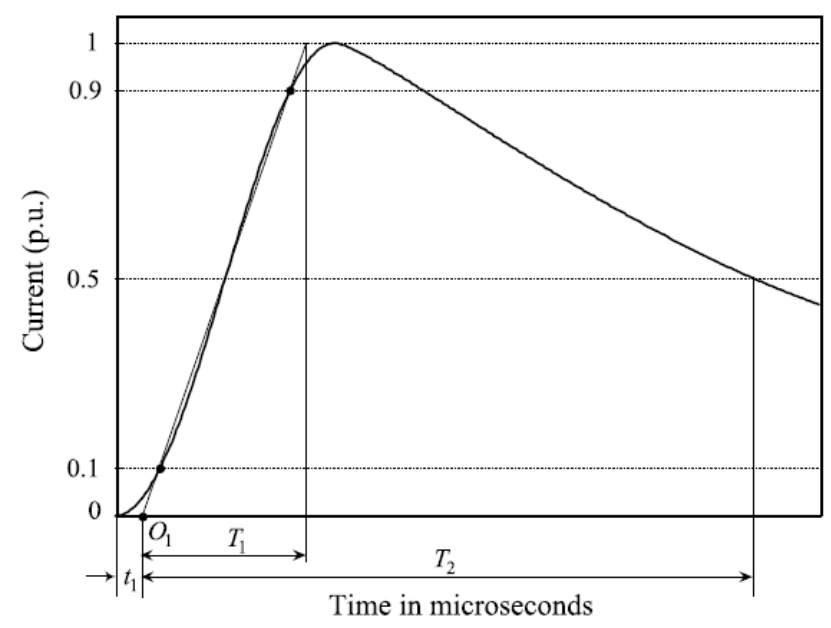

Figure 2.4: Waveform of the standard lightning discharge current [63]. where $T_{1}$ is front time of impulse current, $T_{2}$ is time to half value on the tail of impulse current, $O_{1}$ is virtual origin of impulse current and $t_{1}$ is the time to virtual origin.

Initially, the return stroke current waveform was simulated by a double exponential waveform [72] represented by Equation 2.2

$$
i(t)=c \cdot I_{m} \cdot\left(e^{-a t}-e^{-b t}\right)
$$

where $a, b$, and $c$ are constants which depend on the values of $T_{1}$ and $T_{2}$ shown in Figure 2.4 and $I_{m}$ is the peak value of the lightning discharge current. This Equation 2.2 is not in agreement with the experimentally measured lightning current waveforms as this achieves the maximum rate of current rise at time $\mathrm{t}=0$ [73].

A lightning current return stroke current waveform proposed by Heidler in [64] is a relatively simple function given by Equation 2.3 


$$
\begin{aligned}
i(t) & =\frac{I_{m}}{\eta} \cdot \frac{\left(t / \tau_{1}\right)^{n}}{1+\left(t / \tau_{1}\right)^{n}} \cdot e^{-t / \tau_{2}} \\
\eta & =e^{-\left(\tau_{1} / \tau_{2}\right) \cdot\left(n \cdot \tau_{2} / \tau_{1}\right)^{1 / n}}
\end{aligned}
$$

where $\eta$ is a correction factor of peak current, $n$ is the current steepness factor, $\tau_{1}$ and $\tau_{2}$ are time constants that determine the current rise time and decay time and $I_{m}$ is the peak value of the lightning discharge current.

Another lightning return stroke current model proposed in [73] is a two-component current waveform that provides simple formulae to calculate the parameters, is given by Equation 2.4. Moreover, this waveform is in good agreement with the other standard models [64].

$$
\frac{i(t)}{I_{m}}= \begin{cases}i_{1}(t)=\left[1-\left(1-t / t_{m}\right)^{k}\right]^{n} & \text { for } \quad 0 \leq t \leq t_{m} \\ i_{2}(t)=e^{-(\tau / t) \cdot\left(t / t_{m}-1\right)^{2}} & \text { for } \quad t \geq t_{m}\end{cases}
$$

where $I_{m}$ is the peak value of the lightning discharge current, $t$ is the time, $t_{m}$ is the time from current zero to peak, $\tau$ is the time constant, $n$ and $k$ are the control parameters of the current waveform shape.

$$
\begin{gathered}
t_{m}=\frac{0.8 * T_{1}}{\sqrt[k]{1-0.1^{1 / n}}-\sqrt[k]{1-0.9^{1 / n}}} \\
t_{1}=\left(1-\sqrt[k]{\left.0.1^{1 / n}\right)^{*}} t_{m}-0.1^{*} T_{1}\right. \\
\tau=\frac{\ln 2 *\left(t_{1}+T_{2}\right)}{\left(\frac{t_{1}+T_{2}}{t_{m}}-1\right)^{2}}
\end{gathered}
$$

The methodology considered for this thesis consists of frequency decomposition of the time-domain lightning discharge current waveform and evaluation of the electromagnetic field response in the frequency domain. The analysis of frequency response is adequate for this research, and hence, electromagnetic field response in the time-domain is not included in the thesis. The transient behaviour of the WTG earthing system 
subjected to lightning discharge currents is evaluated in the frequency domain. A double exponential lightning discharge current waveform with different lightning protection level parameters [17] is used in this research.

\subsubsection{Lightning discharge current parameters}

The four lightning protection levels of the IEC 62305-1 [63] are associated with various lightning discharge current parameters [17]. The important parameters required for dimensioning, analysis and testing of the lightning protection system are [17], [74]: peak current, specific energy, charge transferred, maximum current steepness, rise time and fall time. The peak current determines the heating of the conductive parts and the peak potential distribution in a WTG grounding system. The specific energy is important in understanding the thermal heating of the down conduction system in a LPS. The increased value of charge transfer results in melting and erosion of the system. The high $\frac{\mathrm{d} i}{\mathrm{~d} t}$ represents the rapid change of current with time and will induce high voltage in the nearby cables. The rise time and fall time of the lightning discharge current determine the frequency components in the system.

There are several lightning discharge current parameters in the literature with varied rise time, fall time, peak current magnitude and charge transfer $[17,74-76]$. However, the negative first strokes are considered to create worst stress [75]. The first negative stroke parameters from the standard IEC 61400-24 [17] are considered in this research, as depicted in Figure 2.5.

\subsection{Lightning protection system of wind turbine generators}

The effects of lightning strikes on WTGs can be classified into two categories: direct and indirect effects $[77,78]$. Direct effects of lightning strikes 


\begin{tabular}{|c|c|c|c|c|c|c|}
\hline \multicolumn{3}{|c|}{ First short positive stroke } & \multicolumn{4}{|c|}{ LPL } \\
\hline Current parameters & Symbol & Unit & 1 & II & III & IV \\
\hline Peak current & $I$ & kA & 200 & 150 & \multicolumn{2}{|c|}{100} \\
\hline Short stroke charge & $Q_{\text {short }}$ & C & 100 & 75 & \multicolumn{2}{|c|}{50} \\
\hline Specific energy & $W / R$ & $\mathrm{MJ} / \Omega$ & 10 & 5,6 & \multicolumn{2}{|c|}{2,5} \\
\hline Time parameters & $T_{1} / T_{2}$ & $\mu \mathrm{s} / \mu \mathrm{s}$ & \multicolumn{4}{|c|}{$10 / 350$} \\
\hline \multicolumn{3}{|c|}{ First short negative stroke ${ }^{a}$} & \multicolumn{4}{|c|}{ LPL } \\
\hline Peak current & $I$ & kA & 100 & 75 & \multicolumn{2}{|c|}{50} \\
\hline Average steepness & $\mathrm{d} t / \mathrm{d} t$ & $\mathrm{kA} / \mu \mathrm{s}$ & 100 & 75 & \multicolumn{2}{|c|}{50} \\
\hline Time parameters & $T_{1} / T_{2}$ & $\mu \mathrm{s} / \mu \mathrm{s}$ & \multicolumn{4}{|c|}{$1 / 200$} \\
\hline \multicolumn{3}{|c|}{ Subsequent short stroke ${ }^{a}$} & \multicolumn{4}{|c|}{ LPL } \\
\hline Current parameters & Symbol & Unit & 1 & II & III & IV \\
\hline Peak current & $I$ & kA & 50 & 37,5 & \multicolumn{2}{|c|}{25} \\
\hline Average steepness & $\mathrm{d} t / \mathrm{d} t$ & $\mathrm{kA} / \mu \mathrm{s}$ & 200 & 150 & \multicolumn{2}{|c|}{100} \\
\hline Time parameters & $T_{1} / T_{2}$ & $\mu \mathrm{s} / \mu \mathrm{s}$ & \multicolumn{4}{|c|}{$0,25 / 100$} \\
\hline \multicolumn{3}{|c|}{ Long stroke } & \multicolumn{4}{|c|}{ LPL } \\
\hline Current parameters & Symbol & Unit & 1 & ॥ & III & IV \\
\hline Long stroke charge & $Q_{\text {long }}$ & $\mathrm{C}$ & 200 & 150 & \multicolumn{2}{|c|}{100} \\
\hline Time parameter & $T_{\text {long }}$ & s & \multicolumn{4}{|c|}{0,5} \\
\hline \multicolumn{3}{|c|}{ Flash } & \multicolumn{4}{|c|}{ LPL } \\
\hline Current parameters & Symbol & Unit & 1 & II & III & IV \\
\hline Flash charge & $Q_{\text {flash }}$ & C & 300 & 225 & \multicolumn{2}{|c|}{150} \\
\hline
\end{tabular}

Figure 2.5: Maximum values of lightning parameters [17]. 
range from burning and erosion to damage of the WTG structure due to arc attachment. Indirect effects are due to the electromagnetic fields generated by lightning discharge currents and can include transient overvoltages, currents, and electromagnetic interference (EMI) [79-81]. The electrical characteristics of lightning discharge current, namely, the peak current, rate of current rise and attenuation, specific energy, charge transfer, and number of strikes determine the severity of the direct and indirect effects $[17,82,83]$. The most vulnerable parts for WTGs due to lightning strikes are the blades, followed by the nacelle and the hub [80,84-86]. Depending on the hub height, the tower could also be endangered by lightning strikes [80]. Thus the target of the lightning protection system is to achieve effective protection of the electrical systems, control systems, and the general WTG structure.

Lightning protection systems (LPSs) are used to protect WTGs from direct lightning strikes and the indirect effects of electromagnetic fields [87]. Lightning protection systems are designed based on a risk assessment according to the standard IEC 61400-24 [17].

A lightning protection system consists of an external LPS, an internal LPS, and the earthing system [88, 89]. According to IEC 62305 [88], the main components of an LPS for any structure [90] can be outlined as shown in Figure 2.6 [90].

The functions of the external lightning protection system are to:

- Intercept direct lightning strikes via an air-termination system.

- Safely conduct the lightning current to ground via a down-conductor system.

- Distribute the lightning current in the ground via an earthtermination system.

The function of the internal lightning protection system is to prevent dangerous sparking inside the structure [91]. This is achieved by establishing an equipotential bond or maintaining a separation distance between 


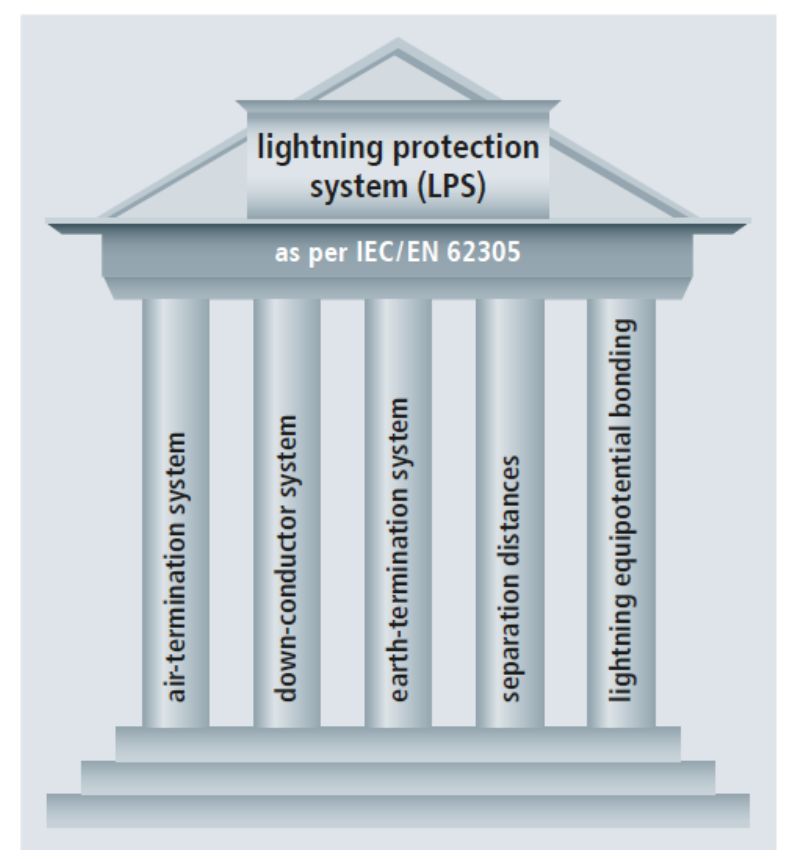

Figure 2.6: Components of a lightning protection system [90].

the components of the lightning protection system and other electrically conductive elements inside the structure [92,93].

The main reason for the use of lightning equipotential bonding is to reduce the potential differences arising due to the lightning discharge currents. In order to reduce it, all isolated conductive parts of the installations are connected by means of conductors or by means of surge protective devices (SPDs) [94-97].

The external lightning protection system of a WTG consists of:

- Air-termination and down-conductor systems in the rotor blades.

- Air-termination systems for protecting the nacelle and the hub.

- Air-termination and down conductor systems as the tower.

- An earth-termination system, consisting of a foundation earth electrode and a ring earth electrode. 
The internal lightning protection system of a WTG consists of:

- Earthing and equipotential bonding measures.

- Spatial shielding and separation distance.

- Cable routing and cable shielding.

- Installation of coordinated surge protective devices.

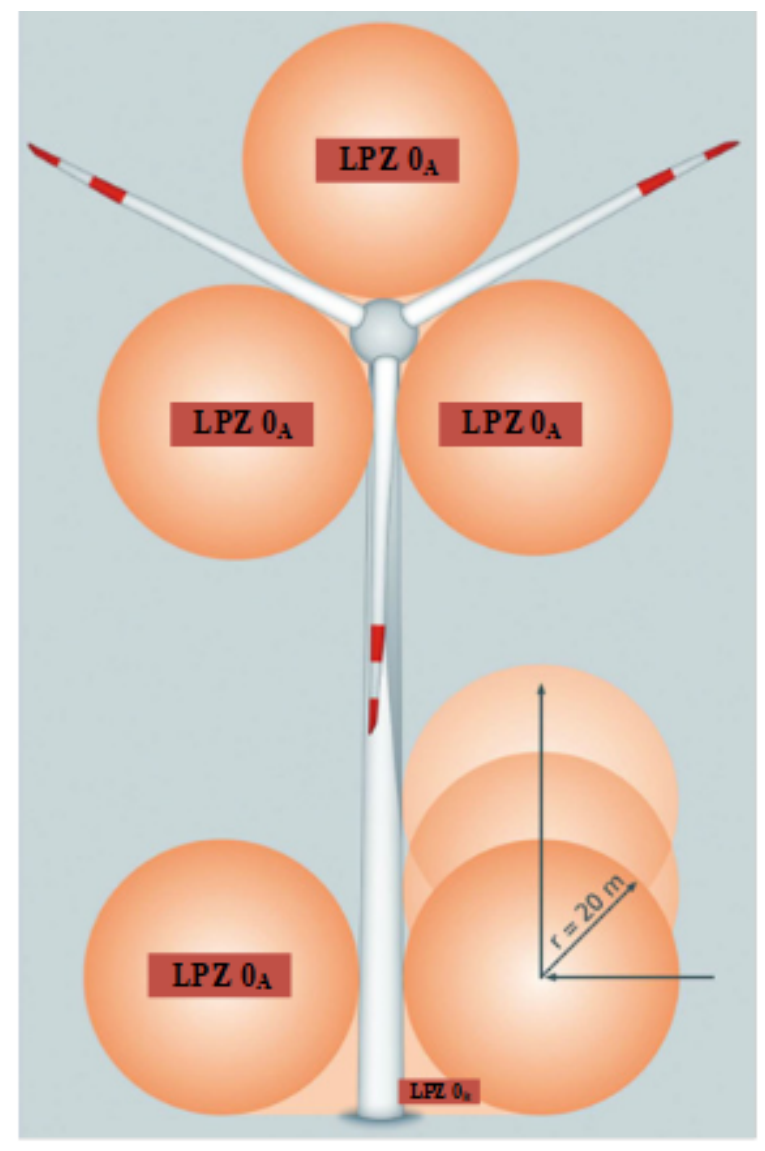

Figure 2.7: Application of rolling sphere method to a wind turbine [100].

The design of lightning protection systems should take into account the risk of lightning flashes striking or damaging the WTG structure. The 
lightning risk assessment of the WTGs is done in accordance with IEC 61400-24 [17,98] and IEC 62305-2 [99]. The lightning protection system of WTGs shall be designed according to LPL-1 unless the risk analysis dictates a lower lightning protection level. The protection system designed according to LPL-1 has the highest protection capability. The basis of LPL1 is an application of the rolling sphere method to identify the parts of the structure exposed to direct lightning strikes as shown in Figure2.7 [100].

All types of lightning flashes generate lightning electromagnetic pulses (LEMP) influencing the electrical and control systems [101, 102]. LEMP protection measures (LPMS) are implemented to avoid failure of these systems. The effective protection of the electrical and control system of a WTG against LEMP is normally designed according to IEC 62305-4 [103], using the concept of lightning protection zones (LPZ) with special measures against LEMP [104-106]. These measures include earthing/grounding, bonding, magnetic and electrical shielding, line routing, surge protection devices (SPDs), adequate EMC immunity levels for systems and devices and insulation [107-110].

The IEC 61400-24 standard recommends the lightning protection system of a WTG be designed to lightning protection level (LPL) 1 unless a risk analysis demonstrates that a lower LPL is sufficient.

\subsubsection{Lightning protection zones}

Lightning protection zones (LPZs) are spaces inside and outside a structure, which describe the grade of electromagnetic influence [111], caused by lightning and electromagnetic disturbances [48]. The IEC 61400-24 [17] standard defines the LPZs and their influence grades for WTG installations as shown in Table 2.1.

The defined electromagnetic environment depends on the immunity of the electrical equipment used in the zone. The lightning protection zone concept restricts the conducted and radiated electromagnetic interference 
Table 2.1: Lightning protection zones [63].

\begin{tabular}{|l|l|l|l|}
\hline \multirow{2}{*}{ Lightning protection zone (LPZ) } & Exposure threats & \\
\cline { 2 - 4 } & Lightning flash & $\begin{array}{l}\text { Lightning current or } \\
\text { induced current }\end{array}$ & Electromagnetic field \\
\hline LPZ $0_{A}$ & Yes & Full & Full \\
\hline LPZ $0_{B}$ & No & Partial & Full \\
\hline LPZ 1 & No & Limited & Partial \\
\hline LPZ 2 & No & Reduced below LPZ-1 & Reduced below LPZ-1 \\
\hline
\end{tabular}

at the zone boundaries to set values by using the lightning and surge protection techniques. For this reason, the structure to be protected is subdivided into protection zones [63, 88,99,112].

The grade of electromagnetic influence and lightning currents inside a LPZ decreases with the increasing zone number [113]. Lightning protection zone 0 (LPZ 0) can be subdivided into two zones: LPZ $0_{A}$ and LPZ $0_{B}$. Inside LPZ $0_{A}$, there is a chance of a direct lightning strike resulting in full exposure to lightning currents and electromagnetic fields. In LPZ $0_{B}$, there is no possibility of direct lightning strikes, but partial lightning currents are possible and the full influence of electromagnetic fields can still be felt. There can be no direct lightning strikes to the components and a reduced influence of lightning currents and electromagnetic fields in LPZ 1 and LPZ 2 [114-116].

The rolling sphere method is employed to determine the protection zones with the radius of the sphere determined by the lightning protection level (LPL) [88]. A lightning protection level is a number that represents a set of lightning discharge current parameters not exceeding specific maximum and minimum design values for each level. [63]. The LPLs are used in designing the protection system according the parameters associated with the protection level according to the IEC 61400-24 standard. For example, LPL 1 uses a radius of $20 \mathrm{~m}$. Application of the rolling sphere method is shown in Figure 2.7 [100]. As shown in this figure, an imaginary sphere of radius $20 \mathrm{~m}$ for LPL 1, is rotated around the WTG. The points on that the sphere touches the WTG are considered to be the points 
that can experience a direct lightning strike [117-119]. According to the IEC 61400-24 standard [17], the rolling sphere method can not be used for the rotor blade.

Direct lightning strikes on the WTG can be avoided by installing an air-termination system. Figure 2.8 shows the possible division of a WTG into different lightning protection zones. The division of a WTG into lightning protection zones depends on the design of the WTG and its components [120]. It is imperative that the lightning discharge current parameters that are entered into LPZ $0_{A}$ from the atmosphere can be decreased by effective shielding and surge protective devices at the zone boundaries to protect the electrical and electronic devices and systems inside a WTG from interference [121].

Lightning protection zones of a WTG for LPL 1 can be divided, as shown in Figure 2.8 [100]. This figure depicts the LPZs of a steel tubular tower. In this example, the rotor blades, wind measurement system, lightning rods placed on the nacelle and exterior structure of the WTG tower are classified as LPZ $0_{A}$. LPZ $0_{B}$ contains internal part of blades and external structure of the nacelle and hub. The nacelle and hub internals can be classified as LPZ 1 when their structures are made of metal or contain necessary shielding protection. Inside the electrical cabinets and boxes placed inside LPZ 1 and the cabinet housing made of fully enclosed metal, inside the generator, gearbox and main shaft, and inside tubular steel tower fall in LPZ 2 [91,122-124].

\subsubsection{Grounding system of wind turbine generators}

A low resistance grounding system allows lightning discharge current to flow without large over-voltages $[125,126]$. An increase in grounding resistance can cause a rise in potential in a WTG and in the soil surrounding it [127]. This potential rise in the foundation and the surrounding ground soil could cause an over-voltage of the power, communications, and con- 


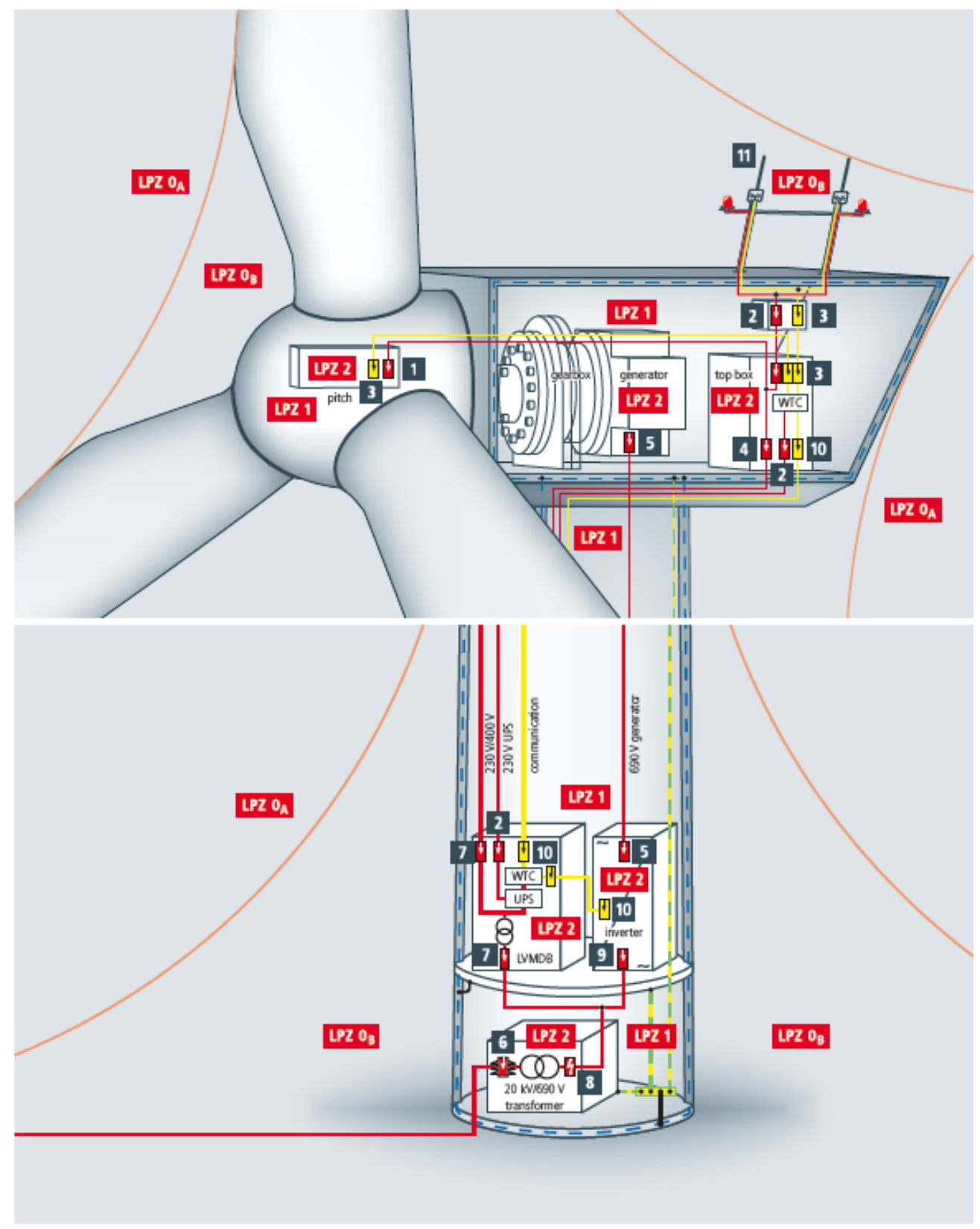

Figure 2.8: Lightning protection zones in a wind turbine [100]. 
trol equipment placed in the WTG and can result in the breakdown or deterioration of the insulation [128]. Further, the personnel who are in the vicinity of a WTG grounding system at the time of lightning strike or power system fault might be at risk [129]. These risks can be mitigated by designing a proper wind turbine grounding system [130].

A lightning strike on a WTG can generate over-voltages not only in the affected WTG, but also in surrounding WTGs [131]. These over-voltages can lead to step and touch voltages in a wind farm. The step and touch voltages of the grounding system of a WTG can be reduced by including additional grounding electrodes and conductors and by connecting foundation reinforcement [132], [133]. Research by Abd-Allah et. al found that these improvements to the grounding system reduced over-voltage by about $95 \%$ and ground potential rise was decreased by about 97\% [134].

The location of an earthing system has a critical effect on the WTG earthing resistance due to the resistivity of the soil [135]. A method was proposed to assess the location of the earthing system to get a low resistance path in [136]. The proposed method depends on the simulation of the electric field as well as the calculation of current density at the ground surface above the earthing system and distribution inside the soil when a WTG is exposed to high voltage. An optimum depth for an earthing system was calculated for the grounding resistance for different soil types.

The goal of a grounding system is to provide a low impedance path for the dissipation of lightning discharge and power system faults into the ground [137]. This task has to be done by limiting the ground potential rise in the surrounding earth of the WTG foundation. The low magnitudes of potential rise reduces the step and touch voltages, which in turn safeguard humans and animals $[138,139]$.

The grounding systems design is generally based on power frequencies $[140,141]$. The resistance is the only component to be considered for designing a grounding system at the power frequencies. However, the grounding system behaviour is different for lightning discharge currents 
due to the high-frequency components [142]. At higher frequencies, the inductive component of the impedance is a dominant factor compared to the resistance. Hence, protection of WTGs from lightning strikes takes priority over power system faults when designing the WTG grounding systems [140].

\subsubsection{Earth electrodes}

An earth electrode is a buried metallic wire in the soil with various geometric dimensions and shapes [143]. The commonly used earth electrodes of a WTG grounding system are: ring, horizontal and vertical electrodes [144].

The wind turbine generator grounding system is designed by connecting the WTG foundation to the earth electrodes. Figure 2.9 depicts the perspective three-dimensional (3-D) view of the wind turbine foundation along with the WTG earthing system and electrodes.

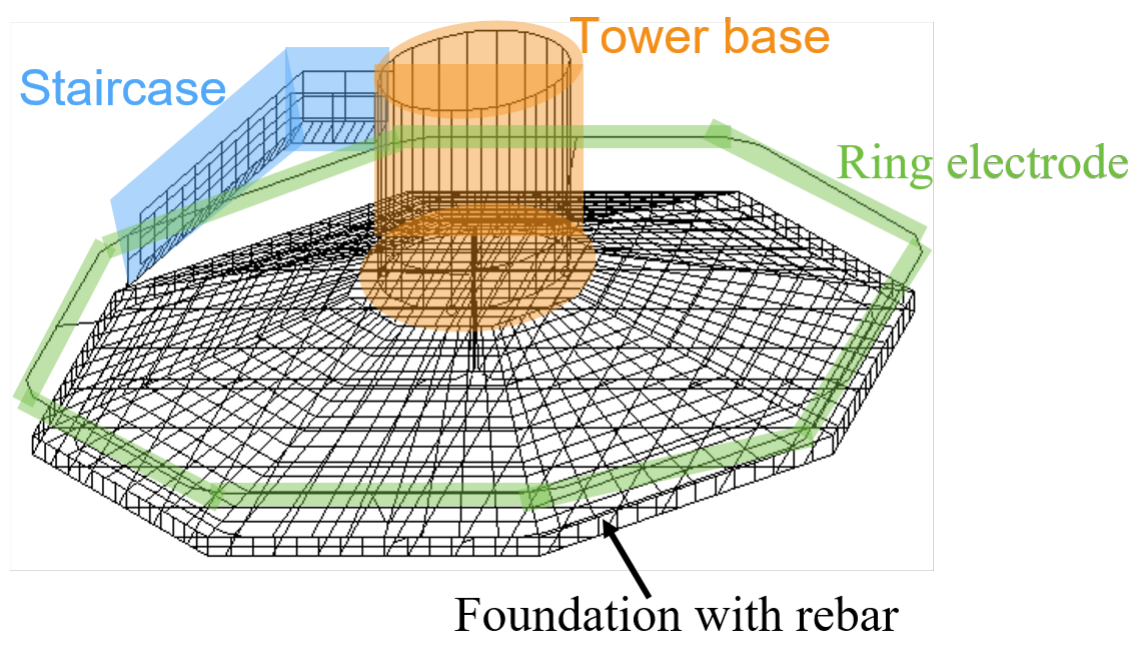

Figure 2.9: Perspective view of wind turbine foundation (not to scale).

The design of a wind farm grounding system begins with the design of an individual WTG grounding system [140]. IEC 61400-24 [17] recom- 
mends a grounding resistance value of less than $10 \Omega$ for an WTG isolated from rest of the wind farm [140]. In addition, the standard recommends using a type-B earthing arrangement, which consists of ring earth electrodes surrounding the WTG foundation in combination with additional radial or vertical earth electrodes [17].

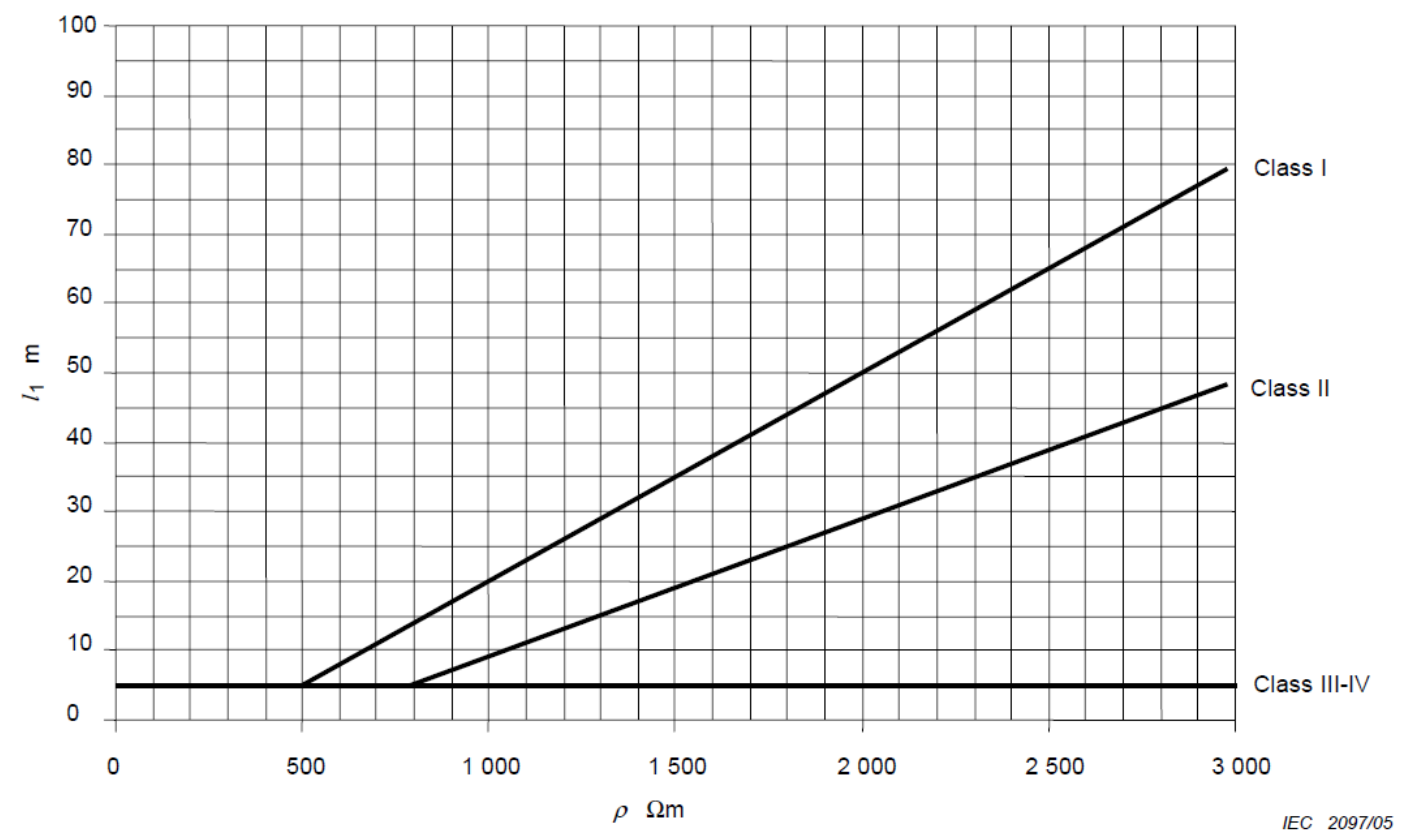

Figure 2.10: Minimum length $\left(l_{1}\right)$ of earth electrode according to the class of lightning protection level [17].

The minimum length of the earth electrode to achieve a resistance of less than $10 \Omega$ is determined predominantly by the soil resistivity of the WTG site [17], as shown in Figure 2.10. Hence the soil resistivity measurement and the interpretation of soil structure is critical in selecting the earth electrode length. The soil resistivity also varies with frequency [145]. Thus, it is important to understand the effect of the frequency dependent soil parameters on the WTG earthing system. The design of earth electrodes in the literature is based on the uniform soil resistivity and does 
not consider other parameters of the earth electrodes viz. dimensions and burial depth of the electrodes. Moreover, the literature is lacking earthing systems design for realistic soil models and neglected the WTG foundation in the design process.

\subsection{Soil Resistivity}

The general mass of earth possess several electrical characteristics: resistivity, permeability and permittivity [146]. Amongst all the properties, the permeability is the only parameter which is constant. Although the dielectric constant varies widely ranging from 1 to 80 , the variation is less pronounced than resistivity [146]. The soil resistivity value ranges widely depending on the composition of the soil [147]. The soil resistivity ranges between 1 and 10k $\Omega \mathrm{m}$ [146].

The resistance is the electrical characteristic of a conductor which prevents the flow of electric current when a potential difference exists across the ends of a conductor. The resistivity is the electrical property of a conductor which defines the resistance of the conductor [148]. The resistance of a conductor $\mathrm{R}$ is calculated using Equation 2.8:

$$
R=\frac{\rho \times L}{A}
$$

where $\rho$ is the resistivity of the conductor material $(\Omega \mathrm{m}), L$ is the length of the conductor (m), and $A$ is the area of cross section in $m^{2}$.

Soil resistivity can be characterized as the resistance between the contrary sides of a 3D square of soil with a side of one meter [148]. The soil resistivity value is dependent on the density, moisture content, temperature, mineral composition and structure of the soil [149]. Moreover, the electrical properties of the soil vary with frequency [145]. The high frequency components of the lightning discharge current change the electrical properties of the soil [34]. 
Table 2.2: Soil resistivity values of typical soils and water at $25^{\circ} \mathrm{C}$ [151]

\begin{tabular}{|l|l|l|}
\hline Type of Soil or Water & Typical Resistivity $(\Omega \mathbf{m})$ & Usual Limit $(\Omega \mathbf{m})$ \\
\hline Sea water & 2 & 0.1 to 10 \\
\hline Clay & 40 & 8 to 70 \\
\hline Ground well and spring water & 50 & 10 to 150 \\
\hline Clay and sand mixtures & 100 & 4 to 300 \\
\hline Shale, slates, sandstone, etc. & 120 & 10 to 100 \\
\hline Peat, loam, and mud & 150 & 5 to 250 \\
\hline Lake and brook water & 250 & 100 to 400 \\
\hline Sand & 2000 & 200 to 3000 \\
\hline Moraine gravel & 3000 & 40 to 10000 \\
\hline Ridge gravel & 15000 & 3000 to 30000 \\
\hline Granite & 25000 & 10000 to 50000 \\
\hline Ice & 100000 & 10000 to 100000 \\
\hline
\end{tabular}

The measurement and modelling of soil resistivity is very important because of its wide variation can effect the design of grounding system [150]. The typical values of soil resistivity are as depicted in Table 2.2 [151].

\subsection{CDEGS software package}

The computation modules used in this research are RESAP, FFTSES, and HIFREQ modules of the Current Distribution, Electromagnetic fields, Grounding, and Soil structure analysis (CDEGS) [152]. The CDEGS software package is a numerical electromagnetic analysis (NEA) [153] tool and solves full-wave Maxwell's equations [154]. The commonly used NEA methods are finite difference time domain (FDTD) method, transmission line matrix (TLM) method, finite element method in time domain (FEMTD) method, method of moment (MoM) method, and partial element equivalent circuit (PEEC) method. The CDEGS software package is based 
on the MoM method.

The CDEGS is a combination of engineering tools integrated to design and analyse problems related to grounding/earthing, electromagnetic fields, electromagnetic interference including AC/DC interference mitigation studies and various aspects of cathodic protection and anode bed analysis [152]. The tools of the CDEGS computes conductor currents and electromagnetic fields generated by an arbitrary network of energized conductors above or below ground for normal, fault, lightning and transient conditions [152]. CDEGS models simple and multi-component conductors, including bare, coated pipes and pipe-enclosed cable systems buried in complex soil structures. The workflow of the CDEGS software package used in this research is depicted in Figure 2.11. 


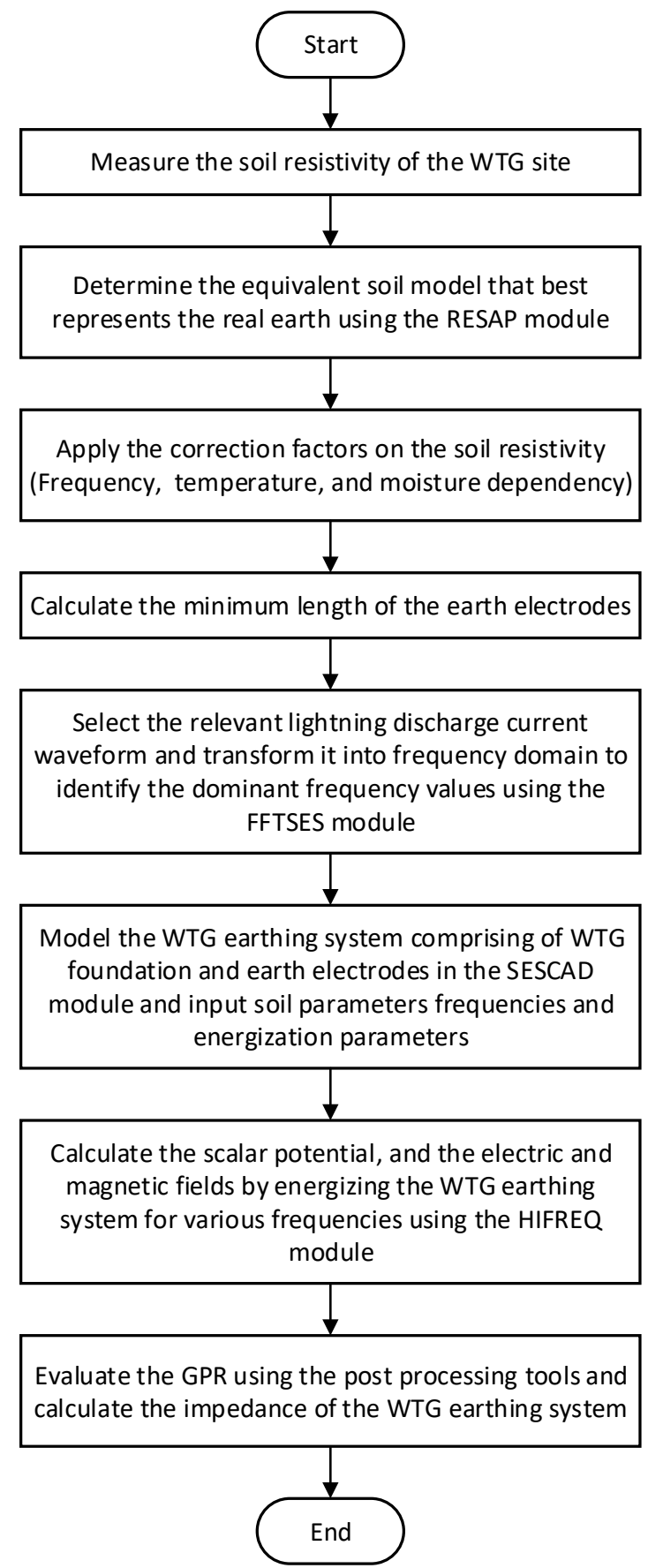

Figure 2.11: Work flow diagram of CDEGS software package. 


\subsection{Chapter summary}

This chapter presents an analysis of the background of the research and works relevant to this study. The background covers the overview of the lightning phenomenon and its incidence to wind turbines. The increased height of the WTGs and their installation sites with a high probability of lightning strikes is resulting in more lightning strikes on WTGs. Also, the height of the WTGs can lead to upward lightning strikes. A review of various lightning discharge current waveforms and parameters are analysed to use the relevant parameters for the simulations of this research. The lightning protection system based on LPL-1 parameters should be considered if a risk assessment of individual WTG is not performed. The peak current and time parameters are important for the design of an earthing system. Since this study focuses on the analysis and design of the earthing system, a review of the WTG grounding system along with the design of earth electrodes is performed. The type-B earthing system is recommended for the WTG earthing system to protection from lightning discharge currents. The methodology of selection of the earth electrodes is not comprehensively presented in the literature. An introduction to soil resistivity and modelling is presented. It is noted that the research gap on the design of earth electrodes for non-uniform soil models should be filled in. 


\section{Chapter 3}

\section{Frequency Domain Analysis of a Wind Turbine Generator Earthing System for Lightning Discharge Currents}

A safe and cost-effective design of a wind turbine generator grounding system requires accurate modelling of local soil resistivity, particularly when wind turbines are spatially distributed across a wide area with various types and features of the soil. In this chapter, three locations at an Australian wind farm were modelled based on measured data. Four soil resistivity models were considered: uniform, multi-layer horizontal, vertical, and exponential variation with depth. Full-wave electromagnetic simulations were performed at different lightning discharge current frequencies to determine the expected ground potential rise and WTG earthing impedance in the event of a lightning strike. The effect of frequency dependent soil parameters on the WTG earthing system is also analysed, along with the effect of foundation rebar on the grounding impedance. Our results showed that an accurate soil resistivity model is critical in the design of a WTG earthing system. 


\subsection{Introduction}

When lightning strikes a WTG, electrical and electronic components embedded in the WTG are prone to failure and damage as a result of the ground potential rise (GPR) caused by electrical surges [155]. Consequently, WTGs should be provided with a low impedance grounding system to ensure safe and reliable operation. Recommendations for effective grounding and lightning protection of WTGs and wind power systems are provided by IEC 61400-24 [17].

An effective grounding system design for WTGs ensures that the equivalent earthing resistance, local ground potential, and step and touch voltages are minimised for safe, reliable and cost-effective operation of the system [156], [17]. Furthermore, robust WTG grounding systems reduce the effects of soil ionization [157]. However, in order to achieve this, a pivotal requirement is an accurate soil resistivity measurement and interpretation to determine the soil structure where WTGs are being located [158].

Data from soil resistivity measurements for WTGs are usually fit into one of the series of standard models [159]. Common models assume either uniform soil resistivity, multi-layer (i.e. step-wise) horizontallyvarying resistivity, multi-layer vertically-varying resistivity or exponential vertically-varying resistivity [160]. The extant literature presents various soil models for the interpretation of soil resistivity measurements at power frequencies and without an actual measurement of local soil resistivity. Thus a detailed comparison of assessments from various soil models with actual field tests and measurement under both power frequencies and frequencies resulting from lightning current energisation are pivotal to accurately represent realistic resistivity values, which need to be known for the safe and effective operation of WTGs [161].

Attempts to address the topic in the literature have so far been piecemeal in nature. Cotton [140] presented a wind farm earthing system with a uniform soil resistivity of $500 \Omega \mathrm{m}$, which is not a representative value 
in practice [156]. Hatziargvriou et al. [142] also analysed a wind farm earthing system with a uniform soil resistivity value of $100 \Omega \mathrm{m}$. Visacro et al. [162] considered the reaction of grounding systems to lightning discharge currents by considering a uniform soil resistivity value. Although Kontargyri et al. [156] presented a detailed study on wind farm earthing systems, it was conducted just at the power frequency without considering lightning discharge current energisation. Kontargyri et al. considered uniform, horizontal two and three-layer soil structures for analysing the wind farm grounding system. Further, Alipio et al. [163] performed an analysis of wind turbine grounding performance for lightning discharge currents with frequency dependent soil parameters. However, this study did not consider the multi-layer soil structures.

A similarity in the existing literature is the assumption of uniform soil resistivity, which is unrealistic in practice as measured soil resistivity can vary both horizontally and vertically [135]. Weather conditions [164], especially humidity levels [165], [166], can also cause a change in the soil resistivity throughout the year. Inaccurate estimation of the dimension and length of earthing electrodes, which are based on the estimated value of soil resistivity, can lead to significant errors in the design of an effective grounding system for a wind turbine. Finally, most of the studies have neglected frequency dependent soil parameters while designing a wind turbine grounding system. It is reported that errors of up to $60 \%$ are observed with fixed values of soil parameters [163].

The IEEE-80 standard [167] recommends the use of a two layer soil model for locations with non-uniform soil resistivity. However, wind turbines are located where soil resistivity varies greatly with different electrode spacing, depth and traverses. Also, wind turbine foundations are about 2-3 meters deep in the soil with a small foundation radius [168], thus making it difficult to interpret soil resistivity to design a safe and cost-effective grounding system. The effect of soil models on the WTG grounding impedance is analysed in this work. Also, the installation of 
earthing electrodes is a critical factor in the WTG earthing system and also addressed in this work.

This study analyses the impact of soil models on the WTG grounding impedance and also addresses the installation of the earthing electrodes in a WTG earthing system. The main contribution of this chapter is to fit the data measures from three different WTG sites in Australia into various soil resistivity models and assess its effects on the WTG earthing impedance. Full-wave electromagnetic simulations were performed using the CDEGS software package [152] to determine the GPR and WTG earthing impedance for different soil structures and lightning discharge current frequencies. Also, the impact of frequency dependent soil parameters on the WTG grounding was analysed. Besides, the effect of earth electrodes installation for a horizontal multi-layer soil model and the effect of WTG foundation metallic components (Rebar) in the earthing system was analysed. Results show that the measured soil resistivity values have a significant effect on the design of a wind turbine earthing system. Additionally, the effect of metallic components on the WTG grounding system is analysed by considering a rebar which share the lightning discharge currents [169].

\subsection{Soil Resistivity Measurement and Interpre- tation}

To illustrate the differences between soil models, a series of soil resistivity measurements from three different sites across Australia are considered. The measurements were obtained using the Wenner method [164]. In this technique four electrodes will be driven into the earth's surface in a straight line at equal spaces. The current circulates among the outer pair of electrodes and voltage is measured across the inner pair for measuring the resistance $R$, as depicted in Figure 3.1. The depth at which the electrodes 
are buried is maintained at a small value in comparison to the spacing among them in order to regard them as points. Measurements were taken

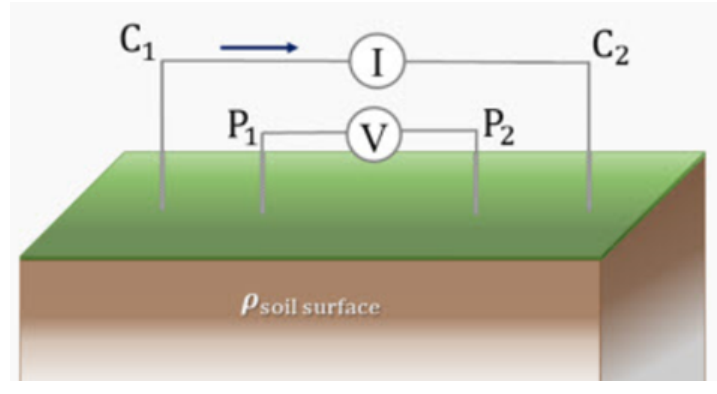

Figure 3.1: Soil resistivity measurement using four point Wenner method [152].

at points along two perpendicular traverses of each site to allow an accurate estimation of the underlying soil structure [170]. Each measurement was repeated three times in quick succession by the same equipment and averaged to ensure the captured data represents the actual conditions at each site on the day and time of measurement. The apparent soil resistivity, $\rho$, was calculated from $R$ using Equation 3.1:

$$
\rho=\frac{4 \times \pi \times a \times R}{1+2 \times \frac{a}{\sqrt{a^{2}+4 \times b^{2}}}-\frac{a}{\sqrt{a^{2}+b^{2}}}}
$$

where $b$ is the depth of the electrode in the ground, and $a$ is the spacing between the electrodes, which was varied between $0.5 \mathrm{~m}$ and $200 \mathrm{~m}$. Data from the soil measurement campaign are summarised in Table 3.1.

\subsubsection{Interpretation of soil resistivity measurements}

The fitting of the measured values to a soil model is critical in the designing of an earthing system. The aim is to derive a soil structure that best represents the actual soil [159].

The most common and easiest model for approximating is the uniform soil model [171]. This model is assumed to have a single layer with an 


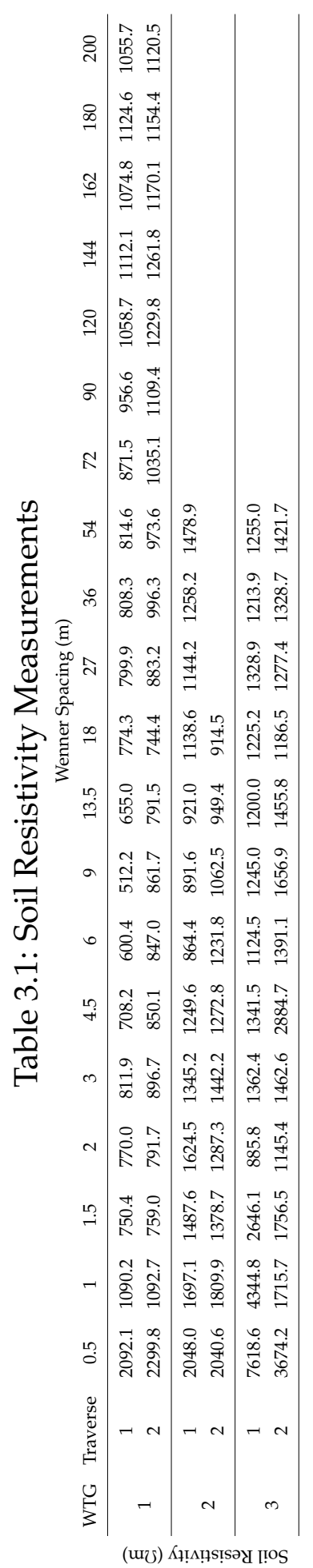


infinite thickness and a constant resistivity value. The uniform soil resistivity $\rho$ is calculated by averaging all the measured resistivity values using Equation 3.2.

$$
\rho=\frac{\rho_{a(1)}+\rho_{a(2)}+\rho_{a(3)}+\ldots+\rho_{a(n)}}{n}
$$

where $\rho_{a(1)}, \rho_{a(2)}, \ldots \rho_{a(n)}$ are the measured apparent soil resistivity values at different spacing of the electrodes and $n$ is the total number of measurements.

The uniform soil resistivity model is seldom a realistic approach due to the tendency of soil resistivity to vary both horizontally and vertically due to soil stratification. The horizontal two-layer soil model is a more sophisticated model, which is a commonly used approximation to represent the soil structure [172].

A two layer soil model is characterised by a top layer with a resistivity $\rho_{1}$ and a thickness $h$, and a bottom layer with $\rho_{2}$ as resistivity and semiinfinite thickness. The change in the soil resistivity at the boundary of the layer is described by means of a reflection factor, $k$, which is defined in Equation 3.3.

$$
k=\frac{\rho_{2}-\rho_{1}}{\rho_{2}+\rho_{1}}
$$

There are several analytical and graphical methods in the literature for determining the parameters of a two layer soil structure. Equation 3.4, presented in IEEE-81 [173] is one such method:

$$
\rho(a)=\rho_{1}\left[1+4 \sum_{n=1}^{\infty}\left(\frac{k^{n}}{\sqrt{1+(2 n h / a)^{2}}}-\frac{k^{n}}{\sqrt{4+(2 n h / a)^{2}}}\right)\right]
$$

here $\rho(a)$ is the apparent soil resistivity and $a$ is the spacing between the electrodes.

In the case of soil with highly non-uniform conditions, a multilayer soil model gives a better approximation of the soil. A three-layer soil model is described by three horizontal layers with soil resistivity of $\rho_{1}, \rho_{2}$ and $\rho_{3}$ and thickness of $h_{1}, h_{2}$ and $\infty$. Additionally, other commonly used soil 
structures are vertical and exponential soil models. When the boundary layer separating the soil resistivity regions is not horizontal, but with an inclination angle of $90^{\circ}$, this is referred to as a vertical two layer soil structure. The apparent resistivity $\rho(a)$ for this model can be calculated from Equation 3.5.

$$
\begin{array}{r}
\rho(a)=\frac{\rho_{1}}{(1-k)}\left[1+k^{2}+\frac{k(k-1)}{\left[4(\sin \omega+h / a)^{2}+\cos ^{2} \omega\right]^{\frac{1}{2}}}\right. \\
\left.-\frac{k(1+k)}{\left[4(\sin \omega-h / a)^{2}+\cos ^{2} \omega\right]^{\frac{1}{2}}}\right]
\end{array}
$$

where $\rho_{1}$ is the resistivity of the region on one side of the vertical layer separation, $\rho_{2}$ is resistivity of the region on the opposite side of the vertical layer separation, $h$ is the distance from the center of the array direction and the vertical separation, and $\omega$ is the angle of the array direction to the vertical line.

Soil structure with an exponentially changing resistivity with depth is referred to as exponential variation with depth soil model. The apparent soil resistivity is represented by Equation 3.6.

$$
\rho(a)=\rho_{2}-\left(\rho_{2}-\rho_{1}\right) \exp (-\lambda a)(2-\exp (-\lambda a))
$$

where $\rho(a)$ is measured apparent resistivity, $\rho_{1}$ is resistivity on the soil surface, $\rho_{2}$ is the resistivity of the deepest part, and $\lambda$ is a scaling coefficient.

The RMS error was used in the evaluation of the soil model fitting from the measured values. The RMS error provides a quantitative indication of the agreement between the measurements and the proposed soil models.

\subsection{Frequency dependent soil parameters}

The behaviour of an earthing system exposed to lightning discharge currents is a complicated issue. The soil parameters, particularly resistivity 
and permittivity, greatly affect the response of the earthing system [145]. Generally, the permeability of the soil has identical values to that of air. However, experimental results show that the resistivity and relative permittivity exhibit strong dependency on frequency [145]. This is critically important for lightning discharge currents having high frequency components. It has been reported that neglecting the frequency dependency of soil resistivity and relative permittivity results in ground potential rise being over estimated by up to $60 \%$ [163]. This is due to an historic lack of accurate formulations to represent the frequency dependency of soil parameters. Recently Alipio et al. [163] proposed frequency dependent soil parameter formulae, mentioned in Equations 3.7 and 3.8, which are helpful in designing a proper grounding system of WTGs from a lightning protection viewpoint.

$$
\begin{gathered}
\rho=\rho_{0}\left\{1+\left[1.2 \times 10^{-6} \cdot \rho_{0}^{0.73}\right] \cdot\left[(f-100)^{0.65}\right]\right\}^{-1} \\
\epsilon_{r}=7.6 \times 10^{3} f^{-0.4}+1.3
\end{gathered}
$$

where $\rho_{0}$ is the soil resistivity at $100 \mathrm{~Hz}, \rho$ is the soil resistivity at required frequency, $f$ is the frequency, and $\epsilon_{r}$ is the frequency dependent relative permittivity of soil.

Equation 3.7 is applicable for the frequency range of $100 \mathrm{~Hz}$ to $4 \mathrm{MHz}$. The soil resistivity for frequencies lower than $100 \mathrm{~Hz}$ should use the value of $100 \mathrm{~Hz}$ and beyond $4 \mathrm{MHz}$ should use the value at $4 \mathrm{MHz}$. Equation 3.8 is applicable for the frequency range of $10 \mathrm{kHz}$ to $4 \mathrm{MHz}$. The relative permittivity for frequencies lower than $10 \mathrm{kHz}$ should use the value of $10 \mathrm{kHz}$ and beyond $4 \mathrm{MHz}$ should use the value at $4 \mathrm{MHz}$.

\subsection{Wind Turbine Generator Earthing}

The earthing system is an important component to maintain the physical integrity of a structure and protect human safety [21]. The literature on 
lightning protection of wind turbines is largely concentrated on the design of wind turbine blades; however, an appropriate earthing system is also critically important in the lightning protection system of a WTG [21].

According to IEC 61400-24 [17], an earthing resistance of less than $10 \Omega$ is required for a WTG before it is connected to the rest of the wind farm. Typically, this is accomplished by the inclusion of the rebar in the WTG foundation, and using a ring earth electrode and additional horizontal and vertical electrodes [17]. It should be noted that IEC 61400-24 [17] recommends interconnecting WTG grounding systems and this can further reduce the low-frequency impedance [174]. A three dimensional perspective view of the WTG foundation modelled for this work is illustrated in Figure 2.9 in Section 2.5.3.

\subsection{Lightning Discharge Current}

The lightning discharge current parameters vary with the lightning protection level (LPL) required for a wind turbine. The required LPL is selected by performing a risk assessment for an individual wind turbine. If the risk assessment cannot be completed for individual wind turbines, the IEC 61400- 24 standard [17] recommends the designing of lightning protection system to LPL-1. Hence, the lightning discharge current parameters of LPL-1 were considered for this analysis.

Time domain lightning discharge current waveforms were converted to frequency domain and simulations were performed in the frequency domain at prominent frequencies of $5 \mathrm{kHz}, 1 \mathrm{MHz}, 4.52 \mathrm{MHz}$, and $10.54 \mathrm{MHz}$. A peak current of $100 \mathrm{kA}$ was used for the simulations. As the lightning discharge currents contain a wide frequency spectrum [175], it is important to understand the significance of individual frequency component. The high-frequency components represent the initial rise time of the lightning discharge currents while the low-frequency components represent the fall time and steady state response. The $5 \mathrm{kHz}$ and $10.54 \mathrm{MHz}$ 
frequencies in the lightning discharge current waveform represents the dominant frequencies at half values during fall time and rise time, respectively. $1 \mathrm{MHz}$ represents the predominant frequency at the peak value of the lightning current and $4.52 \mathrm{MHz}$ was selected due to its proximity to the cut-off frequency $(4 \mathrm{MHz})$ of the frequency-dependent soil parameters [163]. The peak value of lightning discharge current is useful in calculating the potential distribution which in turn used in the evaluation of the step and touch voltages.

\subsection{Results and discussion}

The simulations were performed using different modules of the CDEGS [152] software package. The RESAP module was utilised for soil modelling, and the FFTSES module was utilised to determine the dominant frequencies of the lightning discharge current wave. The HIFREQ module was used in calculating the current, voltage and GPR in the system. HIFREQ is the computation module used in solving transient and steady state problems of the conductors that are buried and aboveground in the frequency domain from zero to hundreds of megahertz. It evaluates the electric and magnetic fields in the air and soil, also conductor and earth potentials, and the current distribution in the earth as well as the conductors. The CDEGS software has been scientifically validated by field tests and compared with research publications for over forty years; it is widely accepted by the industrial and consulting firms world-wide [176]. The software was used to model the complete steel structure of the WTG foundation without considering the effect of concrete and its related resistivity. 


\subsubsection{Soil Models}

The measured soil resistivity values at three wind turbine locations are depicted in Table 3.1. The measurements were performed in two traverses in order to represent soil properties more accurately. These measured values were modelled as different soil structures defined in Section 3.2.1. Table 3.2 depicts the soil structure models and resistivity values for wind turbine location 1, WTG-1. Similar data were calculated for all the wind turbine locations for measured soil resistivities in traverse one, two and a combination of one and two.

\section{Table 3.2: Soil Structure of WTG-1 for Different Soil Models}

\begin{tabular}{lllllll}
\hline Soil Model & $\rho_{1}(\Omega \mathrm{m})$ & Thickness $(\mathrm{m})$ & $\rho_{2}(\Omega \mathrm{m})$ & Thickness $(\mathrm{m})$ & $\rho_{3}(\Omega \mathrm{m})$ & Thickness $(\mathrm{m})$ \\
\hline $\begin{array}{l}\text { Uniform } \\
\text { Horizontal }\end{array}$ & 880.23 & $\infty$ & & & & \\
two layer & 2593.05 & 0.37 & 759.15 & $\infty$ & & \\
$\begin{array}{l}\text { Horozontal } \\
\text { three layer }\end{array}$ & 2593.05 & 0.46 & 614.07 & 16.48 & 1083.15 & $\infty$ \\
$\begin{array}{l}\text { Vertical }^{1} \\
\text { Expontial }\end{array}$ & 706.98 & Left layer & 3310.06 & Right layer & & \\
\hline
\end{tabular}

${ }^{1}$ For vertical soil model: $\rho_{1}$ is resistivity of left layer and $\rho_{2}$ is resistivity of right layer

${ }^{2}$ Exponential coefficient: 0.015 (1/meter)

Table 3.3 presents the RMS percentage error for the soil models of three wind turbine locations. The RMS error provides a quantitative indication of the agreement between the measurements and the proposed soil models. The RMS error is calculated using Equation 3.9.

$$
R M S \operatorname{Error}(\%)=\sqrt{\operatorname{Average}\left(D i^{2}\right)}
$$

$D i$ is the percentage discrepancy between the measured and computed soil resistivity values.

$\mathrm{T} 1, \mathrm{~T} 2$, and T1 \& T2 represent traverse-1, traverse-2 and a combination of traverse- $1 \& 2$, respectively. For WTG-1 and T1, the RMS error 
Table 3.3: Percentage Error of Soil Models

\begin{tabular}{llll}
\hline & \multicolumn{3}{c}{ RMS error (\%) } \\
Soil Model & WTG-1 & WTG-2 & WTG-3 \\
\hline T1-Uniform & 28.02 & 21.08 & 46.08 \\
T1-Horizontal two-layer & 21.41 & 15 & 21.29 \\
T1-Horizontal three-layer & 9.58 & 7.55 & 16.23 \\
T1-Vertical & 19.96 & 23.71 & 22.76 \\
T1-Exponential & 20.04 & 14.43 & 19.42 \\
T2-Uniform & 21.95 & 24.3 & 27.78 \\
T2-Horizontal two-layer & 16.24 & 10.69 & 18.71 \\
T2-Horizontal three-layer & 7.89 & 5.27 & 18.76 \\
T2-Vertical & 16.2 & 9.9 & 24.15 \\
T2-Exponential & 16.33 & 10.24 & 17.96 \\
T1\&T2-Uniform & 26.78 & 25.36 & 37.89 \\
T1\&T2-Horizontal two-layer & 19.88 & 13.34 & 24.24 \\
T1\&T2-Horizontal three-layer & 11.44 & 11.59 & 24.05 \\
T1\&T2-Vertical & 19.82 & 13.16 & 24.35 \\
T1\&T2-Exponential & 19.92 & 13.07 & 23.85 \\
\hline
\end{tabular}


for the uniform soil model is $28.02 \%$, it is $21.41 \%$ for horizontal two-layer soil stratification, $9.58 \%$ for horizontal three-layer soil structure, $19.96 \%$ for vertical and $20.04 \%$ for exponential structures. It can be inferred from the results that the percentage error is maximum for the uniform soil model and minimum for the horizontal three-layer model. The decrease in error ranges from $35 \%$ to $78 \%$.

\subsubsection{WTG earthing impedance variation with soil param- eters}

This section presents the frequency response of the WTG earthing impedance for the changes in soil resistivity and relative permittivity. Figure 3.2 shows the variation in WTG earthing impedance with a change in soil resistivity from $10 \Omega \mathrm{m}$ to $10 \mathrm{k} \Omega \mathrm{m}$, while relative permittivity is kept constant at 10. From the frequency response it can be observed that the WTG earthing impedance for $10 \Omega \mathrm{m}$ increases with frequency up to $4.52 \mathrm{MHz}$ and then a dip is observed at $6 \mathrm{MHz}$. Whereas for higher soil resistivity values of $100 \Omega \mathrm{m}$ and $1000 \Omega \mathrm{m}$, the impedance increases up to a frequency of $3.01 \mathrm{MHz}$ and reduces at the frequency of $6 \mathrm{MHz}$. For $10 \mathrm{k} \Omega \mathrm{m}$ soil resistivity, a decrease in impedance is observed in the initial frequency spectrum before a peak at $3.01 \mathrm{MHz}$, which then reduces to its lowest impedance at $6 \mathrm{MHz}$. However, for all the values of soil resistivity the WTG earthing impedance increases after $6 \mathrm{MHz}$. It is interesting to note that the deviation in impedance is minimal for soil resistivities up to $1000 \Omega \mathrm{m}$ at $10 \mathrm{MHz}$. It is also observed that the inductive component of the impedance decreases with an increase in soil resistivity, as shown in Figure 3.3.

The frequency response of the WTG earthing system is analysed for distinct relative permittivity values of the soil. At lower values of soil resistivity, the change in relative permittivity does not effect the WTG earthing impedance throughout the frequency spectrum. Similarly, there is no 


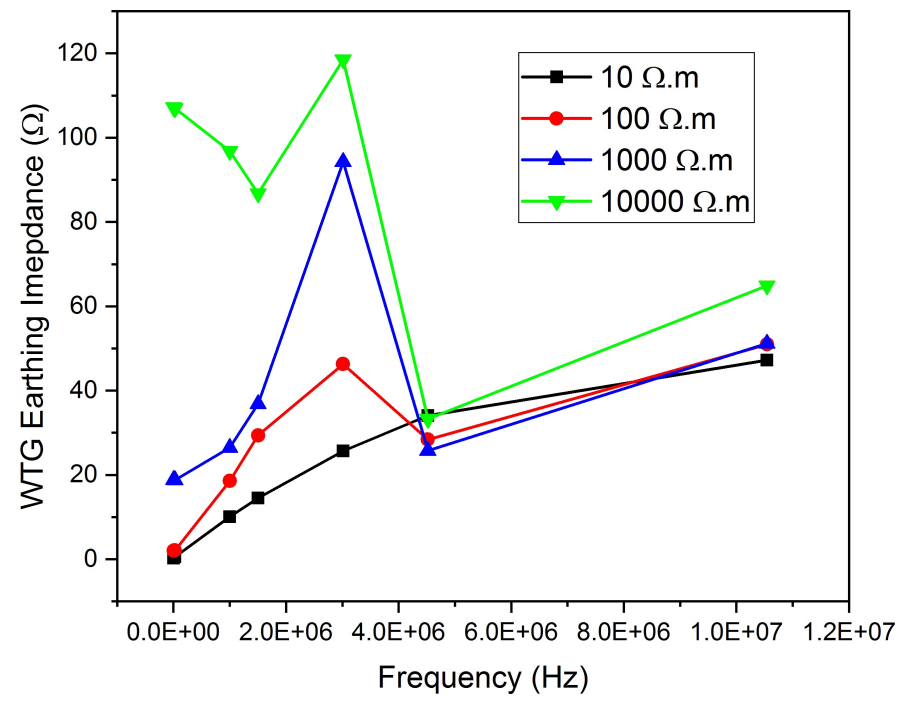

Figure 3.2: Frequency response of the WTG earthing impedance with change in soil resistivity with a constant relative permittivity.

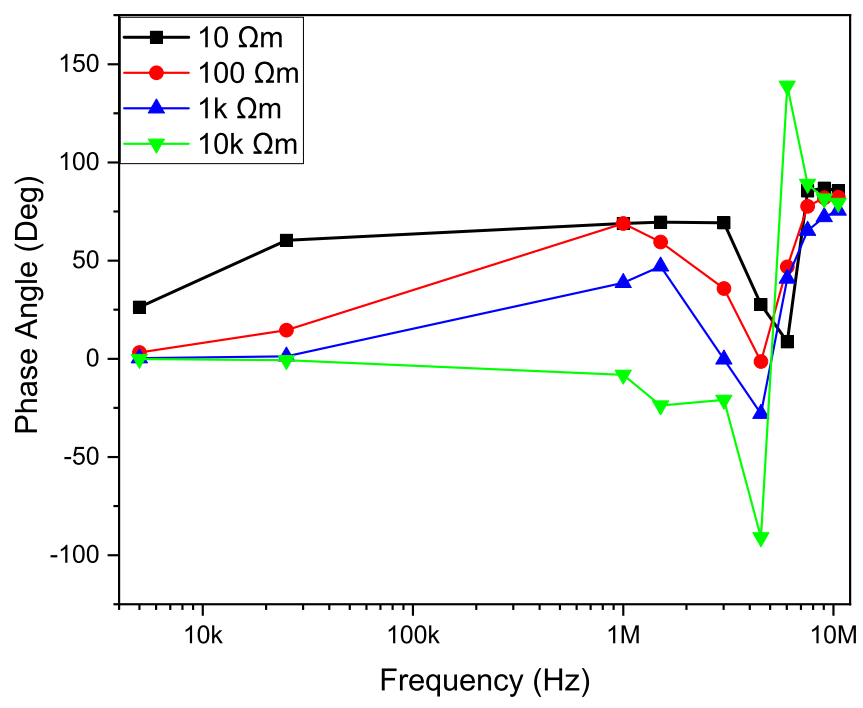

Figure 3.3: Frequency response of the phase angle with change in soil resistivity with a constant relative permittivity. 
change in phase angle with the change in relative permittivity. However, for high soil resistivity value of $10 \mathrm{k} \Omega \mathrm{m}$, the variation in WTG earthing impedance is significant with a variation in relative permittivity, as shown in Figures 3.4 and 3.5. However, there is no common trend observed in the WTG earth impedance change with varying permittivity. Hence, it is important to consider the frequency dependency of the relative permittivity while designing the wind turbine earthing system.

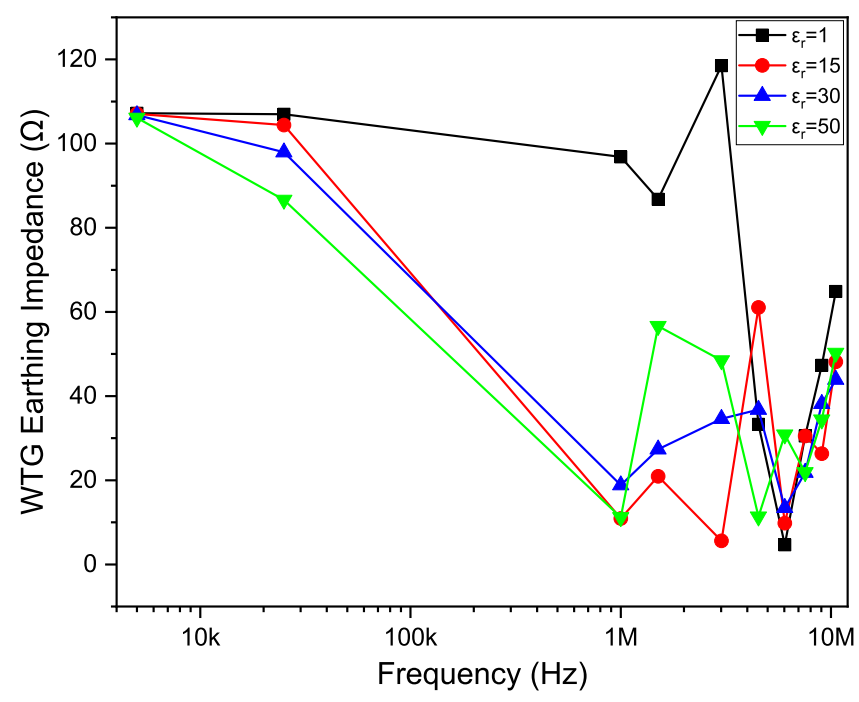

Figure 3.4: Frequency response of the WTG earthing impedance with change in relative permittivity with a constant soil resistivity $10 \mathrm{k} \Omega \mathrm{m}$.

\subsubsection{Effect of soil layer}

The effect of earth electrodes in different layers of soil stratification on the WTG impedance at $5 \mathrm{kHz}$ frequency is analysed in this section. For the soil model with a uniform soil resistivity, the WTG earthing impedance increases with an increase in the value of soil resistivity, as shown in Figure 3.6. For a two-layer soil structure, two different scenarios were studied. In each scenario, two soil resistivity values and two layer thickness 


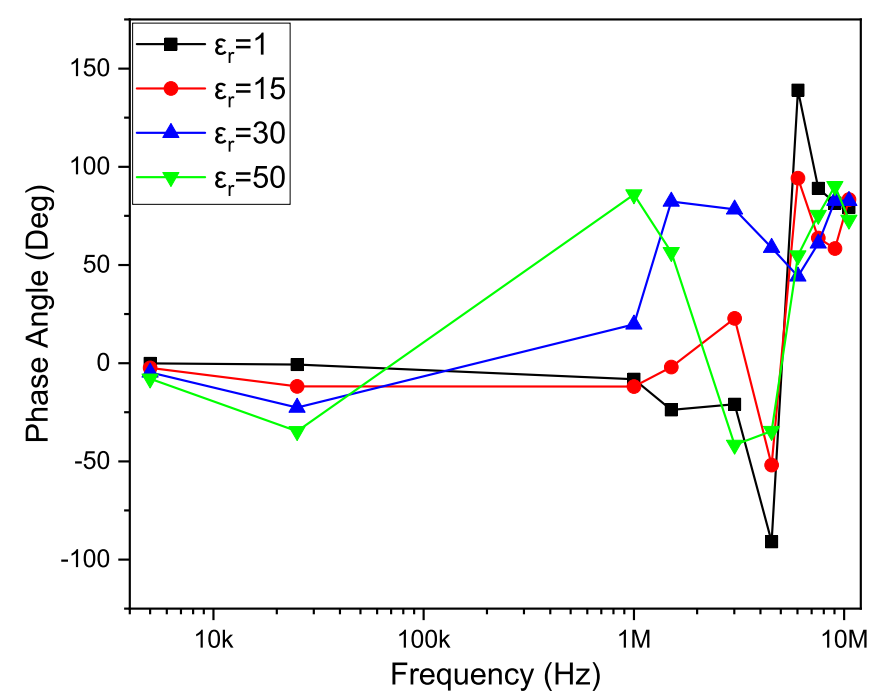

Figure 3.5: Frequency response of the phase angle with change in relative permittivity with a constant soil resistivity $10 \mathrm{k} \Omega \mathrm{m}$.

values are considered. For all of the cases, the earthing electrodes are buried at $1 \mathrm{~m}$ depth from the surface of the soil. The thickness of the layer is selected in such a way that the electrodes are placed in the top and bottom layers of the soil. Firstly, the soil resistivity of the top layer was kept constant, and the bottom layer resistivity was varied.

As depicted in Figure 3.6, with $100 \Omega \mathrm{m}$ top layer resistivity and earth electrodes in the top layer, the WTG earthing impedance increases with an increase in the bottom layer resistivity value up to $1000 \Omega \mathrm{m}$ and then it is constant with further increase in resistivity of the bottom layer. However, when the earth electrode is in the bottom layer, the impedance increases continuously with an increase in the resistivity of the bottom layer. A similar phenomenon is observed for the top layer soil whose resistivity is $1000 \Omega \mathrm{m}$.

In the next scenario, the resistivity of the bottom layer is maintained at a constant value, and the top layer resistivity is varied. It can be observed from Figure 3.7 that with a bottom layer resistivity $100 \Omega \mathrm{m}$ and the 


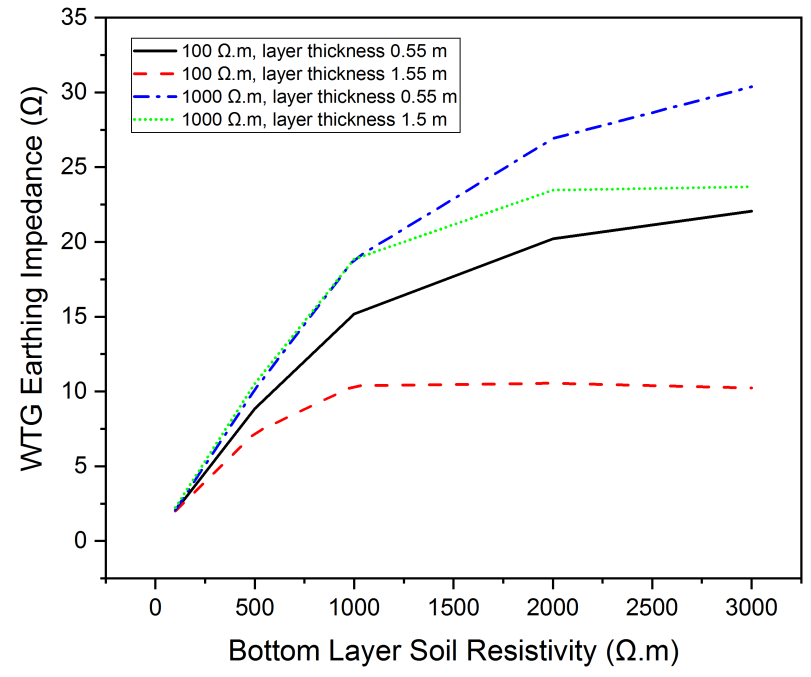

Figure 3.6: WTG earthing impedance at $5 \mathrm{kHz}$ frequency with constant resistivity of top layer. 
electrode in the top layer the WTG earthing impedance is constant with an increase in the top layer resistivity. When the earth electrode is in the bottom layer, the impedance is constant up to $1000 \Omega \mathrm{m}$ and then decreases below the resistivity values of the electrode in the top layer. An identical trend is noticed for the bottom layer resistivity of $1000 \Omega \mathrm{m}$.

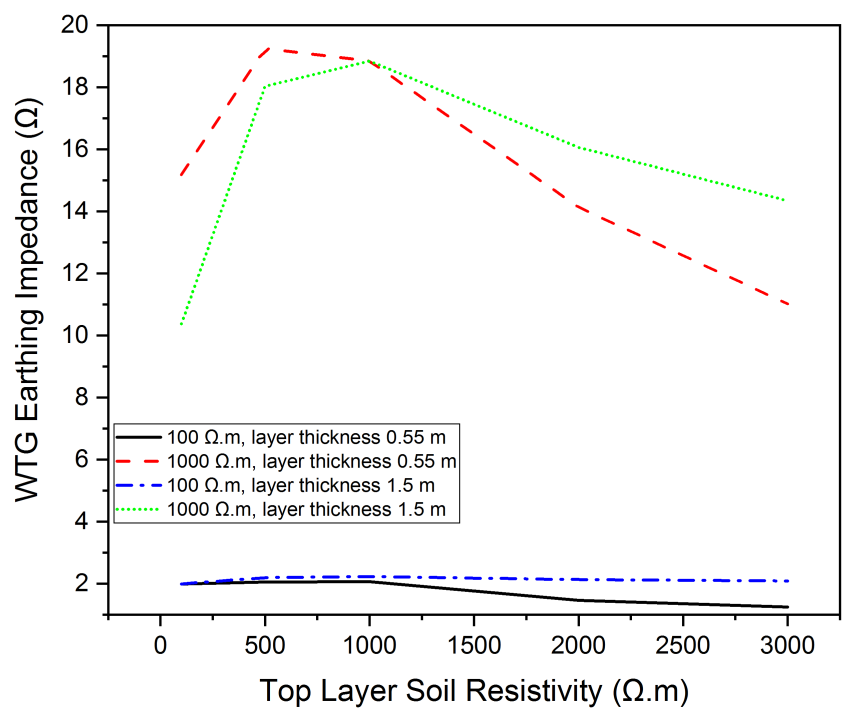

Figure 3.7: WTG earthing impedance at $5 \mathrm{kHz}$ frequency with constant resistivity of bottom layer.

This analysis shows that the uniform soil model results in higher earthing impedance compared to a horizontal multi-layer soil model irrespective of the earth electrode position in the soil layers. This is due to the lower soil resistivity of the second layer where the earth electrodes and most of the WTG rebar are installed. Moreover, it is the soil resistivity of the bottom layer which plays a predominant role in the overall earthing impedance due to the uniform distribution of electric fields. 


\subsubsection{Effect of frequency dependent soil parameters}

The WTG earthing impedance is strongly dependent on soil resistivity and relative permittivity of the soil. As discussed in Section 3.3, the soil resistivity and relative permittivity changes with frequency. Lightning discharge currents comprise high frequency-components, for which frequency dependency is significant in determining the impedance. Moreover, the variation in relative permittivity significantly affects the WTG earthing impedance.

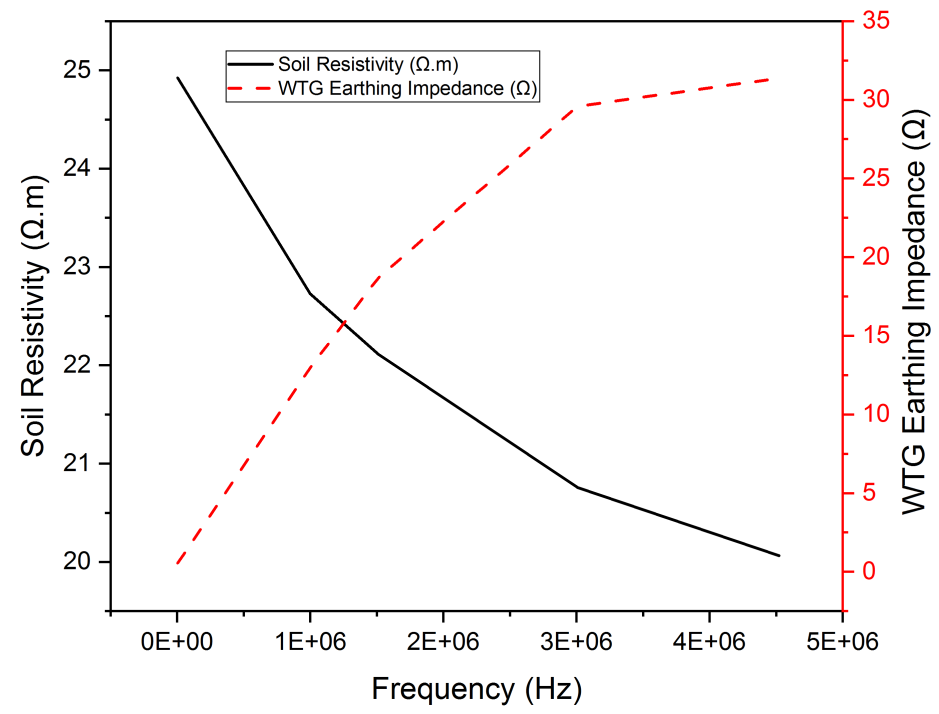

Figure 3.8: WTG earthing impedance with frequency dependent soil resistivity for $25 \Omega \mathrm{m}$.

Three different cases are considered in this analysis. In the first case, the relative permittivity is considered to be constant at all frequencies, and the soil resistivity is a frequency dependent value, calculated using Equation 3.7. Soil resistivity values of $25 \Omega \mathrm{m}, 250 \Omega \mathrm{m}$, and $2500 \Omega \mathrm{m}$ are considered. For $25 \Omega \mathrm{m}$, the impedance increases with frequency although there 
is a decrease in the soil resistivity with the increase in frequency, as depicted in Figure 3.8. This is due to the increase in both resistive and reactive components of ground potential with an increase in the frequency. In the case of $250 \Omega \mathrm{m}$ soil resistivity, the impedance increase to $3 \mathrm{MHz}$ and then decreases at $4.52 \mathrm{MHz}$, and again rises at $10.54 \mathrm{MHz}$. As depicted in Figure 3.9, for a soil resistivity of $2500 \Omega \mathrm{m}$, the WTG earthing impedance varies randomly with frequency. There is no common trend observed at higher soil resistivity values. The random behaviour is due to the change in soil resistivity, and the behaviour of the WTG earthing system at different frequencies. It is observed, the earthing system is resistive for lower frequencies and reactive (inductive or capacitive) for higher frequencies.

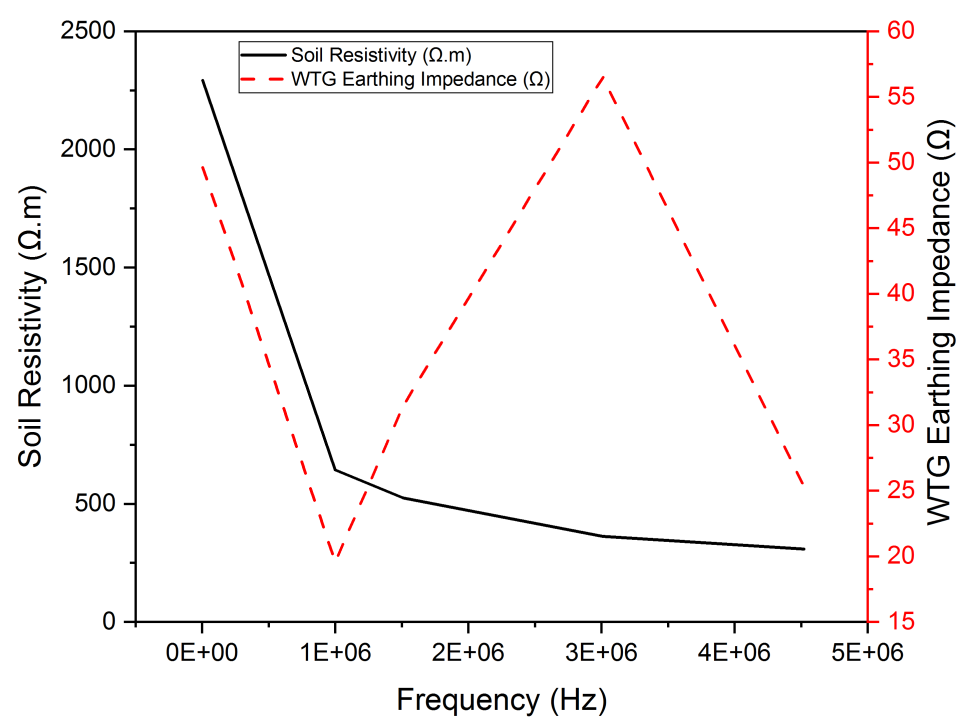

Figure 3.9: WTG earthing impedance with frequency dependent soil resistivity for $2500 \Omega \mathrm{m}$.

In the second scenario, the soil resistivity is considered to be independent of frequency, and the relative permittivity is a frequency dependent 
parameter, calculated using Equation 3.8. It is observed from Figure 3.10, that the relative permittivity has an influential role for the sites with high soil resistivity. The WTG earthing impedance at $250 \Omega \mathrm{m}$ is higher than $2500 \Omega \mathrm{m}$ after a frequency of $3 \mathrm{MHz}$. The decrease in relative permittivity with an increase in frequency, changes the earthing system behaviour, specially the capacitive reactance, which determines the earthing system behaviour at individual frequencies.

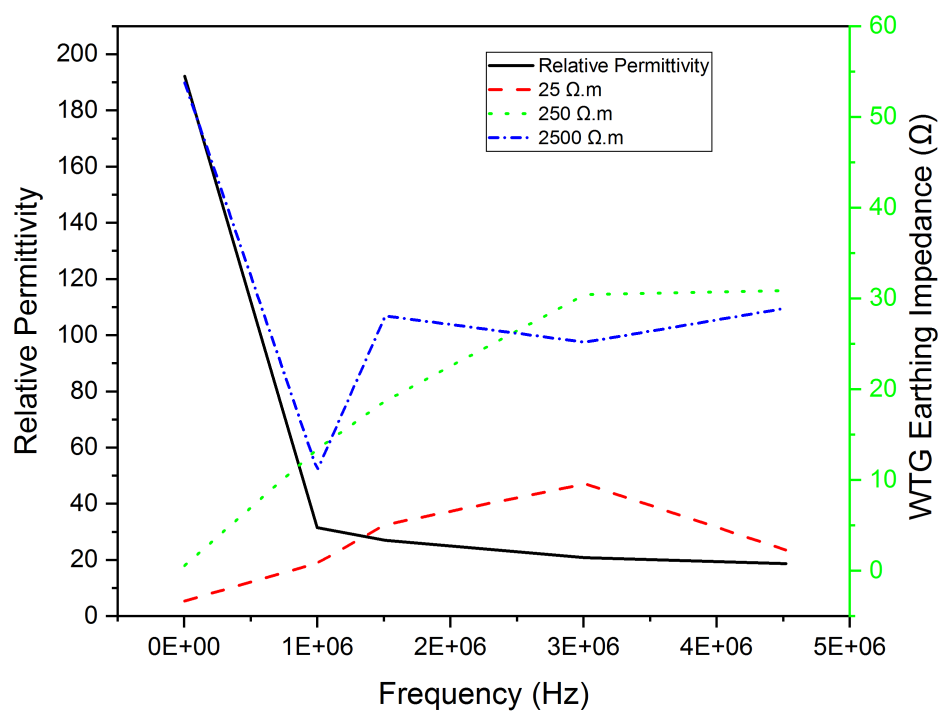

Figure 3.10: WTG earthing impedance with frequency dependent soil permittivity.

In the final case, both soil resistivity and relative permittivity are considered as frequency dependent parameters. From the results, it is observed that the change in WTG earthing impedance with frequency dependent soil parameters is significant at locations with high soil resistivity. From Figure 3.11, for an increase in frequency from $1 \mathrm{MHz}$ to $3 \mathrm{MHz}$, the increase in WTG earthing impedance is up to two times for $2500 \Omega \mathrm{m}$ soil 
resistivity. In the frequency range of $3.01 \mathrm{MHz}$ to $4.52 \mathrm{MHz}$ the impedance

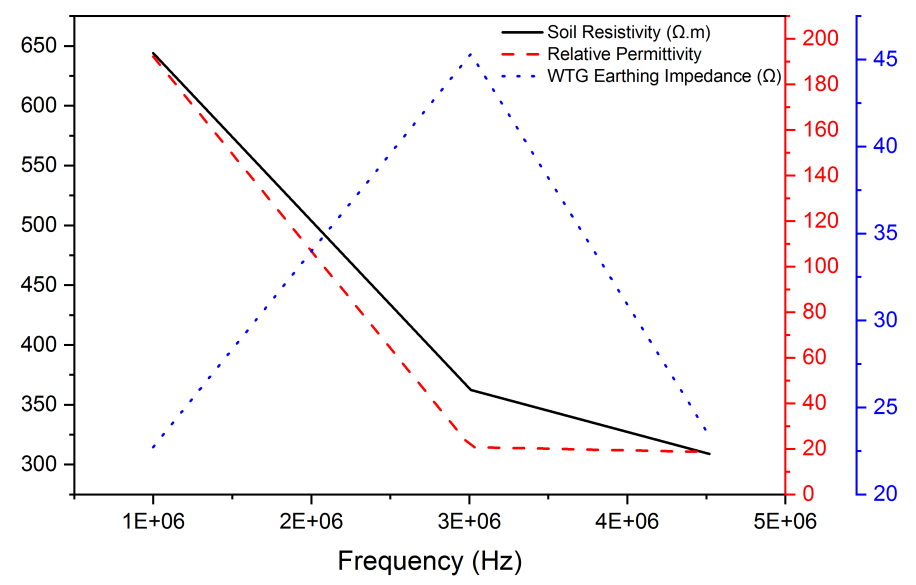

Figure 3.11: WTG earthing impedance with frequency dependent soil parameters for $2500 \Omega \mathrm{m}$.

halves. Again, the increase in impedance from $4.52 \mathrm{MHz}$ to $10.54 \mathrm{MHz}$ is more than twice the value. The variation of both soil resistivity and relative permittivity with frequency is the practical scenario. Hence, it is critical to consider the frequency dependent soil parameters for designing the WTG earthing system.

\subsubsection{WTG Impedance}

This section presents the comparison of frequency response of the WTG earthing impedance for three wind turbine locations at different scenarios. It also presents the comparison of the uniform, horizontal two-layer and three-layer soil structures, modelled in Table 3.3, are compared.

In the first case, a comparison of WTG earthing impedance with and without the connecting the rebar to the earthing system is performed. As 
depicted in Figures 3.12 to 3.14, connecting a rebar to the WTG earthing system reduces the impedance significantly regardless of the soil models used. For uniform soil resistivity, at a frequency of $5 \mathrm{kHz}$, the impedance reduces from $26.83 \Omega$ to $17.07 \Omega$, as shown in Fig 3.12. For the case of horizontal two-layer and three-layer, the WTG earthing impedance reduces from $10.8 \Omega$ to $9.12 \Omega$ and $10.06 \Omega$ to $8.74 \Omega$, respectively, as depicted in Fig 3.13 and 3.14. However, at higher frequencies, the reduction in impedance is more prominent and can be up to $76 \%$. The change in the impedance by connecting the rebar to the earthing system is a result of lightning down conduction current being shared by the metallic component of the rebar. However, a lower reduction of impedance for horizontal two-layer and three-layer structures at $5 \mathrm{kHz}$ is mainly due to the higher soil resistivity of the top layer.

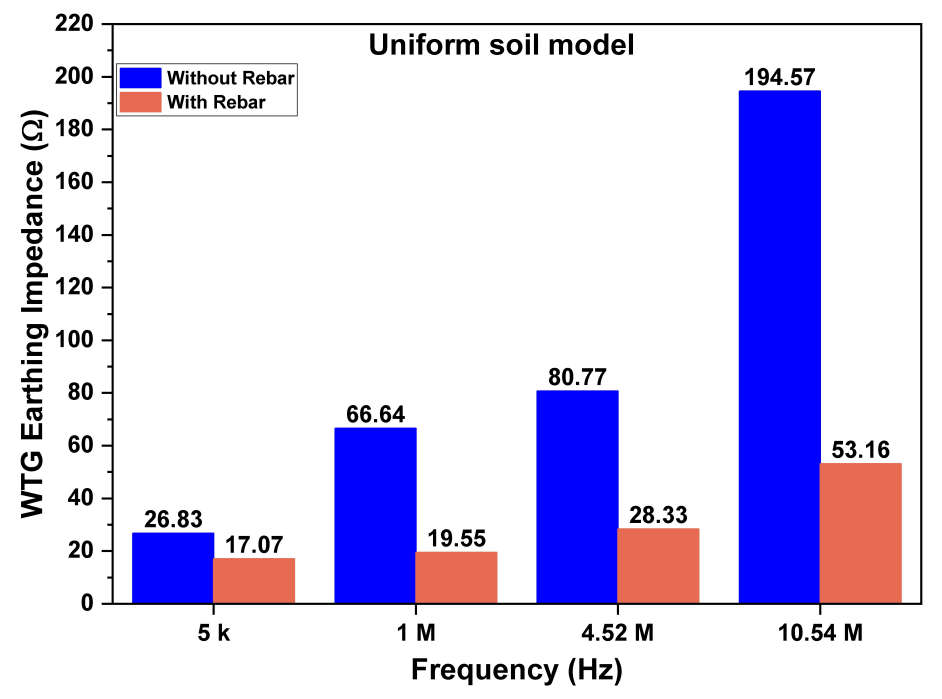

Figure 3.12: WTG earthing impedance of WTG-1-T1 with and without Rebar connected to earthing system for frequency independent soil parameters (Uniform soil model).

In the second scenario, the frequency response of WTG earthing 


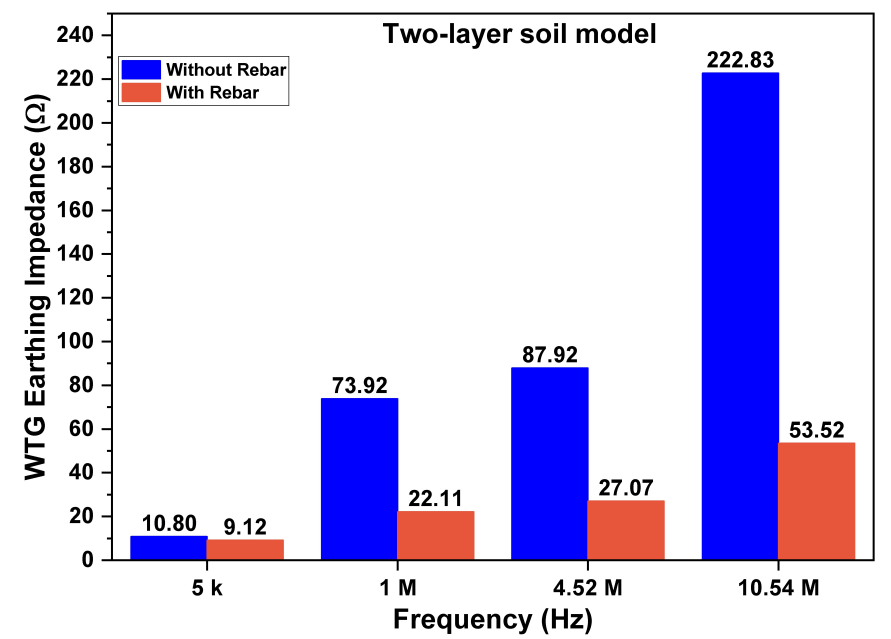

Figure 3.13: WTG earthing impedance of WTG-1-T1 with and without Rebar connected to earthing system for frequency independent soil parameters (Two-Layer soil model).

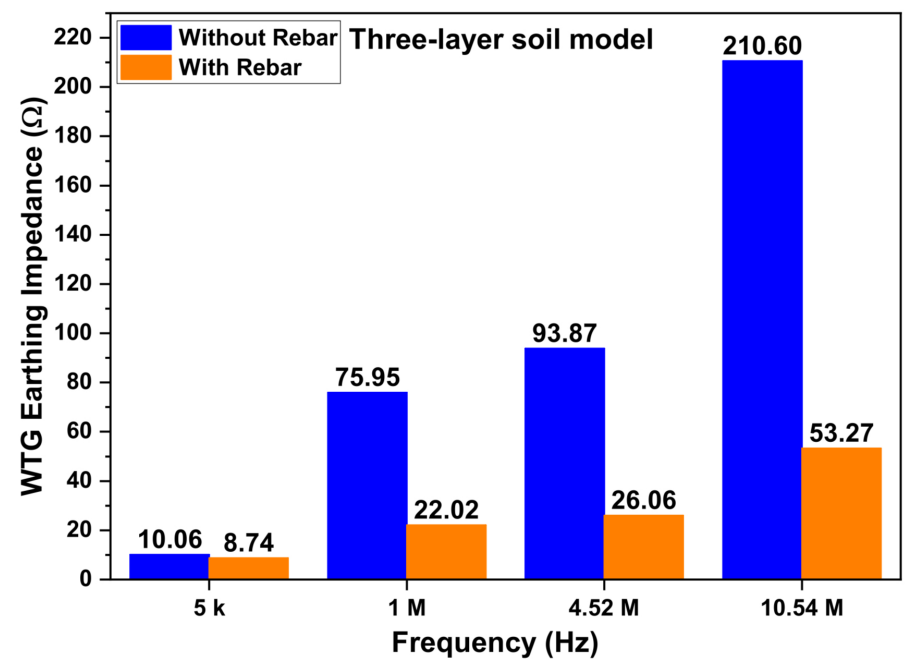

Figure 3.14: WTG earthing impedance of WTG-1-T1 with and without Rebar connected to earthing system for frequency independent soil parameters (Three-Layer soil model). 
impedance for frequency independent soil parameters with ring electrode connected to rebar is analysed for uniform, horizontal two-layer, and three-layer soil structures. As shown in Figure 3.15, for WTG-1-T1, a change from a uniform to a horizontal two-layer soil structure decreases the impedance from $19.06 \Omega$ to $16.69 \Omega$ at $5 \mathrm{kHz}$ frequency. In addition, the impedance is further reduced to $15.99 \Omega$ with a horizontal three-layer soil structure. However, at $1 \mathrm{MHz}$, a minimum increase in impedance from uniform to horizontal three-layer soil structure is observed. Again, at $4.52 \mathrm{MHz}$ the WTG earthing impedance decreases from uniform to horizontal three-layer soil models. Finally, at $10.54 \mathrm{MHz}$, the variation in impedance is negligible for different soil structures different structures of the soil.

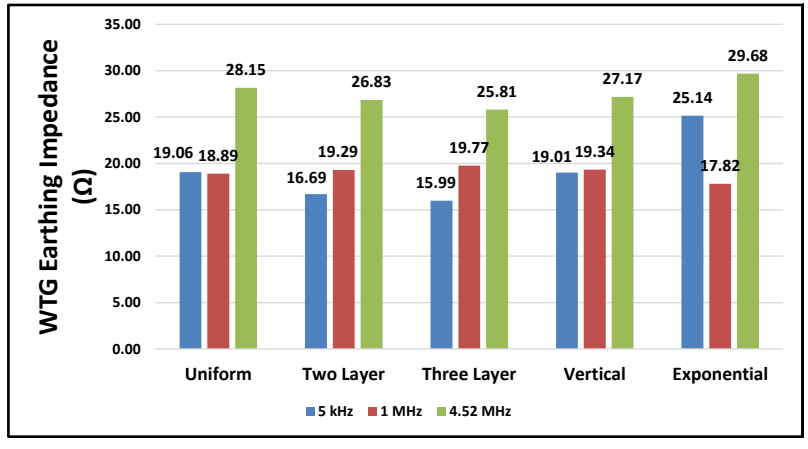

Figure 3.15: WTG earthing impedance of WTG-1-T1 with ring electrode for frequency independent soil parameters.

The change in impedance at different frequencies is mainly due to two components. Firstly, due to the change in the reactive component of the impedance. Secondly, due to the resistive component which increases significantly at higher frequencies. As illustrated in Figure 3.16, the phase angle is negligible at $5 \mathrm{kHz}$. However, at $1 \mathrm{MHz}$ a significant increase in phase angle leads to a higher reactive component. Moreover, the phase angle for the horizontal three-layer structure is more than that of the twolayer and uniform soil models resulting in a higher impedance value. At a 
frequency of $4.52 \mathrm{MHz}$, the phase angles drop significantly, even to negative values showing a capacitive reactive component. However, the resistive components have increased significantly leading to an overall increase in the WTG earthing impedance. Finally, at $10.54 \mathrm{MHz}$, due to a minimum variation of phase angle for different soil structures the change in WTG earthing impedance is negligible. Similar trends are observed for other wind turbine sites considered for this study.

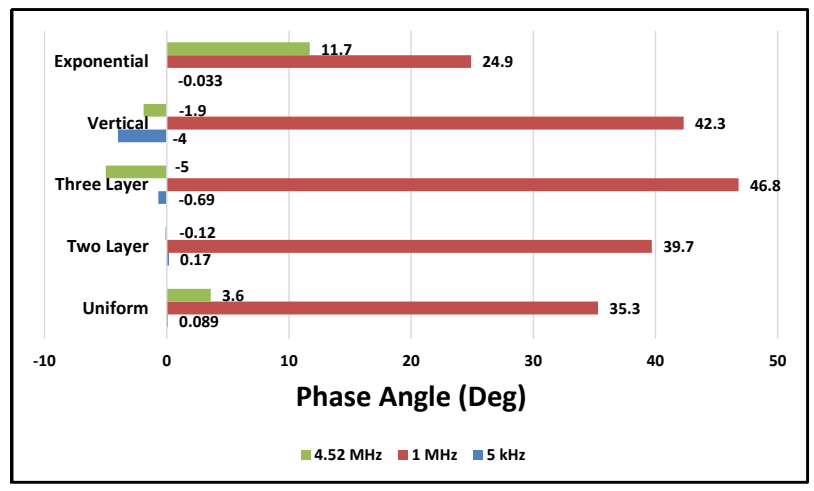

Figure 3.16: Phase angle of WTG-1-T1 with ring electrode for frequency independent soil parameters.

In the third case, a comparison of WTG earthing impedance with frequency independent and dependent soil parameters for all the wind turbine locations is performed. The frequency response of the WTG earthing impedance with frequency dependent soil parameters are analysed at three frequencies, $1 \mathrm{MHz}, 4.52 \mathrm{MHz}$ and $10.54 \mathrm{MHz}$, since the formula for frequency dependent soil parameters are not applicable for the frequencies below $10 \mathrm{kHz}$. For all the wind turbines, the frequency dependent soil parameters give the least impedance values compared to the frequency independent parameters regardless of soil model, as depicted in Figure 3.17. The reduction in WTG earthing impedance for frequency dependent soil parameters is mainly attributed to the significant reduction in soil resistivity at higher frequencies. Moreover, at some higher frequencies, the 
behaviour of the WTG earthing system is capacitive.

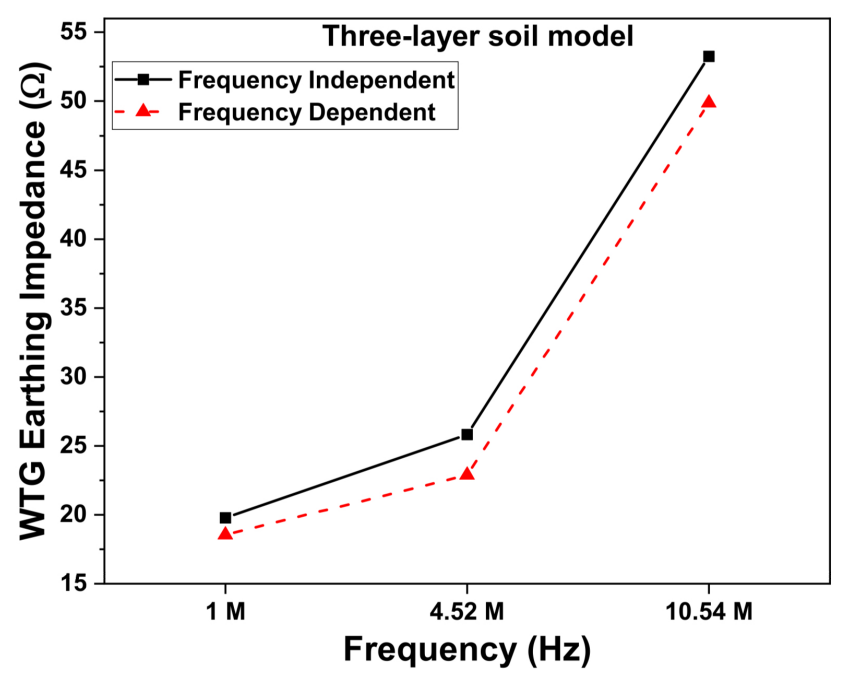

Figure 3.17: Comparison of WTG earthing impedance of WTG-1-T1 with ring electrode for frequency independent and dependent soil parameters.

In the fourth case, the horizontal earth electrodes are connected to the WTG earthing system and compared with the ring electrode alone earthing system. The frequency independent soil parameters are considered for this analysis. Figure 3.18 illustrates the effect of connecting horizontal electrodes for different soil structures at various frequencies. For uniform soil resistivity, the WTG earthing impedance reduces to $17.07 \Omega$ from $19.06 \Omega$ at $5 \mathrm{kHz}$. However, at $1 \mathrm{MHz}$ and $4.52 \mathrm{MHz}$, increase in the impedance is observed. At $10.54 \mathrm{MHz}$, the change in impedance with the addition of horizontal electrode is negligible. The reduction in impedance at low frequencies is a result of decreased resistance with horizontal electrode length. With increase in frequencies, the inductance and skin effect of the electrode increases the impedance value, which is evident at $1 \mathrm{MHz}$ and 4.52 MHz. The effect of electrode lengths after an effective length has no effect for high frequencies and hence no change in impedance, as observed 


\section{at $10.54 \mathrm{MHz}$.}

For horizontal two-layer soil structure, the reduction in impedance with horizontal electrodes is significant at $5 \mathrm{kHz}$, reducing from $16.69 \Omega$ to $9.12 \Omega$. Also, at the $5 \mathrm{kHz}$ frequency, the impedance decreases to $8.74 \Omega$ from $15.99 \Omega$ for horizontal three-layer soil structure. The ring electrode and horizontal electrodes are buried at a depth of $1 \mathrm{~m}$ from the surface of the earth which are in the second layer of the soil structure. The soil resistivity of the second layer is lower compared to the top layer. Also, the rebar structure is extended in the bottom layers of the soil, hence the overall earthing impedance is reduced. Similar effects are observed for all wind turbines with different values of soil resistivity.

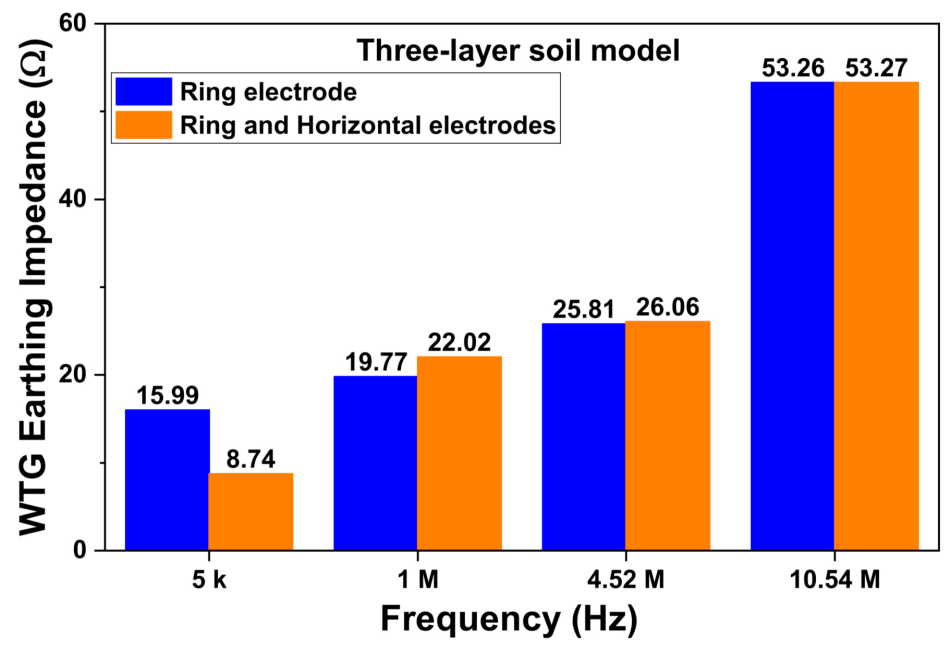

Figure 3.18: Comparison of WTG earthing impedance of WTG-1-T1 with ring electrode and ring \& horizontal electrodes for frequency independent soil parameters.

In the final case, the comparison of WTG earthing impedance with an earthing system comprising of ring \& horizontal electrodes for frequency independent and dependent soil parameters is performed. From Figure 3.19, it can be inferred that the frequency dependent soil parameters 


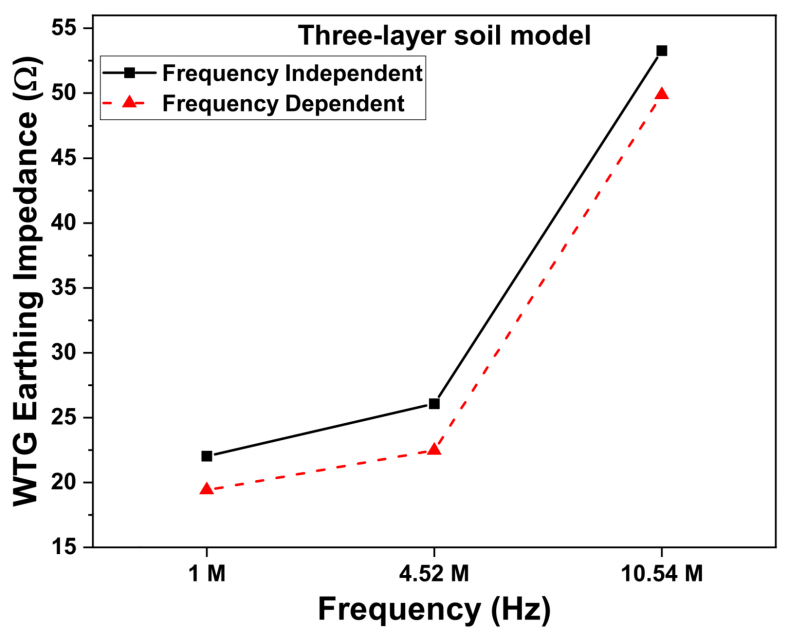

Figure 3.19: Comparison of WTG earthing impedance of WTG-1-T1 with ring \& horizontal electrodes for frequency independent and dependent soil parameters.

reduce the earthing impedance at all frequencies for all the soil structures due to the reduced soil resistivity with an increase in frequency. However, it is observed that the reduction is maximum for the horizontal three-layer soil structure. It is important to note that the WTG earthing impedance with the horizontal electrodes is lower than that with a ring electrode alone for both frequency independent and dependent soil parameters at low frequencies. The increased impedances are a result of the inductive reactance of the electrodes.

\subsubsection{Ground Potential Rise}

The ground potential rise profile of the WTG earthing system with only ring electrode scenario, without horizontal and vertical electrodes) for WTG-1-T1 under uniform soil structure at a frequency of $5 \mathrm{kHz}$ is observed to be equipotential, as shown in Figure 3.20 with a peak poten- 
tial rise of about $952 \mathrm{kV}$, as depicted in Figure 3.21. The potential value drops by $4 \%$ at the periphery of the WTG earthing foundation. The potential is reduced by $36.5 \%$ within a few meters from the WTG foundation. For the horizontal two-layer soil structure a similar equipotential profile is observed with a reduction of $12.4 \%$. The potential rise is further reduced by $16 \%$ with the horizontal three-layer soil structure. The reduction in potential, a few meters away from the WTG foundation, is due to the concentrated electric fields and the current density at the WTG earthing electrodes.

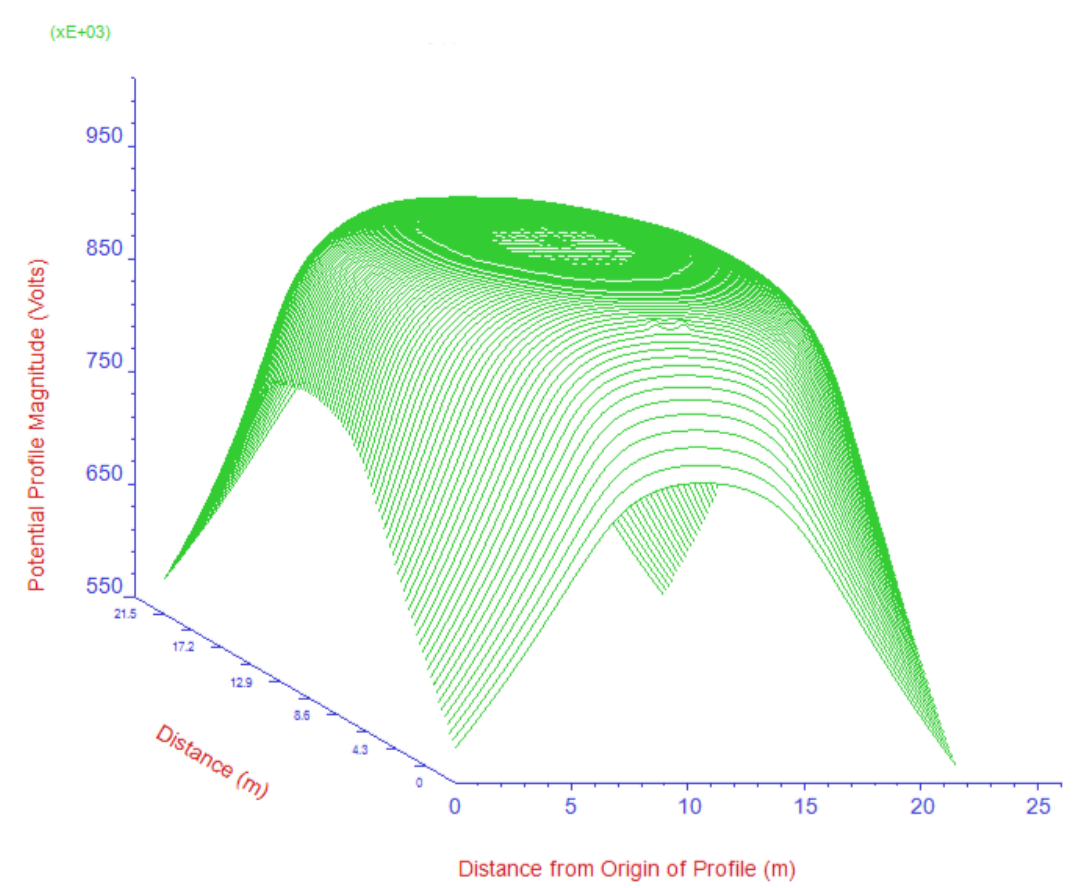

Figure 3.20: Ground potential rise for WTG-1-T1 with three-layer soil resistivity with electrodes at $5 \mathrm{kHz}$.

In the next scenario, the GPR of the earthing system consisting of a combination of ring and horizontal electrodes is analysed. The potential profile exhibits an equipotential nature for all the soil models. However, 


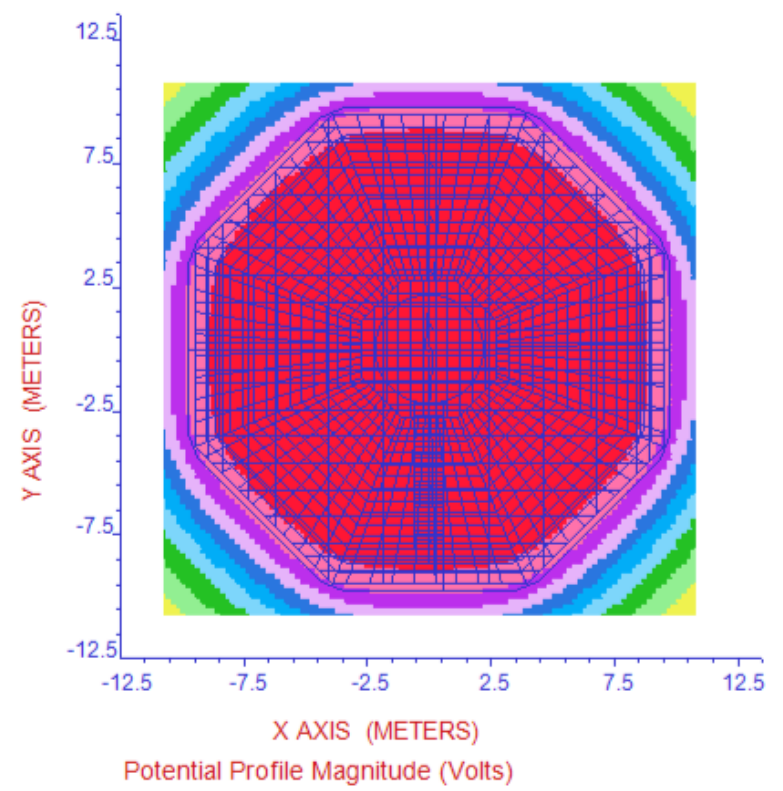

\begin{tabular}{|c|c|c|}
\hline \multicolumn{3}{|c|}{ SPOT LEVELS $\times 1 . E+3$} \\
\hline Maximum & Value: & 952.705 \\
\hline Minimum & Value : & 565.980 \\
\hline 6 & 952.71 & \\
\hline 6 & 914.03 & \\
\hline 6 & 875.36 & \\
\hline 6 & 836.69 & \\
\hline 6 & 798.02 & \\
\hline 6 & 759.34 & \\
\hline 6 & 720.67 & \\
\hline 6 & 682.00 & \\
\hline 6 & 643.32 & \\
\hline 6 & 604.65 & \\
\hline
\end{tabular}

Figure 3.21: Ground potential rise for WTG-1-T1 with three-layer soil resistivity with ring electrodes at $5 \mathrm{kHz}$. 
the peak value of ground potential is reduced for all the soil structures when compared to ring electrode alone scenario. For the uniform soil structure, the peak potential reduces by $10.4 \%$. For the two-layer soil structure, the peak potential reduction is $45.3 \%$ and $47.6 \%$ for the threelayer soil structure (Figure 3.22). The reduction in GPR is mainly due to uniform electric field distribution at the earthing system.

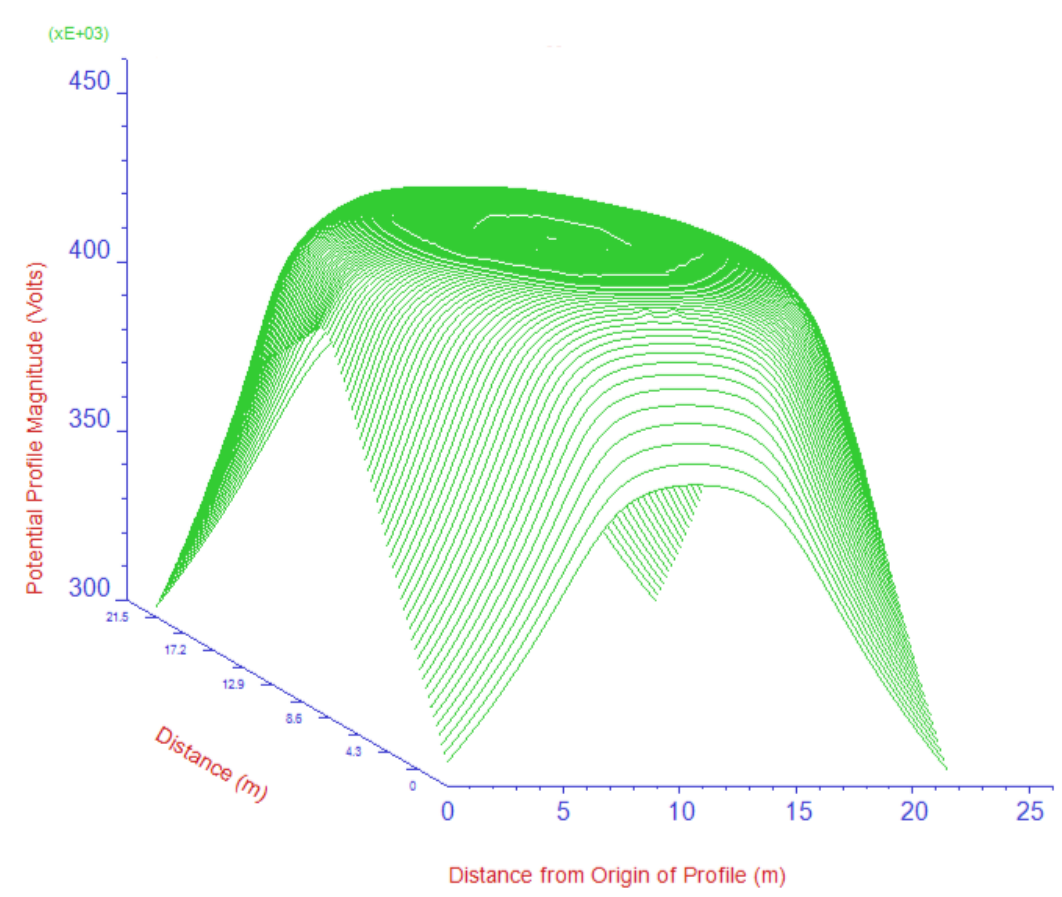

Figure 3.22: Ground potential rise for WTG-1-T1 with three-layer soil resistivity with ring and horizontal electrodes at $5 \mathrm{kHz}$.

In the third scenario, the potential profile at a higher frequency of $1 \mathrm{MHz}$ is analysed. For the uniform soil structure, the potential profile is different from that of $5 \mathrm{kHz}$, with high potential spikes at the horizontal electrode connection, with a peak of about $1569 \mathrm{kV}$, as depicted in Figure 3.24 .

There is a reduced potential where the WTG connects to the horizontal 


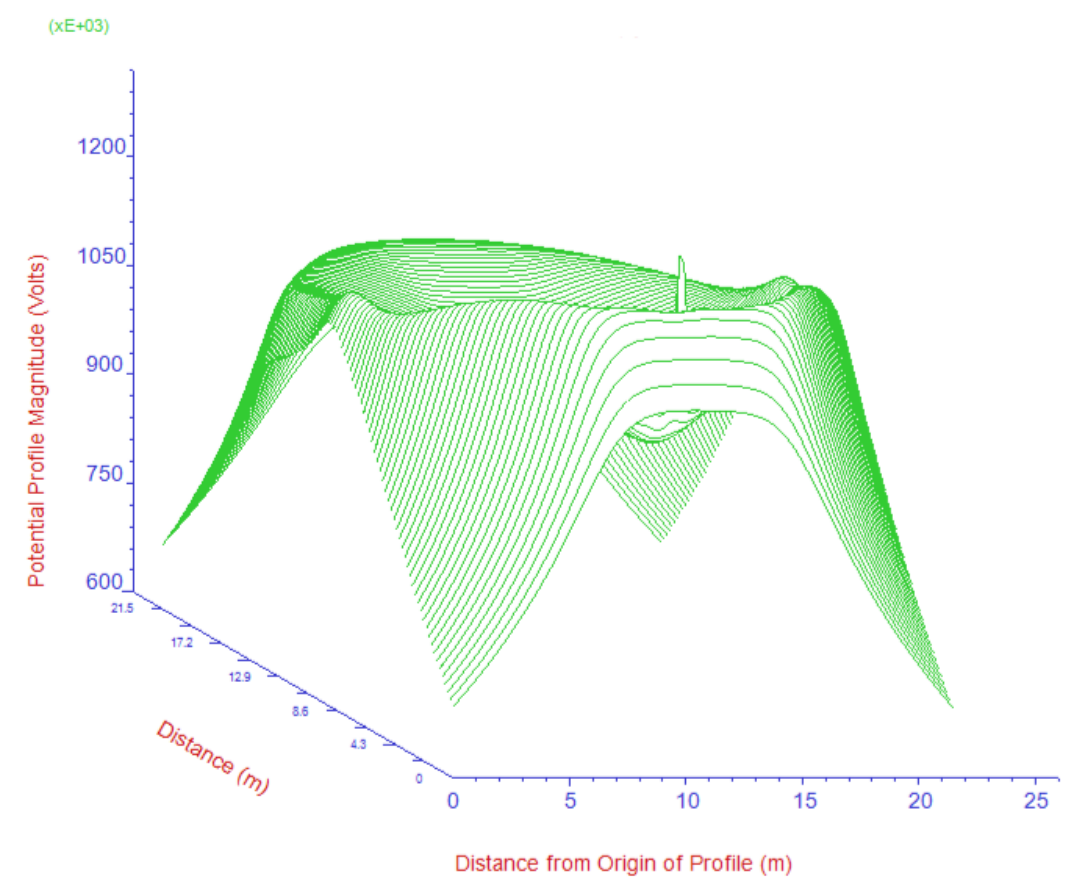

Figure 3.23: Ground potential rise for WTG-1-T1 with three-layer soil resistivity with ring and horizontal electrodes at $1 \mathrm{MHz}$. 

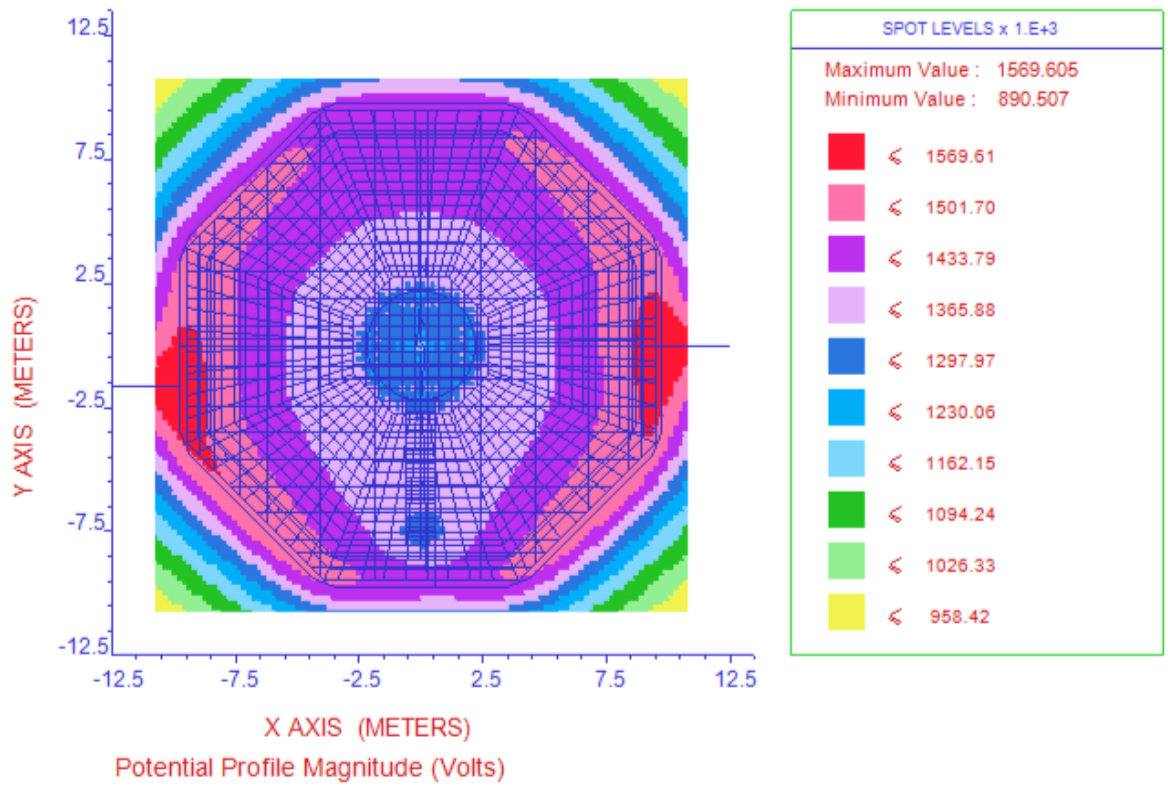

Potential Profile Magnitude (Volts)

Figure 3.24: Ground potential rise for WTG-1-T1 with three-layer soil resistivity with ring and horizontal electrodes at $1 \mathrm{MHz}$. 
electrode. A similar profile is observed for horizontal two-layer soil structure with a reduced peak value. However, a different potential profile is observed for the three-layer soil structure with a more uniform potential with local maxima, as depicted in Figure 3.23. The non-uniform potential distribution at higher frequencies is due to higher current dissipation at the point of impulse injection and electrode connection points. It is observed that the earthing system with a combination of ring and horizontal electrodes reduces the potential rise significantly in comparison to other electrode configurations.

\subsection{Chapter summary}

This chapter discussed in detail the analysis of wind turbine earthing impedance considering several factors. Firstly, the analysis of soil modelling of the measured soil resistivity values is performed. Secondly, the effect of frequency dependent soil parameters on the WTG earthing impedance is quantified. Next, the effects of rebar, horizontal electrodes, soil structures and frequency dependent soil parameters on the WTG earthing impedance are evaluated. Also, the impact of earth electrodes in various soil layers is carried out. Finally, the ground potential rise is evaluated. The main contributions of this chapter are:

1. Based on the analysis, the horizontal three-layer structure is the optimum soil structure with the lowest RMS error.

2. Frequency dependency of soil parameters is critical in wind turbine generator earthing design since the variation in earthing impedance is significant with frequency dependent soil parameters.

3. The inclusion of rebar in the earthing design has a significant diminishing effect on the grounding impedance. This conclusion is based on full wave electromagnetic models that considered the complete 
foundation structure, which has not previously been reported in the literature.

4. The modelled wind turbine earthing impedance is lowest for a horizontal three-layer soil model at low frequencies.

5. The WTG earthing impedance with frequency dependent soil parameters is lesser than that with the frequency independent soil parameters.

6. At high frequencies, the horizontal electrodes have a negligible role in determining the WTG earthing impedance.

7. The bottom layer soil resistivity plays a significant role in determining the WTG earthing impedance.

8. The potential distribution is uniform at lower frequencies. The electrode combination of ring and horizontal electrodes reduces the peak potential values.

These conclusions will provide information for the future design of WTG grounding systems to facilitate the continued worldwide adoption of WTGs as a source of clean and sustainable power. 


\section{Chapter 4}

\section{Effects of Direct Lightning Strikes on Wind Turbine Generators}

The WTG earthing system should be capable of protecting the wind turbine from various lightning discharge parameters it experiences when struck by lightning. The lightning parameters include frequency, peak current, charge transfer, steepness, and rise time and fall time of the lightning discharge current waveforms and varies with the lightning protection level and the type of lightning strikes. Hence, it is important to identify the influencing parameters of the lightning discharge current on the WTG earthing system.

This chapter presents an analysis of the wind turbine earthing system for a horizontal multi-layer soil model in the event of a direct lightning strike on the wind turbine generator for various lightning discharge current waveforms. The analysis of the frequency components and peak current magnitudes of different lightning current waveforms and their impact on the earthing system of the wind turbine is performed. 


\subsection{Description of the model}

The wind turbine earthing system is designed by connecting the WTG foundation to the earth electrodes. Figure 4.1 depicts the perspective three-dimensional (3-D) view of the wind turbine foundation and the WTG earthing system. The electrode lengths are determined by the soil resistivity of the selected WTG site. The maximum resistivity value of the soil is used for determining the electrode lengths for this analysis.

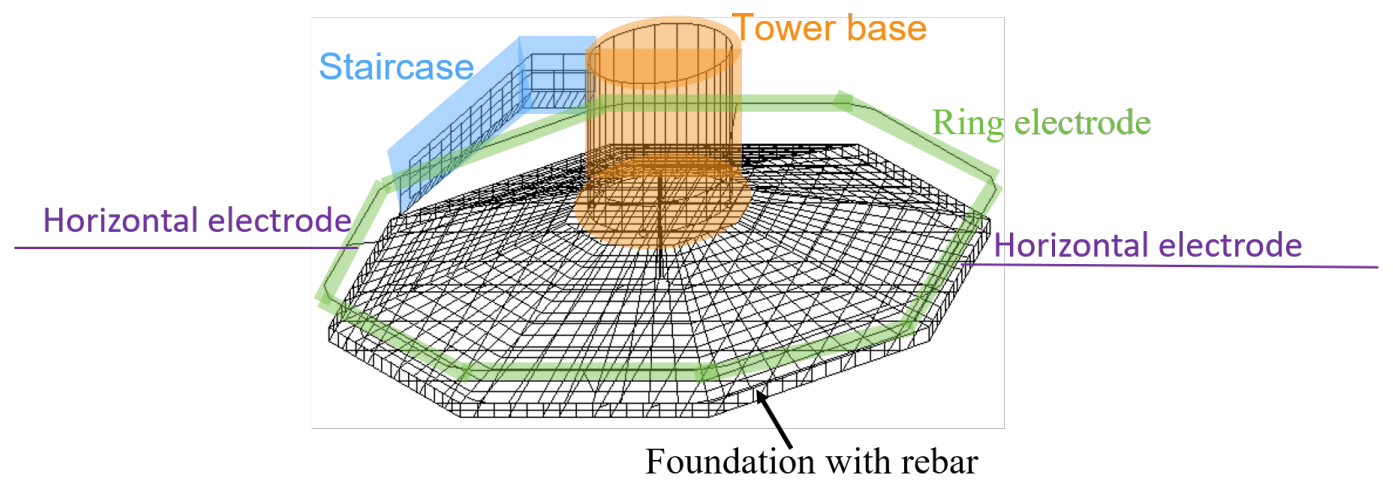

Figure 4.1: WTG earthing system.

Soil structure is modelled as a horizontal multi-layer with the resistivity varying vertically through the soil. The details of soil resistivity measurement and modelling are presented in Section 3.2.

A realistic evaluation of the effects of a lightning strike on a wind turbine depends on the proper representation of lightning discharge currents [66]. According to IEC 61400-24 [17], three lightning discharge current waveforms have been used in this analysis. Figure 4.2 shows the first short positive stroke with a rise time of $10 \mu \mathrm{s}$, for a time to half value $350 \mu \mathrm{s}$ 
and the peak current of $200 \mathrm{kA}$. Similarly, the corresponding first short negative stroke and a subsequent short stroke, for a wave shape and a peak current considered for research are $1 / 200 \mu \mathrm{s}, 100 \mathrm{kA}$ and $0.25 / 100 \mu \mathrm{s}$, $50 \mathrm{kA}$, as depicted in Figures 4.3 and 4.4, respectively.

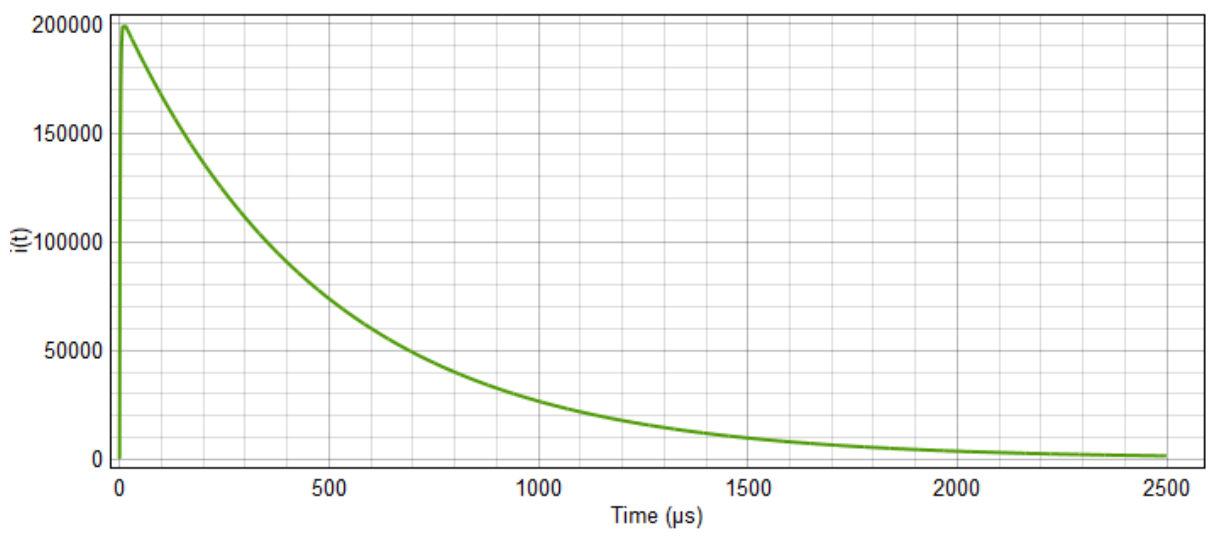

Figure 4.2: Lightning current waveform of the first short positive stroke.

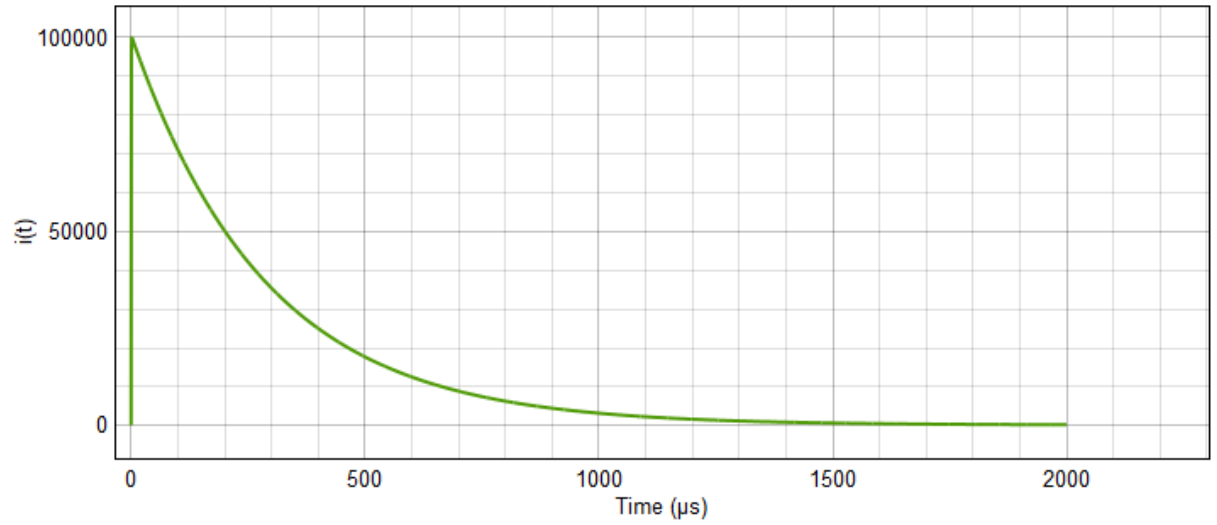

Figure 4.3: Lightning current waveform of the first short negative stroke.

This study is based on the first short positive stroke, the first short negative stroke and a subsequent short stroke with rise and fall times of $10 / 350 \mu \mathrm{s}, 1 / 200 \mu \mathrm{s}$, and $0.25 / 100 \mu \mathrm{s}$, respectively, with lightning protec- 


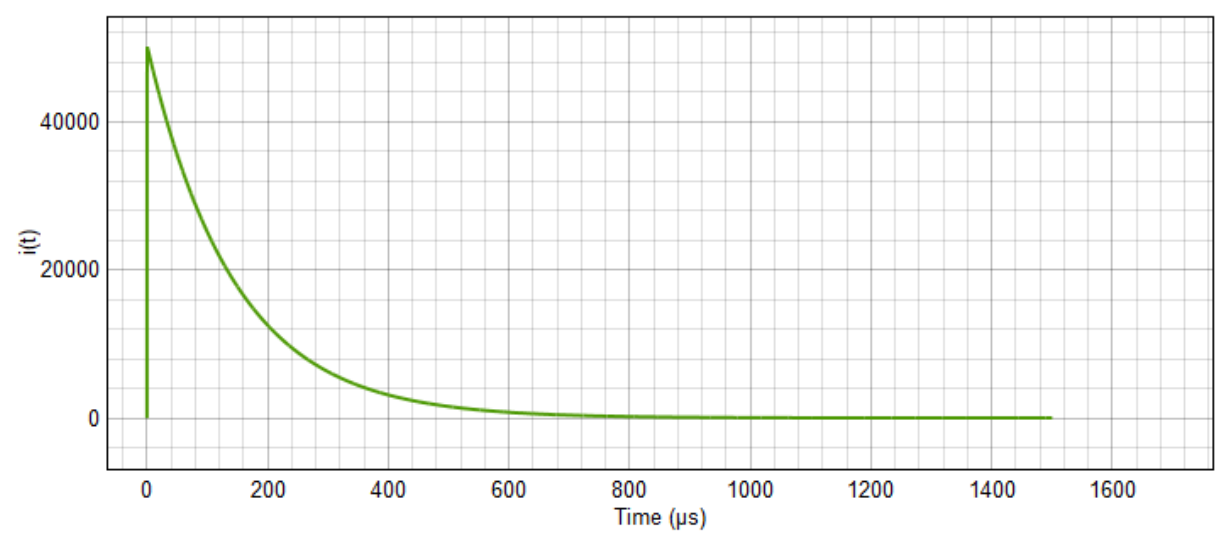

Figure 4.4: Lightning current waveform of a subsequent short stroke.

tion level-1. The rationale for choosing these parameters is that the design of the LPS of a wind turbine is based on these lightning parameters [17].

\subsection{Results and Discussion}

The detailed measurements and modelling of the resistivity of the soil are described in Chapter 3. From the modelled soil structures, two wind turbine sites with low and high soil resistivity are considered for this analysis. The Wenner method [173] is used for measuring the soil resistivity at the wind turbine sites. The measured apparent resistivity data is modelled as a horizontal three-layer model with corresponding resistivity values depicted in Table 4.1: $\rho_{1}, \rho_{2}$ and $\rho_{3}$ are the top, central and bottom layer resistivites, respectively.

Table 4.1: Soil Resistivity Values of Wind Turbine Locations

\begin{tabular}{|c|c|c|c|c|c|c|}
\hline Location & $\begin{array}{c}\rho_{1} \\
{[\Omega \mathrm{m}]}\end{array}$ & $\begin{array}{c}\text { Thickness } \\
{[\mathrm{m}]}\end{array}$ & $\begin{array}{c}\rho_{2} \\
{[\Omega \mathrm{m}]}\end{array}$ & $\begin{array}{c}\text { Thickness } \\
{[\mathrm{m}]}\end{array}$ & $\begin{array}{c}\rho_{3} \\
{[\Omega \mathrm{m}]}\end{array}$ & $\begin{array}{c}\text { Thickness } \\
{[\mathrm{m}]}\end{array}$ \\
\hline 1 & 85 & 0.4 & 39 & 21 & 74 & $\infty$ \\
\hline 2 & 1710 & 2.2 & 806 & 13 & 1762 & $\infty$ \\
\hline
\end{tabular}


The frequency response of the WTG earthing system are calculated for three lightning discharge current wave shapes at two wind turbine sites. WTG-1 in this work represent the low soil resistivity wind turbine site and WTG-2 represents the higher soil resistivity site. The earthing system of WTG-1 consists of a ring earth electrode, the wind turbine foundation and the other metallic components of the WTG. As the soil resistivity of all the three layers is less than $500 \Omega \mathrm{m}$, an earth electrode length of $5 \mathrm{~m}$ is the required minimum electrode length adhering to the standard IEC 6140024 [17]. However, for WTG-2, the maximum soil resistivity is $1762 \Omega \mathrm{m}$ for the bottom layer, hence an electrode length of $45 \mathrm{~m}$ has been selected. Hence, the earthing system for WTG-2 comprises of a ring earth electrode, two horizontal electrodes, the WTG foundation and other metallic components.

The important assumptions made for this analysis are: lightning strikes at the bottom of the WTG tower and the earth electrode lengths are calculated according to the maximum resistivity value of a three-layer soil model: this corresponds to the radius of the ring electrode length of $10 \mathrm{~m}$ and the remaining length of the electrode are compensated by two horizontal electrodes each having a length of $\left(l_{1}\right)-10$. Here $l_{1}$ is the minimum required length of the earth electrode. The steel tubular tower of the WTG is conductive and can conduct the lightning discharge current to the grounding network and hence the tower is neglected for this analysis.

The time domain lightning discharge current waveforms are transformed into the frequency domain, using the FFTSES module available of the CDEGS software package, are illustrated in Table 4.2. The frequency spectrum suggests several dominant frequencies for each of the time domain lightning current waveforms. The value of the low-frequency considered for the purpose of this analysis for all the lightning current wave shapes is $5 \mathrm{kHz}$, which represents the low to steady state values. The high frequencies vary with the rise time, between $400 \mathrm{kHz}$ and $5.46 \mathrm{MHz}$, based on the peak value of the current magnitude in the time domain waveform. 
For the first short positive stroke waveform, the high frequency considered is $410 \mathrm{kHz}$. The value is $1 \mathrm{MHz}$ for first short negative stroke and $5.46 \mathrm{MHz}$ for the subsequent stroke.

\subsubsection{WTG Impedance}

The frequency response of the WTG earthing impedance for the first short positive stroke with a peak current magnitude of $200 \mathrm{kA}$ is depicted in Figure 4.5. The grounding impedance increases from $0.85 \Omega$ at $5.2 \mathrm{kHz}$ to $7.17 \Omega$ at $410 \mathrm{kHz}$ for WTG-1. A dominant increase in the inductive component results in an increased earthing impedance, demonstrated by an increase in phase angle from $5.1^{\circ}$ to $72.6^{\circ}$. However, for WTG-2, the earthing impedance decreases with frequency from $17.54 \Omega$ at $5.2 \mathrm{kHz}$ to $15.42 \Omega$ at $410 \mathrm{kHz}$, as shown in Figure 4.5. This is due to the decrease in inductive components for high soil resistivity values.

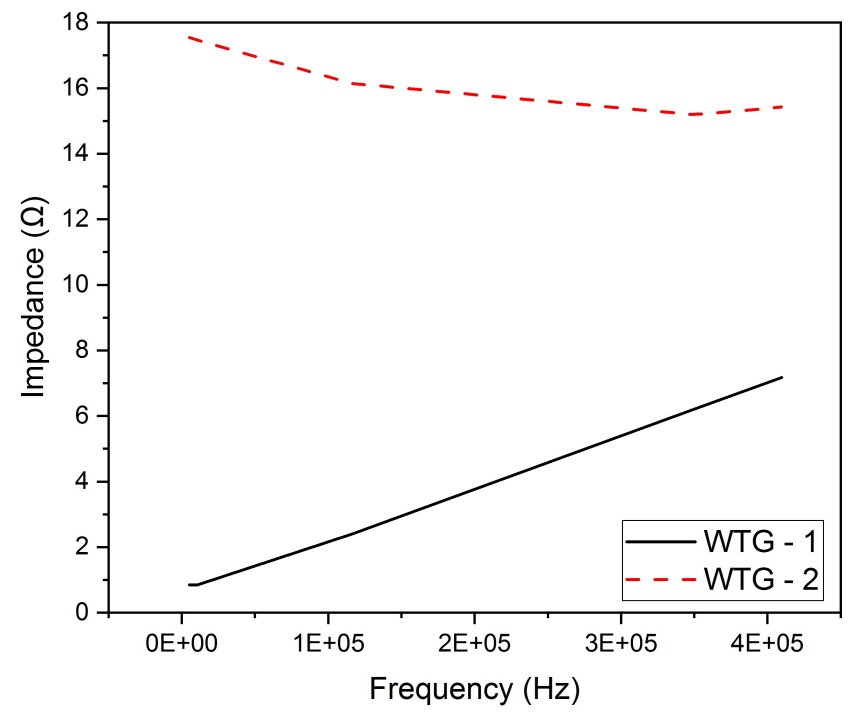

Figure 4.5: WTG earthing impedance for the first short positive stroke lightning current parameters.

The wind turbine grounding impedance for the first short negative 
Table 4.2: Dominant Frequencies and Corresponding Amplitude for Different Lightning Strokes

\begin{tabular}{|c|c|c|c|c|c|c|}
\hline \multirow[t]{2}{*}{ S.No. } & \multicolumn{2}{|c|}{ First short positve stroke } & \multicolumn{2}{|c|}{ First short negative stroke } & \multicolumn{2}{|c|}{ Subsequent short stroke } \\
\hline & $\begin{array}{c}\text { Frequency } \\
(\mathrm{Hz})\end{array}$ & $\begin{array}{c}\text { Amplitude } \\
(\mathrm{kA})\end{array}$ & $\begin{array}{c}\text { Frequency } \\
(\mathrm{Hz})\end{array}$ & $\begin{array}{c}\text { Amplitude } \\
(\mathrm{kA})\end{array}$ & $\begin{array}{c}\text { Frequency } \\
(\mathrm{Hz})\end{array}$ & $\begin{array}{c}\text { Amplitude } \\
\text { (kA) }\end{array}$ \\
\hline 1 & $5.00 \mathrm{E}+01$ & $3.24208 \mathrm{E}-16$ & $5.00 \mathrm{E}+01$ & $5.34136 \mathrm{E}-29$ & $5.00 \mathrm{E}+01$ & $2.1138 \mathrm{E}-59$ \\
\hline 2 & $4.00 \mathrm{E}+02$ & 1.220186513 & $5.00 \mathrm{E}+02$ & 0.094255359 & $6.67 \mathrm{E}+02$ & 0.001484942 \\
\hline 3 & $8.00 \mathrm{E}+02$ & 15.81136092 & $1.00 \mathrm{E}+03$ & 3.076161044 & $1.33 \mathrm{E}+03$ & 0.272743656 \\
\hline 4 & $1.20 \mathrm{E}+03$ & 37.13786467 & $1.50 \mathrm{E}+03$ & 9.830525635 & $2.00 \mathrm{E}+03$ & 1.550403343 \\
\hline 5 & $1.60 \mathrm{E}+03$ & 56.91683839 & $2.00 \mathrm{E}+03$ & 17.57359349 & $2.67 \mathrm{E}+03$ & 3.696479486 \\
\hline 6 & $2.00 \mathrm{E}+03$ & 73.53516498 & $2.50 \mathrm{E}+03$ & 24.90180785 & $3.33 \mathrm{E}+03$ & 6.225744497 \\
\hline 7 & $2.40 \mathrm{E}+03$ & 87.22974574 & $3.00 \mathrm{E}+03$ & 31.41553153 & $4.00 \mathrm{E}+03$ & 8.813107644 \\
\hline 8 & $2.80 \mathrm{E}+03$ & 98.54687254 & $3.50 \mathrm{E}+03$ & 37.08732483 & $4.67 \mathrm{E}+03$ & 11.29643976 \\
\hline 9 & $3.20 \mathrm{E}+03$ & 107.9882556 & $4.00 \mathrm{E}+03$ & 42.0035822 & $5.33 \mathrm{E}+03$ & 13.6081728 \\
\hline 10 & $3.60 \mathrm{E}+03$ & 115.9526021 & $4.50 \mathrm{E}+03$ & 46.27363707 & $6.00 \mathrm{E}+03$ & 15.72861689 \\
\hline 11 & $4.00 \mathrm{E}+03$ & 122.7449625 & $5.00 \mathrm{E}+03$ & 50.00016999 & $6.67 \mathrm{E}+03$ & 17.66050234 \\
\hline 12 & $4.40 \mathrm{E}+03$ & 128.5972651 & $5.50 \mathrm{E}+03$ & 53.2713072 & $8.00 \mathrm{E}+03$ & 21.01218733 \\
\hline 13 & $4.80 \mathrm{E}+03$ & 133.6867745 & $6.00 \mathrm{E}+03$ & 56.16014857 & $8.67 \mathrm{E}+03$ & 22.46454983 \\
\hline 14 & $5.20 \mathrm{E}+03$ & 138.1502952 & $6.50 \mathrm{E}+03$ & 58.72657824 & $9.33 \mathrm{E}+03$ & 23.78905418 \\
\hline 15 & $5.60 \mathrm{E}+03$ & 142.0945971 & $7.00 \mathrm{E}+03$ & 61.01952127 & $1.07 \mathrm{E}+04$ & 26.11004953 \\
\hline 16 & $6.40 \mathrm{E}+03$ & 148.7456949 & $8.50 \mathrm{E}+03$ & 66.6237964 & $7.80 \mathrm{E}+05$ & 49.65274659 \\
\hline 17 & $7.20 \mathrm{E}+03$ & 154.1332697 & $1.05 \mathrm{E}+04$ & 72.0360502 & $1.02 \mathrm{E}+06$ & 49.75807121 \\
\hline 18 & $8.00 \mathrm{E}+03$ & 158.5834934 & $1.35 \mathrm{E}+04$ & 77.55073794 & $1.19 \mathrm{E}+06$ & 49.8065678 \\
\hline 19 & $9.20 \mathrm{E}+03$ & 163.9718934 & $7.35 \mathrm{E}+04$ & 95.74530461 & $1.37 \mathrm{E}+06$ & 49.84305189 \\
\hline 20 & $1.08 \mathrm{E}+04$ & 169.4734571 & $1.46 \mathrm{E}+05$ & 98.02667123 & $1.56 \mathrm{E}+06$ & 49.87447819 \\
\hline 21 & $1.20 \mathrm{E}+04$ & 172.7200473 & $2.03 \mathrm{E}+05$ & 98.68597108 & $1.71 \mathrm{E}+06$ & 49.89357969 \\
\hline 22 & $2.28 \mathrm{E}+04$ & 187.2732236 & $2.92 \mathrm{E}+05$ & 99.20376836 & $2.34 \mathrm{E}+06$ & 49.9485839 \\
\hline 23 & $5.84 \mathrm{E}+04$ & 197.8076514 & $4.38 \mathrm{E}+05$ & 99.59926443 & $2.73 \mathrm{E}+06$ & 49.96967324 \\
\hline 24 & $1.17 \mathrm{E}+05$ & 199.6806694 & $5.84 \mathrm{E}+05$ & 99.79741963 & $3.12 \mathrm{E}+06$ & 49.9847839 \\
\hline 25 & $1.75 \mathrm{E}+05$ & 194.2990466 & $7.30 \mathrm{E}+05$ & 99.9141813 & $3.90 \mathrm{E}+06$ & 49.99976631 \\
\hline 26 & $2.34 \mathrm{E}+05$ & 184.7559932 & $8.76 \mathrm{E}+05$ & 99.98113367 & $4.10 \mathrm{E}+06$ & 49.99977227 \\
\hline 27 & $2.92 \mathrm{E}+05$ & 173.7343822 & $1.02 \mathrm{E}+06$ & 99.99977159 & $4.68 \mathrm{E}+06$ & 49.9876968 \\
\hline 28 & $3.50 \mathrm{E}+05$ & 162.6918697 & $1.02 \mathrm{E}+06$ & 99.99964561 & $5.46 \mathrm{E}+06$ & 49.93426536 \\
\hline 29 & $4.09 \mathrm{E}+05$ & 152.2678274 & & & $5.46 \mathrm{E}+06$ & 49.93413039 \\
\hline 30 & $4.10 \mathrm{E}+05$ & 152.1306704 & & & & \\
\hline
\end{tabular}


stroke with a peak current of $100 \mathrm{kA}$ is illustrated in Figure 4.6. An increase in the WTG grounding impedance from $0.85 \Omega$ to $16.75 \Omega$ with increase in frequency from $5 \mathrm{kHz}$ to $1 \mathrm{MHz}$ is observed for WTG-1. The variation of the grounding impedance of WTG-2 is negligible for the frequency range of $5 \mathrm{kHz}$ to $580 \mathrm{kHz}$. However, a sudden surge in impedance is observed at $1 \mathrm{MHz}$ which is conferred due to the inductive reactance.

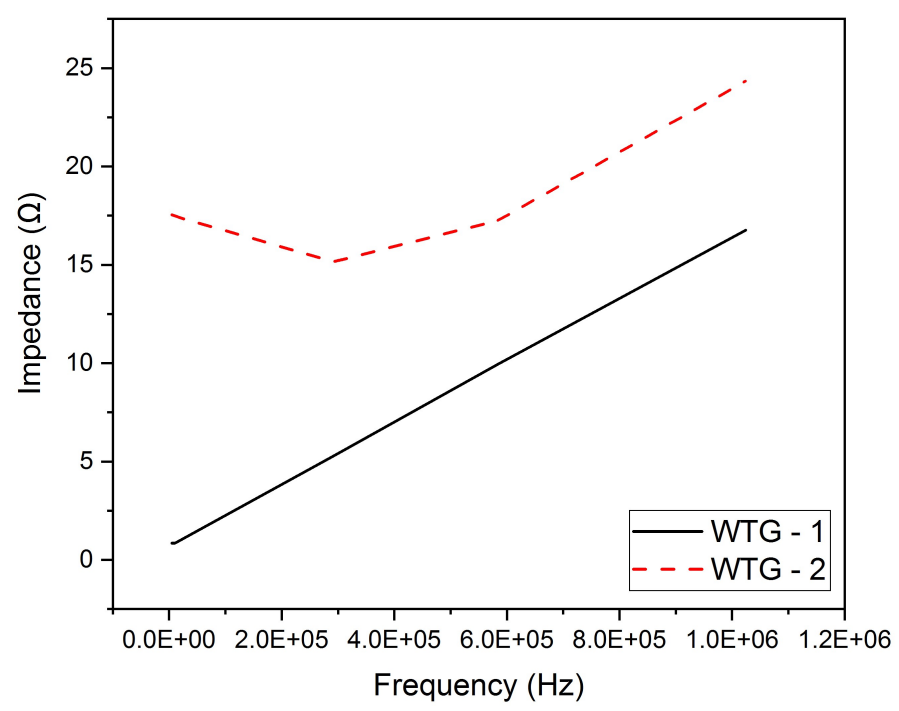

Figure 4.6: WTG earthing impedance for the first short negative stroke lightning current parameters.

The frequency response of the WTG earthing impedance for a subsequent short lightning discharge current is demonstrated in Figure 4.7. The frequency spectrum of the subsequent short stroke comprises of high frequency components of up to $5.46 \mathrm{MHz}$, compared to upper frequencies of $410 \mathrm{kHz}$ and $1 \mathrm{MHz}$ for the first short positive and negative lightning strokes. For WTG-1 the earthing impedance increases from $0.84 \Omega$ at $5.33 \mathrm{kHz}$ to $34.77 \Omega$ at $4.10 \mathrm{MHz}$ and then drops to $18.06 \Omega$ at $5.46 \mathrm{MHz}$. For WTG-2, the impedance is relatively constant, varying between a minimum of $17.5 \Omega$ at $5.33 \mathrm{kHz}$ and a maximum of $24.5 \Omega$ at $1 \mathrm{MHz}$, as illustrated in Figure 4.7 . 


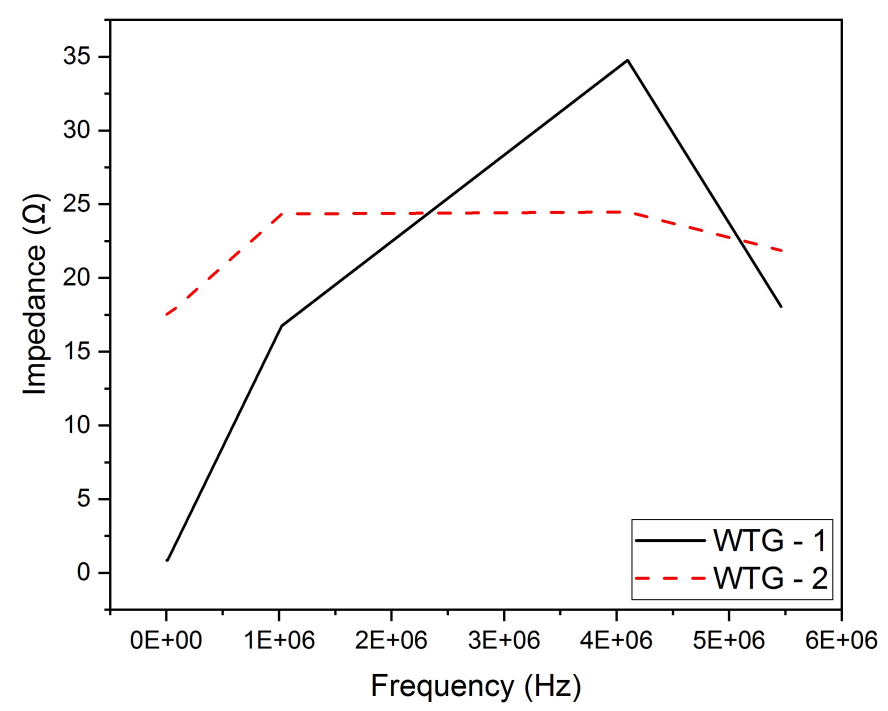

Figure 4.7: WTG earthing impedance for a subsequent short stroke lightning current parameters.

From the above analysis, it is observed that the WTG earthing impedance increases with an increase in the frequency for lower resistivity values. However, the impedance change is very minimal for the high soil resistivity sites. It is also observed that the high frequency components results in higher impedance than the high peak current values.

\subsubsection{Wind Turbine Earthing System Potential}

This section reveals the frequency response of the WTG grounding system potential rise for the considered lightning discharge current parameters and soil resistivity. Figures 4.8 to 4.14 illustrate the 3-D perspective of the earthing system profile potential above and in the nearby surrounding of the WTG earthing system. The impulse lightning current is injected at the WTG foundation centre which is approximately at the middle point of the profile.

The WTG grounding profile potential for WTG-1 for the first short positive current frequencies are depicted in Figures 4.8 and 4.9. As shown in 
Figure 4.8 , at the low frequency of $10.8 \mathrm{kHz}$, the potential magnitude at the WTG earthing grid is relatively constant representing an equipotential surface, with a peak value of approximately $130 \mathrm{kV}$ at $10.8 \mathrm{kHz}$. Interestingly, there are small spikes which represent the impulse injection point and the connection points of the stair case. Moreover, a sudden drop in the potential magnitude is detected in the surrounding area of the WTG earthing grid, a few meters away from the WTG foundation. A similar potential profile to the one in Figure 4.8 is found for a frequency of $5.2 \mathrm{kHz}$, with the exception of the spikes.

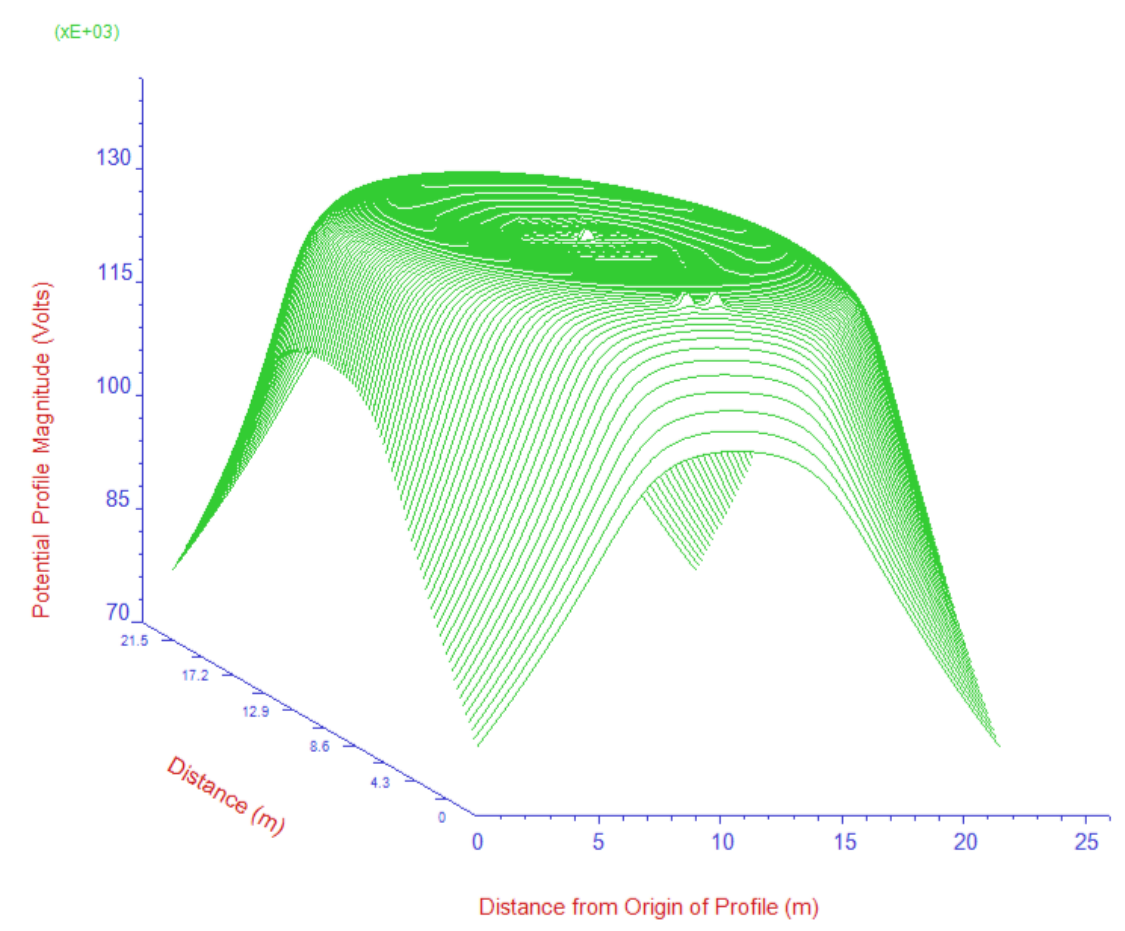

Figure 4.8: Scalar potential at $10.8 \mathrm{kHz}$ for the first short positive lightning stroke on WTG-1.

The behaviour of the earthing system is different at the high frequencies of a transient lightning discharge current. As depicted in Figure 4.9, there is a sharp rise at the centre of the profile and small spikes surrounding it at a frequency $410 \mathrm{kHz}$. Similar profiles are observed at frequencies 
of $117 \mathrm{kHz}$ and $350 \mathrm{kHz}$. The central spike is due to most of the current dissipating at the injection point. At high frequencies, the spikes in potential magnitude are due to a the inductance of the electrodes, which is no longer negligible. In addition, it can be observed that the maximum value of the potential spike increases with increase in frequency. The maximum value of the potential is about $290 \mathrm{kV}$ at $410 \mathrm{kHz}$.

For WTG-2, a WTG site with high soil resistivity, the earthing profile is almost equipotential for all frequencies, with small spikes on the WTG earthing system at high frequencies. The potential magnitudes are close to $3000 \mathrm{kV}$ due to the increased impedance offered by the increased resistivity of the soil.

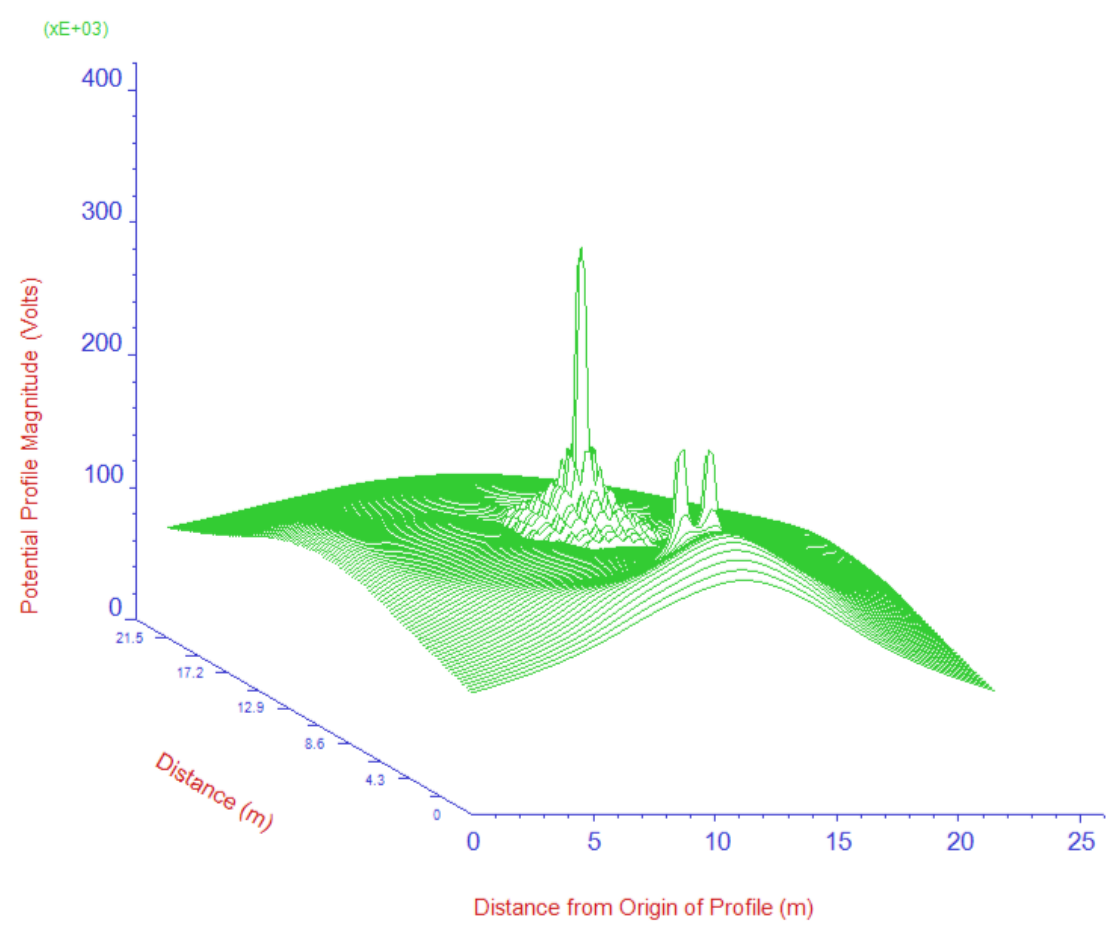

Figure 4.9: Scalar potential at $410 \mathrm{kHz}$ for the first short positive lightning stroke on WTG-1.

For the first short negative lightning discharge current with rise time 
less than that of the positive short stroke, the high frequency components are also increased. At low frequencies, the earthing profile is equipotential, with a maximum magnitude of about $40 \mathrm{kV}$, as shown in Figure 4.10. At $1 \mathrm{MHz}$, there are potential spikes similar to those of the positive current waveform, as depicted in Figure 4.11. However, it is observed that the peak value of the potential is higher than the first short positive current parameters.

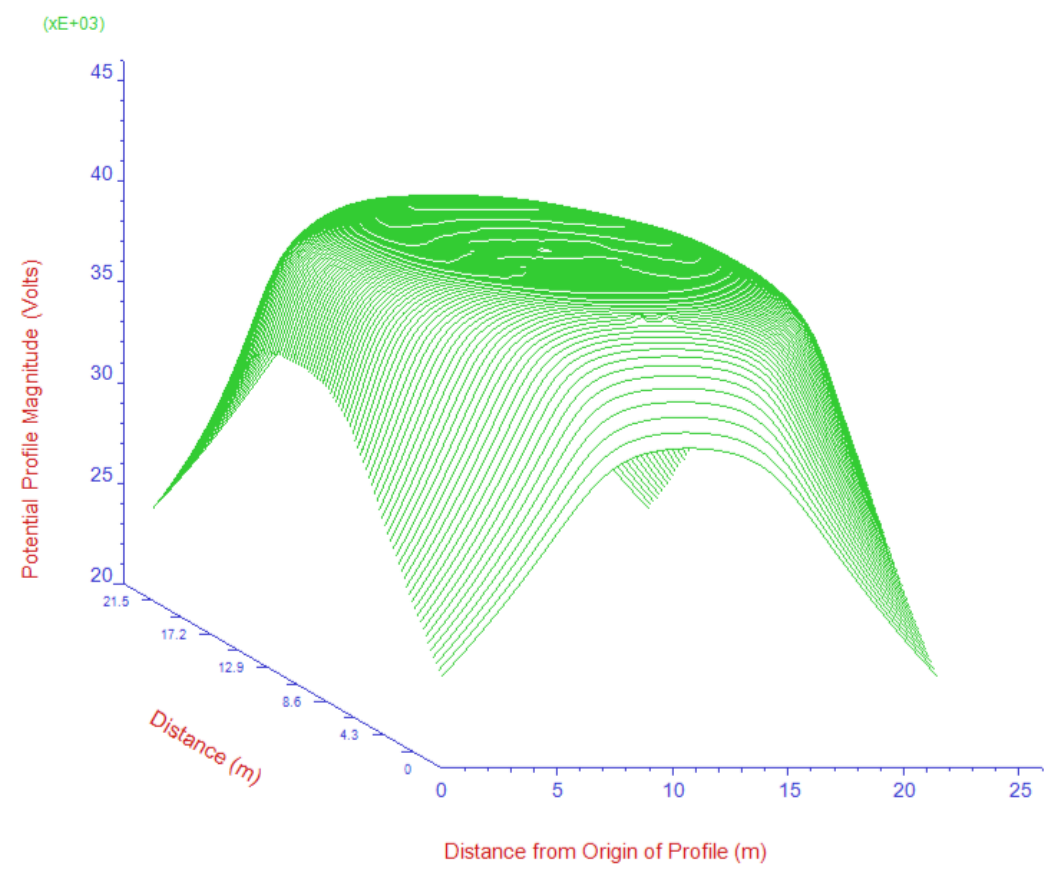

Figure 4.10: Scalar potential at $5 \mathrm{kHz}$ for the first short negative lightning stroke on WTG-1.

The subsequent short stroke has a much wider frequency spectrum compared to the other two wave shapes, as its rise time is fast at $0.25 \mu \mathrm{s}$. The high frequency component for the subsequent short stroke waveform is $5.46 \mathrm{MHz}$. At a lower frequency of $5.33 \mathrm{kHz}$, the voltage profile is equipotential with a peak of $10.5 \mathrm{kV}$, as depicted in Figure 4.12. At $1 \mathrm{MHz}$, potential spikes at the impulse current injection point and the stair 


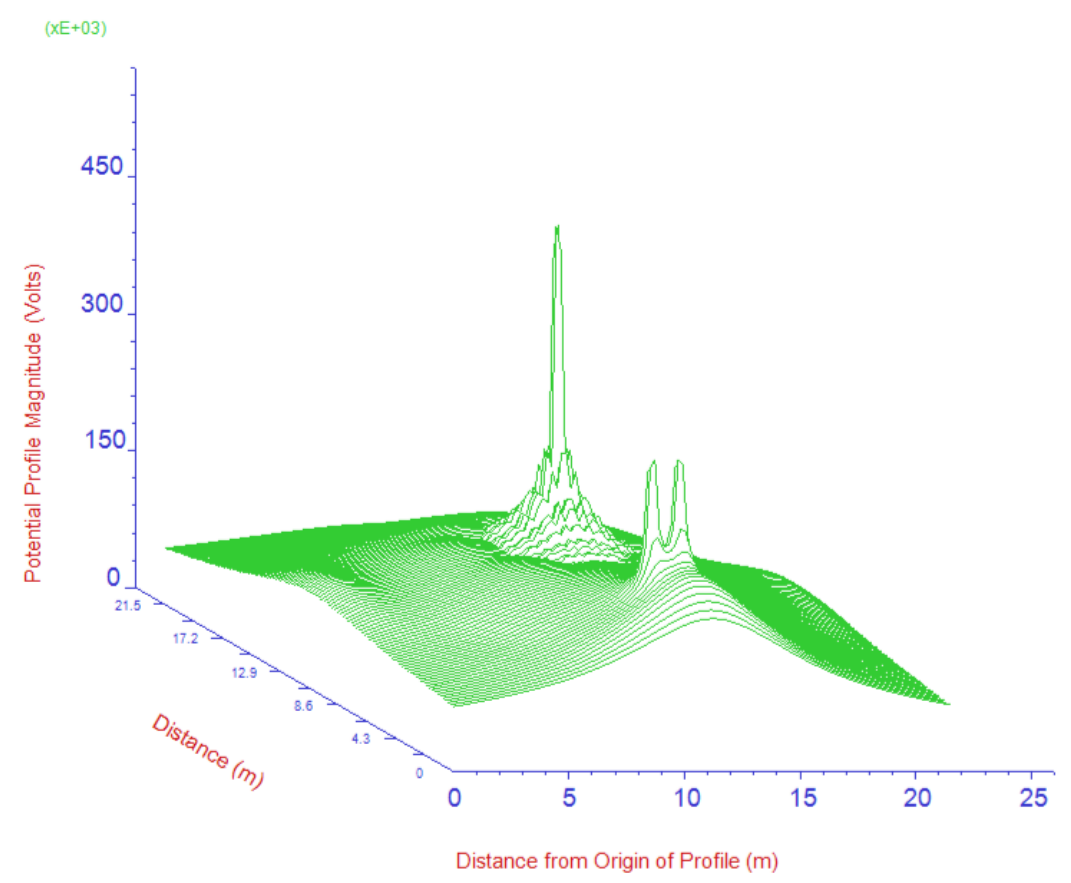

Figure 4.11: Scalar potential at $1 \mathrm{MHz}$ for the first short negative lightning stroke on WTG-1. 


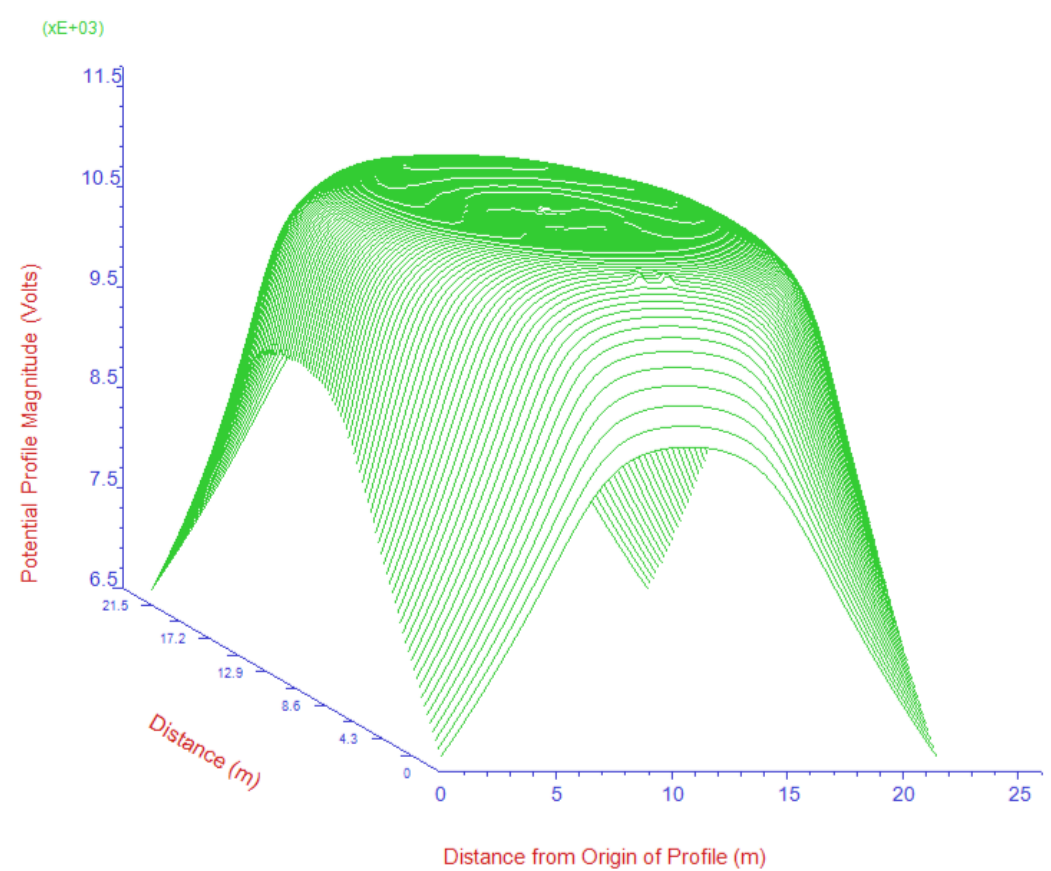

Figure 4.12: Scalar potential at $5.33 \mathrm{kHz}$ for a subsequent short lightning stroke on WTG-1. 
case connection points are observed, with the peak at the current injection point, as illustrated in Figure 4.13. The maximum potential magnitude at $1 \mathrm{MHz}$ is approximately $200 \mathrm{kV}$. However, at $5.46 \mathrm{MHz}$, the potential peak at the stair case connection point is greater than the current injection point with the peak value escalated to approximately to $450 \mathrm{kV}$, as depicted in Figure 4.14. The increase in potential is due to the increase in inductive component at higher frequencies at the staircase.

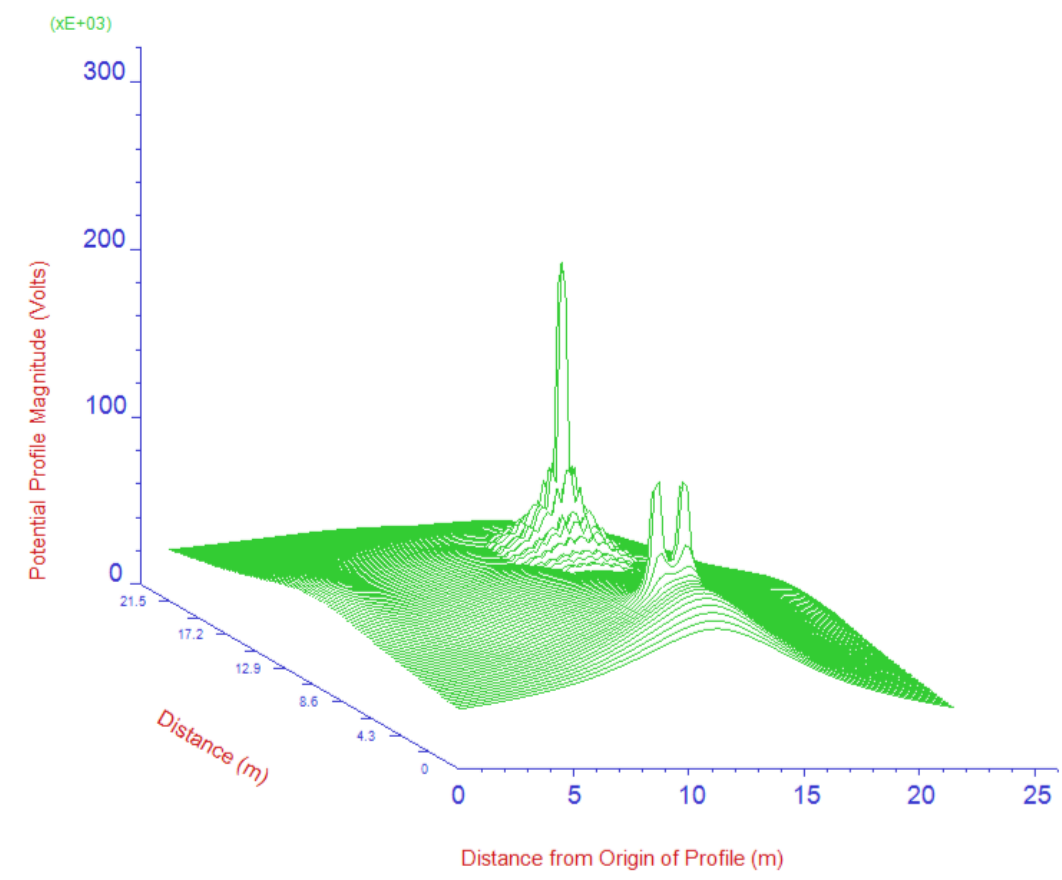

Figure 4.13: Scalar potential at $1 \mathrm{MHz}$ for a subsequent short lightning stroke on WTG-1.

The rise in potential around the wind turbine is equipotential at the low frequencies for all the lightning current wave shapes. This equipotential distribution is confined mainly due to negligible inductive component at the lower frequencies. However, at the higher frequencies, the potential profile is dominant at the impulse injection point and at high inductive reactance points. 


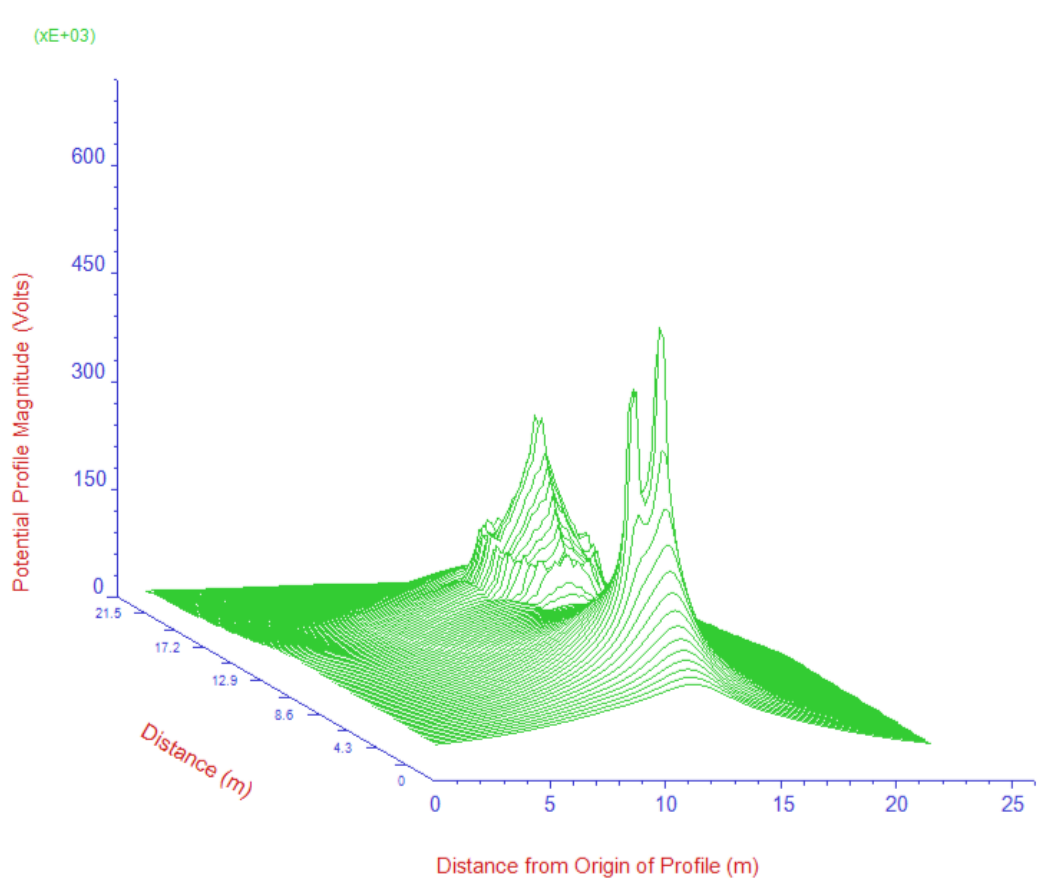

Figure 4.14: Scalar potential at $5.46 \mathrm{MHz}$ for a subsequent short lightning stroke on WTG-1.

The potential rise at the high soil resistivity wind turbine site is increased significantly in comparison to a low resistivity site. The potential distribution surrounding the WTG foundation is an equipotential for most of the frequencies, however, there are some localised potential spikes observed at the higher frequencies with relatively lower magnitudes.

The subsequent short lightning parameters should be considered for designing the WTG earthing system. Although the peak magnitude of the lightning discharge current is significantly low in comparison to the first short positive stroke and the first short negative stroke, the high frequency components are responsible for an increase in the potential rise and the earthing impedance. 


\subsection{Chapter summary}

This chapter illustrates an analysis of the wind turbine earthing system for different lightning discharge current wave shapes and soil resistivity values. The grounding impedance is minimum for the first short positive stroke current parameters for all the soil resistivity values compared to the first short negative and the subsequent short current wave shapes, although the peak current magnitude is highest for this wave shape. The high frequency components have major influence on the impedance due to dominant inductive reactance. The wind turbine grounding surface is equipotential at low frequencies and has potential spikes at higher frequencies. In addition, the type B earthing system provided an equipotential surface at higher soil resistivity values. It is evident from the results that there is a strong dependency of the potential profile on the impedance. 


\section{Chapter 5}

\section{Factors Determining the}

\section{Effectiveness of a Wind Turbine}

Generator Lightning Protection

\section{System}

Lightning is a natural phenomenon which can have direct and indirect risks for a wind turbine. The lightning strikes can cause transient overvoltages that can damage the WTG components and hurt people. It is imperative to consider the lightning strikes and their consequences in WTGs in the optimization phase of a wind farm. This chapter presents a framework to assess the effectiveness of wind turbine lightning protection systems at the wind farm design phase. Performing the analysis at this early stage reduces lightning-induced downtime, which leads to increased energy yield of the wind farm. Also, the assessment will improve the safety of the human operators and animals in the close proximity of a WTG. The research in this work shows that the grounding system plays a critical role in the wind turbine lightning protection system. For this reason an analysis of various influential parameters of a grounding system design is performed. 
Moreover, this chapter presents an analysis of various earthing electrode configurations for the lightning protection of a wind turbine grounding system. The IEC 61400-24 standard recommends a type-B earthing arrangement for the protection of wind turbines from lightning discharge currents. However, this research shows that the required impedance cannot be achieved with the recommended electrode lengths of the standard when typical soil resistivity values are taken into account. This chapter presents an analysis of different earth electrode configurations and lengths for lightning discharge current frequencies for different soil resistivity values based on frequency independent and dependent soil parameters.

\subsection{Introduction}

Lightning strikes can damage not just the individual wind turbine and its components, but also sections of a wind farm and parts of the grid [180]. This might lead to an increased downtime of WTGs. The increased downtime will not only increase the cost but also the indeterminacy of power generation from WTGs. To reduce lightning-related damages to WTGs, effective design and installation of LPSs [156] and assessment of LPS effectiveness for individual WTG are required.

The WTG LPS aims to dissipate lightning discharge currents safely in the grounding system, diverting it from the electrical and mechanical components in the WTG [137]. The installation of WTGs in a wind farm should consider the probability of lightning incidence and the soil resistivity of the WTG location. In the current practice of wind farm optimization, the lightning protection component is missing. Hence, the assessment of the site for lightning incidence will minimize damage.

The next step is to design an LPS following the international standards [17]. The design of LPS is based on the evaluation of risk assessment. The IEC 61400-24 standard defines four levels of lightning protection based on the risk assessment. Each lightning protection level (LPL) 
is associated with a set of design parameters. The parameters of LPL are based on the lightning discharge current parameters which have a critical role in LPS [181]. A lightning protection system consists of an external LPS, an internal LPS, and the grounding system [154].

The current practice is to design the external lightning LPS consisting of air termination and down conduction systems according to the LPL if an individual assessment of WTG site is possible [17]. Otherwise, a LPS is designed based in a more stringent LPL-1 parameters. The internal LPS is designed to avoid over-voltages and electromagnetic interferences due to lightning discharge currents. Finally, the grounding system ensures the proper functioning of all the protection systems.

A WTG grounding system ensures human safety and equipment protection in the event of a power system fault or lightning strike on a WTG [156]. To protect the wind turbine and interconnected electrical equipment, a grounding system that provides a low impedance path to ground is necessary [21].

The effectiveness of a WTG LPS is determined by the individual effectiveness of the LPS components. However, the effectiveness of the external and internal lightning protection systems are determined by the design and installation of the WTG LPS. The effectiveness of a grounding system is critical as it ensures the effectiveness of other components of an LPS. The aim of a grounding system according to the IEC 61400-24 standard [17] is to achieve a resistance of less than $10 \Omega$ at low frequencies. However, this doesn't consider the grounding system behaviour at higher frequencies, which represent the fast transients of the lightning discharge currents. Moreover, the evaluation of potential distribution is important to protect human operators and livestock from step and touch voltages. 


\subsection{Assessment framework for WTG lightning protection system}

This section introduces an assessment framework for WTG lightning protection system. The factors determining the effectiveness of a WTG LPS are depicted in Figure 5.1.

The effectiveness, $E_{L P S}$, of a WTG lightning protection system is a function of several individual components and can be defined as:

$$
E_{L P S}=f\left(E_{X}, E_{I}, E_{G}\right)
$$

where $E_{X}$ is the effectiveness of the external lightning protection system, $E_{I}$ is the effectiveness of the internal lightning protection system and $E_{G}$ is the effectiveness of the grounding system.

\subsubsection{Effectiveness of external lightning protection system}

The protection of a WTG from a direct lightning strike is achieved by the external lightning protection system, consisting of an air-termination system and a down conduction system. The effectiveness of the external lightning protection system is a function of effectiveness of the air termination system $E_{A}$ and the effectiveness of the down conduction system $E_{D}$ :

$$
E_{X}=f\left(E_{A}, E_{D}\right)
$$

$E_{A}$ is the effectiveness of the air termination system and $E_{D}$ is the effectiveness of the down conduction system.

\subsubsection{Effectiveness of air-termination system}

The purpose of an air-termination system is to protect the WTGs from direct lightning strikes [154]. As the lightning strikes are uncontrolled, an effective air-termination system is required to safeguard the WTG. The effectiveness of the air-termination system has two parts: the sizing effective- 


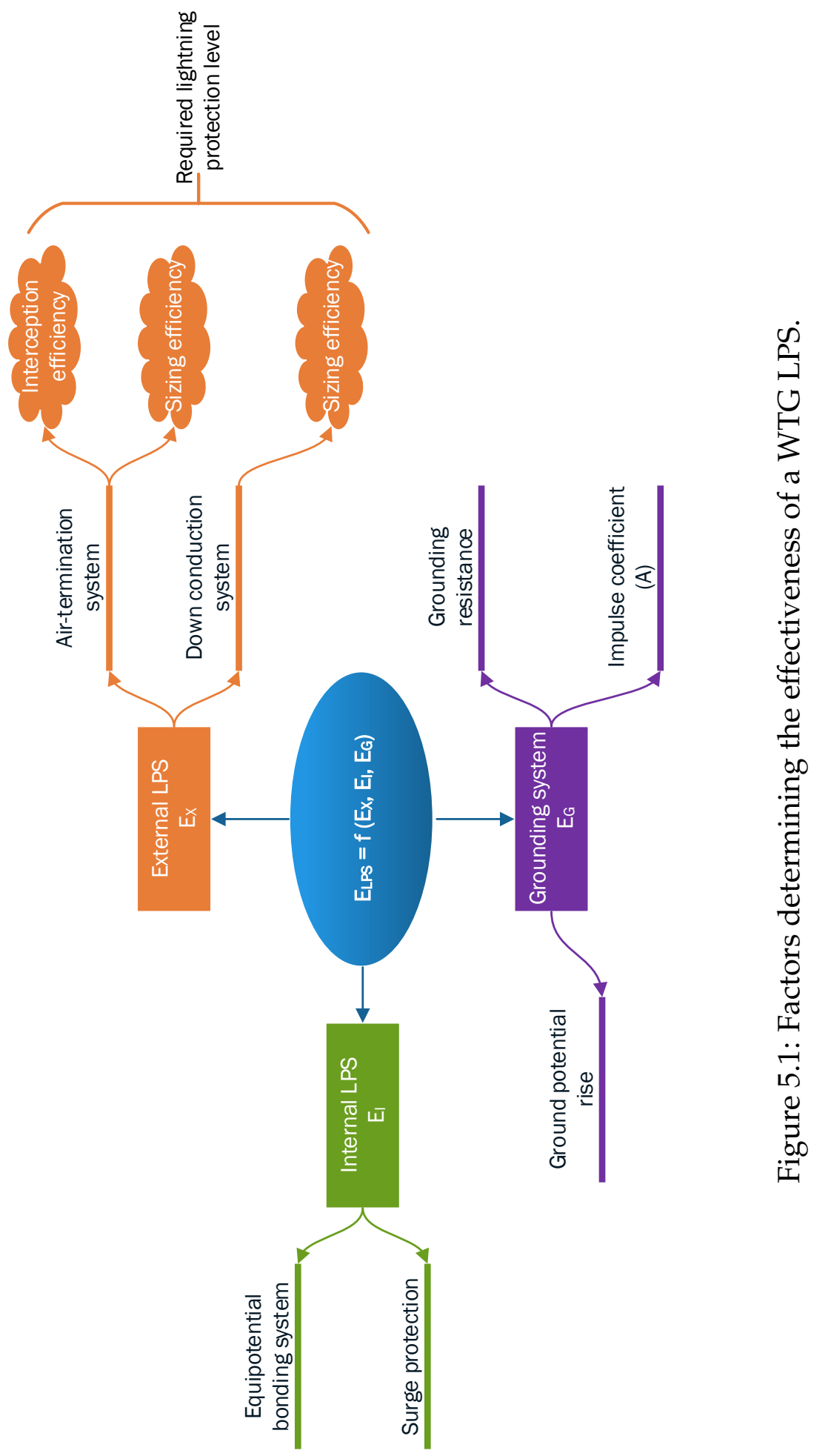


ness and interception effectiveness. The effectiveness of an air-termination system can be calculated by:

$$
E_{A}=E_{I C} \cdot E_{S A}
$$

where $E_{I C}$ is the interception effectiveness of the air termination system and $E_{S A}$ is the sizing effectiveness of the air-termination system.

\subsubsection{Effectiveness of down conduction system}

The down conduction system provides an electrically conductive path between the air-termination system and the grounding system. The purpose of a down conduction system is to discharge the lightning currents to the grounding system safely. Also, the down conduction should provide multiple parallel conductive paths to ground by offering low electrical resistance. The effectiveness of a down conduction system is dependent only on $E_{S D}$, the sizing effectiveness of the down conduction system:

$$
E_{D}=E_{S D}
$$

\subsubsection{Effectiveness of internal lightning protection system}

The internal lightning protection system of a WTG consists of equipotential bonding, spatial shielding and separation distance, cable routing and cable shielding and the installation of coordinated surge protection devices (SPDs). The effectiveness of an internal lightning protection system can be calculated as:

$$
E_{I}=f\left(E_{E B}, E_{S P D}\right)
$$

where $E_{E B}$ is the effectiveness of the equipotential bonding system and $E_{S P D}$ is the effectiveness of the surge protection. 


\subsubsection{Effectiveness of grounding system}

The function of the grounding system is to dissipate the lightning discharge currents into the ground without dangerous potential values [182]. An effective grounding system will ensure proper function of an LPS, protecting persons and animals [183]. The influencing factors determining the ground potential rise and impedance are soil resistivity [184], grounding electrodes and lightning discharge current parameters [185]. The effectiveness of a grounding system can be evaluated as:

$$
E_{G}=f(G R, A, G P R)
$$

where $G R$ is the resistance of the WTG grounding system at low frequency, $A$ is the impulse coefficient and $G P R$ is the ground potential rise.

The design principles described in IEC 61400-24 [17] are based on IEC 62305-3 [88], which was initially designed for general structures consisting of houses and buildings [169]. The foundations of buildings are typically larger than those of WTGs, allowing large diameter ring electrodes [168].

The main requirement of the grounding system according to IEC 61400-24 [17] is to achieve a resistance value of less than $10 \Omega$ at low frequencies before connecting the grounding system to the rest of the wind farm. This job is challenging at wind turbine sites with high soil resistivity.

The grounding systems designed for power frequencies have a task of achieving low resistance values at steady state or low-frequency analysis [186]. The low-frequency performance assures the effectiveness of the grounding system at the wave trail of the lightning discharge current waveform [181]. However, high-frequency components are present during the fast rise times of the lightning discharge current. The effectiveness of the grounding system for transients can be evaluated by the impulse coefficient (A), given by [187]:

$$
A=\frac{Z}{R}
$$


where $Z$ is the impedance of the grounding system at high frequencies and $R$ is the resistance of the grounding system at low frequencies.

The other significant parameter to assess the effectiveness of the grounding system is to evaluate the ground potential rise with reference to a remote earth [188]. This parameter also represents the grounding system behaviour for transients, which pose a hazard to nearby humans and animals. The potential distributions help in evaluating the step and touch voltages. Step-voltage is defined as the potential difference between two points separated by a distance [189]. The touch voltage is the potential difference between GPR of a system system and the potential of a person touching a structure with his hand which might be at a different potential at the same time [190]. The step $\left(U_{S}\right)$ and touch $\left(U_{t}\right)$ voltages are illustrated in Figure 5.2.

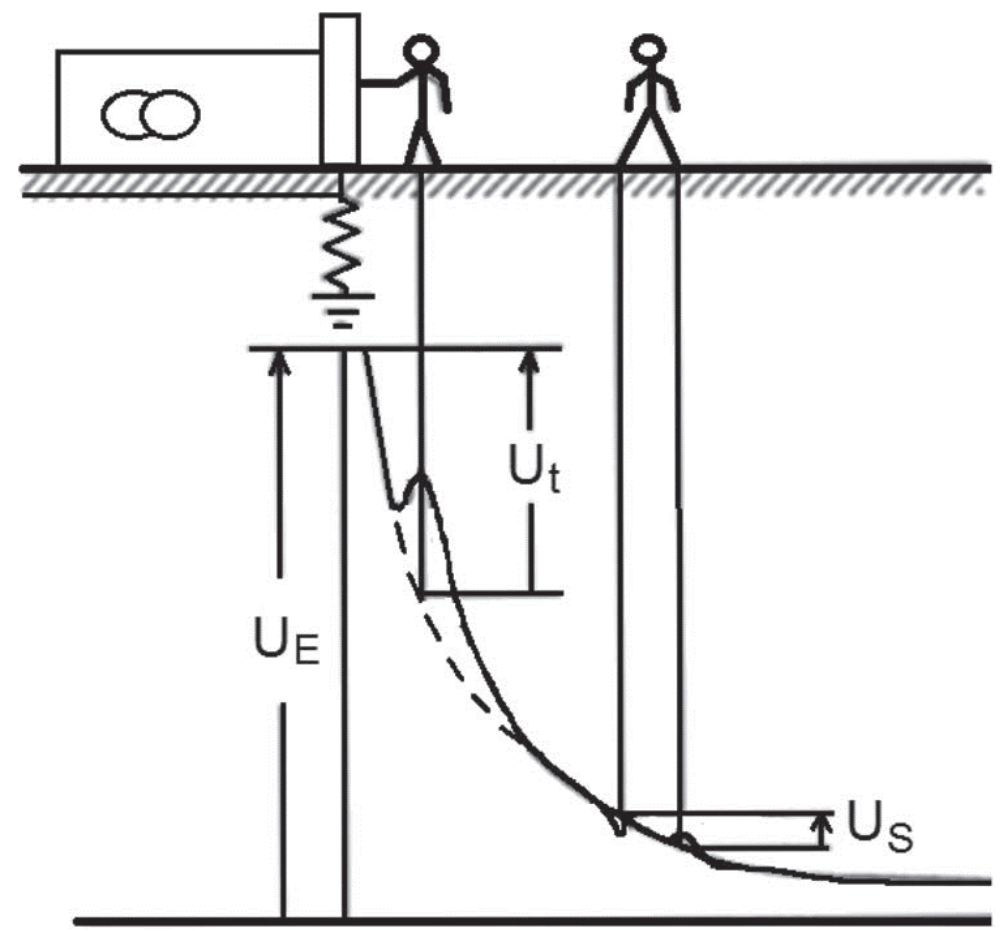

Figure 5.2: Step and touch voltages [190]. 
The limiting values for the step and touch voltages are defined for defined for the power frequencies [189]. However, there is no guidance on the allowable limits for the step and voltages at high frequencies generated by the lightning discharge currents. Hence, this study considered potential distribution to analyse these quantities. The human-worst step voltage [152] defined as the maximum potential difference between the observation point and within a $1 \mathrm{~m}$ stride length of that point is evaluated.

\subsection{Case Study and Simulation Results}

This case study presents an evaluation of the effectiveness of individual components of an LPS. The effectiveness calculations of air-termination and down conduction system are based on the lightning protection level of IEC 61400-24. The effectiveness of the internal LPS is dependent on the individual WTG installations. However, the effectiveness of the grounding system varies significantly depending on the LPL, soil resistivity value, modelling of soil structure, type of grounding electrodes, length of the grounding electrodes, modelling of the grounding system, and frequency, temperature and moisture dependency of soil resistivity.

For an air-termination system designed according to the IEC 6140024 [17] and IEC 62305-3 [88] standards, the effectiveness for different lightning protection levels is calculated as depicted in Table 5.1 [191].

Table 5.1: Effectiveness of air-termination system

\begin{tabular}{ccccc}
\hline Lightning protection level (LPL) & I & II & III & IV \\
\hline Sizing effectiveness & 0.99 & 0.98 & 0.95 & 0.95 \\
Interception effectiveness & 0.99 & 0.97 & 0.91 & 0.84 \\
Total effectiveness & 0.98 & 0.95 & 0.86 & 0.8 \\
\hline
\end{tabular}

In consonance with the IEC 61400-24 standard, the design of the LPS 
for the WTGs commences with the risk assessment process. For a given LPL, if the tolerable risk is below the limit, the protection system for that particular LPL is designed. However, the current practice in the industry is not to perform the risk assessment for individual WTG sites and hence to design a level-1 LPS [192], which results in the effectiveness of 0.98 , as provided in Table 5.1 .

Similarly for the down conduction system designed according to IEC 61400-24 [17] and IEC 62305-3 [88] standards, the effectiveness for different lightning protection levels is shown in Table 5.2.

Table 5.2: Effectiveness of down conduction system

\begin{tabular}{ccccc}
\hline Lightning protection level (LPL) & I & II & III & IV \\
\hline Sizing effectiveness & 0.99 & 0.98 & 0.95 & 0.95 \\
\hline
\end{tabular}

The effectiveness of the internal LPS mainly depends on the equipotential bonding system and the coordinated surge protection devices (SPDs). The effectiveness of the equipotential bonding system is evaluated by measuring the resistance between all the conductive parts and the equipotential bus bar. The recommended resistance value is less than $1 \Omega$ [90]. However, resistance of the order of $\mathrm{m} \Omega$ is preferable. Whether it is a lightning transient or power system fault current, the over-voltages appear at the terminals of the electrical, electronic and control systems installed in the WTGs. The proper installation of coordinated SPDs according to the lightning protection zones [17] and voltage levels will protect the end equipment.

The goal of assessing an LPS is to reduce the downtime and hence increase the energy yield of a WTG. Wind farm designers, in current practice, do not consider this factor while optimizing the wind turbines in a wind farm [193]. However, factors that can be controllable by wind farm designers are the installation of WTGs at low lightning incidence locations and the design of a sound grounding system. An important element of 
the WTG LPS that ensures the effectiveness of all other components is the grounding system. By achieving a better grounding system consisting of a low soil resistivity site, better soil structure modeling and better electrode arrangement and dimensions improves the overall effectiveness of the LPS. Hence, the effectiveness of a grounding system is analysed in detail for several scenarios.

The first short negative lightning stroke is considered for the lightning discharge current wave shape with $1 \mu$ s rise time and $200 \mu$ s time to half value. The FFTSES module of CDEGS is used to identify the frequency spectrum of the lightning discharge current wave. Amongst the computed frequencies, $5 \mathrm{kHz}, 1 \mathrm{MHz}$, and $4.52 \mathrm{MHz}$ are selected for this analysis, as $1 \mathrm{MHz}$ represents the dominant frequency when the time-domain waveform is near its peak value and $4.52 \mathrm{MHz}$ represents the dominant frequency of the time-domain waveform closest to the upper limit of the frequency range in which the frequency dependent soil parameters are valid. $5 \mathrm{kHz}$ represents the dominant frequency when the time-domain waveform is near $50 \%$ of its peak value during the fall time, as illustrated in Fig 5.3. The simulations for this analysis were performed in the frequency domain using FFTSES and HIFREQ modules of the CDEGS software package [152].

The perspective view of the WTG grounding system model used for the simulations is shown in Figure 2.9 in Section 2.5.3. The grounding system evaluation parameters for this analysis are low-frequency resistance, impulse coefficient, peak potential magnitude, and the step voltages. The permissible limits for step voltages cannot be compared against the standard as there is no fault clearance time [194] associated with lightning discharge currents. The grounding system designs for different soil resistivity values, soil stratification models, electrode configuration and frequency dependent soil parameters are analysed in this work. 


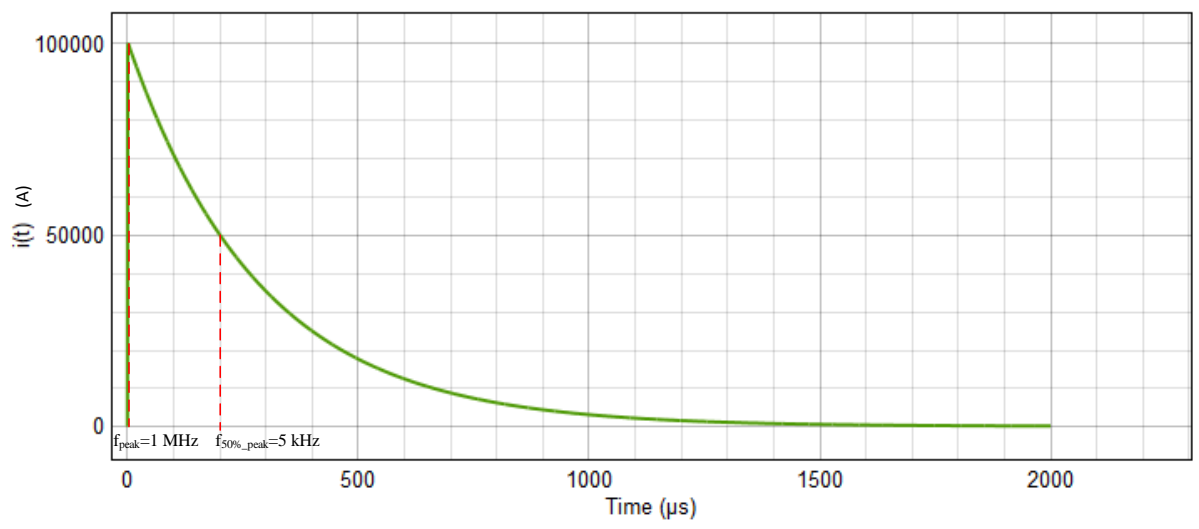

Figure 5.3: Representation of frequencies of lightning discharge current.

\subsubsection{Earth electrode configurations}

Figures 5.4 to 5.6 depict various earth electrodes for WTG grounding, which can be used individually or in combination to design a compliant earthing system. The back bone of a type-B earthing system, recommended by the IEC 61400-24 standard is the ring electrode, which is designed around the foundation of the WTG and connected to the tower base through the rebar. The mean radius of the ring electrode is approximately the radius of the wind turbine tower base. These electrodes are buried in the ground about one to two meters deep.

Horizontal and vertical electrodes are also commonly used; IEC 6140024 recommends the number of horizontal and vertical electrodes should be equal to the number of down conductors, with a minimum of two electrodes. The four cross and eight cross electrode configurations, shown in Figures $5.4 \& 5.5$, are connected through vertical electrodes and are also used for earthing system designs. Finally, an earthing grid with horizontal and vertical electrodes buried below ground and connected through vertical electrodes is shown in Figure 5.6. 


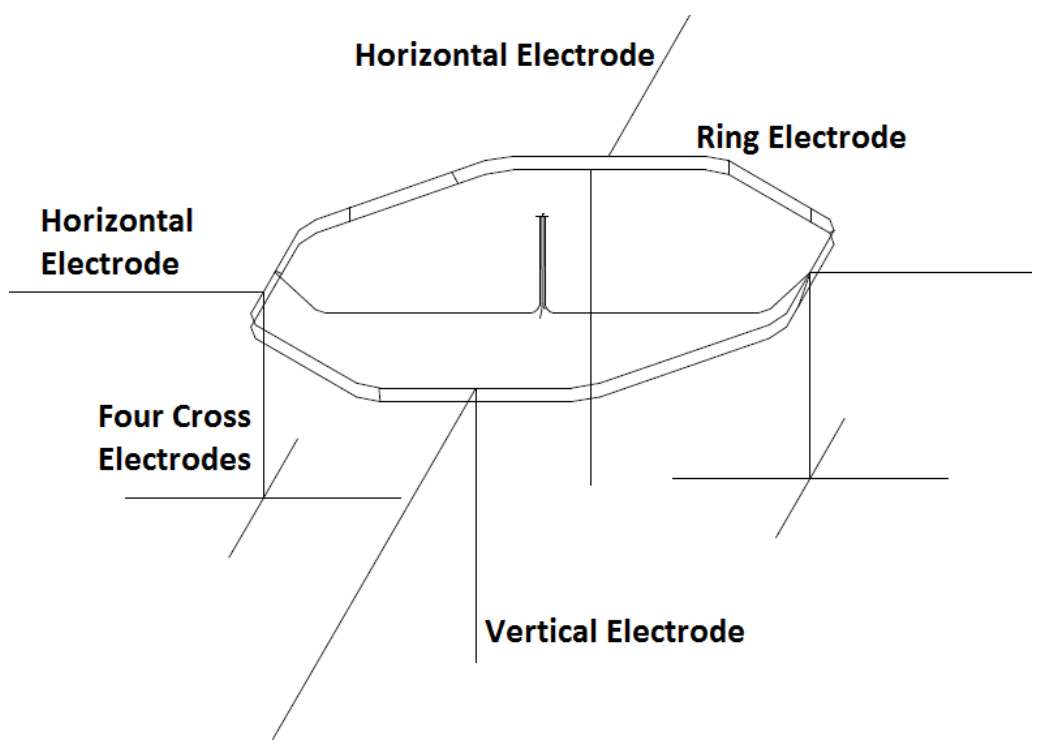

Figure 5.4: Perspective view of WTG commonly used earthing electrode structures (not to scale).

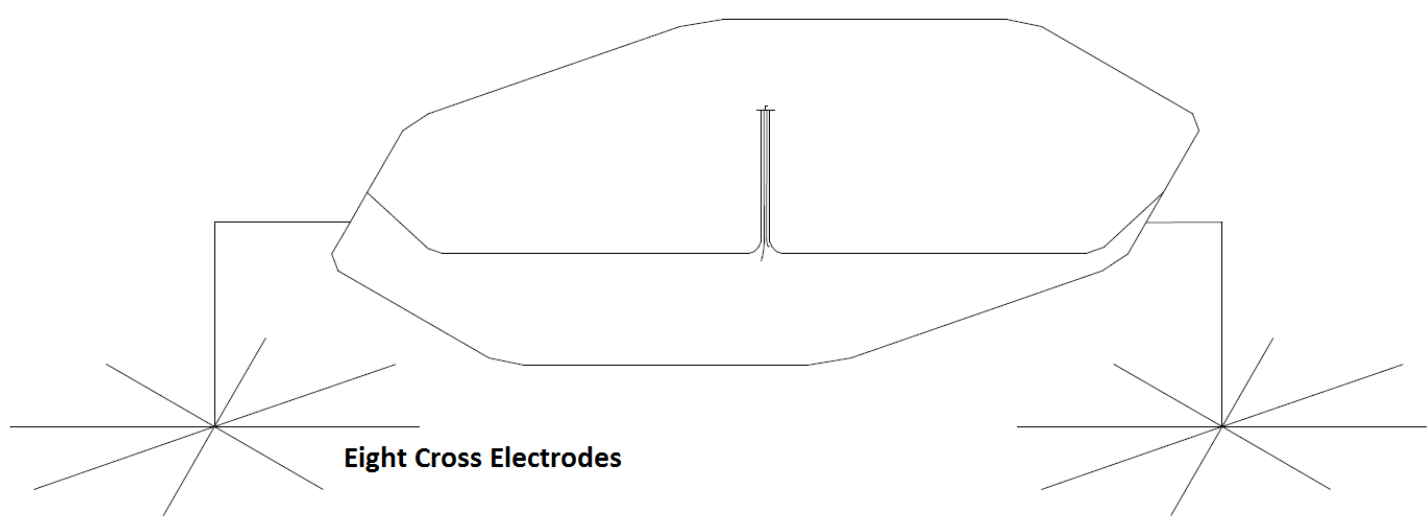

Figure 5.5: Perspective view of WTG earthing system with eight cross electrodes (not to scale). 


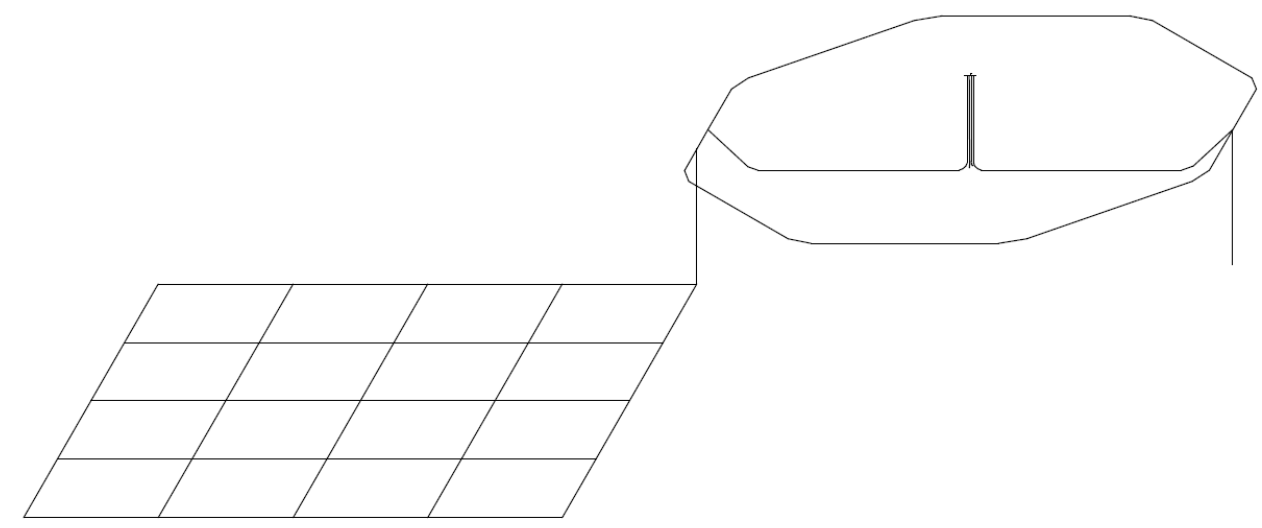

Figure 5.6: Perspective view of WTG earthing system with grid electrodes (not to scale).

\subsubsection{Frequency dependent soil parameters}

Table 5.3 demonstrates the frequency dependence of soil resistivity values calculated by using the method described in Section 3.3. The effective value of the soil resistivity reduces with increase in frequency. As an example, soil with resistivity of $10 \mathrm{k} \Omega \mathrm{m}$ at $100 \mathrm{~Hz}$ has resistivity of only $1120 \Omega \mathrm{m}$ at $1 \mathrm{MHz}$.

Table 5.4 depicts the frequency dependent relative permittivity values for the soil. At low frequencies, the value of relative permittivity can be as high as 192.2, which increases the capacitive behaviour of the earthing system.

\subsubsection{Effectiveness of the WTG earthing system for various LPLs}

In the first scenario, the grounding system designs for various lightning protection levels according to IEC 61400-24 are compared. The length of the electrodes is determined from Figure 2.10 in Section 2.5.3 according to the low-frequency soil resistivity value. As the mean radius of the ring electrode is bounded by the wind turbine tower foundation, additional 
Table 5.3: Frequency Dependent Soil Resistivity

\begin{tabular}{|c|c|c|}
\hline $\begin{array}{c}\text { Soil Resistivity } \\
\text { at } 100 \mathrm{~Hz} \\
{[\Omega . \mathrm{m}]}\end{array}$ & Frequency $[\mathrm{Hz}]$ & $\begin{array}{c}\text { Frequency Dependent } \\
\text { Soil Resistivity } \\
{[\Omega \mathrm{m}]}\end{array}$ \\
\hline 100 & 5000 & 99.14 \\
\hline 100 & 1000000 & 78.44 \\
\hline 100 & 4000000 & 59.63 \\
\hline 1000 & 5000 & 955.53 \\
\hline 1000 & 1000000 & 403.84 \\
\hline 1000 & 4000000 & 215.75 \\
\hline 2000 & 5000 & 1856.68 \\
\hline 2000 & 1000000 & 579.96 \\
\hline 2000 & 4000000 & 284.52 \\
\hline 10000 & 5000 & 8000.48 \\
\hline 10000 & 1000000 & 1120.10 \\
\hline 10000 & 4000000 & 487.29 \\
\hline
\end{tabular}

Table 5.4: Frequency Dependent Relative Permittivity

\begin{tabular}{|c|c|c|c|}
\hline Frequency (Hz) & 5000 & 1000000 & 4000000 \\
\hline Relative Permittivity & 192.20 & 31.56 & 18.68 \\
\hline
\end{tabular}


electrodes are needed for high soil resistivity sites. However, the minimum lengths can not guarantee low impedance for the WTG grounding system. Hence, this work considers different electrode configurations with varied lengths to calculate the impedance. Moreover, this work evaluates the high-frequency impedance in the second scenario for all the electrode configurations.

The low-frequency resistance of the WTG grounding system increases with increase in soil resistivity as shown in Figure 5.7. It can be observed that the low-frequency resistance is constant up to $500 \Omega \mathrm{m}$ soil resistivity for all LPLs. This is due to the same length of earth electrode requirement up to $500 \Omega \mathrm{m}$ soil resistivity. However, the resistance increases from $1000 \Omega \mathrm{m}$ due to the change in electrode lengths for various LPL. For

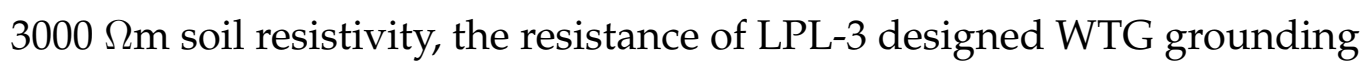
system is twice that of LPL-1.
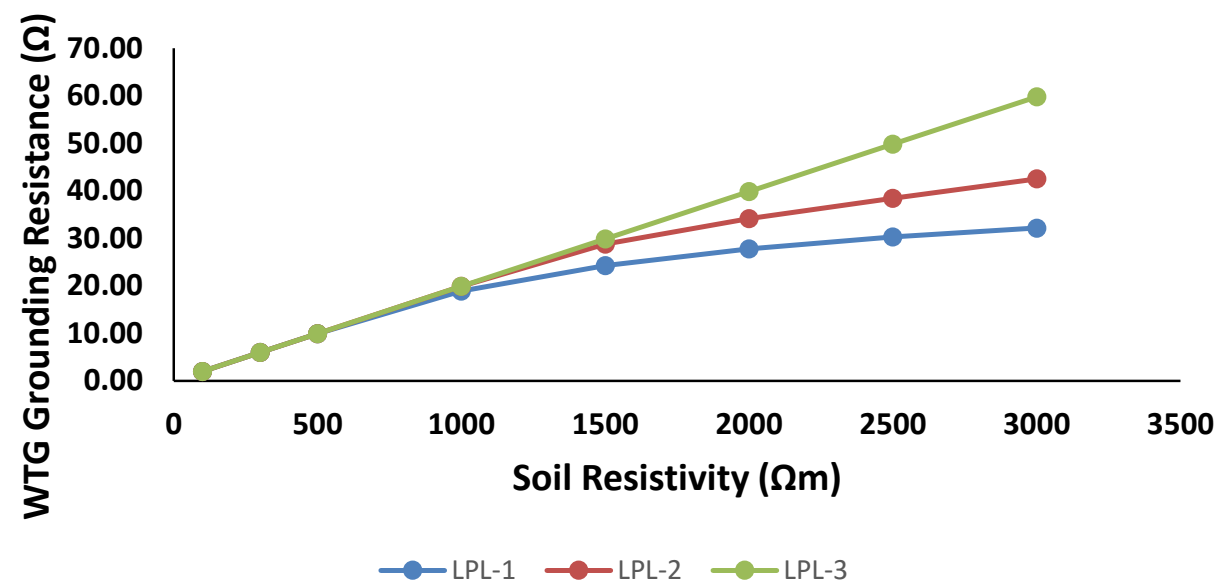

Figure 5.7: WTG grounding resistance for different lightning protection levels.

The impulse coefficient is as high as 10 for low soil resistivity values and drops to less than 2 for soil resistivities beyond $1000 \Omega \mathrm{m}$, as depicted in Figure 5.8. The high impulse coefficients at low soil resistivity are due 
to the high impedance values at higher frequencies and relatively small resistance values at low frequencies. An impulse coefficient value of 1 represents resistive behaviour, less than 1 represents capacitive behaviour, and greater than 1 represents inductive behaviour of the grounding system. The higher impulse coefficient at low soil resistivity values indicates the inductive response of the grounding system at high frequencies. However, the low values of impulse coefficient at higher soil resistivity values indicate poor behaviour of the grounding system at low frequencies.

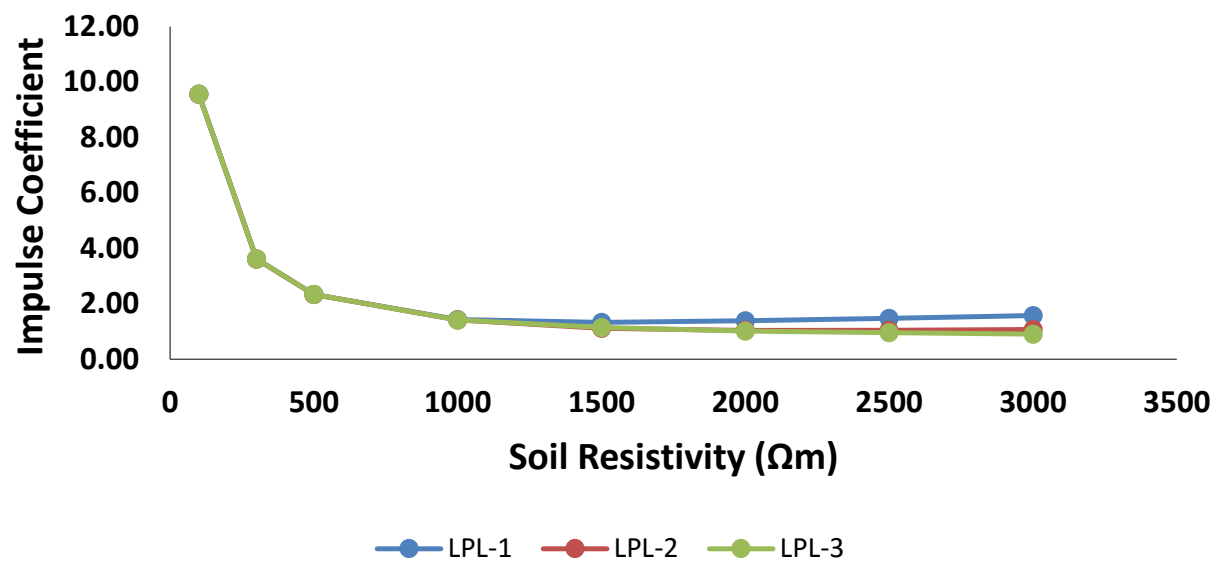

Figure 5.8: Impulse coefficient of the WTG grounding system for first short negative lightning discharge current parameters.

The potential distribution around the WTG foundation is observed to be equipotential for all the LPLs at low frequencies, as depicted in Figure 5.9. However, at higher frequencies, the potential profile experiences a significant change within a small area with high potential spikes, as illustrated in Figure 5.10. The peaks are due to greater current dissipation at the impulse injection points and the connection point of the staircase to the WTG grounding grid.

It is critical to evaluate the peak potential magnitude and the step voltages at the WTG grounding system and the area surrounding the WTG 


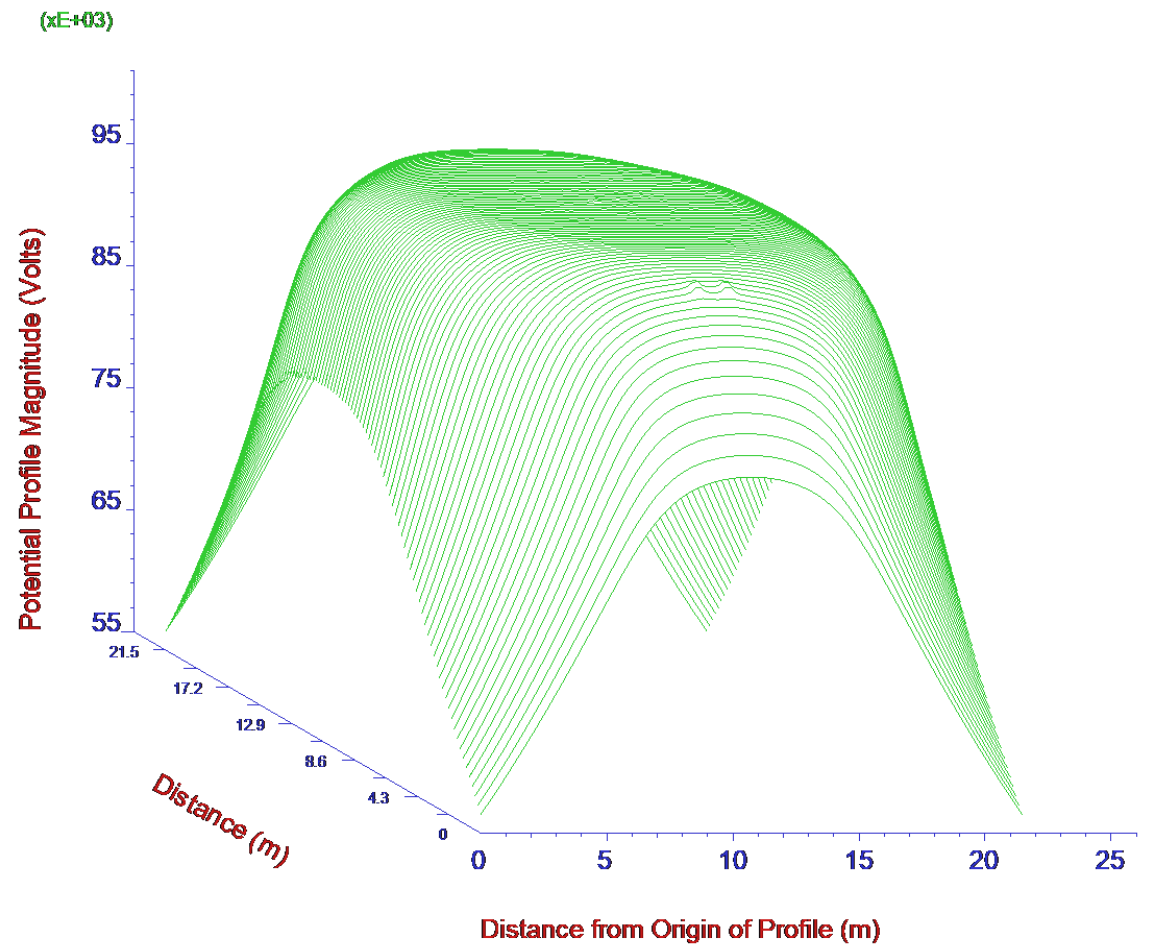

Figure 5.9: Potential distribution of the WTG grounding system at $5 \mathrm{kHz}$ for soil resistivity of $100 \Omega \mathrm{m}, \mathrm{LPL}-1$. 


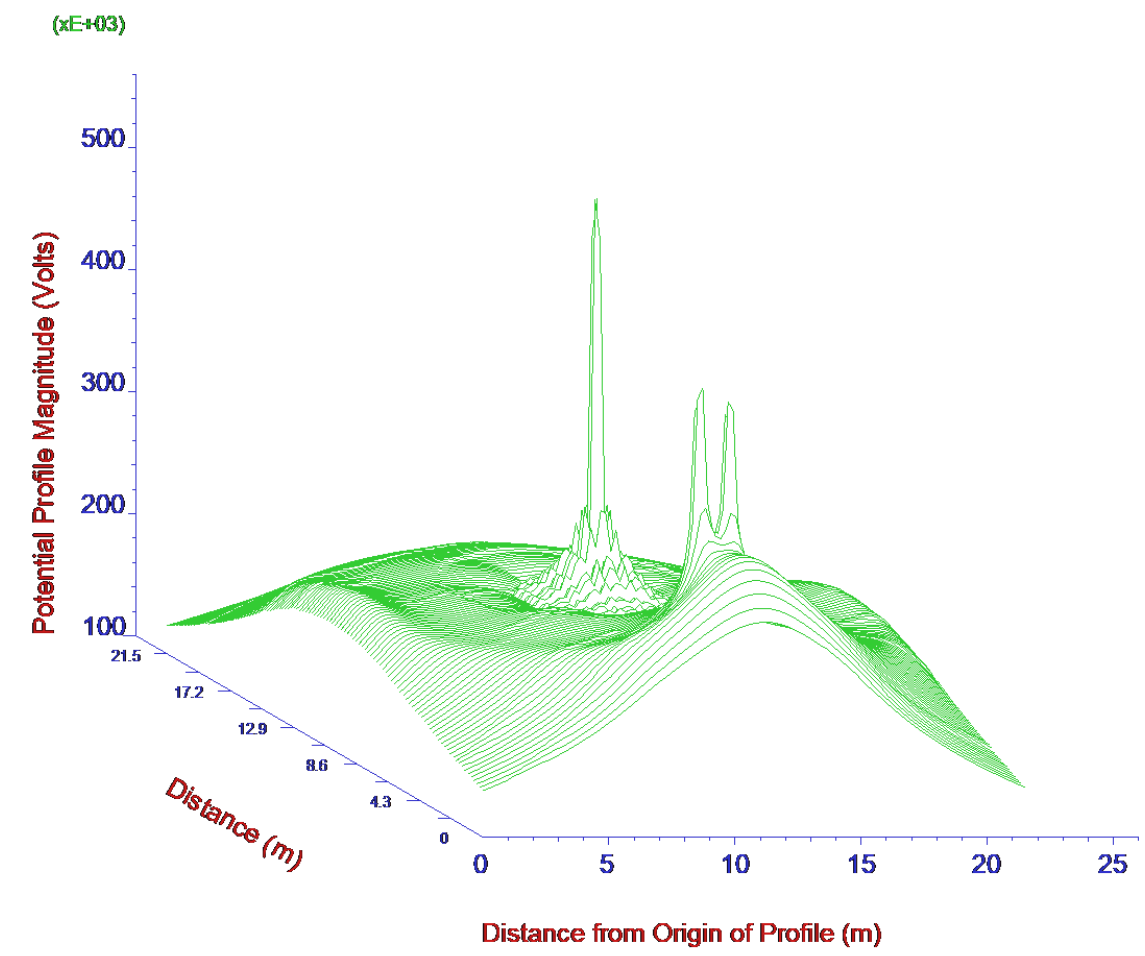

Figure 5.10: Potential distribution of the WTG grounding system at $1 \mathrm{MHz}$ for soil resistivity of $100 \Omega \mathrm{m}, \mathrm{LPL}-1$. 
foundation. As depicted in Figure 5.11, the peak potential magnitude increases with increase in soil resistivity for all LPLs. Interestingly, the peak potential for the grounding system designed for LPL-1 is higher than LPL2 and LPL-3. Although a unit current injected into the WTG grounding system results in lower potential magnitude for LPL-2 and LPL-3 compared to LPL-1, it is the high peak current magnitude of LPL-1 that results in higher potential magnitudes. However, the potential values at higher frequencies are much more significant than low-frequency magnitudes not only due to high lightning discharge current magnitudes but also due to high-frequency inductive components. The predominant factor responsible for high potential profiles is high-frequency components of the lightning discharge current [35].

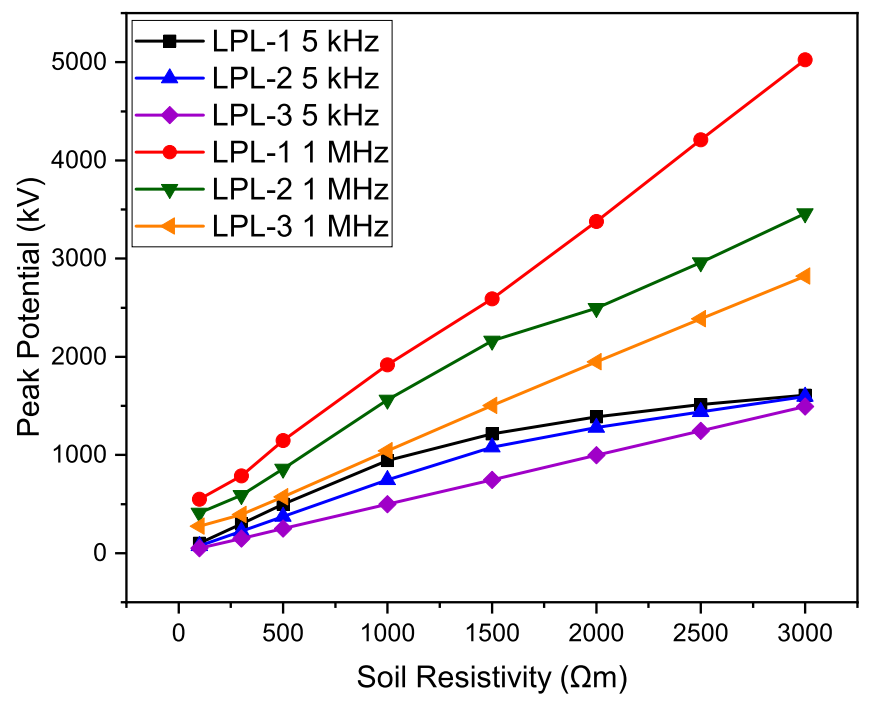

Figure 5.11: Peak potential magnitude at various lightning protection levels.

Now, the maximum possible step voltages are evaluated for all the LPLs, as depicted in Figure 5.12. The low step voltages at low-frequency are due to the equal distribution of electric field and current density throughout the WTG grounding grid. It is important to observe that at 
lower frequencies lower step voltages are noted for high soil resistivity values due to longer electrodes making an equal potential distribution. However, at high frequencies, the current density and electric field are mostly concentrated at the current injection point. Moreover, the current density is higher for WTG earth electrodes compared to the rebar. Hence, larger step voltages are observed at high frequencies.

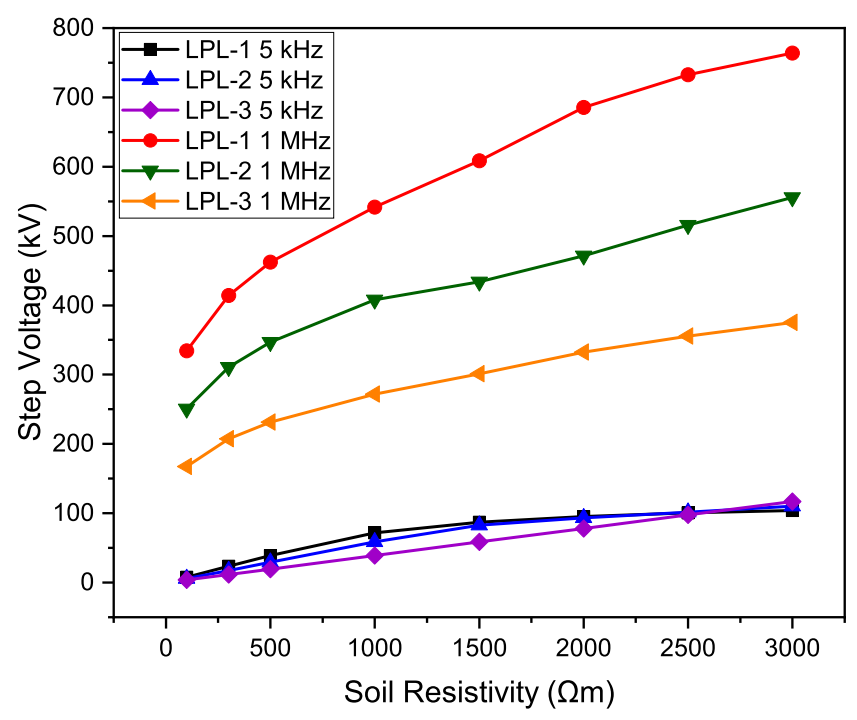

Figure 5.12: Step voltage for various lightning protection levels.

It is interesting to note the behaviour of a WTG grounding system designed to LPL-2 or LPL-3 which is subjected to a lightning discharge current that would normally only be experienced by a LPL-1 system. The low-frequency resistance of the LPL-2 WTG grounding system is $42.5 \Omega$ compared to $32.2 \Omega$ for the LPL-1 grounding system at a soil resistivity of $3000 \Omega \mathrm{m}$. Moreover, the low-frequency resistance further increases to $59.8 \Omega$ for LPL-3. The high-frequency impedance has no change for all the LPLs, due to the negligible effect of electrode length at higher frequencies. However, the impulse coefficient is higher for LPL-1 compared to LPL-2 and 3. This impaired performance is due to the compromise of low- 
frequency effectiveness of the grounding system. Further, the grounding system designed for LPL-2 and struck by LPL-1 parameters experiences a peak potential of $2125.55 \mathrm{kV}$ against $1594 \mathrm{kV}$ for LPL-2 parameters at $5 \mathrm{kHz}$. At $1 \mathrm{MHz}$, the potential magnitudes are $4611.77 \mathrm{kV}$ and $3458.81 \mathrm{kV}$. The increase in the potential for LPL-3 design experiencing LPL-1 stroke is higher than that of LPL-2. At $5 \mathrm{kHz}$, the potentials are $2988.63 \mathrm{kV}$ and $1494.31 \mathrm{kV}$, and at $1 \mathrm{MHz}$, the potential magnitudes are $640.29 \mathrm{kV}$ and $2820.13 \mathrm{kV}$.

\subsubsection{Effectiveness of the WTG earthing system for various electrode configurations}

In the second scenario, the performance of grounding systems for various electrode configurations is considered. The electrode configurations are: ring, horizontal, vertical, ring-horizontal, ring-vertical and grid type.

All the electrode configurations discussed above are analysed for soil resistivity values of $100 \Omega \mathrm{m}, 1000 \Omega \mathrm{m}, 2000 \Omega \mathrm{m}$, and $10 \mathrm{k} \Omega \mathrm{m}$. The analysis is carried out for both frequency independent and frequency dependent soil parameters. For frequency independent soil parameters, the soil resistivity value at $100 \mathrm{~Hz}$ and a relative permittivity of 1 are used for all the frequencies. However, the soil parameters are frequency dependent and have an influential effect on the WTG impedance. Hence the effect of frequency dependency on WTG impedance and phase angle are also studied.

Figures 5.13 to 5.20 illustrate the evaluated WTG grounding impedance and the phase angle for soil resistivity values of $100 \Omega \mathrm{m}, 1000 \Omega \mathrm{m}$, $2000 \Omega \mathrm{m}$, and $10 \mathrm{k} \Omega \mathrm{m}$ at $5 \mathrm{kHz}, 1 \mathrm{MHz}$, and $4.52 \mathrm{MHz}$. The computation of the impedance and phase angles for fixed and frequency dependent soil parameters are compared.

The WTG earth impedance and phase angle for $100 \Omega \mathrm{m}$ uniform soil resistivity are depicted in Figure 5.13. For $100 \Omega \mathrm{m}$ soil resistivity value, the recommended minimum length of the electrode according IEC 61400- 
24 [17] is $5 \mathrm{~m}$, which is fulfilled by the ring earth electrode alone. At $5 \mathrm{kHz}$, the low-frequency resistance is $2 \Omega$ for fixed soil resistivity. However, the soil parameters vary with frequency, and for the frequency dependent soil, the resistance is $1.98 \Omega$.

There is a very minimal reduction in resistance value at $5 \mathrm{kHz}$, with frequency dependent soil parameters. It can be observed from Figure 5.13(b) that the phase angle changes from $3.2^{\circ}$ to $2.9^{\circ}$ with frequency dependency, indicating a reduction in the inductive component. This is due to the high relative permittivity at low frequencies, which increases the capacitive reactance. At higher frequencies of $1 \mathrm{MHZ}$ and $4.52 \mathrm{MHz}$, the impedance increases to $18.61 \Omega$ and $28.43 \Omega$. This increase in impedance has two causes: firstly, an increase in inductive reactance, and secondly, increased resistance because of the skin effect.

Figures $5.14 \& 5.15$ demonstrate the results for $1000 \Omega \mathrm{m}$ soil resistivity. According IEC 61400-24, the minimum electrode length required is $20 \mathrm{~m}$. The mean radius of the ring electrode is less than $20 \mathrm{~m}$ and hence different electrode arrangements are designed to fulfil the $20 \mathrm{~m}$ electrode length.

The first arrangement comprises two ring electrodes buried at different depths with both the rings interconnected. Next, two horizontal electrodes are implemented, each with an individual length of $20 \mathrm{~m}$ for $1000 \Omega \mathrm{m}$ soil resistivity. Then, two $10 \mathrm{~m}$ vertical electrodes are connected to the WTG earthing grid. In the next case, one ring electrode along with two horizontal electrodes are connected to the WTG grounding system. Finally, an earthing arrangement of a ring electrode and two vertical electrodes is implemented.

From the calculated impedance values, it is observed that an earthing system with two horizontal electrodes provides low resistance value at $5 \mathrm{kHz}$, with a value of $17.24 \Omega$ and $16.45 \Omega$ for frequency independent and dependent soil parameters, respectively. The electrode configuration with two ring electrodes provides low impedance at $1 \mathrm{MHz}$ and $4.52 \mathrm{MHz}$. The combinations of ring with horizontal and ring with vertical provides 


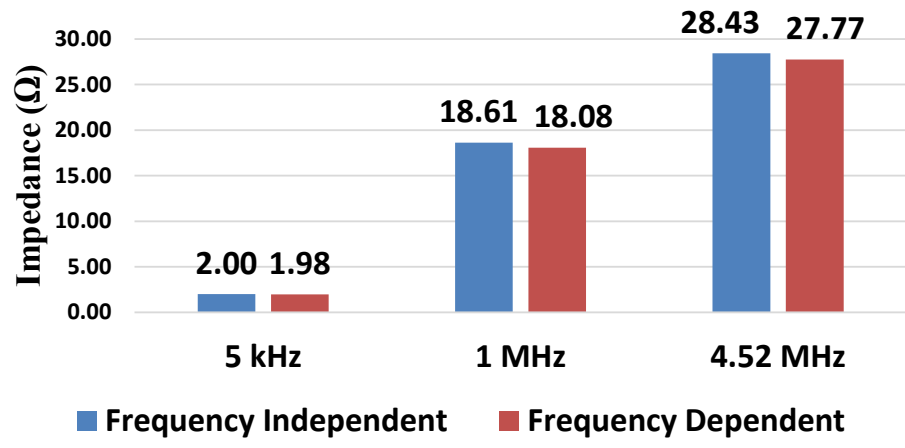

(a) Impedance.

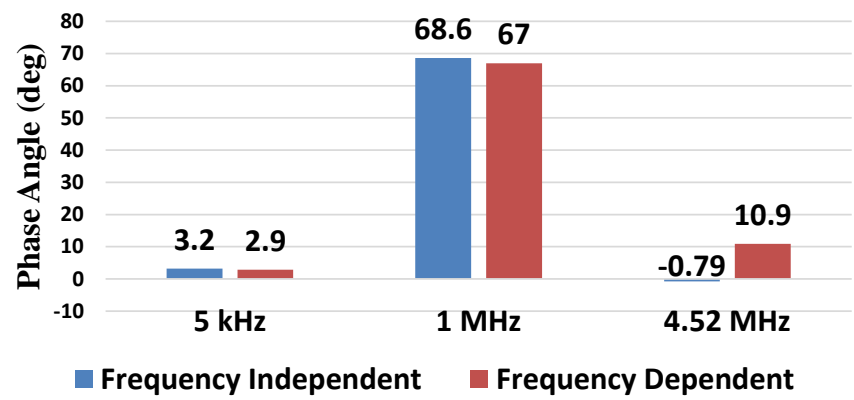

(b) Phase angle.

Figure 5.13: Impedance and phase angle of the WTG earthing system for $\rho=100 \Omega \mathrm{m}$. 


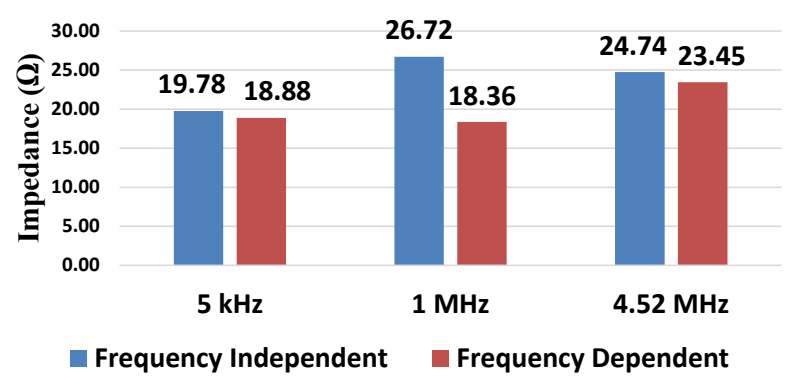

(a) Impedance.

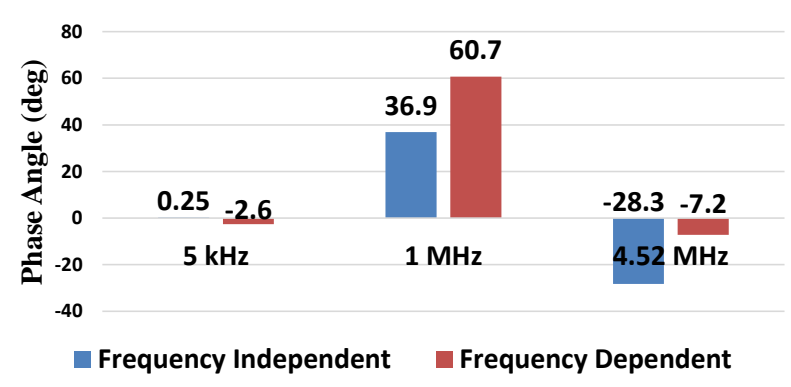

(b) Phase angle.

Figure 5.14: Impedance and phase angle of the WTG earthing system with ring electrodes for $\rho=1000 \Omega \mathrm{m}$. 
low impedance for all the frequencies of interest. Moreover, frequency dependent soil parameters provide a low impedance for all the cases except horizontal and vertical electrodes at $4.52 \mathrm{MHz}$.

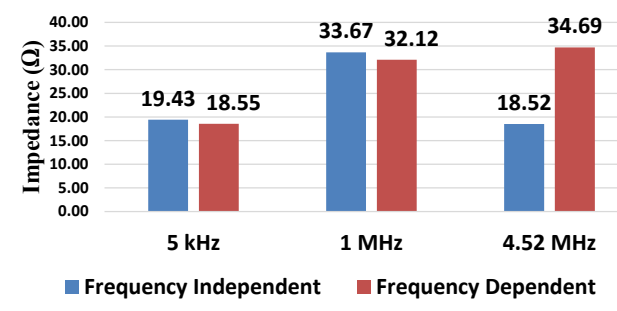

(a) Two vertical electrodes.

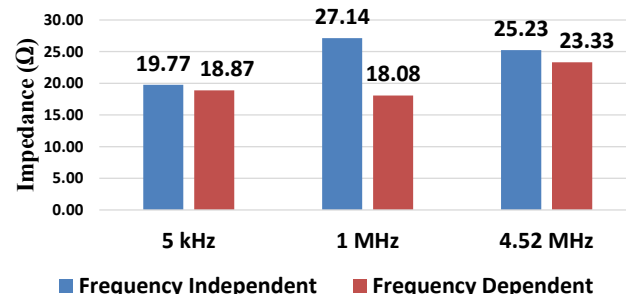

(c) Ring and vertical electrodes.

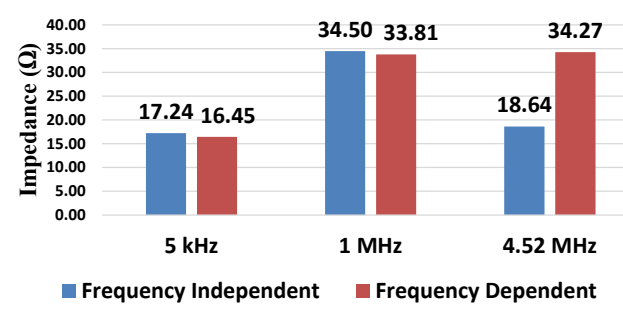

(b) Two horizontal electrodes.

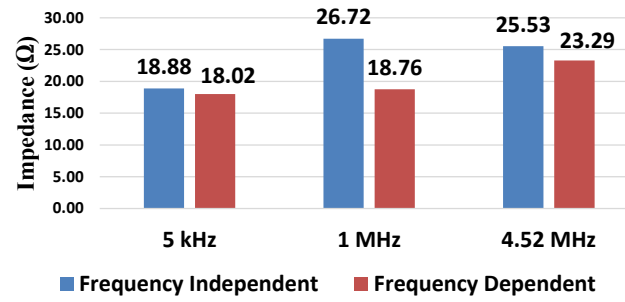

(d) Ring and horizontal electrodes.

Figure 5.15: Impedance of the WTG earthing system with different electrodes for $\rho=1000 \Omega \mathrm{m}$.

For soil resistivity of $2000 \Omega \mathrm{m}$ a minimum electrode length of $50 \mathrm{~m}$ is needed. Various electrode arrangements, depicted in Figures 5.4 \& 5.5, are designed for this case. The computed impedance and phase angles are depicted in Figures 5.16 to 5.18. However, none of the electrode configurations guarantees an impedance value of less than $10 \Omega$ for all the frequencies. An electrode configuration consisting of one ring electrode and two horizontal electrodes each of $200 \mathrm{~m}$ length is implemented to get $10 \Omega$ resistance at $5 \mathrm{kHz}$. However, the impedance value does not vary after a particular length for high frequencies.

Figures $5.19 \& 5.20$ show the results for the case of $10 \mathrm{k} \Omega \mathrm{m}$ soil resistivity. The impedance values are larger with frequency independent soil 


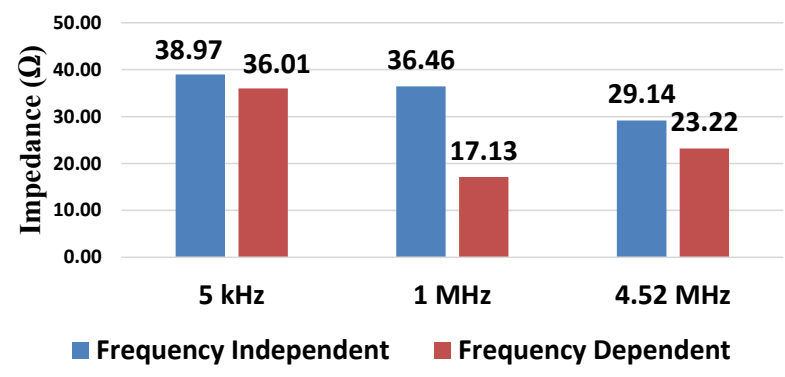

(a) Impedance.

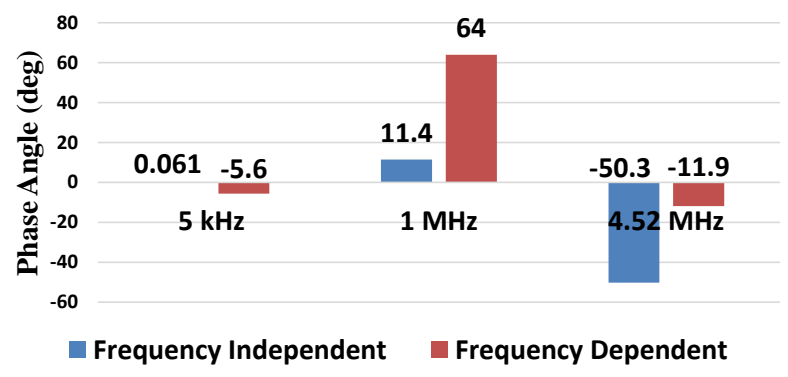

(b) Phase angle.

Figure 5.16: Impedance and phase angle of the WTG earthing system with five ring electrodes for $\rho=2000 \Omega \mathrm{m}$. 


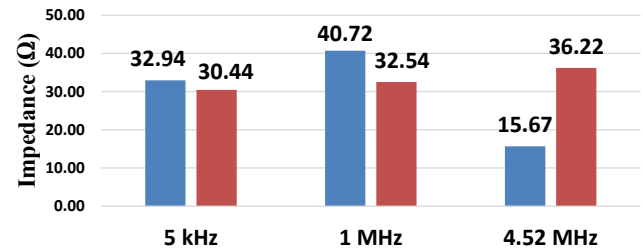

- Frequency Independent $\quad$ Frequency Dependent

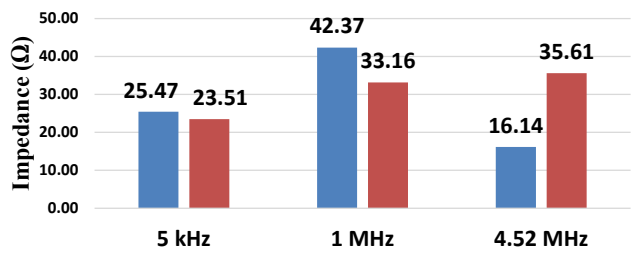

- Frequency Independent Frequency Dependent

(a) Two vertical electrodes $(25 \mathrm{~m})$.

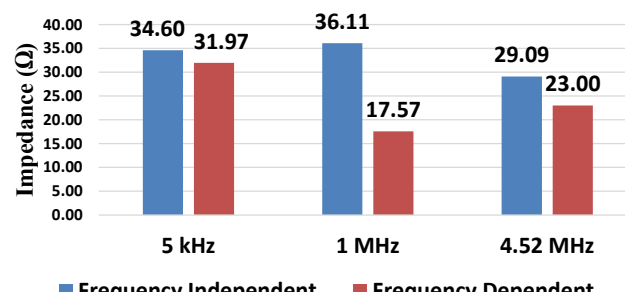

(c) Ring and vertical electrodes $(20 \mathrm{~m})$.

(b) Two horizontal electrodes (50 m).

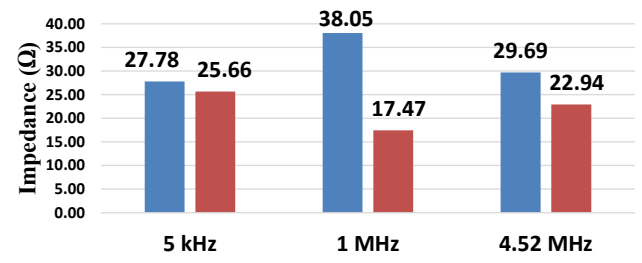

arequency Independent $\quad$ Frequency Dependent

(d) Ring and horizontal electrodes (40 m).

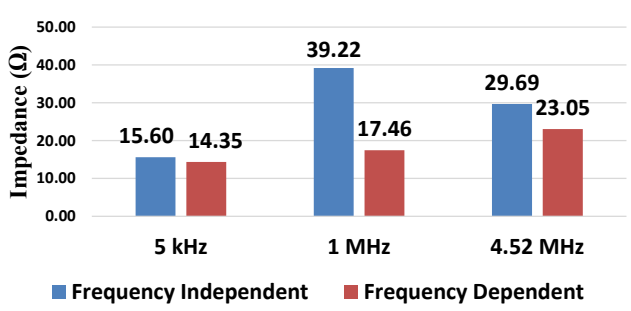

(e) Ring and horizontal electrodes (120 m).

Figure 5.17: Impedance of the WTG earthing system for different electrodes for $\rho=2000 \Omega \mathrm{m}$. 


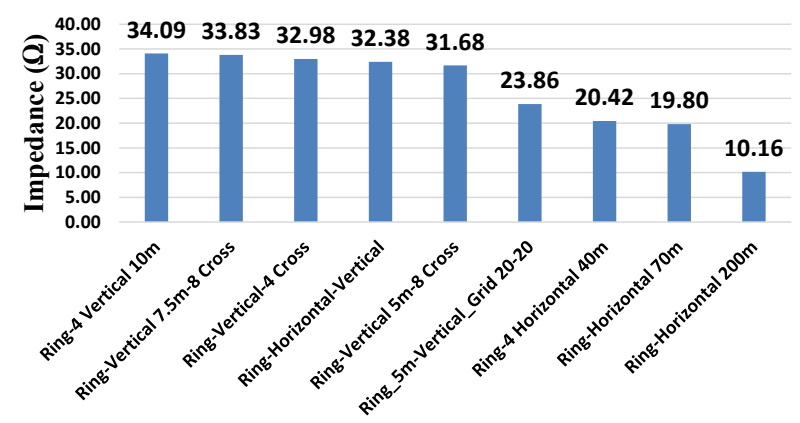

(a) $5 \mathrm{kHz}$.

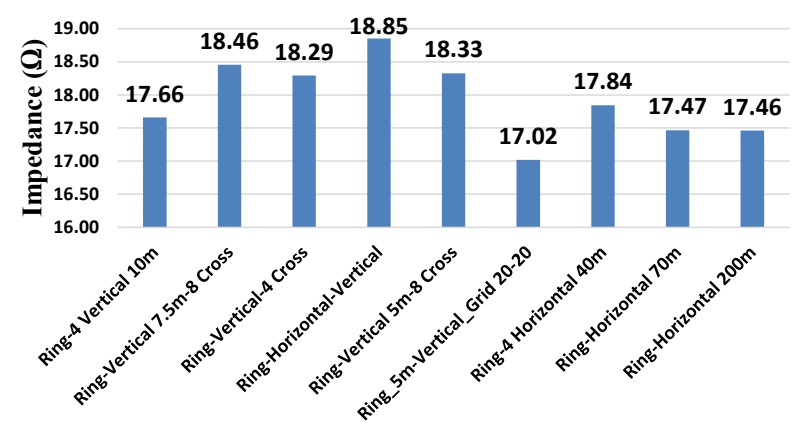

(b) $1 \mathrm{MHz}$.

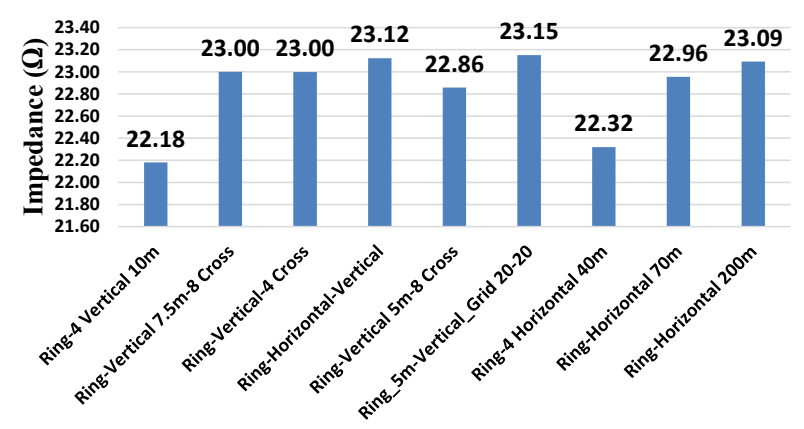

(c) $4.52 \mathrm{MHz}$.

Figure 5.18: Impedance of the WTG earthing system for $\rho=2000 \Omega \mathrm{m}$ for various electrode configurations. 
parameters reaching up to $138 \Omega$. However, with frequency dependent soil parameters, the impedance values are reduced drastically. The combination with ring and horizontal electrodes provides low impedance path of $78.74 \Omega, 15.44 \Omega$, and $23.30 \Omega$ at $5 \mathrm{kHz}, 1 \mathrm{MHz}$, and $4.25 \mathrm{MHz}$ respectively.

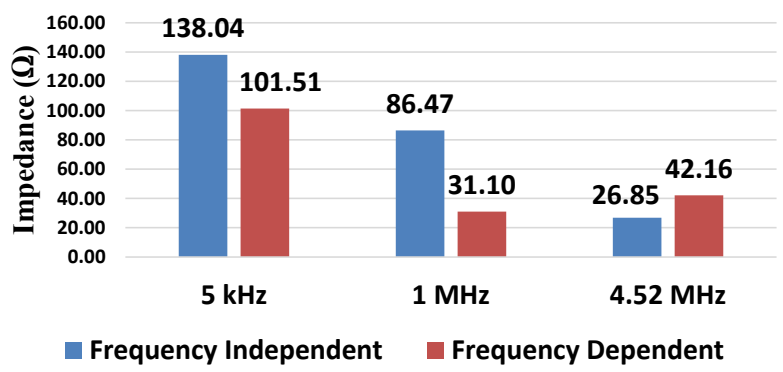

(a) Impedance.

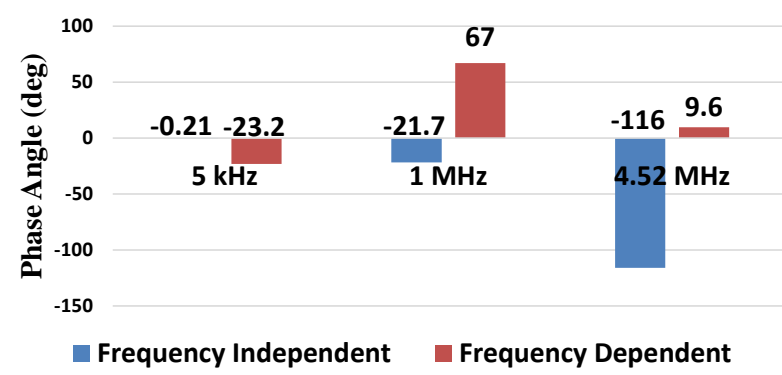

(b) Phase angle.

Figure 5.19: Impedance and phase angle of the WTG earthing system with vertical electrodes for $\rho=10 \mathrm{k} \Omega \mathrm{m}$.

This analysis shows that it is very difficult to achieve a low impedance of $10 \Omega$ at all frequencies. The electrode configuration with ring \& horizontal and ring \& vertical electrodes provide the lowest impedance amongst all the electrode configurations considered. However, the ring and vertical electrodes configuration is not practically feasible as it is difficult to drill deep bores for the vertical electrodes. For high soil resistivity sites, the grounding system with a ring electrode configuration is not feasible due to the requirement of multiple ring electrodes. For example, a WTG site with $3000 \Omega \mathrm{m}$ resistivity of the soil requires an electrode of length $80 \mathrm{~m}$. A WTG 


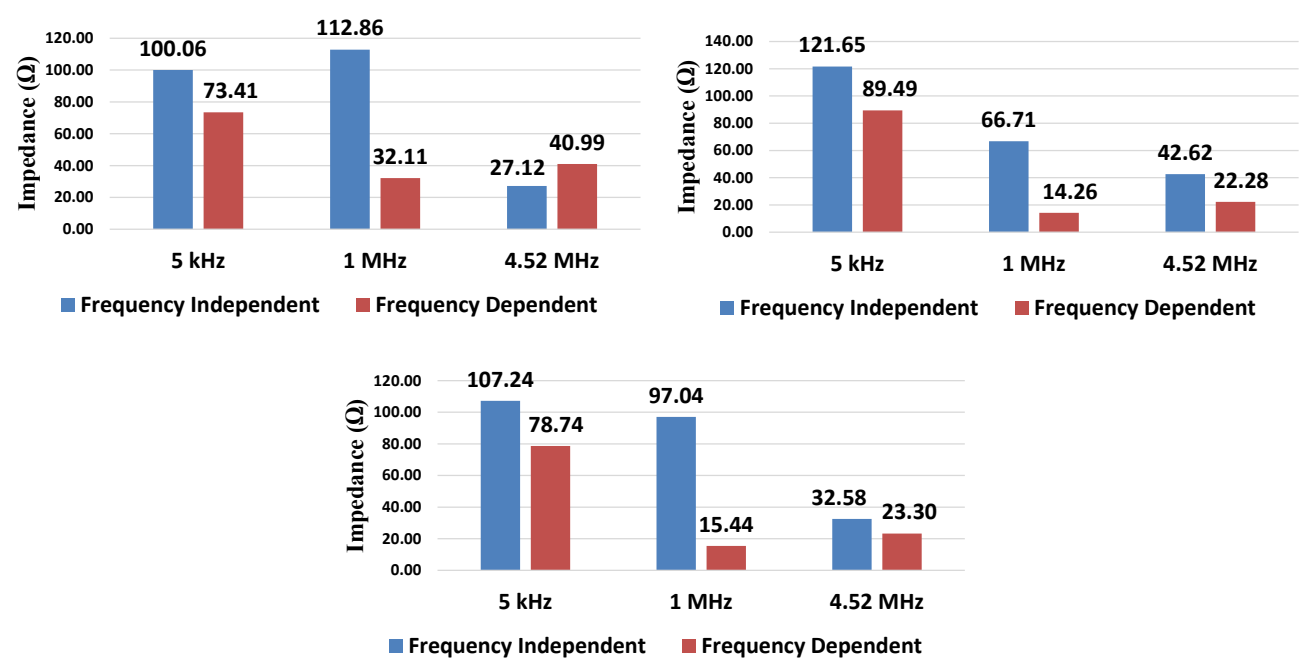

Figure 5.20: Impedance of the WTG earthing system with different electrodes for $\rho=10 \mathrm{k} \Omega \mathrm{m}$.

foundation with a radius of $10 \mathrm{~m}$ needs eight ring electrodes. The number ring electrodes must be installed either radially or vertically. However, electrical fields of the individual ring electrodes interfere with surrounding electrodes if not space properly. Hence, the ring electrodes should be installed in a way that should not interfere with the electric fields of the ring electrodes.

The analysis of WTG earthing impedance of various electrode configurations leads to particular consideration of selected electrodes for lowfrequency resistance and impulse coefficient analysis. As illustrated in Figure 5.21, the low-frequency resistance is lowest for the grounding system with horizontal electrodes. The next best electrode configuration is the ring-horizontal electrode. Besides, the low-frequency resistance is further reduced with increase in the length of the horizontal electrode, as observed for a combination of ring and $120 \mathrm{~m}$ horizontal electrode for $2000 \Omega \mathrm{m}$ soil resistivity. The low-frequency resistance of high soil resistivity sites is very high. This is mainly due to the increased resistivity and lack of electrode length to dissipate the lightning discharge currents. 


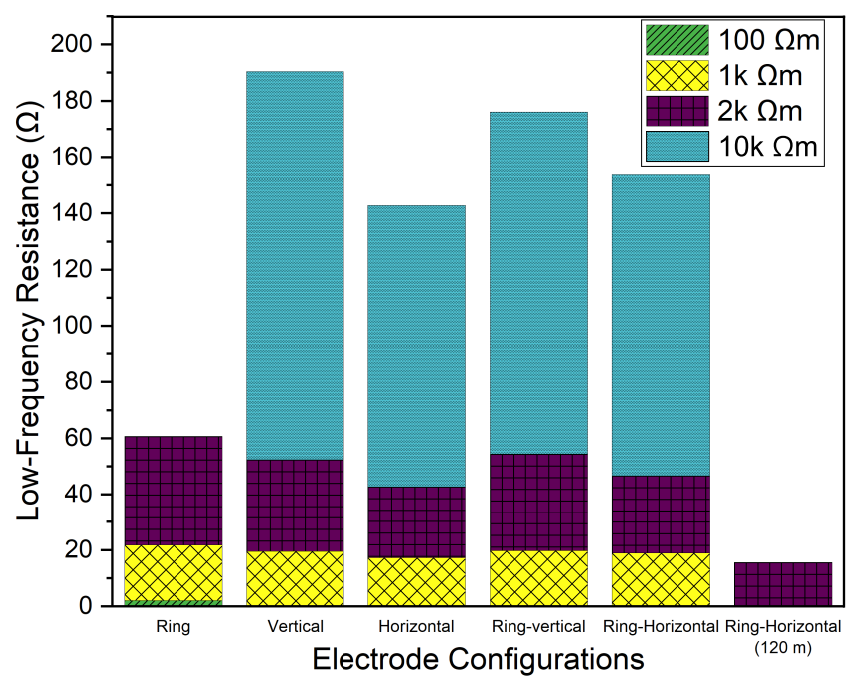

Figure 5.21: Comparison of low frequency resistance for various electrode configurations for uniform soil resistivities.

However, the impulse efficiency of the grounding system is different from the low-frequency performance. As depicted in Figure 5.22, the impulse coefficient at $100 \Omega \mathrm{m}$ is very high compared to high soil resistivity sites. This is due to the high impedance of the grounding system at higher frequencies and small values of low-frequency resistance. At higher soil resistivities, the ring electrode and its combination with other electrodes have better impulse coefficients due to the better performance of ring electrodes at high frequencies. The performance degradation of the horizontal electrode configuration from the impulse coefficient view point is mainly due to its better performance at lower frequencies.

\subsubsection{Effectiveness of the WTG earthing system for various soil mod- els}

In the final scenario, the effectiveness of the WTG grounding system for various soil models for different electrode configurations is compared. A 


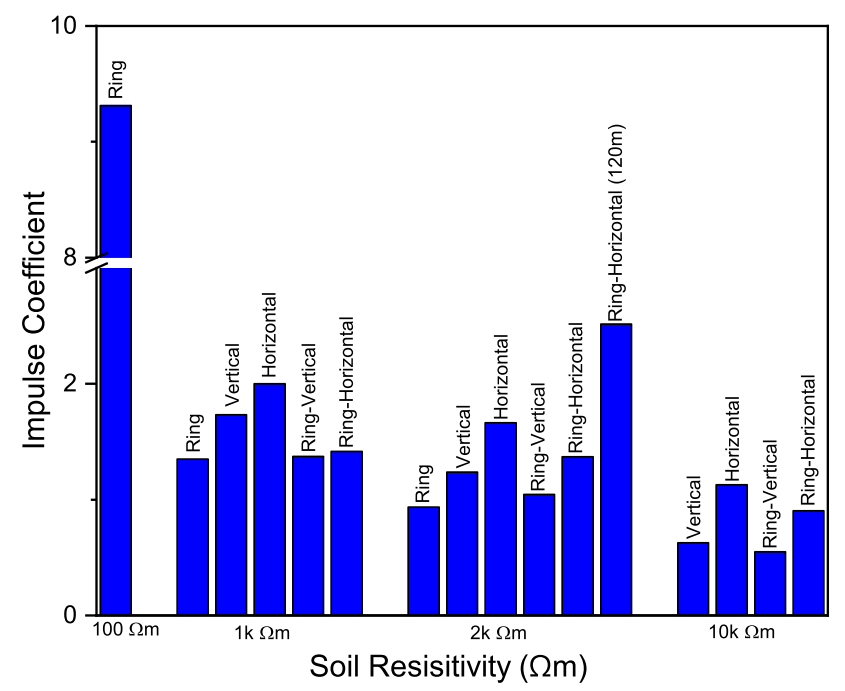

Figure 5.22: Comparison of impulse coefficient for various electrode configurations for various uniform soil resistivities.

set of measured soil resistivity values were modeled to various soil structures, viz. uniform, horizontal two-layer, and horizontal three-layer soil structures in [34] are used for this analysis. As shown in Figure 5.23, the low-frequency resistance is lowest for horizontal earth electrodes irrespective of the soil structure. Also, it is noted that the horizontal three-layer soil model has the least low-frequency resistance amongst all the soil models.

As illustrated in Figure 5.24, the impulse coefficient is best for the electrode configuration consisting of a ring electrode. This is due to the better performance of the ring electrodes at high frequencies. The impulse coefficients are better for the uniform soil model compared to horizontal two \& three layer soil structures. This is due to the performance impairment of the uniform soil model at low frequencies.

The potential gradient developed due to the lightning impulse current is smallest for horizontal electrodes for all the soil models at lowfrequencies. Moreover, the potential is lowest for the horizontal threelayer soil model amongst all the soil structures. The high-frequency po- 


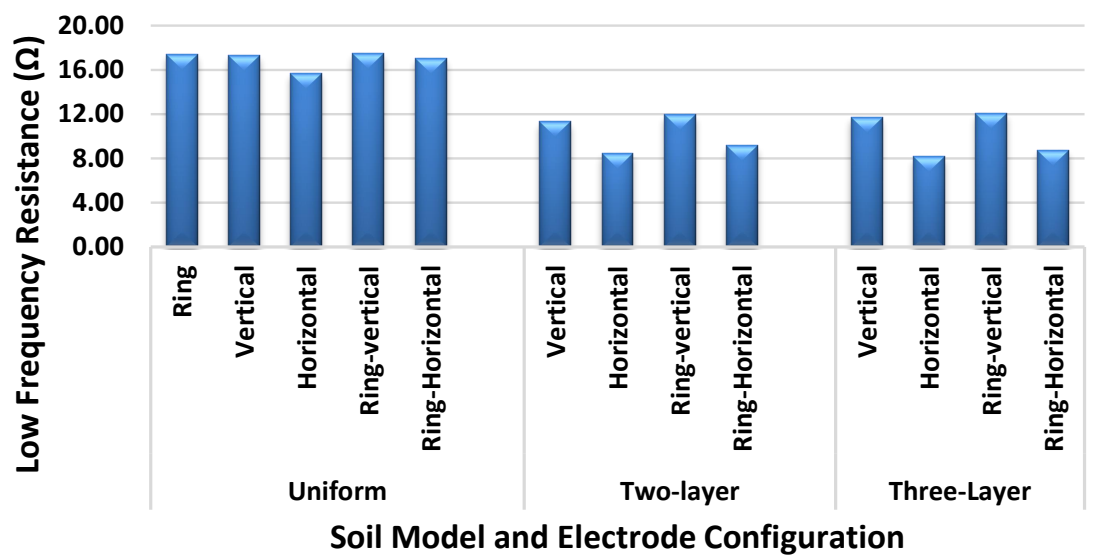

Figure 5.23: Comparison of low-frequency resistance for various electrode configurations with various soil models

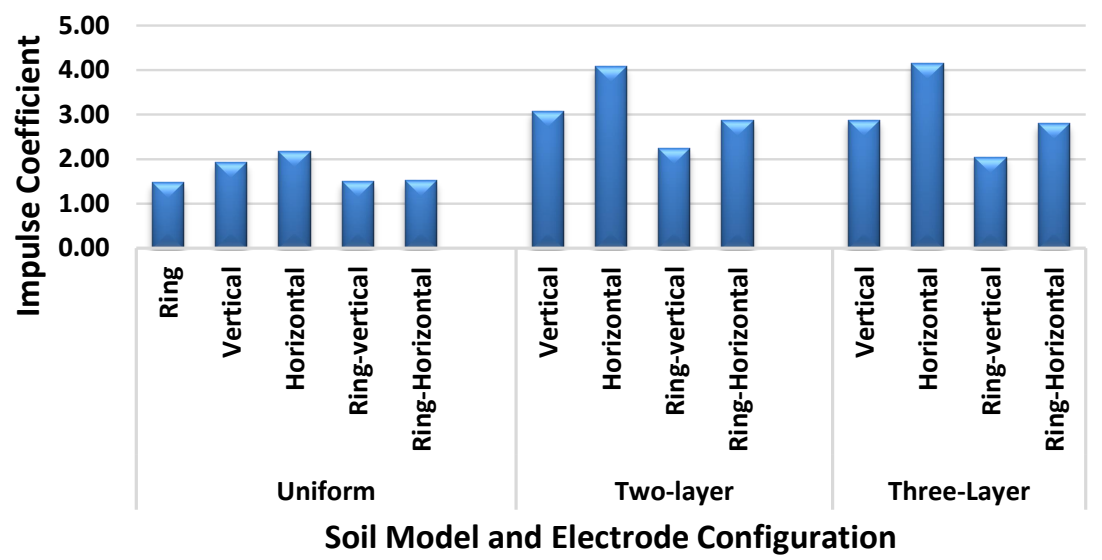

Figure 5.24: Comparison of impulse coefficient for various electrode configurations with various soil models and frequency dependent soil parameters. 
tential magnitude is minimum for the electrode configuration comprising of ring-horizontal electrode configuration, as depicted in Figure 5.25.

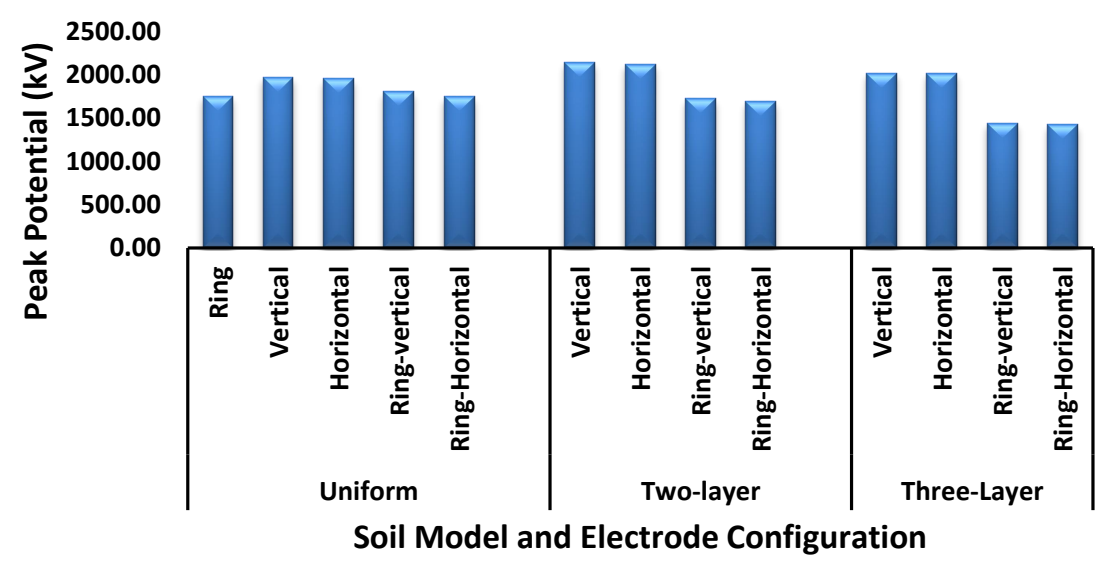

Figure 5.25: Peak potenial for various electrode configurations and soil models.

The step voltages have a minimum value for horizontal electrodes at low frequencies irrespective of the soil model. Also, a minimum amount of step voltage is observed for horizontal three-layer soil structure at low frequencies. However, at high frequencies, the electrode configuration of ring-horizontal electrodes offers the least step voltage, as illustrated in Figure 5.26 .

The horizontal electrode configuration provides a low resistance grounding system at low-frequencies due to the uniform electric field distribution leading to equipotential surface throughout the grounding grid. At higher frequencies the current dissipation is higher at the impulse injection point. Moreover, the potential rise at the ring electrodes is higher compared to other electrodes. The same phenomenon is observed regardless of soil structure or LPL. Hence, a ring electrode in combination with a horizontal electrode is the preferred electrode configuration for all soil structures and LPLs.

The results also show that the length of the horizontal electrode should 


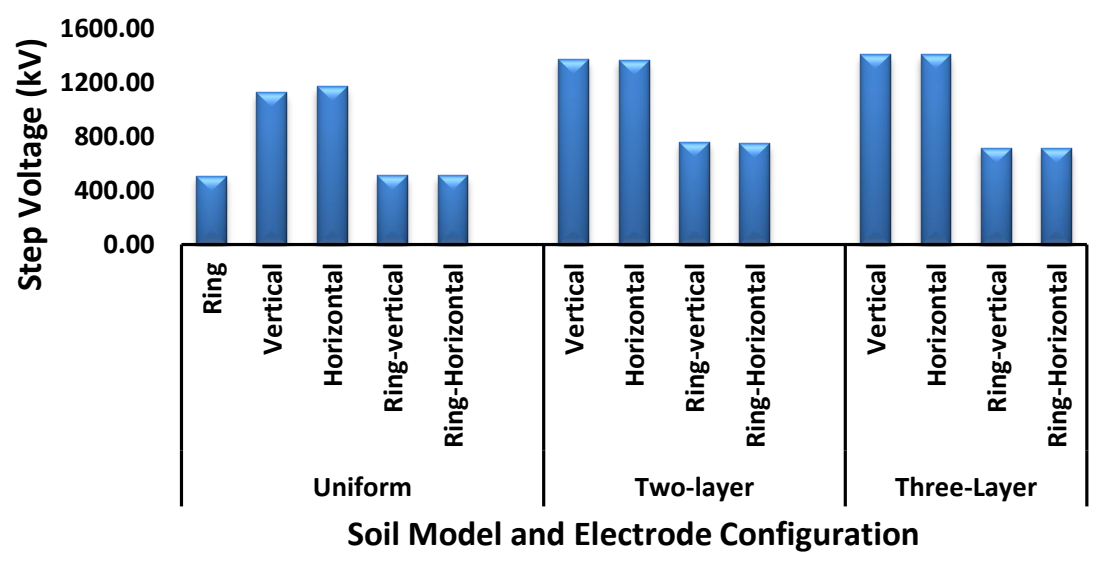

Figure 5.26: Step voltage for various electrode configurations and soil models.

be longer than recommended in IEC 61400-24. The calculated soil resistivity changes using frequency dependent soil parameters, which in turn reduces the wind turbine impedance at higher frequencies. This effect is strong for wind turbines installed at high soil resistivity locations, with a reduction of up to $85 \%$ at $1 \mathrm{MHz}$ for $10 \mathrm{k} \Omega \mathrm{m}$ soil resistivity. It can be concluded that WTG earthing impedance is over-estimated when the frequency dependent nature of the soil resistivity is ignored.

The effectiveness of a WTG lightning protection system depends mainly on two factors: the choice of WTG site and a well-designed grounding system. The assessment of WTG site for lightning probability and low soil resistivity is vital in the wind farm optimization for energy yield and safety. The WTG grounding system designed with an accurate soil resistivity measurement and soil modeling along with an electrode configuration of the ring and horizontal electrodes ensures an effective grounding system. The increase in the length of horizontal electrodes beyond an effective length does not affect the high-frequency impedance. However, it improves the low-frequency resistance, which is also the steady state impedance of the grounding system. 


\subsection{Chapter summary}

This chapter presented a procedure for assessing the effectiveness of WTG lightning protection system. The installation of WTGs at low probability of lightning incidence and the design of effective grounding systems are the parameters controllable by the wind farm designers. This chapter explains that the effectiveness of the grounding system can be improved by proper design of earth electrodes, soil stratification, and low resistivity soil sites. Following are the main contributions of this chapter:

1. This research provides a framework to evaluate the effectiveness of WTG LPS

2. The impulse coefficient is not the only parameter to assess the effectiveness of a LPS but to consider the low-frequency resistance and the potential distribution

3. The electrode configuration with ring and horizontal electrodes is the most effective for WTG LPS

4. Accurate soil stratification is important in effective LPS

5. The design of the WTG grounding system for LPL-1 parameters is recommended 


\section{Chapter 6}

\section{A Proposed Method for Calculating Earth Electrode Length for a Wind Turbine Generator Earthing System}

This chapter proposes a novel method to calculate the minimum length of an earth electrode for a WTG grounding system by considering the electrode dimensions and burial depth. A method to calculate the minimum length of an earth electrode for a WTG grounding system based on a twolayer soil model is also proposed. This methodology can be used to calculate the electrode length for a required value of grounding resistance. The proposed method is tested for various WTG sites and compared with the traditional practices.

\subsection{Introduction}

WTG grounding systems commonly serve two purposes: power system fault protection and lightning protection [137]. However, grounding sys- 
tem behaviour is different for lightning discharge currents compared to fault currents due to the high-frequency components present in lightning discharge currents [142]. At higher frequencies the reactive component of the impedance is dominant compared to the low-frequency resistance. Hence, protection of WTGs from lightning strikes takes priority over power system faults when designing WTG grounding systems.

The grounding grid impedance, potential gradients within a WTG earthing system, and the minimum electrode length required to achieve a resistance of less than $10 \Omega$ are dependent on the soil resistivity [17], [167]. Hence, the soil resistivity measurement and the interpretation of those values to determine the soil structure are critical in selecting the length of the earth electrode. In practice, soil resistivity varies with temperature, moisture, mineral content and compactness [173] as well as frequency. It is important to consider this frequency dependency while designing the earthing system owing to the high frequency components of the lightning discharge currents.

Another important factor in the design of a WTG grounding system is the soil structure, which is determined using soil models based on resistivity measurements. The current practice in WTG grounding design is to use a uniform soil resistivity model [17], [140]. The IEC 61400-24 standard assumes uniform soil resistivity, as depicted in Figure 2.10 of Section 2.5.3. However, soil resistivity in reality is not uniform, but varies both in the horizontal and vertical directions.

Although there are many soil models described in the literature, the most commonly used models in the industry are uniform and horizontal multi-layer [173]. A uniform soil model assigns a fixed resistivity value to the entire WTG foundation area. Soil that exhibits inhomogeneous properties can be modelled as horizontal multi-layers with varying layer depth and resistivity [173]. Hence, for the design of a WTG grounding system, soil is commonly modelled as a horizontal two-layer or three-layer structure, depending on the WTG site. 
Unfortunately, the existing literature lacks a method to design a WTG grounding system based on a horizontal multi-layer soil structure. Moreover, there are no recommendations made in the standard to calculate the electrode length for horizontal multi-layer soil models. The conventional methods do not consider the dimensions and burial depth of the earth electrodes while determining the length of the earth electrodes either. The conventional method of the grounding system design is based on the IEC 62305 standard designed originally for the lightning protection of buildings. The foundations of buildings have longer radius compared to WTG foundation and hence can have longer ring earth electrodes, which is a significant component of type-B earthing system. The adoption of this method for the WTGs need modifications, and this thesis aims to address some of the challenges.

\subsection{Soil Models}

The electrical potential distribution in soil depends on the electrical currents flowing into the soil through the earthing electrodes. These electrical currents and consequent potential variation are based on the resistivity of the soil [197]. Therefore, the modelling of the soil resistivity is critical for power system fault and lightning protection grounding system studies.

The techniques used for soil resistivity measurements are similar for most of the earthing system designs [195]. However, the interpretation of the measured data varies significantly, which is an important step in the soil modelling process. The interpretation of soil resistivity measurements essentially depends on the following factors [195]:

1. The accuracy and extent of the measurements

2. The method used

3. The complexity of the mathematics involved; and 
4. The purpose of the measurements

A uniform soil model has a single soil resistivity value for the entire earth mass with an infinite soil depth, as shown in Figure 6.1 [152].

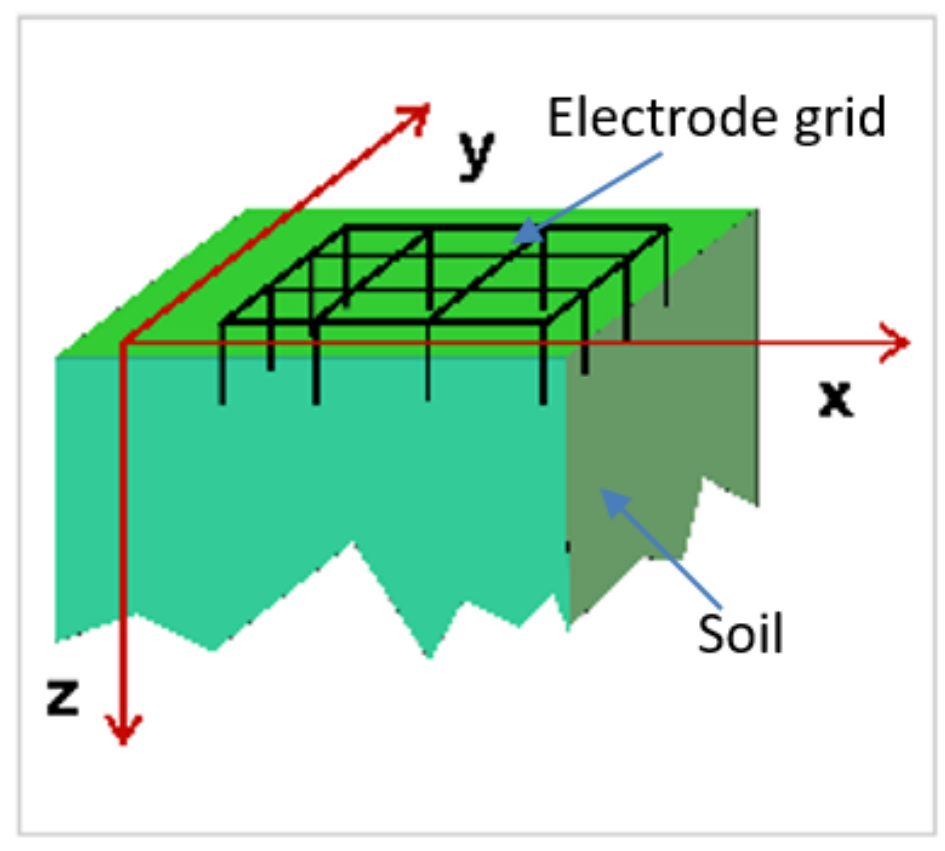

Figure 6.1: Uniform soil model [152].

A horizontal two-layer soil model depicted in Figure 6.2 [152], consists of two layers of different soil resistivity and a finite thickness of a top layer and the bottom layer extending to infinity. Similarly, a horizontal multilayer soil model consists of several layers with a varying soil resistivity for each layer, as depicted in 6.3 [198]. A more detailed description of soil models is discussed in Chapter 4.

\subsection{Equivalent soil resistivity model}

Using a multi-layer soil model is more realistic than a uniform soil model due to the inherent variation in soil structure and composition. How- 


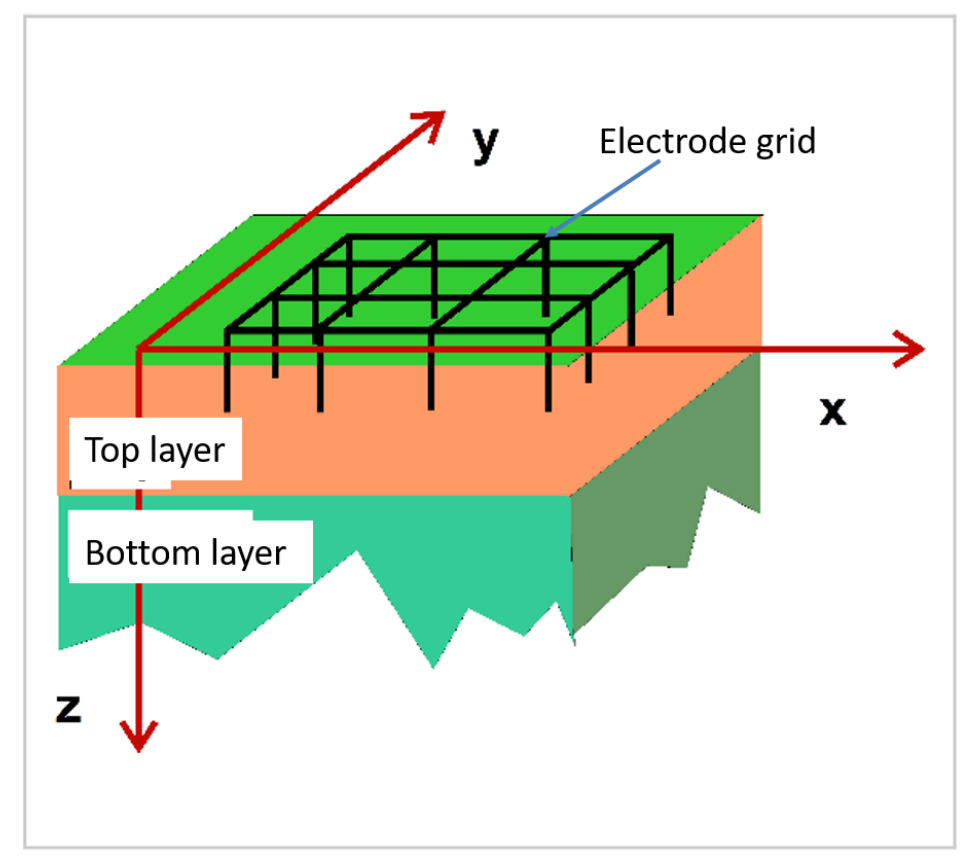

Figure 6.2: Horizontal two-layer soil model [152].

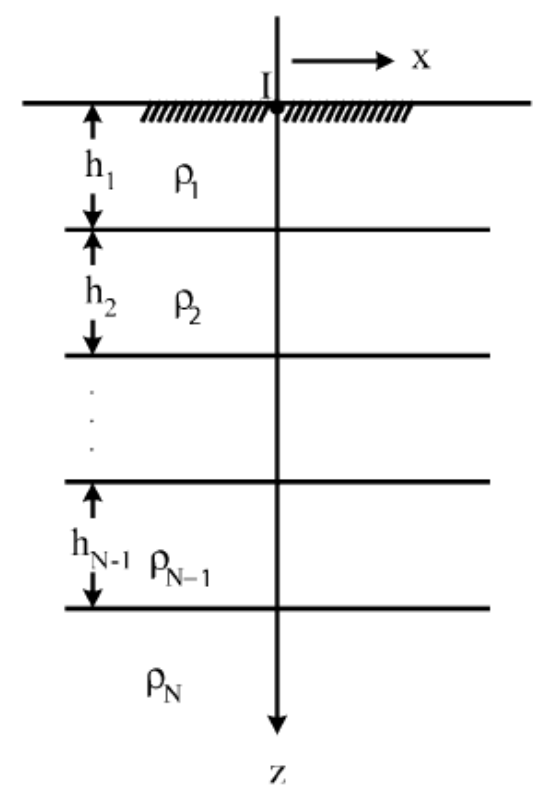

Figure 6.3: Horizontal multi-layer soil model [198]. 
ever, the validity of this representation is dependent on the accuracy of soil resistivity measurements [199]. Moreover, the soil resistivity varies significantly with temperature, moisture and the mineral content of the soil [173]. Therefore, it would be practical to approximate the equivalent soil resistivity of a two-layer soil model. Damitrios et al. [199] proposed an expression for the conductivity of soil based on a two-layer model, given by Equation (6.1):

$$
\rho_{e q}=\frac{1}{\sigma_{1}}\left[\frac{\left(\sqrt{\sigma_{1}}+\sqrt{\sigma_{2}}\right)+\left(\sqrt{\sigma_{1}}-\sqrt{\sigma_{2}}\right) e^{-2 d \sqrt{\pi f \mu_{1} \sigma_{1}}}}{\left(\sqrt{\sigma_{1}}+\sqrt{\sigma_{2}}\right)-\left(\sqrt{\sigma_{1}}-\sqrt{\sigma_{2}}\right) e^{-2 d \sqrt{\pi f \mu_{1} \sigma_{1}}}}\right]^{2}
$$

where $\rho_{e q}$ is the equivalent soil resistivity, $\sigma_{1}$ is the conductivity of the first layer, $\sigma_{2}$ is the conductivity of second layer, $\mu_{1}$ is the permeability of first layer, $d$ is the thickness of the first layer and $f$ is the frequency.

\subsection{Proposed method}

\subsubsection{Proposed Method for Calculating Earth Electrode Length using a Uniform soil Resistivity Model}

The required low resistance of a WTG earthing system can be achieved by an accurate design of the length, diameter and burial depth of earthing electrodes [17]. The conventional earthing system recommended by IEC 61400-24 is designed initially for buildings having long foundations where longer ring electrodes are possible [200]. However, WTGs typically have foundations with shorter mean radius and might need additional electrodes for higher values of soil resistivity.

The mean radius of the ring earth electrode $\left(r_{e}\right)$ must be higher than the minimum length of the electrode $l_{1}$ based on Figure 2.10. However, if the required length of the electrode is greater than the radius of the ring electrode $(r)$, additional horizontal and vertical electrodes are connected. The required length of these additional electrodes can be calculated from 
Equations in (6.2) and (6.3) [17].

$$
\begin{gathered}
l_{r}=l_{1}-r_{e} \\
l_{v}=\frac{\left(l_{1}-r_{e}\right)}{2}
\end{gathered}
$$

where $l_{1}$ is the minimum length of the earth electrode, $l_{r}$ is the length of the radial electrode, $l_{v}$ is the length of the vertical electrode, and $r_{e}$ is the mean radius of the ring earth electrode.

In the conventional approach based on the IEC 61400-24 standard, the length of the earth electrode is dependent only on the soil resistivity; however, the current dissipation in an earthing system is dependent on the dimensions and the burial depth of the earth electrodes. Hence, the electrode dimensions and its burial depth are critical to the performance of a WTG earthing system.

The calculation of the ring electrode resistance is important in deriving the formula for the WTG earth electrodes. As depicted in Figure 6.4, the centre of the ring electrode is assumed to be at the origin of the coordinates. The resistance of a ring electrode can be calculated using Equa-

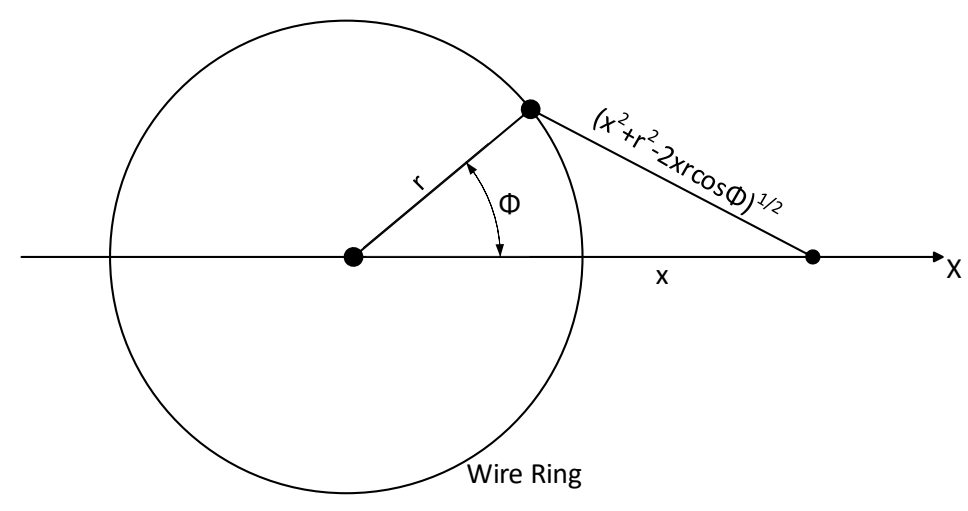

Figure 6.4: Coordinates for buried ring of wire [146].

tion (6.4), proposed by Sunde [146]. However, the calculation of resistance using this formula does not consider the structural foundation of 
the WTG. The WTG foundation consists of metallic components which can share the lightning discharge currents when connected to the grounding system.

$$
R=\frac{\rho}{2 \pi^{2} r_{e}} \ln \frac{8 r_{e}}{\sqrt{2 a d}}
$$

here, $a$ is the radius of cross section of the ring earth electrode, $d$ is the burial depth of the ring, $r_{e}$ is the mean radius of the ring, $R$ is the equivalent earth resistance of the ring, and $\rho$ is the soil resistivity.

Yasuda et al. [168] proposed a modified formula for calculating the WTG earthing resistance, given by Equation (6.5):

$$
R^{\prime}=\frac{\rho}{2 \alpha \pi^{2} r_{e}} \ln \frac{8 \beta r_{e}}{\sqrt{2 a d}}
$$

where $R^{\prime}$ is the equivalent earth resistance of WTG, and $\alpha$ and $\beta$ are improvement constants, determined using the method described in [168].

The proposed method is based on the equivalent resistance of the WTG earthing system given by (6.5). The mean radius of the ring earth electrode can be evaluated by rearranging Equation (6.5), to give Equation (6.6).

$$
r_{e}=-0.5 \frac{\rho}{R^{\prime} \alpha \pi^{2}} W\left(-0.3535533906 \frac{R^{\prime} \alpha \pi^{2} \sqrt{a d}}{\rho \beta}\right)
$$

here $W$ is the lambart $\mathrm{W}$ function.

Finally, the length of the ring electrode is evaluated by $l=2 \pi r_{e}$. It is important to note that the calculated length of the ring earth electrode may not be possible to implement for all WTG earthing systems because of the fixed size of the WTG foundation. We denote the radius of this foundation $r_{f}$, and the equivalent length of an electrode having this radius to be $x=2 \pi r_{f}$, with $x<l$. In such cases, the shortfall of the electrode length is made up by proposed radial or vertical electrodes according to Equations (6.7) and (6.8).

$$
\begin{aligned}
& l_{r p}=l-x \\
& l_{v p}=\frac{l-x}{2}
\end{aligned}
$$


where $l_{r p}$ is the proposed length of the horizontal electrode, $l_{v p}$ is the proposed length of the vertical electrode, and $x$ is the equivalent length of the WTG ring electrode.

\subsubsection{Proposed method for calculating the length of the earth electrode for a horizontal two-layer soil resis- tivity model}

The selection of the minimum length of the earth electrode for a horizontal two-layer soil model is ambiguous as the resistivity of the soil is different for the two layers. The foundation and the earthing system of a WTG can extend to the bottom layer of the earth. Moreover, the variation in resistivity of the soil is observed to be significant, which might lead to overestimation or underestimation of the electrode length. Besides, there is a strong dependency on the layer influence on the earthing system [195]. Hence, there is a need for a procedure to calculate the electrode length based on a multi-layer soil structure.

This research introduces a new methodology to calculate the electrode length for a horizontal two-layer soil structure. The proposed method is illustrated in the flowchart depicted in Figure 6.5:

The method starts with the measurement of soil resistivity at the intended WTG installation site. The site modelled as a horizontal two-layer soil structure using the measured soil resistivity values. The next step is to calculate the equivalent soil resistivity of the two-layer soil structure using Equation (6.1). The required mean radius of the ring electrode is calculated by Equation (6.6), which is derived from the formula for equivalent earth resistance of the WTG proposed by Yasuda et al. [168].

If the required mean radius of the electrode is greater than the actual radius of the WTG ground electrode $\left(r_{f}\right)$, additional horizontal or vertical electrodes are designed to meet the requirement. If the additional electrodes are required, then the minimum number of horizontal or vertical 


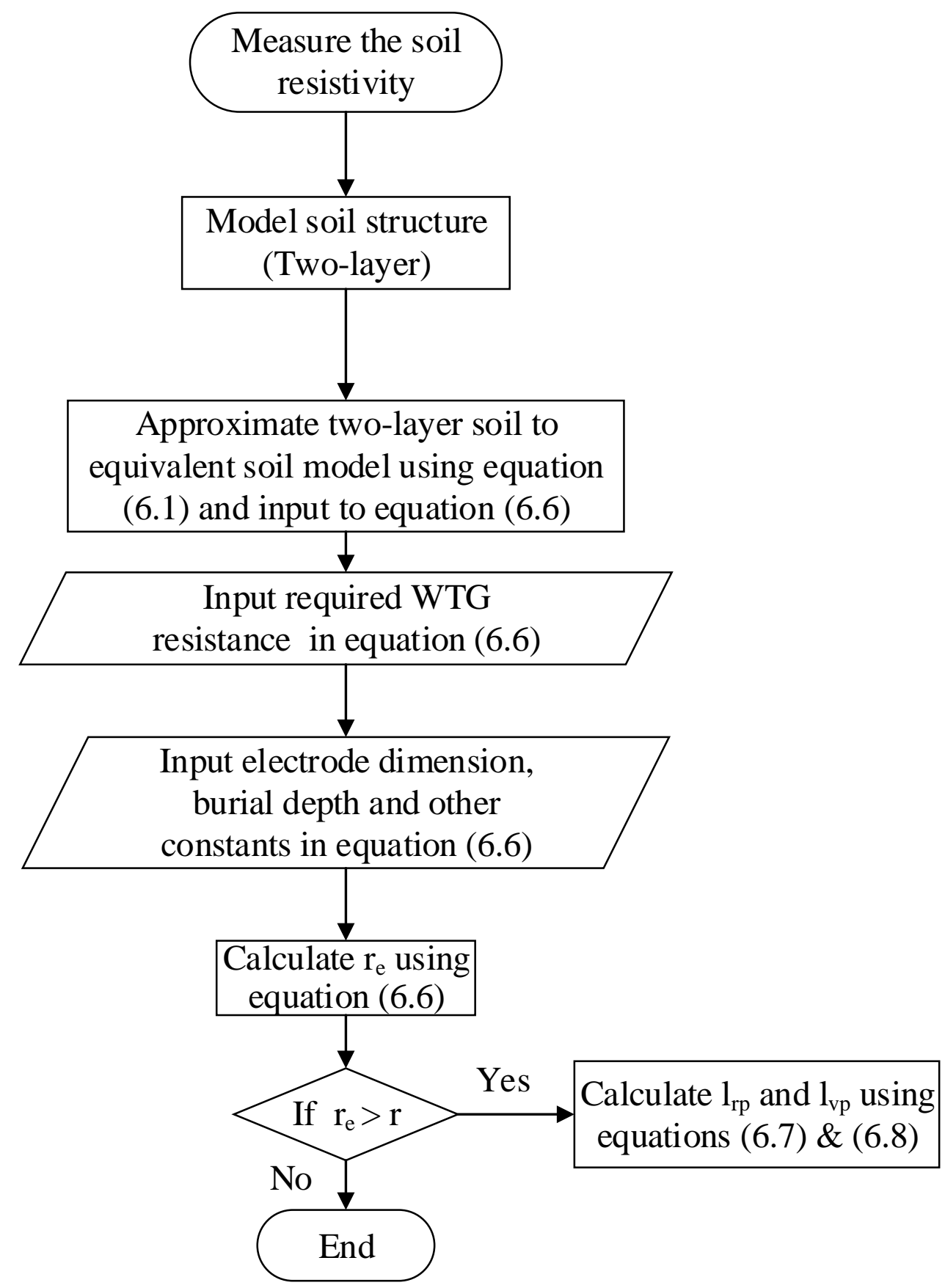

Figure 6.5: Proposed method for calculating earth electrode length for two-layer soil structure. 
electrodes should not be less than two [17]. The resistance of a ring electrode in the form of a square is slightly more than the straight wire electrode [146]. Hence, the equivalent length of the ring electrode should be converted to a straight wire for designing the horizontal and vertical electrodes. Finally, the length of the additional radial and vertical electrodes are calculated using Equations 6.7 and 6.8.

\subsection{Results and discussion}

Simulations are performed using the RESAP, FFTSES and HIFREQ modules of the CDEGS (Current Distribution, Electromagnetic fields, Grounding and Soil structure analysis) software package [152]. The RESAP mod-

ule is utilised to model the soil, FFTSES is used to obtain the frequency spectrum of the time domain lightning discharge current wave form, and the HIFREQ module is employed to calculate the ground potential rise at different frequencies of interest.

The transient behaviour of the WTG earthing system subjected to lightning strikes is investigated using the proposed method of designing earth electrodes. The effectiveness of the earthing system is determined by the current dissipating capacity of the grounding system. The design of the grounding electrodes dictate the behaviour of the grounding system. Based on Figure 2.10, an empirical formula given by Equation 6.9 can be deduced for calculating the minimum length of the earth electrode for lightning protection level-1. However, it can be observed from Equation 6.9 that the design of the minimum length of the earth electrodes is only dependent on the resistivity of the soil.

$$
l_{1}=\max \left(\frac{-1000+3 \times \rho}{100}, 5 m\right)
$$

where $l_{1}$ is the minimum length of the earth electrode in $\mathrm{m}$, and $\rho$ is the soil resistivity in $\Omega \mathrm{m}$. 
This research considers the required minimum value of the resistance, dimensions of the earth electrode and the burial depth of the electrode in calculating the minimum length of the electrode. Table 6.1 depicts the mean radius of the ring earth electrode to achieve the WTG resistance values of $10 \Omega$ and $5 \Omega$ based on the calculations performed using the proposed method. The electrode lengths for burial depths of $0.5 \mathrm{~m}$ and $2 \mathrm{~m}$ and soil resistivity values of $500 \Omega \mathrm{m}, 1500 \Omega \mathrm{m}$ and $3000 \Omega \mathrm{m}$ are shown in Table 6.1. It can be observed that, to achieve a resistance of $10 \Omega$ with a soil resistivity of $500 \Omega \mathrm{m}$ and an electrode depth of $0.5 \mathrm{~m}$, the minimum length of electrode required is $11.84 \mathrm{~m}$. Whereas an electrode length of $87.51 \mathrm{~m}$ is required when the resistivity of the soil is $3000 \Omega \mathrm{m}$. By changing the electrode burial depth to $2 \mathrm{~m}$, the required length of the electrode drops slightly to $10.76 \mathrm{~m}$ and $81.2 \mathrm{~m}$ for $500 \Omega \mathrm{m}$ and $3000 \Omega \mathrm{m}$, respectively. To achieve the WTG resistance of $5 \Omega$, the minimum electrode lengths have increased about two folds, as illustrated in Table 6.1.

Table 6.1: Mean radius of earth electrode $r_{e}(\mathrm{~m})$.

\begin{tabular}{|c|c|c|c|}
\hline Resistance $(\Omega)$ & $\begin{array}{c}\text { Electrode Burying } \\
\text { Depth }(\mathrm{m})\end{array}$ & $\begin{array}{c}\text { Soil Resistivity } \\
(\Omega \mathrm{m})\end{array}$ & $\begin{array}{c}\text { Mean Radius of } \\
\text { Ring Electrode }(\mathrm{m})\end{array}$ \\
\hline 10 & 0.5 & 500 & 11.847 \\
\hline 10 & 0.5 & 1500 & 40.6005 \\
\hline 10 & 0.5 & 3000 & 87.5101 \\
\hline 10 & 2 & 500 & 10.7671 \\
\hline 10 & 2 & 1500 & 37.418 \\
\hline 10 & 2 & 3000 & 81.201 \\
\hline 5 & 0.5 & 500 & 25.8284 \\
\hline 5 & 0.5 & 1500 & 87.5101 \\
\hline 5 & 0.5 & 3000 & 187.545 \\
\hline 5 & 2 & 500 & 23.6941 \\
\hline 5 & 2 & 1500 & 81.201 \\
\hline 5 & 2 & 3000 & 175.02 \\
\hline
\end{tabular}




\subsubsection{Frequency dependency of electrode length}

The importance of the frequency dependency of the resistivity of the soil is depicted in Figure 6.6. The increases in frequency decreases the effective resistivity of the soil. The resistivity of the soil drops to about $325 \Omega \mathrm{m}$ at $4 \mathrm{MHz}$ from $3000 \Omega \mathrm{m}$ at $100 \mathrm{~Hz}$. Hence the effective length of the earth electrodes required at higher frequencies reduces significantly.

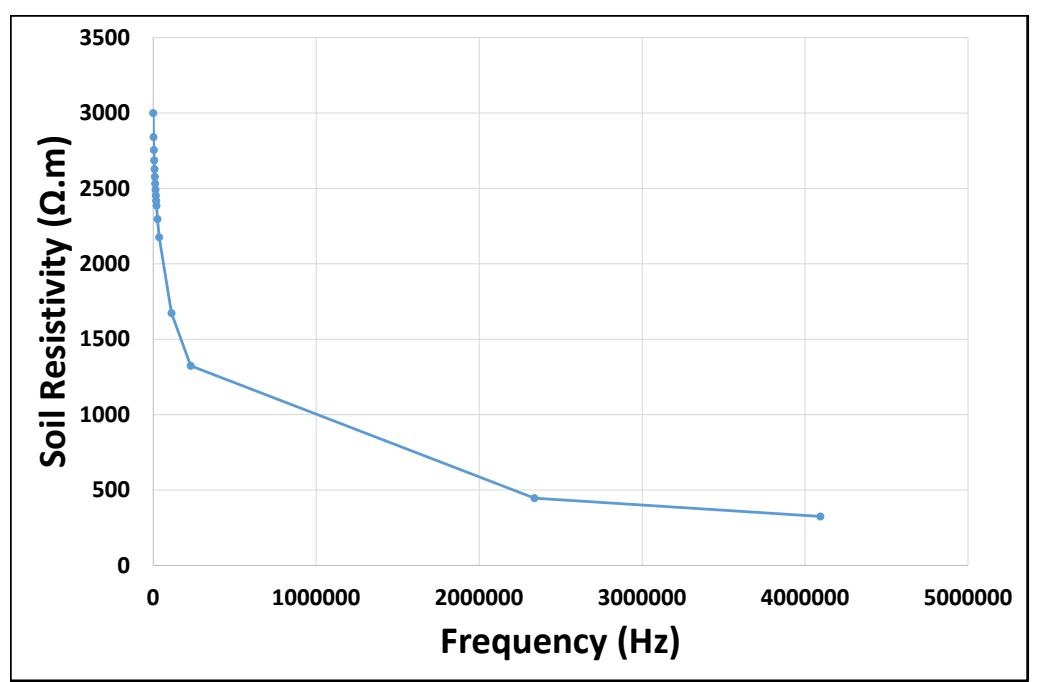

Figure 6.6: Frequency dependent soil resistivity evaluated based on the frequency-dependent soil parameters equations.

The WTG grounding electrode lengths calculated according to the standard IEC 61400-24 for frequency independent and dependent soil resistivity are depicted in Figure 6.7. For the resistivity values of the soil up to $500 \Omega \mathrm{m}$, an electrode length of $5 \mathrm{~m}$ is recommended by the standard method. Based on our studies, it is noted that the resistivity of the soil reduces to $60 \Omega \mathrm{m}$ and $150 \Omega \mathrm{m}$ at $4 \mathrm{MHz}$ for $100 \Omega \mathrm{m}$ and $500 \Omega \mathrm{m}$ at $100 \mathrm{~Hz}$, and hence an electrode length of $5 \mathrm{~m}$ is valid for the soil resistivities under $500 \Omega \mathrm{m}$. However, for a soil resistivity of $3000 \Omega \mathrm{m}$ at $100 \mathrm{~Hz}$ an electrode 
length of $80 \mathrm{~m}$ is required, whereas at $4 \mathrm{MHz}$ the soil resistivity is about $325 \Omega \mathrm{m}$, thus, an effective electrode length of $5 \mathrm{~m}$ is found to be sufficient. A similar pattern is observed for the other soil resistivity values ranging from $100 \Omega \mathrm{m}$ to $3000 \Omega \mathrm{m}$. The soil resistivity value drops below $500 \Omega \mathrm{m}$ at $4 \mathrm{MHz}$ for all the resistivity values from $100 \Omega \mathrm{m}$ to $3000 \Omega \mathrm{m}$, hence, an effective length of $5 \mathrm{~m}$ is sufficient at higher frequencies. For an earth resistivity of $10 \mathrm{k} \Omega \mathrm{m}$ at $100 \mathrm{~Hz}$ an electrode length of $290 \mathrm{~m}$ is required, however, the effective electrode length of $5 \mathrm{~m}$ is sufficient at $4 \mathrm{MHz}$ as the soil resistivity drops to $487 \Omega \mathrm{m}$.

The proposed method is applied to calculate the electrode lengths for two resistance values and different electrode parameters where the variation in electrode lengths are significant in some cases compared to the traditional method. Figure 6.8 shows the calculated electrode lengths for achieving a resistance of $10 \Omega$ when an electrode is assumed to be buried at a depth of $0.5 \mathrm{~m}$ below the earth surface. As depicted in Figure 6.8(a), an electrode length of $1.8 \mathrm{~m}$ is required for soil resistivity of $100 \Omega \mathrm{m}$ at $100 \mathrm{~Hz}$. As the soil resistivity reduces with an increase in frequency, an electrode length of $0.95 \mathrm{~m}$ is found to be sufficient at $4 \mathrm{MHz}$. For soil resistivity of $500 \Omega \mathrm{m}$, an electrode length of $11.8 \mathrm{~m}$ is required to achieve a resistance value of $10 \Omega$ at $100 \mathrm{~Hz}$, as illustrated in Figure 6.8(b), in contrast to a $5 \mathrm{~m}$ electrode length used in the conventional method. Also, at $4 \mathrm{MHz}$, an active electrode length of $3 \mathrm{~m}$ is required for the earth resistivity of $500 \Omega \mathrm{m}$. For soil resistivity of $3000 \Omega \mathrm{m}$, an electrode length of $87.5 \mathrm{~m}$ is required at $100 \mathrm{~Hz}$ and $7 \mathrm{~m}$ at $4 \mathrm{MHz}$ compared to a length of $80 \mathrm{~m}$ for all frequencies using the conventional method.

In the next scenario, the burial depth of the electrodes is changed to $2 \mathrm{~m}$ from the surface of the earth for calculating electrode lengths, as depicted in Figure 6.9. It can be seen that the required electrode lengths have reduced for all resistivity values of the soil when burying the electrodes deeper into the earth. The required length of the electrode is reduced from $87.5 \mathrm{~m}$ to $81.2 \mathrm{~m}$ at $100 \mathrm{~Hz}$ when the electrode depth is changed from $0.5 \mathrm{~m}$ 


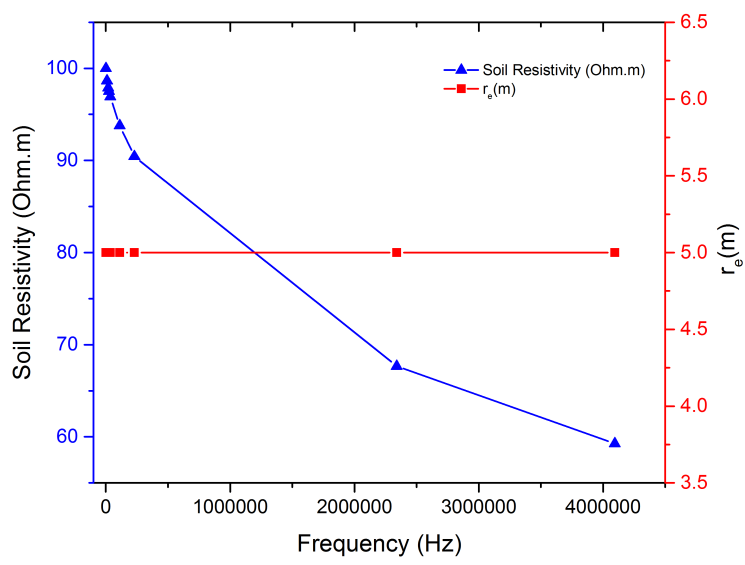

(a) $100 \Omega \mathrm{m}$

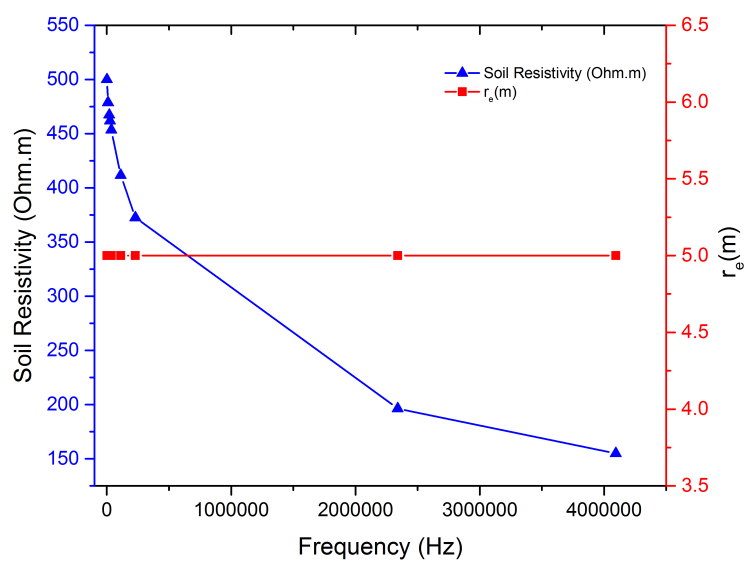

(b) $500 \Omega \mathrm{m}$

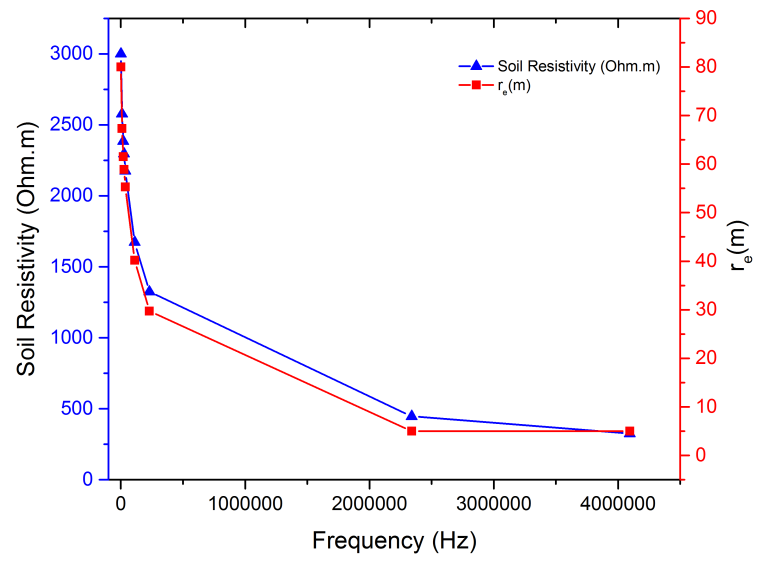

(c) $3000 \Omega \mathrm{m}$

Figure 6.7: Frequency dependent WTG earth electrode length according to IEC 61400-24. 


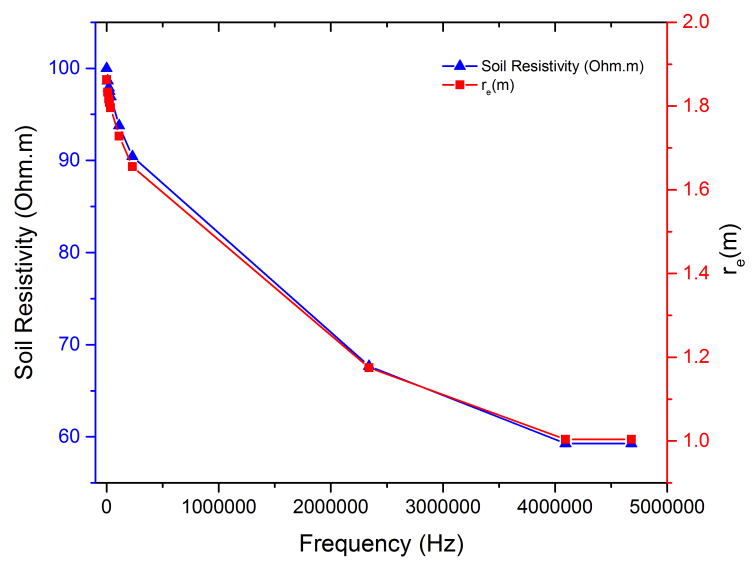

(a) $100 \Omega \mathrm{m}$

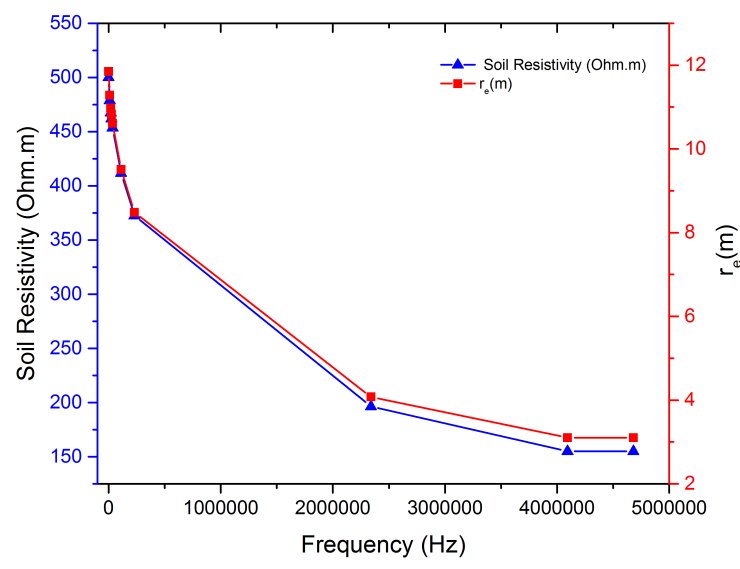

(b) $500 \Omega \mathrm{m}$

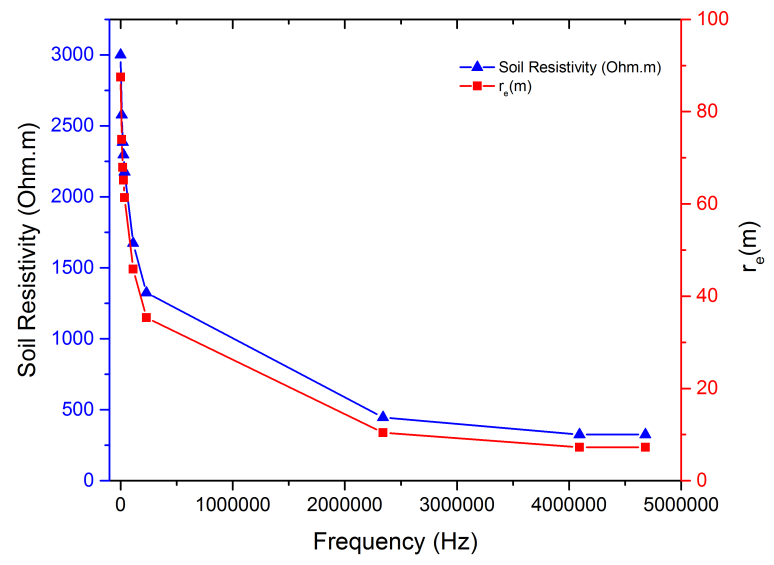

(c) $3000 \Omega \mathrm{m}$

Figure 6.8: WTG earth electrode length for $10 \Omega$ resistance for $0.5 \mathrm{~m}$ buried depth with the proposed method. 
to $2 \mathrm{~m}$. This is due to a more uniform electrical field distribution that exists around the electrodes.

In the next case, the electrode lengths are evaluated for achieving a resistance of $5 \Omega$. As illustrated in Figure 6.10, at a frequency of $100 \mathrm{~Hz}$, an electrode length of $4.1 \mathrm{~m}$ is required for $100 \Omega \mathrm{m}$, and an electrode length of $26 \mathrm{~m}$ is required at $500 \Omega \mathrm{m}$ against lengths of $1.8 \mathrm{~m}$ and $11.8 \mathrm{~m}$ for $10 \Omega$. For earth resistivity of $3000 \Omega \mathrm{m}$, an electrode length of $187.5 \mathrm{~m}$ is needed at $100 \mathrm{~Hz}$ and $16 \mathrm{~m}$ at $4 \mathrm{MHz}$.

In the final case, the electrode lengths are calculated to achieve a resistance of $5 \Omega$ with an electrode buried depth of $2 \mathrm{~m}$. Although at lower soil resistivity values the reduction in electrode length is smaller, about $2 \mathrm{~m}$ for $500 \Omega \mathrm{m}$ compared to a burial a depth of $0.5 \mathrm{~m}$, a reduction of approximately $13 \mathrm{~m}$ is observed at higher soil resistivity value of $3000 \Omega \mathrm{m}$, as depicted in Fig 6.11.

With the frequency independent soil resistivity, the effective length of the electrode is constant at all frequencies. However, the actual resistivity changes with frequency. It can be observed from results of this research that the earth resistivity of $3000 \Omega \mathrm{m}$ at $100 \mathrm{~Hz}$ drops to $329 \Omega \mathrm{m}$ at $4 \mathrm{MHz}$. Hence, the effective length of an electrode required at a higher frequency is shorter than that required at lower frequencies.

According to IEC 61400-24, electrode lengths greater than $80 \mathrm{~m}$ have very little influence on the WTG impedance value at high frequencies. On the contrary, the standard recommends to achieve resistance less than $10 \Omega$ at a frequency different from the lower order harmonics of the power frequency. Hence, in order to achieve a low resistance at low frequencies for higher soil resistivity WTG sites, longer length of electrodes can be applied. This is evident from Table 6.1, an electrode length of $187 \mathrm{~m}$ is recommended to achieve a resistance $5 \Omega$ for $3000 \Omega$ m earth resistivity. 


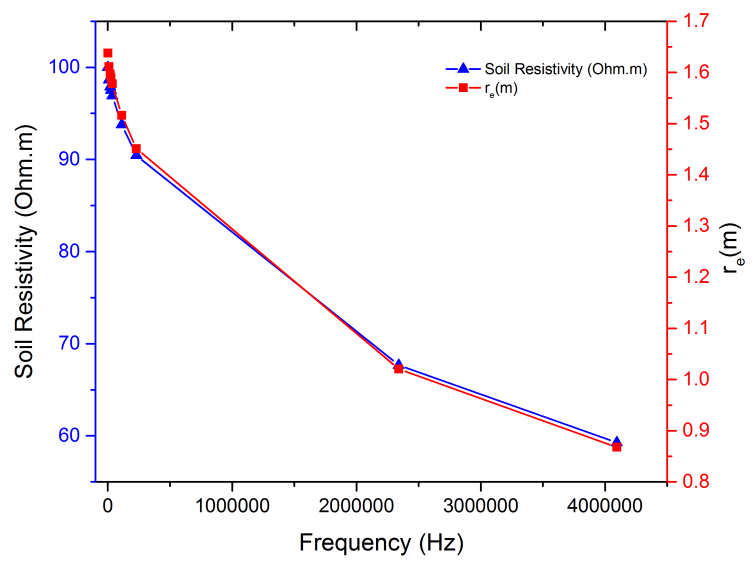

(a) $100 \Omega \mathrm{m}$

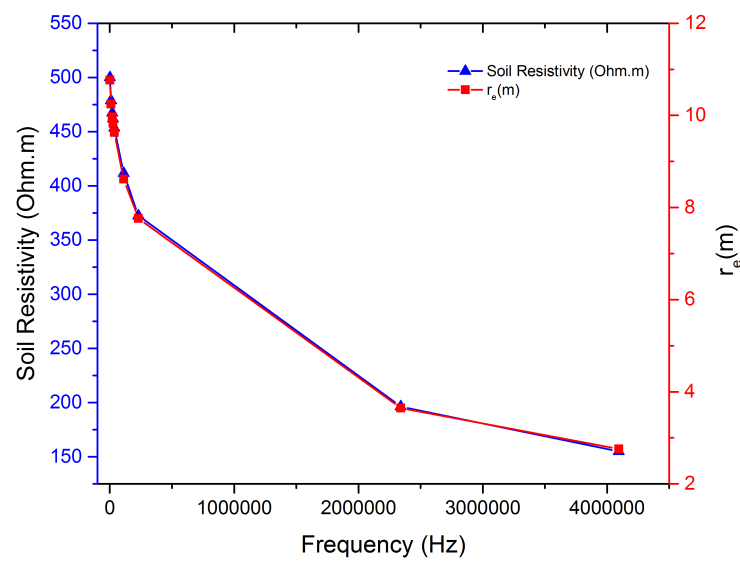

(b) $500 \Omega \mathrm{m}$

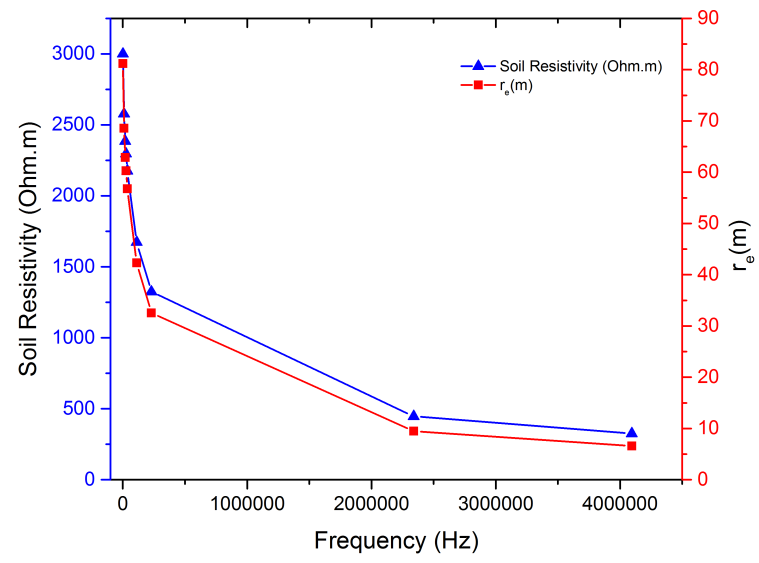

(c) $3000 \Omega \mathrm{m}$

Figure 6.9: WTG earth electrode length for $10 \Omega$ resistance for $2 \mathrm{~m}$ buried depth with the proposed method. 


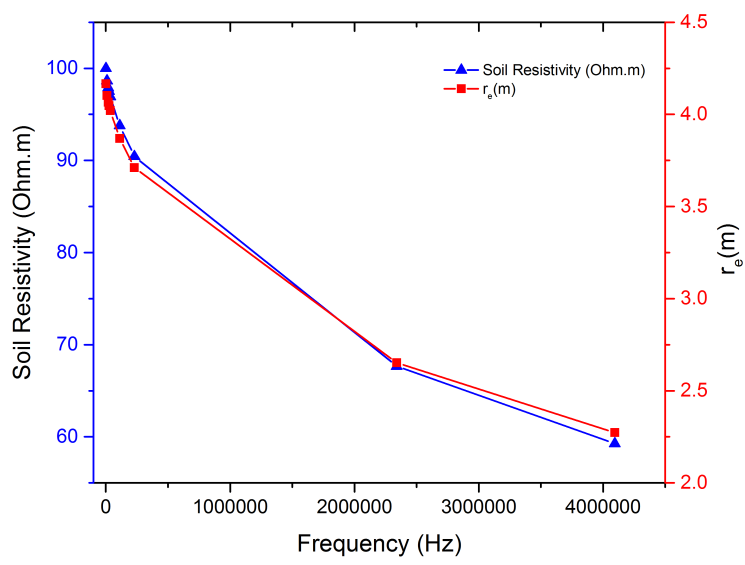

(a) $100 \Omega \mathrm{m}$

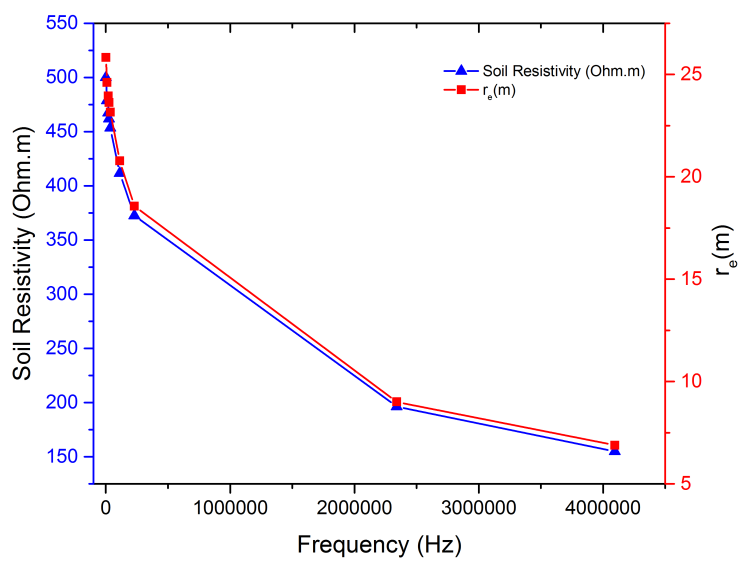

(b) $500 \Omega \mathrm{m}$

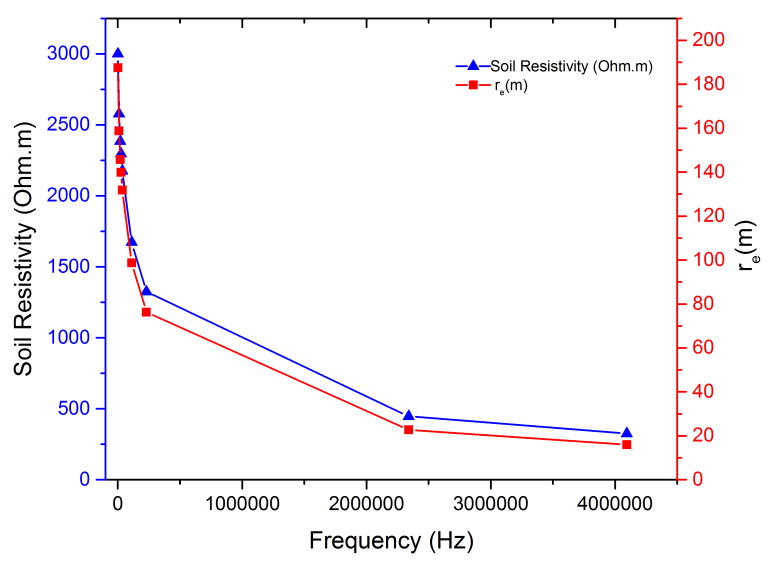

(c) $3000 \Omega \mathrm{m}$

Figure 6.10: WTG earth electrode length for $5 \Omega$ resistance for $0.5 \mathrm{~m}$ buried depth with the proposed method. 


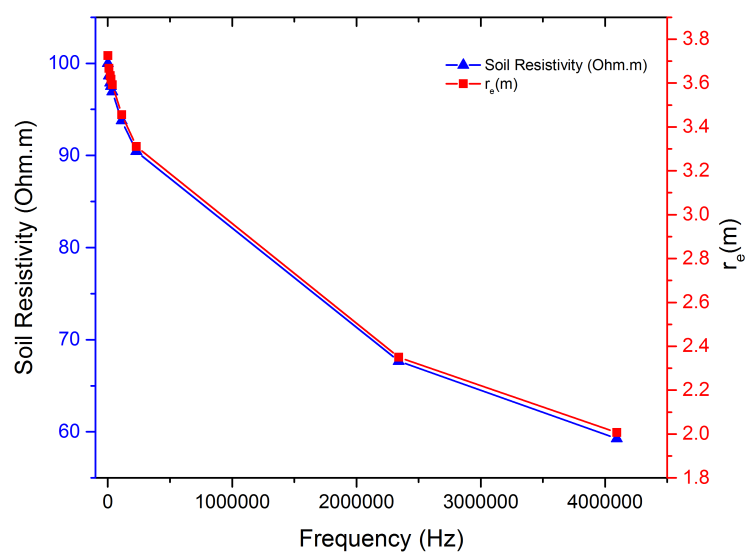

(a) $100 \Omega \mathrm{m}$

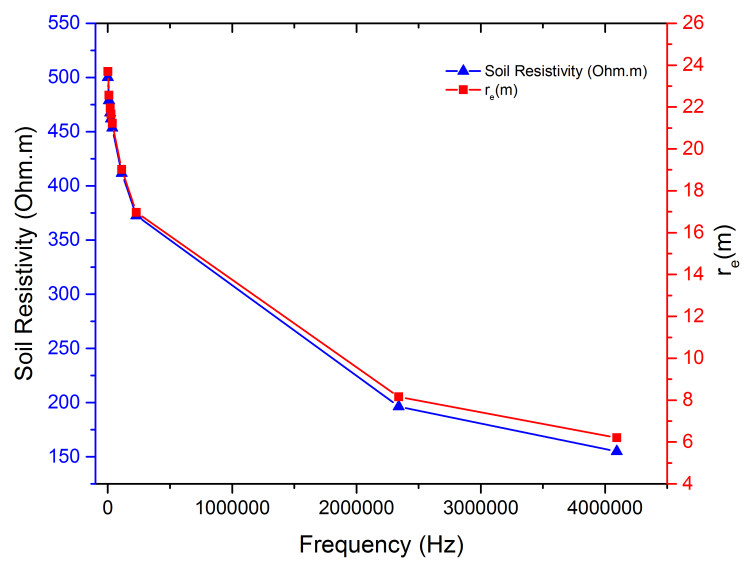

(b) $500 \Omega \mathrm{m}$

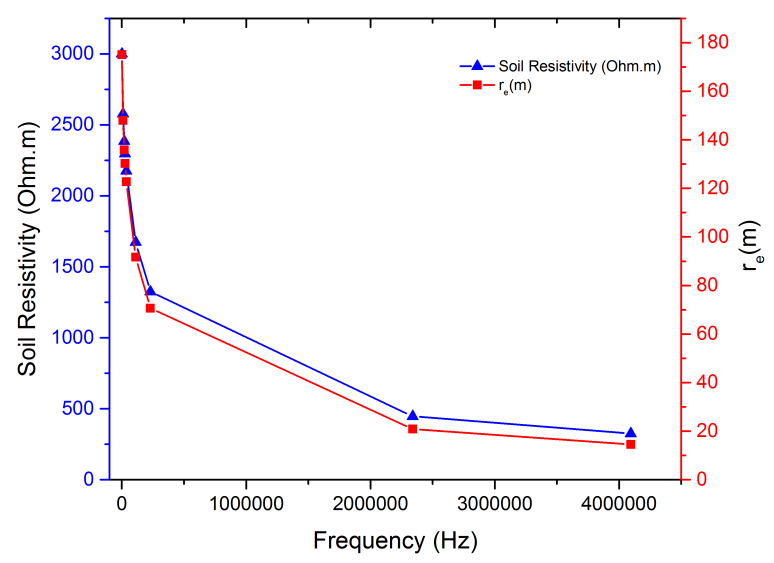

(c) $3000 \Omega \mathrm{m}$

Figure 6.11: WTG earth electrode length for $5 \Omega$ resistance for $2 \mathrm{~m}$ buried depth with the proposed method. 


\subsubsection{Case Study: Three proposed wind sites in Australia}

The proposed method is implemented for three WTG sites, where a soil resistivity measurement campaign was conducted by a collaborator [34] in two traverse directions. The measured soil resistivity values are stratified as horizontal two-layer and uniform soil resistivity models. In this work, WTG-1-T1 represents the traverse-1 soil resistivity values for the wind turbine site-1. WTG-1-T2 is traverse-2 soil resistivity measurements for wind turbine site-1. WTG-1-T1\&T2 represents the soil structure model for the combined soil resistivity values of traverse one and two for wind turbine site-1.

In addition, the proposed method makes use of an equivalent soil resistivity for calculating the electrode length. The equivalent soil resistivity values are calculated at two different lightning discharge current frequencies of $5 \mathrm{kHz}$ and $1 \mathrm{MHz}$. These frequencies are essential for first negative stroke lightning discharge current, as $1 \mathrm{MHz}$ represents the dominant frequency at peak of the lighting discharge current waveform, and $5 \mathrm{kHz}$ represents the half value at the wave tail.

Finally, the wind turbine earthing impedance is calculated by designing the electrode lengths with four scenarios for each traverse of the WTGs, as described in Table 6.2. In the first case, the soil is modelled with uniform resistivity, and the electrode length is calculated according to the uniform soil resistivity value. The second scenario is based on the twolayer soil model, and the electrode length is designed based on the soil resistivity of the top layer of the two-layer model. In the third scenario, the electrode length is calculated based on the bottom layer resistivity of the two-layer soil model. In the first three cases, the electrode length is calculated based on the traditional method provided in IEC 61400-24. The proposed method is implemented in the final case to calculate the electrode length.

Table 6.3 depicts the soil structures for each of the WTG sites measured in two traverses, T1 and T2, and the combination of T1 \& T2. In this work, 
Table 6.2: Simulation scenarios

\begin{tabular}{|l|c|}
\hline Scenario-1 & Uniform soil resistivity model \\
\hline Scenario-2 & $\begin{array}{c}\text { Soil resistivity selected from the top layer } \\
\text { of the horizontal two-layer soil model }\end{array}$ \\
\hline Scenario-3 & $\begin{array}{c}\text { Soil resistivity selected from the bottom layer } \\
\text { of the horizontal two-layer soil model }\end{array}$ \\
\hline Scenario-4 & Proposed method \\
\hline
\end{tabular}

measured soil resistivity values in two traverses are modelled separately, as $\mathrm{T} 1, \mathrm{~T} 2$, and the combination of $\mathrm{T} 1 \& \mathrm{~T} 2$, to represent the soil structure more realistically.

It can be observed from Table 6.3 that the earth resistivity varies from $880 \Omega \mathrm{m}$ to $1006 \Omega \mathrm{m}$ for uniform soil structure of WTG-1 in different traverses. The variation in top layer soil resistivity of WTG-1 for two-layer soil structure ranges from $758 \Omega$.m to $2593 \Omega \mathrm{m}$, and the bottom layer resistivity varies from $759 \Omega . \mathrm{m}$ to $1175 \Omega \mathrm{m}$. In addition, the top layer thickness differs significantly from $0.37 \mathrm{~m}$ to $28.89 \mathrm{~m}$. In such a situation, with the electrode depth at $0.5 \mathrm{~m}$, the earth electrodes are always in the bottom layer. However, the wind turbine foundation rebar and other metal components extend from the surface of the earth through the top layer to the bottom layer. The diversified earth resistivity and layer thickness makes the calculation of electrode length difficult. Hence, this research utilises the equivalent soil resistivity for calculating the electrode length.

The next step in the design of the WTG earthing system is calculating the electrode lengths which depend on the soil resistivity value, electrode dimensions and the electrode burial depth. For the case of a horizontal two-layer structure, it is difficult to choose the soil resistivity of the two layers in the calculation, as the variation in soil resistivity and the top layer depths are diverse. Thus, the method described in IEC-61400 24 is not practical for calculating earth electrode length for a two-layer soil structure. 


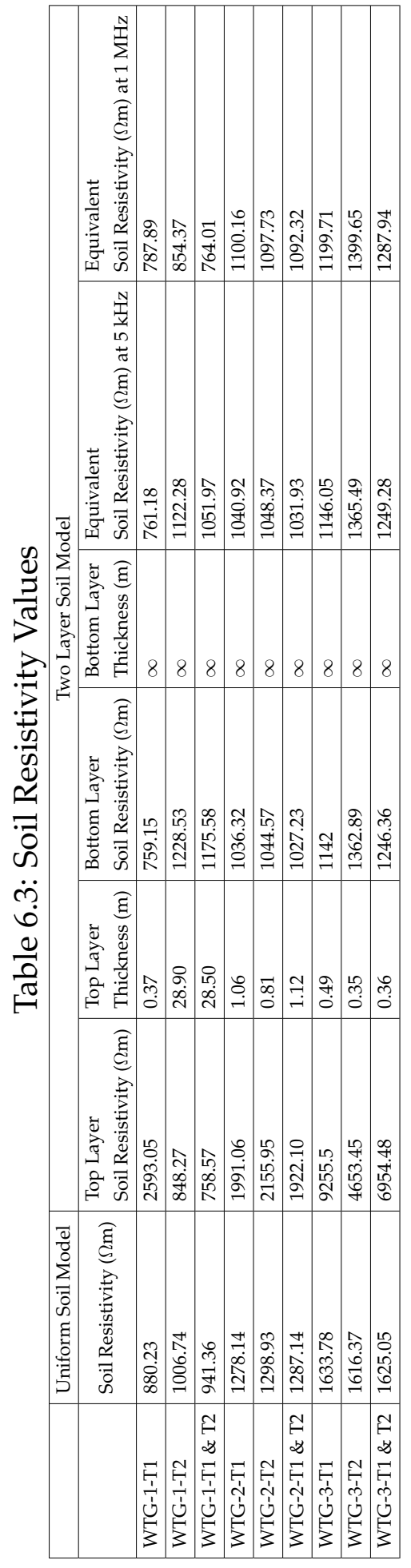


Table 6.4 presents the calculated electrode lengths for the four scenarios considered in this research. The minimum length of the electrode required for WTG-1-T1 assuming a uniform soil resistivity model is about $16.41 \mathrm{~m}$. The electrode length required for the same WTG when the length of the electrode is calculated based on the top layer of the horizontal two-layer soil model is about $67.79 \mathrm{~m}$. The electrode length calculated based on the bottom layer of the horizontal two-layer soil model is about $12.77 \mathrm{~m}$. These variations are for the WTG in the same traverse. However, the electrode lengths calculated with the soil resistivity values of traverse- 2 are $20.2 \mathrm{~m}$, $15.45 \mathrm{~m}$, and $26.86 \mathrm{~m}$, respectively for three scenarios. Moreover, the electrode lengths for soil resistivity values of combined traverse- 1 and 2 are $18.24 \mathrm{~m}, 12.76 \mathrm{~m}$, and $25.27 \mathrm{~m}$. The fluctuation in electrode length is large, ranging from $16 \mathrm{~m}$ to $68 \mathrm{~m}$ for the same WTG.

The conventional method considers the mean radius of the ring electrode and the length of the electrode as the same quantity. However, there is a difference in the calculated earthing impedance with a ring earth electrode and horizontal or vertical electrodes. Hence, the conversion of the mean radius of an electrode to equivalent horizontal length is essential for a WTG earthing system.

Table 6.4 also shows the electrode lengths calculated with the proposed method at $5 \mathrm{kHz}$ and $1 \mathrm{MHz}$ frequencies. The mean radius of the ring electrode required at $5 \mathrm{kHz}$ for WTG-1-T1 is $18.2 \mathrm{~m}$. However, the actual radius of the ring electrode is $10 \mathrm{~m}$, and hence the horizontal electrode length of $51.58 \mathrm{~m}$, calculated using equation 6.7 can be used to fulfil the electrode length.

It can be observed that the recommended minimum electrode length for WTG-1-T1 varies from $12 \mathrm{~m}$ to $68 \mathrm{~m}$ for WTG-1-T1 depending on how soil resistivity is modelled, whereas with the proposed method the length is $18 \mathrm{~m}$. The variation is greater for a high soil resistivity site such as WTG$3-\mathrm{T} 1$, which ranges from $24 \mathrm{~m}$ to $80 \mathrm{~m}$. The proposed method gives an electrode length of $29 \mathrm{~m}$ for WTG-3-T1. 


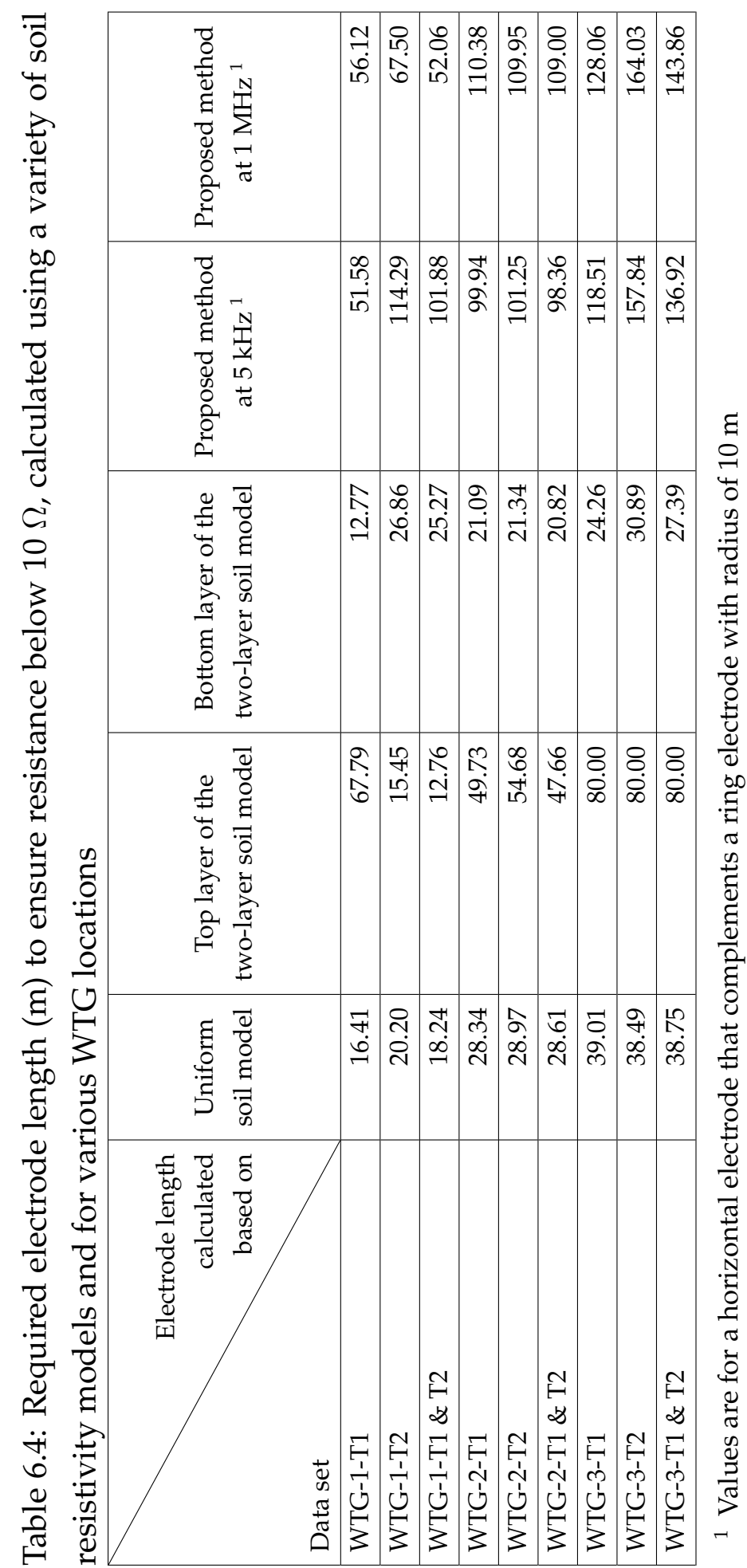


Table 6.5: WTG Earthing Impedance

\begin{tabular}{|c|c|c|c|c|c|}
\hline WTG & \multicolumn{5}{|c|}{ WTG Earthing Impedance $(\Omega)$} \\
\hline & $\begin{array}{c}\text { Uniform } \\
\text { soil } \\
(5 \mathrm{kHz})\end{array}$ & $\begin{array}{c}\text { Top layer } \\
\text { of two layer } \\
(5 \mathrm{kHz})\end{array}$ & $\begin{array}{c}\text { Bottom layer } \\
\text { of two layer } \\
(5 \mathrm{kHz})\end{array}$ & $\begin{array}{c}\text { Proposed } \\
\text { method } \\
(5 \mathrm{kHz})\end{array}$ & $\begin{array}{c}\text { Proposed } \\
\text { method } \\
(1 \mathrm{MHz})\end{array}$ \\
\hline WTG-1-T1 & 17.07 & 9.13 & 15.26 & 9.51 & 22.02 \\
\hline WTG-1-T2 & 18.97 & 17.42 & 15.77 & 9.09 & 22.04 \\
\hline WTG-1-T1 \& T2 & 18.04 & 15.92 & 14.54 & 9.13 & 22.00 \\
\hline WTG-2-T1 & 22.25 & 15.95 & 20.42 & 9.14 & 21.87 \\
\hline WTG-2-T2 & 22.37 & 14.29 & 19.97 & 9.15 & 21.88 \\
\hline WTG-2-T1 \& T2 & 22.28 & 16.23 & 20.28 & 9.17 & 21.88 \\
\hline WTG-3-T1 & 25.32 & 12.63 & 21.29 & 9.06 & 21.73 \\
\hline WTG-3-T2 & 25.18 & 14.87 & 23.35 & 8.97 & 21.36 \\
\hline WTG-3-T1 \& T2 & 25.19 & 13.65 & 22.34 & 9.01 & 21.57 \\
\hline
\end{tabular}

The impedance of the WTG earthing system for all the scenarios is depicted in Table 6.5. The grounding impedance of WTG-1-T1 with electrode length based on uniform soil resistivity is $17.07 \Omega, 9.13 \Omega$ with the electrode length based on top layer resistivity of the two-layer model and $15.26 \Omega$ with electrode length based on the bottom layer soil resistivity of the twolayer structure, at a frequency of $5 \mathrm{kHz}$. However, the WTG grounding impedance with the proposed method are $9.51 \Omega$ and $22.02 \Omega$ at $5 \mathrm{kHz}$ and $1 \mathrm{MHz}$, respectively.

The ground impedance values of WTG-2-T1 are $22.25 \Omega, 15.95 \Omega$, and $20.42 \Omega$ based on the electrode lengths calculated with uniform and, top and bottom layer resistivity of the two-layer soil structure, at $5 \mathrm{kHz}$. The impedance value reduces to $9.14 \Omega$ with the proposed method. It can be observed from the results that WTG grounding impedance of less than $10 \Omega$ at low frequencies is achieved for all the WTG sites, whereas with the traditional method the impedance values remain more than $10 \Omega$ except for one of the cases. 


\subsubsection{Potential Distribution}

This section explains the potential distribution of the WTG grounding system at a frequency of $5 \mathrm{kHz}$ for an earthing design based on all the scenarios for WTG-3-T1. It is observed that the WTG potential profile exhibits an equipotential surface for the low frequencies with a variation in the peak potential rise value. Figure 6.12 depicts the potential profile for the case of an earthing design based on a uniform soil model.

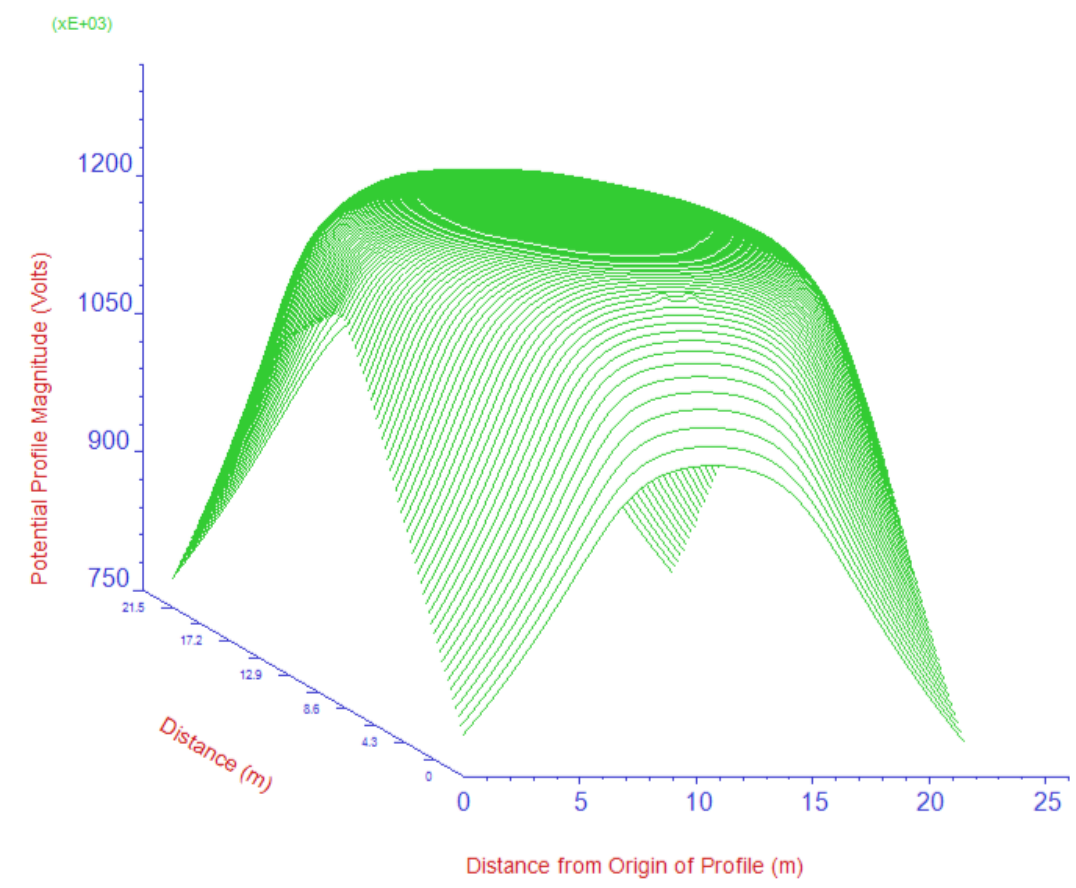

Figure 6.12: Potential distribution for the electrode design based on uniform soil resistivity.

The variation in potential distribution ranges from $782.03 \mathrm{kV}$ to $1266.39 \mathrm{kV}$. The potential rise of the grounding grid for an earthing system design based on the top and bottom layer resistivities for a two-layer soil are shown in Figures 6.13 and 6.14. It can be observed that the potential value ranges from $405.19 \mathrm{kV}$ to $632.08 \mathrm{kV}$ and $617.41 \mathrm{kV}$ to $1064.98 \mathrm{kV}$, 


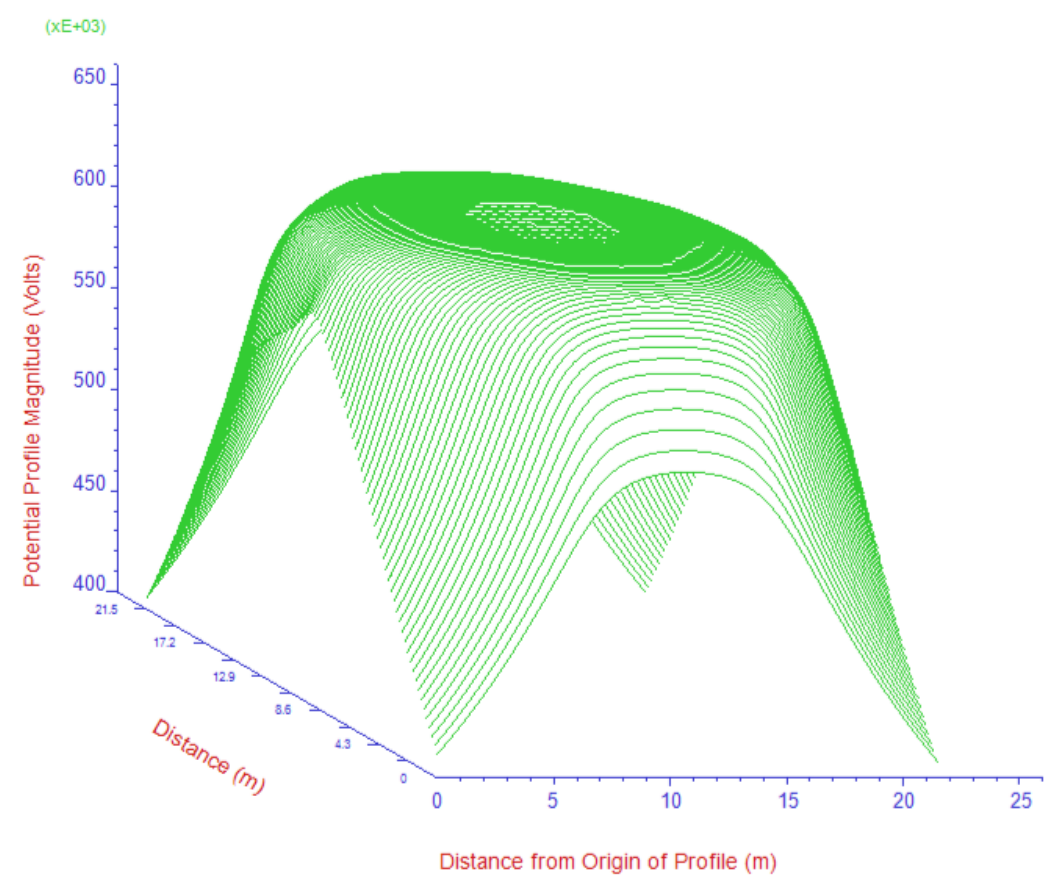

Figure 6.13: Potential distribution for the electrode design based on top layer soil resistivity of the two-layer soil model. 


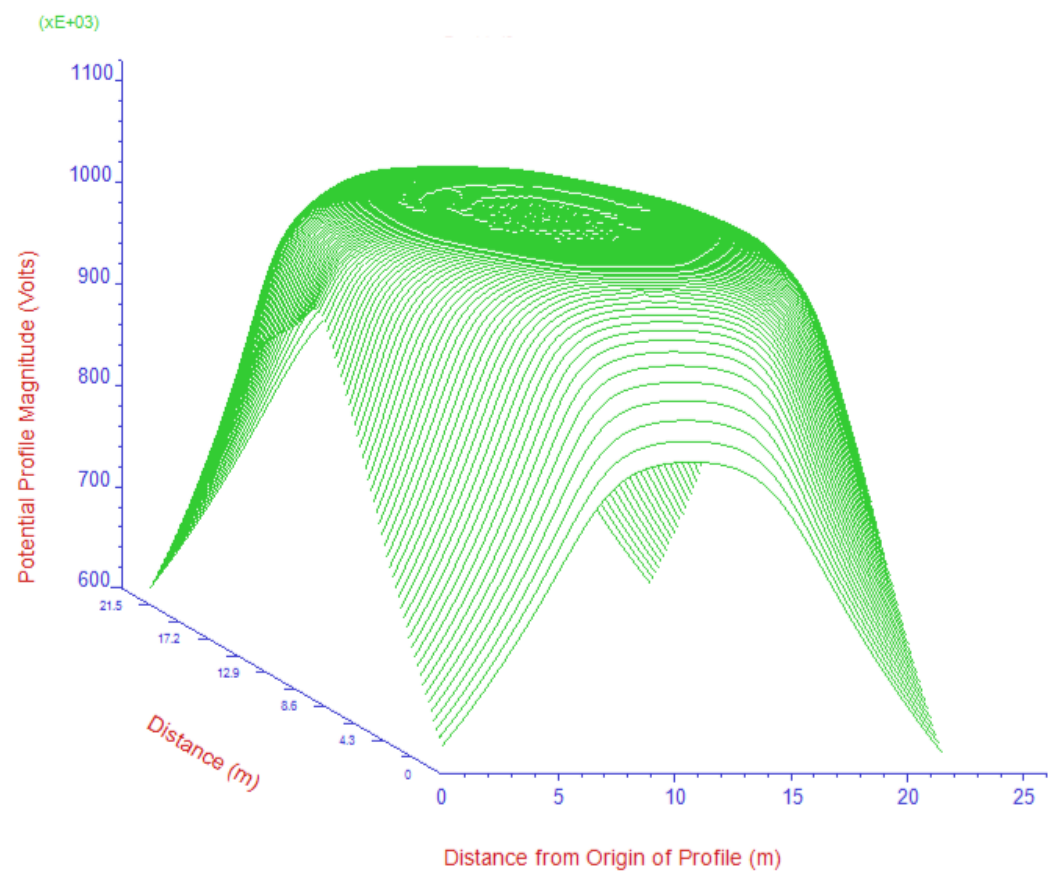

Figure 6.14: Potential distribution for the electrode design based on bottom layer soil resistivity of the two-layer soil model. 


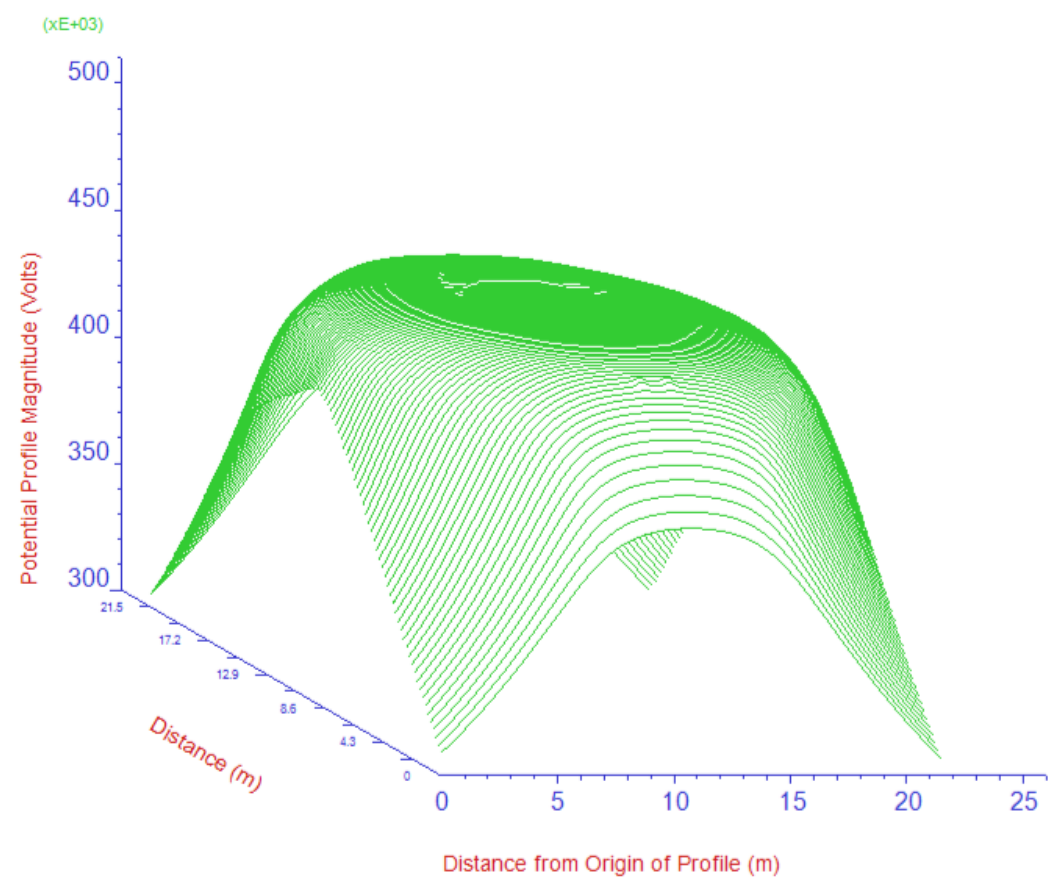

Figure 6.15: Potential distribution for the electrode design based on the proposed method. 
for the electrodes design based on the top and bottom layer resistivites, respectively. However, the proposed method limits the potential rise to a minimum value amongst all the cases with potential rise ranging from $304.7 \mathrm{kV}$ to $453.32 \mathrm{kv}$, as depicted in Figure 6.15.

\subsection{Chapter summary}

This research proposes a novel method to calculate the minimum length of an earth electrode for uniform and two-layer based soil models. The grounding impedance achieved when electrode lengths are calculated using the proposed method is compared to grounding impedance values calculated using the conventional method. This comparison shows that the proposed method is an improvement on the current convention. In particular, the proposed method gives a grounding impedance value of less than $10 \Omega$ at low frequencies for all soil resistivity values. This results in a reduction in the potential rise of up to $64 \%$ compared to the peak potential value in the conventional method. Finally, the smallest difference between the minimum and maximum values of the potential rise is also indicated with the proposed method.

The benefits offered by the proposed method mean that it can be employed to calculate electrode lengths for the required resistance values based on soil resistivity, electrode dimensions, and burial depth. Such a design may serve as a starting point for an engineer wishing to design a WTG earthing system. 


\section{Chapter 7}

\section{Conclusions and Future Work}

\subsection{Conclusions}

To achieve this challenging task, five objectives were defined for this research. The research in this thesis presents an analysis and design of improved grounding system for lightning discharge currents. It start with the analysis of soil resistivity modelling for a wind turbine grounding system considering the relevant parameters. Research into developing a framework to assess effectiveness of WTG lightning protection system was performed. This analysis also looked into the effect of various lightning protection level and lightning discharge current parameters on WTG grounding system. In addition, a study on various electrode configurations to achieve the research objectives was performed. Finally, a method was proposed to improve the design of earth electrodes of a WTG grounding system.

This thesis incorporates the following major contributions:

1. This thesis discusses a detailed analysis of wind turbine earthing impedance considering several factors. Firstly, the analysis of soil modelling of the measured soil resistivity values is performed. Secondly, the effect of frequency dependent soil parameters on 
the WTG earthing impedance is quantified. Next, the effects of rebar and horizontal electrodes on the WTG earthing impedance are evaluated. Also, the effect of earth electrodes in various soil layers of a horizontal two-layer soil model is performed. Finally, the distribution of potential around the WTG foundation at various frequencies for different designs of grounding system when lightning strikes on a WTG is analysed. The findings of this research have been peer-reviewed and published in:

2. This research developed a framework for assessing the effectiveness of WTG lightning protection system. The installation of WTGs at low probability of lightning incidence and the design of effective grounding systems are the parameters controllable by the wind farm designers. This work explains that the effectiveness of the grounding system can be improved by proper design of earth electrodes, soil stratification, and low resistivity soil sites. In addition, the impact on the grounding system for different parameters of lightning protection level and lightning current is analysed and concluded that designing a LPL-1 is effective and consideration of the sub-sequent lightning discharge current parameters is important because of the higher frequencies compared to first strokes. The findings of this research have been peer-reviewed and published in: Publication is submitted to IEEE Transactions on Industry Applications and is accepted for publication:

3. This research proposes a novel method to calculate the minimum length of an earth electrode for a two-layer based soil model. The grounding impedance achieved when electrode lengths are calculated using the proposed method is compared to grounding impedance values calculated using the conventional method. This 
comparison shows that the proposed method is an improvement on the current convention. In particular, the proposed method gives a grounding impedance value of less than $10 \Omega$ at low frequencies for all soil resistivity values. This results in a reduction in the potential rise of up to $64 \%$ compared to the peak potential value in the conventional method. Moreover, the benefits offered by our proposed method mean that it can be employed to calculate electrode lengths for the required resistance values based on soil resistivity, electrode dimensions, and buried depth. Such a design may serve as a starting point for an engineer wishing to design a WTG earthing system. The findings of this research have been peer-reviewed and published in:

4. The analysis of designing a grounding system to various lightning discharge current and lightning protection level parameters to select the appropriate design parameters is presented. This analysis guides the wind farm designers in selecting correct parameters for the grounding system design. The analysis of various lightning current waveforms identifies the frequency components and their effect on the WTG earthing system. It is observed that the high-frequency components are more dominant in determining the potential rise and impedance, which are more dominant in subsequent lightning strokes. The findings of this research have been peer-reviewed and published in:

5. Finally, through modelling and analysis of various earth electrodes for a WTG earthing system based on uniform and horizontal soil models, this thesis proposes an optimum electrode configuration to achieve a low impedance for the wind turbine earthing impedance. WTG earthing impedances for various lightning discharge currents and associated high frequencies are compared, and recommenda- 
tions are made for an improved earthing system. The findings of this research have been peer-reviewed and published in:

These conclusions will help inform the design of future WTG grounding systems to facilitate the continued worldwide adoption of WTGs as a source of clean and sustainable power.

\subsection{Future Work}

During the course of this research, various opportunities were identified that could be used to further enhance the WTG lightning protection system. These are mentioned below:

- One of the next steps in this research is to expand the analysis developed here to consider an LPS for an entire wind farm. This poses a challenge as individual wind turbines in a wind farm have various soil resistivity and lightning incidence probability. The interconnected wind turbine can have both positive and negative effects. The positive side is, the interconnected wind turbines through a conductor can reduce the low-frequency resistance. However, the interconnection may transfer over-voltages in the wind farm even when a single WTG is struck by lightning.

- This research proposed a method to calculate the minimum length of the earth electrodes. However, a generalised approach for all the soil models can be considered as for future work. Future iterations should also incorporate step and touch voltage requirements alongside maximum allowable resistance values into the electrode length calculations, further simplifying the overall design process.

- The analysis and design of WTG grounding system in this thesis considered the complete rebar of the WTG foundation. However, the 
consideration of concrete in the foundation poses new challenges as the resistivity may vary for different scenarios. Hence, the consideration of complete concrete can be considered.

- This research considered measured soil resistivity for the design and analysis of WTG grounding system. The measurement of soil resistivity after the installation of WTGs can help the designers to apply a correction factor.

- The permissible levels of step and touch voltages are available at power frequency and hence the WTG grounding designs can be verified for limits. There is a need for a standard to verify the step and touch voltages at higher frequencies.

- Finally, practical testing of the WTG earthing system, according to this thesis, can be performed for a better understanding of the WTG earthing system for lightning discharge currents.

In summary, this thesis explored the state of the art in WTG LPS design and identified several improvements that could be implemented to make WTGs safer and more reliable. Adoption of these recommendations by industry groups, manufacturers and operators will facilitate the continued growth of wind energy as an efficient, reliable, and environmentally friendly means of power generation. 


\section{Bibliography}

[1] M. H. Ali, Wind energy systems: solutions for power quality and stabilization. CRC Press, 2016.

[2] M. Ishii, M. Saito, D. Natsuno, and A. Sugita, "Lightning incidence on wind turbines in winter," in 2014 International Conference on Lightning Protection (ICLP), Oct 2014, pp. 1734-1738.

[3] H. Heckler, J. Wosgien, and M. Wetter, “New lightning current arrester design for onshore and offshore wind turbines," in 2011 International Symposium on Lightning Protection, Oct 2011, pp. 241-246.

[4] M. Long, M. Becerra, and R. Thottappillil, "On the lightning incidence to wind farms," in 2016 33rd International Conference on Lightning Protection (ICLP), Sep. 2016, pp. 1-5.

[5] J. Birkl, G. Diendorfer, S. Thern, J. Kolb, E. Shulzhenko, and M. Rock, "Initial investigation of influence of wind farms to lightning events," in 2016 33rd International Conference on Lightning Protection (ICLP), Sep. 2016, pp. 1-7.

[6] Z. Guo, Q. Li, W. Yu, W. Arif, Y. Ma, and W. H. Siew, “Experimental study on lightning attachment manner to rotation wind turbine blade," in 2018 34th International Conference on Lightning Protection (ICLP), Sep. 2018, pp. 1-5. 
[7] N. Tsutsui, K. Yamamoto, Y. Kasai, N. Honjo, K. Takahashi, T. Goda, H. Takagi, and Y. Naito, "Study of wind turbine generation system shutdown using atmospheric electric field potential," in $201633 \mathrm{rd}$ International Conference on Lightning Protection (ICLP), Sep. 2016, pp. $1-4$.

[8] J. Sawin et al., "Renewable energy policy network for the 21st century renewables 2017 global status report," REN21 Secretariat: Paris, France, pp. 1-302, 2017.

[9] B. Sliz-Szkliniarz, J. Eberbach, B. Hoffmann, and M. Fortin, "Assessing the cost of onshore wind development scenarios: Modelling of spatial and temporal distribution of wind power for the case of poland," Renewable and Sustainable Energy Reviews, vol. 109, pp. 514 - 531, 2019. [Online]. Available: http: / / www.sciencedirect.com/science/article/pii/S1364032119302515

[10] S. Yokoyama, "Lightning protection of wind turbine blades," Electric Power Systems Research, vol. 94, pp. 3 - 9, 2013, lightning Protection of Advanced Energy Systems. [Online]. Available: http: / / www.sciencedirect.com/science/article/pii/S0378779612002179

[11] Yang Tingfang and Yang Xin, "Lightning protection of wind turbines," in 2010 The 2nd International Conference on Industrial Mechatronics and Automation, vol. 1, May 2010, pp. 60-63.

[12] R. Rodrigues, V. Mendes, and J. Catalão, "Protection of interconnected wind turbines against lightning effects: Overvoltages and electromagnetic transients study," Renewable Energy, vol. 46, pp. 232 - 240, 2012. [Online]. Available: http: / / www.sciencedirect.com/science/article/pii/S0960148112002030

[13] H. T. Zavareh, "Wind turbines protection against the lightning struck using a combined method," in 2012 Second Iranian Conference 
on Renewable Energy and Distributed Generation, March 2012, pp. 5154.

[14] X. Zhang, Y. Zhang, and X. Xiao, "An improved approach for modeling lightning transients of wind turbines," International Journal of Electrical Power E Energy Systems, vol. 101, pp. 429-438, 2018.

[15] M. Minowa, K. Ito, S. I. Sumi, and K. Horii, "A study of lightning protection for wind turbine blade by using creeping discharge characteristics," in 2012 International Conference on Lightning Protection (ICLP), Sep. 2012, pp. 1-4.

[16] R. Shariatinasab, B. Kermani, and J. Gholinezhad, "Transient modeling of the wind farms in order to analysis the lightning related overvoltages," Renewable Energy, vol. 132, pp. 1151 - 1166, 2019. [Online]. Available: http://www.sciencedirect.com/science/ article/pii/S0960148118310346

[17] International Electrotechnical Commission, "IEC 61400-24 Wind Turbines-part 24: Lightning Protection," 2010.

[18] A. Sunjerga, Q. Li, D. Poljak, M. Rubinstein, and F. Rachidi, "Isolated vs. interconnected wind turbine grounding systems: Effect on the harmonic grounding impedance, ground potential rise and step voltage," Electric Power Systems Research, vol. 173, pp. 230 - 239, 2019. [Online]. Available: http://www.sciencedirect.com/ science/article/pii/S0378779619301373

[19] F. Rachidi, M. Rubinstein, J. Montanya, J. Bermudez, R. Rodriguez Sola, G. Sola, and N. Korovkin, "A review of current issues in lightning protection of new-generation wind-turbine blades," IEEE Transactions on Industrial Electronics, vol. 55, no. 6, pp. 2489-2496, June 2008. 
[20] J. Niihara, A. Ametani, and K. Yamamoto, "Transient grounding characteristics of wind turbine with counterpoise," in 2012 AsiaPacific Symposium on Electromagnetic Compatibility, May 2012, pp. 869-872.

[21] D. S. Gazzana, A. Smorgonskiy, N. Mora, A. Šunjerga, M. Rubinstein, and F. Rachidi, "An experimental field study of the grounding system response of tall wind turbines to impulse surges," Electric Power Systems Research, vol. 160, pp. 219 - 225, 2018. [Online]. Available: http: / / www.sciencedirect.com/science/article/pii/S0378779618300634

[22] O. Ukar and I. Zamora, "Wind farm grounding system design for transient currents," Renewable Energy, vol. 36, no. 7, pp. 2004 - 2010, 2011. [Online]. Available: http://www.sciencedirect.com/science/ article/pii/S096014811100005X

[23] P. Sarajcev and R. Goic, "Assessment of lightning current parameters suitable for wind turbine overvoltage protection analysis," Wind Energy, vol. 15, no. 4, pp. 627-644. [Online]. Available: https: / / onlinelibrary.wiley.com/doi/abs/10.1002/we.496

[24] N. Malcolm and R. K. Aggarwal, "Estimation of the failure rate of wind turbine electrical systems exposed to lightning strikes," in 2015 IEEE Power Energy Society General Meeting, July 2015, pp. 1-5.

[25] N. Malcolm and R. Aggarwal, "Analysis of transient overvoltage phenomena due to direct lightning strikes on wind turbine blade," in 2014 IEEE PES General Meeting I Conference Exposition, July 2014, pp. $1-5$.

[26] R. Rodrigues, V. Mendes, and J. Catalão, "Protection of wind energy systems against the indirect effects of lightning," Renewable Energy, 
vol. 36, no. 11, pp. 2888 - 2896, 2011. [Online]. Available: http: //www.sciencedirect.com/science/article/pii/S0960148111001832

[27] P. Sarajčev, I. Sarajčev, and R. Goić, "Transient emf induced in $\mathrm{lv}$ cables due to wind turbine direct lightning strike," Electric Power Systems Research, vol. 80, no. 4, pp. 489 - 494, 2010. [Online]. Available: http://www.sciencedirect.com/science/ article/pii/S0378779609002739

[28] V. Peesapati, "Lightning protection of wind turbines," Ph.D. dissertation, University of Manchester, 2010.

[29] B. M. Radičević, M. S. Savić, S. F. Madsen, and I. Badea, “Impact of wind turbine blade rotation on the lightning strike incidence a theoretical and experimental study using a reduced-size model," Energy, vol. 45, no. 1, pp. $644-654$, 2012, the 24th International Conference on Efficiency, Cost, Optimization, Simulation and Environmental Impact of Energy, ECOS 2011. [Online]. Available: http: //www.sciencedirect.com/science/article/pii/S0360544212005622

[30] L. Grcev and M. Popov, "On high-frequency circuit equivalents of a vertical ground rod," IEEE Transactions on Power Delivery, vol. 20, no. 2, pp. 1598-1603, April 2005.

[31] J. Takami and S. Okabe, "Observational results of lightning current on transmission towers," IEEE Transactions on Power Delivery, vol. 22, no. 1, pp. 547-556, Jan 2007.

[32] S. Gholami Farkoush, A. Wadood, T. Khurshaid, C. Kim, M. Irfan, and S. Rhee, "Reducing the effect of lightning on step and touch voltages in a grounding grid using a nature-inspired genetic algorithm with atp-emtp," IEEE Access, vol. 7, pp. 81 903-81910, 2019.

[33] M. Mokhtari, Z. Abdul-Malek, and Z. Salam, "The effect of soil ionization on transient grounding electrode resistance in non- 
homogeneous soil conditions," International Transactions on Electrical Energy Systems, vol. 26, no. 7, pp. 1462-1475. [Online]. Available: https: / / onlinelibrary.wiley.com/doi/abs/10.1002/etep.2157

[34] R. D. Goud, T. Auditore, R. Rayudu, and C. P. Moore, "Frequency domain analysis of a wind turbine generator earthing system for lightning discharge currents," IEEE Access, vol. 7, pp. 60501-60 512, 2019.

[35] R. D. Goud, R. Rayudu, C. P. Moore, and T. Auditore, "An evaluation of potential rise in a wind turbine generator earthing system during a direct lightning strike," in 2018 International Conference and Utility Exhibition on Green Energy for Sustainable Development (ICUE), Oct 2018, pp. 1-7.

[36] R. D. Goud, T. Auditore, R. Rayudu, and C. P. Moore, "Analysis of earth electrodes of wind turbine generator grounding system under lightning discharge currents," in 2018 IEEE PES Asia-Pacific Power and Energy Engineering Conference (APPEEC), Oct 2018, pp. 502-507.

[37] R. D. Goud, T. Auditore, R. Rayudu, and C. P. Moore, "A proposed method for calculating earth electrode length for a wind turbine generator grounding system," in 2018 International Conference and Utility Exhibition on Green Energy for Sustainable Development (ICUE), Oct 2018, pp. 1-6.

[38] R. D. Goud, T. Auditore, R. Rayudu, C. P. Moore, and E. Sturov, "A new method for calculating earth electrode length for a wind turbine generator grounding system based on a two-layer soil structure," in 2018 International Conference on Power System Technology (POWERCON), Nov 2018, pp. 1270-1276.

[39] The inside of a wind turbine. http:/ / energy.gov/eere/wind/insidewind-turbine- 0 . 
[40] A. Yazdanbakhsh, L. C. Bank, K.-A. Rieder, Y. Tian, and C. Chen, "Concrete with discrete slender elements from mechanically recycled wind turbine blades," Resources, Conservation and Recycling, vol. 128, pp. 11 - 21, 2018. [Online]. Available: http://www. sciencedirect.com/science/article/pii/S0921344917302446

[41] Components of Wind Machines. [Online]. Available: https:/ / mragheb.com/NPRE\%20475\%20Wind\%20Power\% 20Systems /Components\%20of\%20Wind\%20Machines.pdf

[42] W. Qiao and D. Lu, "A survey on wind turbine condition monitoring and fault diagnosis-part i: Components and subsystems," IEEE Transactions on Industrial Electronics, vol. 62, no. 10, pp. 6536-6545, Oct 2015.

[43] M. A. Cooper and R. L. Holle, Meteorological Concepts Affecting Lightning Formation. Cham: Springer International Publishing, 2019, pp. 125-138. [Online]. Available: https://doi.org/10.1007/ 978-3-319-77563-0_13

[44] V. A. Rakov and M. A. Uman, Lightning: physics and effects. Cambridge University Press, 2003.

[45] B. M. Smirnov, Electric Processes in Atmosphere. Cham: Springer International Publishing, 2017, pp. 59-85. [Online]. Available: https:/ /doi.org/10.1007/978-3-319-30813-5_4

[46] A. Seminario-García, C. González-Morán, and P. Arboleya, "Stepped leader progression and speed evolution in a thunderstorm: Theoretical model," Energies, vol. 12, no. 13, 2019. [Online]. Available: https:/ /www.mdpi.com/1996-1073/12/13/2507

[47] D. J. Malan, Physics of lightning. English Universities Press, 1964. 
[48] D. I. B. Djalel, M. Mourad, and S. Ghoudelbourk, "Protection of wind turbine against the lightning damage," in IREC2015 The Sixth International Renewable Energy Congress, March 2015, pp. 1-6.

[49] D. Djalel, G. Abdallah, and L. Hocine, "Study of the lightning impact on the wind-turbine," Energy Research Journal, vol. 5, no. 1, p. 17, 2014.

[50] N. Pineda, J. Montanyà, A. Salvador, O. A. van der Velde, and J. A. López, "Thunderstorm characteristics favouring downward and upward lightning to wind turbines," Atmospheric Research, vol. 214, pp. 46 - 63, 2018. [Online]. Available: http://www.sciencedirect. com/science/article/pii/S0169809518300735

[51] M. Long, M. Becerra, and R. Thottappillil, "On the attachment of dart lightning leaders to wind turbines," Electric Power Systems Research, vol. 151, pp. 432 - 439, 2017. [Online]. Available: http: //www.sciencedirect.com/science/article/pii/S0378779617302602

[52] Q. Zhou, C. Liu, X. Bian, K. L. Lo, and D. Li, "Numerical analysis of lightning attachment to wind turbine blade," Renewable Energy, vol. 116, pp. 584 - 593, 2018. [Online]. Available: http: //www.sciencedirect.com/science/article/pii/S0960148117309540

[53] A. Borghetti, C. A. Nucci, and M. Paolone, "Estimation of the statistical distributions of lightning current parameters at ground level from the data recorded by instrumented towers," IEEE Transactions on Power Delivery, vol. 19, no. 3, pp. 1400-1409, July 2004.

[54] A. J. Eriksson, "The incidence of lightning strikes to power lines," IEEE Power Engineering Review, vol. PER-7, no. 7, pp. 66-67, July 1987.

[55] E. Lunney, M. Ban, N. Duic, and A. Foley, "A state-ofthe-art review and feasibility analysis of high altitude wind 
power in northern ireland," Renewable and Sustainable Energy Reviews, vol. 68, pp. 899 - 911, 2017. [Online]. Available: http: //www.sciencedirect.com/science/article/pii/S1364032116304282

[56] P. Sarajcev, "Assessment of lightning stroke incidence to modern wind turbines," in SoftCOM 2010, 18th International Conference on Software, Telecommunications and Computer Networks, Sep. 2010, pp. 97-101.

[57] D. Djalel, M. Mourad, and G. Sihem, "Contribution to the study of the aggression of lightning phenomenon on the wind turbine structures," Wind Engineering, vol. 40, no. 1, pp. 100-111, 2016.

[58] P. Sarajcev, J. Vasilj, and D. Jakus, "Monte-carlo analysis of wind farm lightning-surge transients aided by linet lightningdetection network data," Renewable Energy, vol. 99, pp. 501 - 513, 2016. [Online]. Available: http://www.sciencedirect.com/science/ article/pii/S0960148116306085

[59] A. Candela Garolera, K. L. Cummins, S. F. Madsen, J. Holboell, and J. D. Myers, "Multiple lightning discharges in wind turbines associated with nearby cloud-to-ground lightning," IEEE Transactions on Sustainable Energy, vol. 6, no. 2, pp. 526-533, April 2015.

[60] V. Peesapati and I. Cotton, "Lightning protection of wind turbines a comparison of real lightning strike data and finite element lightning attachment analysis," in 2009 International Conference on Sustainable Power Generation and Supply, April 2009, pp. 1-8.

[61] V. Peesapati, I. Cotton, T. Sorensen, T. Krogh, and N. Kokkinos, "Lightning protection of wind turbines - a comparison of measured data with required protection levels," IET Renewable Power Generation, vol. 5, no. 1, pp. 48-57, January 2011. 
[62] S. F. Madsen, A. C. Garolera, K. Bertelsen, and H. I. Park, "Lightning Protection Zoning and Risk Exposure Assessment of Wind Turbines," EWEA Offshore, pp. 1-9, 2015.

[63] International Electrotechnical Commission, "IEC 62305 Protection Against Lightning-Part 1: General Principles," 2010.

[64] H. Heidler, "Analytische blitzstromfunktion zur lemp-berechnung," 18th ICLP, Munich, Germany, 1985, 1985.

[65] F. Heidler, J. M. Cvetic, and B. V. Stanic, "Calculation of lightning current parameters," IEEE Transactions on Power Delivery, vol. 14, no. 2, pp. 399-404, April 1999.

[66] A. De Conti and S. Visacro, "Analytical representation of single- and double-peaked lightning current waveforms," IEEE Transactions on Electromagnetic Compatibility, vol. 49, no. 2, pp. 448-451, May 2007.

[67] J. Bermudez, C. Pena, F. Rachidi, F. Heidler, and V. Rakov, "Analytical representation of lightning current waveforms using genetic algorithms," in International Conference on Lightning Protection, ICLP, 2004.

[68] K. Elrodesly, "Comparison between heidler function and the pulse function for modeling the lightning return-stroke current," Ph.D. dissertation, Ryerson University, 2010.

[69] K. Bitner, "Modeling of the lightning return stroke current at a tall structure using the derivative of the heidler function," MASc Thesis, Ryerson University, Toronto, 2004.

[70] F. Heidler and J. Cvetić, "A class of analytical functions to study the lightning effects associated with the current front," European transactions on electrical power, vol. 12, no. 2, pp. 141-150, 2002. 
[71] C. A. Nucci, G. Diendorfer, M. A. Uman, F. Rachidi, M. Ianoz, and C. Mazzetti, "Lightning return stroke current models with specified channel-base current: A review and comparison," Journal of Geophysical Research: Atmospheres, vol. 95, no. D12, pp. 20395-20 408, 1990.

[72] A. Karwowski and A. Zeddam, “Transient currents on lightning protection systems due to the indirect lightning effect," IEE Proceedings - Science, Measurement and Technology, vol. 142, no. 3, pp. 213222, May 1995.

[73] D. Rajičić and M. Todorovski, "Two-component current waveform for lightning simulation," IEEE Transactions on Electromagnetic Compatibility, vol. 57, no. 5, pp. 1062-1069, Oct 2015.

[74] A. Smorgonskiy, F. Rachidi, M. Rubinstein, N. V. Korovkin, and A. P. Vassilopoulos, "Are standardized lightning current waveforms suitable for aircraft and wind turbine blades made of composite materials?" IEEE Transactions on Electromagnetic Compatibility, vol. 59, no. 4, pp. 1320-1328, Aug 2017.

[75] P. Chowdhuri, J. G. Anderson, W. A. Chisholm, T. E. Field, M. Ishii, J. A. Martinez, M. B. Marz, J. McDaniel, T. E. McDermott, A. M. Mousa, T. Narita, D. K. Nichols, and T. A. Short, "Parameters of lightning strokes: a review," IEEE Transactions on Power Delivery, vol. 20, no. 1, pp. 346-358, Jan 2005.

[76] Y. Yasuda, N. Uno, H. Kobayashi, and T. Funabashi, "Surge analysis on wind farm when winter lightning strikes," IEEE Transactions on Energy Conversion, vol. 23, no. 1, pp. 257-262, March 2008.

[77] Y. Wang and W. Hu, "Investigation of the effects of receptors on the lightning strike protection of wind turbine blades," IEEE Transactions on Electromagnetic Compatibility, vol. 59, no. 4, pp. 1180-1187, Aug 2017. 
[78] A. S. Bretas, R. J. Cabral, R. C. Leborgne, G. D. Ferreira, and J. A. Morales, "Multi-objective MILP model for distribution systems reliability optimization: A lightning protection system design approach," International Journal of Electrical Power E Energy Systems, vol. 98, pp. 256-268, 2018.

[79] S. Pastromas, K. Sandros, K. Koutras, and E. Pyrgioti, "Investigation of lightning strike effects on wind turbine critical components," in 2018 IEEE International Conference on High Voltage Engineering and Application (ICHVE). IEEE, 2018, pp. 1-4.

[80] B. Glushakow, "Effective lightning protection for wind turbine generators," IEEE Transactions on Energy Conversion, vol. 22, no. 1, pp. 214-222, March 2007.

[81] J. Montanyà, O. van der Velde, and E. R. Williams, "Lightning discharges produced by wind turbines," Journal of Geophysical Research: Atmospheres, vol. 119, no. 3, pp. 1455-1462. [Online]. Available: https://agupubs.onlinelibrary.wiley.com/doi/abs/10. 1002/2013JD020225

[82] K. Chandrasekaran and G. S. Punekar, "Use of genetic algorithm to determine lightning channel-base current-function parameters," IEEE Transactions on Electromagnetic Compatibility, vol. 56, no. 1, pp. 235-238, Feb 2014.

[83] F. Fuchs, E. U. Landers, R. Schmid, and J. Wiesinger, "Lightning current and magnetic field parameters caused by lightning strikes to tall structures relating to interference of electronic systems," IEEE Transactions on Electromagnetic Compatibility, vol. 40, no. 4, pp. 444-451, Nov 1998.

[84] R. Rodrigues, V. Mendes, and J. Catalão, “Estimation of lightning vulnerability points on wind power plants using the rolling sphere 
method," Journal of Electrostatics, vol. 67, no. 5, pp. $774-780$, 2009. [Online]. Available: http://www.sciencedirect.com/science/ article/pii/S0304388609001417

[85] J. L. Jiang, H. C. Chang, C. C. Kuo, and C. K. Huang, "Transient overvoltage phenomena on the control system of wind turbines due to lightning strike," Renewable Energy, vol. 57, pp. 181 - 189, 2013. [Online]. Available: http://www.sciencedirect.com/science/ article/pii/S0960148113000451

[86] A. Candela Garolera, S. F. Madsen, M. Nissim, J. D. Myers, and J. Holboell, "Lightning damage to wind turbine blades from wind farms in the u.s." IEEE Transactions on Power Delivery, vol. 31, no. 3, pp. 1043-1049, June 2016.

[87] N. Malcolm and R. K. Aggarwal, "The impact of multiple lightning strokes on the energy absorbed by mov surge arresters in wind farms during direct lightning strikes," Renewable Energy, vol. 83, pp. 1305 - 1314, 2015. [Online]. Available: http: / /www.sciencedirect.com/science/article/pii/S0960148115003717

[88] International Electrotechnical Commission, "IEC 62305 Protection Against Lightning-Part 3: Physical Damage to Structures and Life Hazard," 2010.

[89] N. Ahmad, M. Ab-Kadir, M. Izadi, N. Azis, M. Radzi, N. Zaini, and M. Nasir, "Lightning protection on photovoltaic systems: A review on current and recommended practices," Renewable and Sustainable Energy Reviews, vol. 82, pp. 1611 - 1619, 2018. [Online]. Available: http: / /www.sciencedirect.com/science/article/pii/S1364032117310705

[90] DEHN - Lightning protection guide. [Online]. Available: http://www.ipdgroup.com.au/PDF/catalogues_and_datasheets / 
Brochures/10_Surge\%20Protection/35_DS702_E_2014_Lightning_ Protection_Guide.pdf

[91] R. Zoro and A. Purwadi, "The use of wind turbine structure for lightning protection system," in Proceedings of the 2011 International Conference on Electrical Engineering and Informatics, July 2011, pp. 1-6.

[92] A. J. Surtees, "A review of IEC 62305-4 protection against lightning part 4: Electrical and electronic systems within structures," in 2011 7th Asia-Pacific International Conference on Lightning, Nov 2011, pp. $478-481$.

[93] G. Parise, L. Martirano, and M. Lucheroni, "Level, class, and prospected safety performance of a lightning protection system for a complex of structures (lpcs)," IEEE Transactions on Industry Applications, vol. 46, no. 5, pp. 2106-2110, Sep. 2010.

[94] R. W. Hotchkiss, “Nema 5VS survey results on surge protective devices for the protection of safety equipment," in 2014 IEEE PES General Meeting I Conference Exposition, July 2014, pp. 1-4.

[95] S. Roberto Santos, "Surge protection for explosion hazard areas: Principles for ex-proof device," in 2013 International Symposium on Lightning Protection (XII SIPDA), Oct 2013, pp. 365-368.

[96] T. Kisielewicz, G. B. L. Piparo, C. Mazzetti, and A. Rousseau, “Dimensioning of SPD for the protection against surges due to lightning to lv overhead lines," in 2014 International Conference on Lightning Protection (ICLP), Oct 2014, pp. 141-145.

[97] C. Qinghua, Y. Lixia, and Y. Shu, "Ungrounded lightning surge protection device for wireless sensor networks node in the wilderness," in 2018 Cross Strait Quad-Regional Radio Science and Wireless Technology Conference (CSQRWC), July 2018, pp. 1-3. 
[98] V. March, "Key issues to define a method of lightning risk assessment for wind farms," Electric Power Systems Research, vol. 159, pp. 50 - 57, 2018, recent Developments on Lightning Research and Protection Technologies. [Online]. Available: http: / / www.sciencedirect.com/science/article/pii/S0378779617303450

[99] International Electrotechnical Commission, "IEC 62305 Protection Against Lightning-Part 2: Risk Management," 2010.

[100] Lightning and surge protection of wind turbines. https://www.dehnusa.com/sites/default/files/uploads/dehn/pdf/whitepapers/ab-juli15/wp016-e-wind_turbines.pdf.

[101] K. Kajita, Y. Baba, N. Nagaoka, and A. Ametani, “Computation of lightning electromagnetic pulses using the constrained interpolation profile method," Electric Power Systems Research, vol. 115, pp. 94 - 101, 2014, special issue based on selected expanded contributions from the 10th International Conference on Power System Transients (IPST). [Online]. Available: http://www. sciencedirect.com/science/article/pii/S0378779614000625

[102] O. Skoblikov and V. Kniaziev, "Penetration of lightning electromagnetic pulses into metallic enclosures with apertures," Electric Power Systems Research, vol. 113, pp. 48 - 63, 2014, lightning Research and Lightning Protection Technologies. [Online]. Available: http: / / www.sciencedirect.com/science/article/pii/S0378779614001011

[103] International Electrotechnical Commission, "IEC 62305 Protection Against Lightning-Part 4: Electrical and Electronic Systems Within Structures," 2010.

[104] A. Tatematsu, F. Rachidi, and M. Rubinstein, "Calculation of electromagnetic fields inside a building with layered reinforcing bar struck 
by lightning using the fdtd method," in 2014 International Symposium on Electromagnetic Compatibility, Tokyo, May 2014, pp. 386-389.

[105] Y. Baba and V. A. Rakov, "Applications of the FDTD method to lightning electromagnetic pulse and surge simulations," IEEE Transactions on Electromagnetic Compatibility, vol. 56, no. 6, pp. 1506-1521, Dec 2014.

[106] Y. Baba and V. A. Rakov, "Applications of the FDTD method to lightning electromagnetic pulse and surge simulations," in 2014 International Conference on Lightning Protection (ICLP), Oct 2014, pp. 325-339.

[107] F. Grange, A. Sellier, S. Journet, and F. P. Dawalibi, “On the assessment of the voltage drop between two lightning protection zones connected by a signal line," in 2018 34th International Conference on Lightning Protection (ICLP), Sep. 2018, pp. 1-6.

[108] A. Orlandi and F. Schietroma, "Attenuation by a lightning protection system of induced voltages due to direct strikes to a building," IEEE Transactions on Electromagnetic Compatibility, vol. 38, no. 1, pp. 43-50, Feb 1996.

[109] I. A. Metwally and F. H. Heidler, "Reduction of lightning-induced magnetic fields and voltages inside struck double-layer grid-like shields," IEEE Transactions on Electromagnetic Compatibility, vol. 50, no. 4, pp. 905-912, Nov 2008.

[110] T. Maksimowicz and K. Aniserowicz, "Investigation of models of grid-like shields subjected to lightning electromagnetic field: Experiments in the frequency domain," IEEE Transactions on Electromagnetic Compatibility, vol. 54, no. 4, pp. 826-836, Aug 2012.

[111] P. Sarajcev, D. Jakus, J. Vasilj, and S. Vodopija, "Application of genetic algorithm in designing high-voltage open-air substation lightning protection system," Journal of Electrostatics, vol. 93, pp. 
43 - 51, 2018. [Online]. Available: http:/ /www.sciencedirect.com/ science/article/pii/S0304388617302930

[112] S. Sekioka, "Chapter 8 - Lightning protections of renewable energy generation systems," in Integration of Distributed Energy Resources in Power Systems, T. Funabashi, Ed. Academic Press, 2016, pp. 193 - 228. [Online]. Available: http:/ / www.sciencedirect.com/science/ article/pii/B9780128032121000088

[113] Z. Flisowski and P. Sul, "Subtleties affecting the efficiency of the structure lightning and overvoltage protection," in 2018 34th International Conference on Lightning Protection (ICLP), Sep. 2018, pp. 1-4.

[114] S. Fisahn, S. Koj, and H. Garbe, "Modelling of multi-megawatt wind turbines for emi and ems investigations by a topological approach," in 2017 XXXIInd General Assembly and Scientific Symposium of the International Union of Radio Science (URSI GASS), Aug 2017, pp. 1-4.

[115] S. F. Madsen, K. Bertelsen, T. H. Krogh, H. V. Erichsen, A. N. Hansen, and K. B. Lønbæk, "Proposal of new zoning concept considering lightning protection of wind turbine blades," in 2010 30th International Conference on Lightning Protection (ICLP), Sep. 2010, pp. 1-7.

[116] F. L. Pironnec and J. Aspas-Puértolas, "Electrostatic field and lightning zoning analysis of a windmill: Study of current and innovative protection strategies," in 2014 International Conference on Lightning Protection (ICLP), Oct 2014, pp. 659-666.

[117] N. McDonagh and D. Klopotan, "The development of a 3-D rolling sphere algorithm for lightning protection," in 2012 47th International Universities Power Engineering Conference (UPEC), Sep. 2012, pp. 1-5.

[118] T. Horvath, "Construction of the protected volume with proper application of rolling sphere method," in 2010 30th International Conference on Lightning Protection (ICLP), Sep. 2010, pp. 1-5. 
[119] Q. Xie, S. Baron, S. Fortin, and F. P. Dawalibi, "Rolling sphere method using 3D graphics approach," in 2009 Asia-Pacific Power and Energy Engineering Conference, March 2009, pp. 1-4.

[120] Y. Méndez, J. Birkl, S. Madsen, T. Sørensen, J. Plumer, and J. Montanya, "The 2018 revision of the standard IEC 61400-24: Lightning protection of wind turbines," in 2018 34th International Conference on Lightning Protection (ICLP), Sep. 2018, pp. 1-6.

[121] T. S. Sorensen, J. Plumer, J. Montanyà, T. H. Krogh, B. Hermoso, J. Birkl, T. Gehlhaar, B. McNiff, K. Bertelsen, V. Peesapati et al., "The update of IEC 61400-24 lightning protection of wind turbines," in 29th International Conference on Lightning Protection of Wind Turbines, 2008.

[122] A. Candela Garolera, "Lightning protection of flap system for wind turbine blades," Ph.D. dissertation, 2014.

[123] R. B. Rodrigues, V. M. F. Mendes, and J. P. S. Catalao, “Computer simulation for lightning and overvoltage protection of wind power plants," in 2008 4th IET Conference on Power Electronics, Machines and Drives, April 2008, pp. 777-782.

[124] C. F. Mieritz, A. C. Garolera et al., "Numerical tools for lightning protection of wind turbines," in 2013 International Conference on Lightning and Static Electricity, 2013.

[125] M. R. Ahmed and U. K. Das, “Lightning surge response of wind turbine grounding at low resistivity soil," in 8th International Conference on Electrical and Computer Engineering, Dec 2014, pp. 599-602.

[126] K. Yamamoto, Y. Kubo, and S. Sumi, "Transient grounding characteristic of wind turbines affecting back-flow lightning current into distribution system," in 2016 33rd International Conference on Lightning Protection (ICLP), Sep. 2016, pp. 1-5. 
[127] H. K. Karegar and A. Mousavi, "Wind turbines grounding system, boundary element approach," in 2008 Joint International Conference on Power System Technology and IEEE Power India Conference, Oct 2008, pp. 1-4.

[128] K. Yamamoto, S. Yanagawa, S. Sekioka, and S. Yokoyama, "Transient grounding characteristics of an actual wind turbine generator system at a low-resistivity site," IEEJ Transactions on Electrical and Electronic Engineering, vol. 5, no. 1, pp. 21-26, 2010.

[129] N. K. Gouvalas, I. F. Gonos, and I. A. Stathopulos, "Impact study of short-circuit calculation methods on the design of a wind farm's grounding system," Renewable Energy, vol. 66, pp. 25 - 32, 2014. [Online]. Available: http://www.sciencedirect.com/science/ article/pii/S0960148113006472

[130] M. Lorentzou, I. Cotton, N. Hatziargyriou, and N. Jenkins, “Electromagnetic analysis of wind turbine grounding systems," in Proc. Eur. Wind Energy Conf., 1997.

[131] A. C. Y. M. Hernández, G. Drobnjak, and M. Kizilcay, “An engineering approach in modeling lightning effects on megawatt-class onshore wind turbines using emtp and models," Progress In Electromagnetics Research, vol. 1309, 2011.

[132] Ö. Gül, "Analysis of wind turbine grounding systems in terms of touch and step voltages," Istanbul University-Journal of Electrical $\mathcal{E}$ Electronics Engineering, vol. 15, no. 1, pp. 1867-1872, 2015.

[133] M. I. Lorentzou, N. D. Hatziargyriou, and B. C. Papadias, "Analysis of wind turbine grounding systems," in 2000 10th Mediterranean Electrotechnical Conference. Information Technology and Electrotechnology for the Mediterranean Countries. Proceedings. MeleCon 2000 (Cat. No.00CH37099), vol. 3, May 2000, pp. 936-939 vol.3. 
[134] M. Abd-Allah, M. N. Ali, and A. Said, "A proper design of wind turbine grounding systems under lightning," World Academy of Science, Engineering and Technology, International Science Index 94, International Journal of Electrical, Robotics, Electronics and Communications Engineering, vol. 8, no. 10, pp. 1358-1365, 2014.

[135] G. P. Kondylis, K. D. Damianaki, V. P. Androvitsaneas, and I. F. Gonos, "Simplified formulae method for estimating wind turbine generators ground resistance," IEEE Transactions on Power Delivery, vol. 33, no. 6, pp. 2829-2836, Dec 2018.

[136] M. Talaat, M. Farahat, and M. Osman, "Assessment of earthing system location for wind turbines using finite element method," Renewable Energy, vol. 93, pp. 412-423, 2016.

[137] R. Hoerauf, "Considerations in wind farm grounding designs," IEEE Transactions on Industry Applications, vol. 50, no. 2, pp. 13481355, March 2014.

[138] H. K. Karegar and M. Arabi, "New wind turbine grounding system to reduce step amp; touch voltage," in 2010 IEEE International Conference on Power and Energy, Nov 2010, pp. 168-171.

[139] S. A. Pastromas, K. Maimaris, I. K. Stasinos, I. A. Naxakis, and E. C. Pyrgioti, "Assessment of wind turbine grounding system," in 2018 IEEE International Conference on High Voltage Engineering and Application (ICHVE), Sep. 2018, pp. 1-4.

[140] I. Cotton, "Windfarm earthing," in 1999 Eleventh International Symposium on High Voltage Engineering, vol. 2, 1999, pp. 288-291 vol.2.

[141] J. Liu, R. D. Southey, and F. P. Dawalibi, "Application of advanced grounding design techniques to plant grounding systems," in 2005 IEEE/PES Transmission Distribution Conference Exposition: Asia and Pacific, Aug 2005, pp. 1-6. 
[142] N. Hatziargvriou, M. Lorentzou, and N. Jenkins, "Wind farm earthing," in IEE Half-Day Colloquium on Lightning Protection of Wind Turbines (Digest No: 1997/303),, Nov 1997, pp. 6/1-6/3.

[143] M. Ahmeda, "Earthing performance of transmission line towers," Ph.D. dissertation, Cardiff University, 2012.

[144] M. R. Ahmed and M. Ishii, "Electromagnetic analysis of lightning surge response of interconnected wind turbine grounding system," in 2011 International Symposium on Lightning Protection, Oct 2011, pp. 226-231.

[145] S. Visacro and R. Alipio, "Frequency dependence of soil parameters: Experimental results, predicting formula and influence on the lightning response of grounding electrodes," IEEE Transactions on Power Delivery, vol. 27, no. 2, pp. 927-935, April 2012.

[146] E. D. Sunde, Earth Conduction Effects in Transmission Systems. Dover Publications Inc., 1968.

[147] S. J. Schwarz, "Analytical expressions for the resistance of grounding systems [includes discussion]," Transactions of the American Institute of Electrical Engineers. Part III: Power Apparatus and Systems, vol. 73, no. 2, pp. 1011-1016, Jan 1954.

[148] Gilbert, Gary, "High voltage grounding systems," Ph.D. dissertation, 2011. [Online]. Available: http://hdl.handle.net/10012/5789

[149] W. Shan, Y. Liu, Z. Hu, and J. Xiao, "A model for the electrical resistivity of frozen soils and an experimental verification of the model," Cold Regions Science and Technology, vol. 119, pp. 75 - 83, 2015. [Online]. Available: http://www.sciencedirect.com/science/ article/pii/S0165232X15001718 
[150] J. Liu and F. P. Dawalibi, "Wind turbine farm network grounding design using integrated simulation methods and techniques," in 2010 International Conference on Future Power and Energy Engineering, June 2010, pp. 99-102.

[151] G. Gilbert, "Soil modelling techniques," International Journal of Materials Science and Applications, vol. 1, no. 1, pp. 8-13, 2012.

[152] Current Distribution, Electromagnetic Fields, Grounding and Soil Structure Analysis (CDEGS) software package. [Online]. Available: http://www.sestech.com/Products/SoftPackages/CDEGS.htm.

[153] W. CIGRE, "Guideline for numerical electromagnetic analysis method and its application to surge phenomena," CIGRE Brochure, vol. 543, 2013.

[154] C. Lee, C. Chang, and J. Jiang, "Evaluation of ground potential rises in a commercial building during a direct lightning stroke using cdegs," IEEE Transactions on Industry Applications, vol. 51, no. 6, pp. 4882-4888, Nov 2015.

[155] K. Yamamoto, S. Yanagawa, K. Yamabuki, S. Sekioka, and S. Yokoyama, "Analytical surveys of transient and frequencydependent grounding characteristics of a wind turbine generator system on the basis of field tests," IEEE Transactions on Power Delivery, vol. 25, no. 4, pp. 3035-3043, 2010.

[156] V. T. Kontargyri, I. F. Gonos, and I. A. Stathopulos, "Study on wind farm grounding system," IEEE Transactions on Industry Applications, vol. 51, no. 6, pp. 4969-4977, Nov 2015.

[157] B. Markovski, L. Grcev, and V. Arnautovski-Toseva, “Transient characteristics of wind turbine grounding," in 2012 International Conference on Lightning Protection (ICLP), Sep. 2012, pp. 1-6. 
[158] R. D. Southey, M. Siahrang, S. Fortin, and F. P. Dawalibi, "Using fall-of-potential measurements to improve deep soil resistivity estimates," IEEE Transactions on Industry Applications, vol. 51, no. 6, pp. 5023-5029, Nov 2015.

[159] P. J. Lagace, M. H. Vuong, M. Lefebvre, and J. Fortin, “Multilayer resistivity interpretation and error estimation using electrostatic images," IEEE Transactions on Power Delivery, vol. 21, no. 4, pp. 19541960, Oct 2006.

[160] J. Ma and F. P. Dawlibi, "Analysis of grounding systems in soils with cylindrical soil volumes," IEEE Transactions on Power Delivery, vol. 15, no. 3, pp. 913-918, July 2000.

[161] Jinxi Ma and F. P. Dawalibi, "Analysis of grounding systems in soils with finite volumes of different resistivities," IEEE Transactions on Power Delivery, vol. 17, no. 2, pp. 596-602, April 2002.

[162] S. Visacro, "A comprehensive approach to the grounding response to lightning currents," IEEE Transactions on power delivery, vol. 22, no. 1, pp. 381-386, 2007.

[163] R. Alipio and S. Visacro, "Frequency dependence of soil parameters: Effect on the lightning response of grounding electrodes," IEEE Transactions on Electromagnetic Compatibility, vol. 55, no. 1, pp. 132139, Feb 2013.

[164] M. Zhou, J. Wang, L. Cai, Y. Fan, and Z. Zheng, "Laboratory investigations on factors affecting soil electrical resistivity and the measurement," IEEE Transactions on Industry Applications, vol. 51, no. 6, pp. 5358-5365, Nov 2015.

[165] G. C. Topp, J. L. Davis, and A. P. Annan, “Electromagnetic determination of soil water content: Measurements in coaxial transmission lines," Water Resources Research, vol. 16, no. 3, pp. 
574-582. [Online]. Available: https://agupubs.onlinelibrary.wiley. com/doi/abs/10.1029/WR016i003p00574

[166] R. Porretta and F. Bianchi, "Profiles of relative permittivity and electrical conductivity from unsaturated soil water content models," Annals of Geophysics, vol. 59, no. 3, p. 0320, 2016.

[167] "IEEE guide for safety in ac substation grounding," IEEE Std 802013 (Revision of IEEE Std 80-2000/ Incorporates IEEE Std 80-2013/Cor 1-2015), pp. 1-226, May 2015.

[168] Y. Yasuda and T. Fujii, "Equivalent equation of earth resistance for ring electrode of wind turbine," in 2010 30th International Conference on Lightning Protection (ICLP), Sept 2010, pp. 1-4.

[169] D. Cavka, D. Poljak, V. Doric, and R. Goic, "Transient analysis of grounding systems for wind turbines," Renewable Energy, vol. 43, pp. 284 - 291, 2012. [Online]. Available: http://www.sciencedirect. com/science/article/pii/S0960148111006446

[170] P. Boys, "Resistivity testing for earthing safety," in 2016 Down to Earth Conference (DTEC), Sep. 2016, pp. 1-10.

[171] E. Mombello, O. Trad, J. Rivera, and A. Andreoni, "Two-layer soil model for power station grounding system calculation considering multilayer soil stratification," Electric Power Systems Research, vol. 37, no. 1, pp. 67 - 78, 1996. [Online]. Available: http: //www.sciencedirect.com/science/article/pii/0378779696010425

[172] F. P. Dawalibi, J. Ma, and R. D. Southey, "Behaviour of grounding systems in multilayer soils: a parametric analysis," IEEE Transactions on Power Delivery, vol. 9, no. 1, pp. 334-342, Jan 1994.

[173] "IEEE guide for measuring earth resistivity, ground impedance, and earth surface potentials of a grounding system," IEEE Std 81-2012 (Revision of IEEE Std 81-1983), pp. 1-86, Dec 2012. 
[174] A. Sunjerga, Q. Li, D. Poljak, M. Rubinstein, and F. Rachidi, “Transient impedance of interconnected wind turbine grounding systems," in 2018 26th International Conference on Software, Telecommunications and Computer Networks (SoftCOM). IEEE, 2018, pp. 1-4.

[175] R. Alipio, M. T. C. de Barros, M. A. O. Schroeder, and K. Yamamoto, "Lightning and low-frequency performance of interconnected grounding systems of wind turbines," in 2018 34th International Conference on Lightning Protection (ICLP), Sep. 2018, pp. 1-6.

[176] Current Distribution, Electromagnetic Fields, Grounding and Soil Structure Analysis (CDEGS) software validation. [Online]. Available: https: / /www.sestech.com/en/Product/SoftwareValidation.

[177] A. Kyritsis, D. Voglitsis, N. Papanikolaou, S. Tselepis, C. Christodoulou, I. Gonos, and S. Kalogirou, "Evolution of PV systems in greece and review of applicable solutions for higher penetration levels," Renewable Energy, vol. 109, pp. 487 - 499, 2017. [Online]. Available: http: / / www.sciencedirect.com/science/article/pii/S0960148117302574

[178] R. D. Goud, R. Rayudu, C. P. Moore, and D. Burmester, "Lightning protection analysis of main shaft bearings in wind turbine generators," in 2016 IEEE International Conference on Power System Technology (POWERCON), Sep. 2016, pp. 1-6.

[179] A. Šunjerga, D. S. Gazzana, D. Poljak, H. Karami, K. Sheshyekani, M. Rubinstein, and F. Rachidi, "Tower and path-dependent voltage effects on the measurement of grounding impedance for lightning studies," IEEE Transactions on Electromagnetic Compatibility, vol. 61, no. 2, pp. 409-418, April 2019. 
[180] S. Sekioka, H. Otoguro, and T. Funabashi, "A study on overvoltages in windfarm caused by direct lightning stroke," IEEE Transactions on Power Delivery, vol. 34, no. 2, pp. 671-679, April 2019.

[181] N. Harid, H. Griffiths, S. Mousa, D. Clark, S. Robson, and A. Haddad, "On the analysis of impulse test results on grounding systems," IEEE Transactions on Industry Applications, vol. 51, no. 6, pp. 53245334, Nov 2015.

[182] K. Yamamoto, S. Sumi, S. Sekioka, and J. He, “Derivations of effective length formula of vertical grounding rods and horizontal grounding electrodes based on physical phenomena of lightning surge propagations," IEEE Transactions on Industry Applications, vol. 51, no. 6, pp. 4934-4942, Nov 2015.

[183] J. He and B. Zhang, "Progress in lightning impulse characteristics of grounding electrodes with soil ionization," IEEE Transactions on Industry Applications, vol. 51, no. 6, pp. 4924-4933, Nov 2015.

[184] A. Ackerman, P. K. Sen, and C. Oertli, “Designing safe and reliable grounding in ac substations with poor soil resistivity: An interpretation of ieee std. 80," IEEE Transactions on Industry Applications, vol. 49, no. 4, pp. 1883-1889, July 2013.

[185] K. Yamamoto, T. Ookawa, and S. Sumi, "Study of the spread of potential rise between two grounding electrodes," IEEE Transactions on Industry Applications, vol. 51, no. 6, pp. 5247-5253, Nov 2015.

[186] S. Visacro, "What engineers in industry should know about the response of grounding electrodes subject to lightning currents," IEEE Transactions on Industry Applications, vol. 51, no. 6, pp. 4943-4951, Nov 2015.

[187] L. Grcev, "Impulse efficiency of ground electrodes," IEEE Transactions on Power Delivery, vol. 24, no. 1, pp. 441-451, Jan 2009. 
[188] F. Freschi, M. Mitolo, and M. Tartaglia, "Interferences phenomena between separate grounding systems," IEEE Transactions on Industry Applications, vol. 50, no. 4, pp. 2853-2860, July 2014.

[189] B. Markovski, L. Grcev, and V. Arnautovski-Toseva, "Step and touch voltages near wind turbine grounding during lightning strokes," in International Symposium on Electromagnetic Compatibility - EMC EUROPE, Sept 2012, pp. 1-6.

[190] G. Parise, L. Martirano, L. Parise, S. Celozzi, and R. Araneo, "Simplified conservative testing method of touch and step voltages by multiple auxiliary electrodes at reduced distance," IEEE Transactions on Industry Applications, vol. 51, no. 6, pp. 4987-4993, Nov 2015.

[191] A. Kern, R. Brocke, V. Raab, M. Hannig, M. Rock, O. Beierl, and W. Zischank, "Detailed calculation of interception efficiencies for air-termination systems using the dynamic electro-geometrical model - practical applications," in 2016 33rd International Conference on Lightning Protection (ICLP), Sep. 2016, pp. 1-6.

[192] General specification - V112-3.0 MW 50/60 Hz. [Online]. Available: https://stopthesethings.files.wordpress.com/2015/12/ vestas-v112-specs.pdf

[193] S. Kazazi and K. Raahemifar, "Genetic algorithm technique on wind turbine and sensitive equipment placement against lightning," in 2013 26th IEEE Canadian Conference on Electrical and Computer Engineering (CCECE), May 2013, pp. 1-6.

[194] M. Mitolo, P. E. Sutherland, and R. Natarajan, "Effects of high fault currents on ground grid design," IEEE Transactions on Industry Applications, vol. 46, no. 3, pp. 1118-1124, May 2010.

[195] R. Alipio, M. A. O. Schroeder, and M. M. Afonso, "Voltage distribution along earth grounding grids subjected to lightning currents," 
IEEE Transactions on Industry Applications, vol. 51, no. 6, pp. 49124916, Nov 2015.

[196] A. Yamanaka, N. Nagaoka, and H. Morita, "Effect of inductive grounding impedance on lightning transients in wind farm," in 2018 34th International Conference on Lightning Protection (ICLP), Sep. 2018, pp. 1-6.

[197] W. P. Calixto, L. M. Neto, M. Wu, K. Yamanaka, and E. da Paz Moreira, "Parameters estimation of a horizontal multilayer soil using genetic algorithm," IEEE Transactions on Power Delivery, vol. 25, no. 3, pp. 1250-1257, 2010.

[198] I. F. Gonos and I. A. Stathopulos, "Estimation of multilayer soil parameters using genetic algorithms," IEEE Transactions on Power Delivery, vol. 20, no. 1, pp. 100-106, Jan 2005.

[199] D. A. Tsiamitros, G. K. Papagiannis, and P. S. Dokopoulos, "Homogenous earth approximation of two-layer earth structures: An equivalent resistivity approach," IEEE transactions on power delivery, vol. 22, no. 1, pp. 658-666, 2007.

[200] Y. Yasuda, T. Fujii, and T. Ueda, "How does ring earth electrode effect to wind turbine?" in 2007 42nd International Universities Power Engineering Conference, Sept 2007, pp. 796-799. 